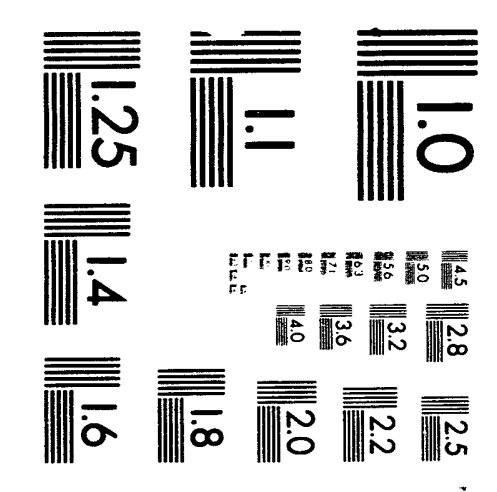



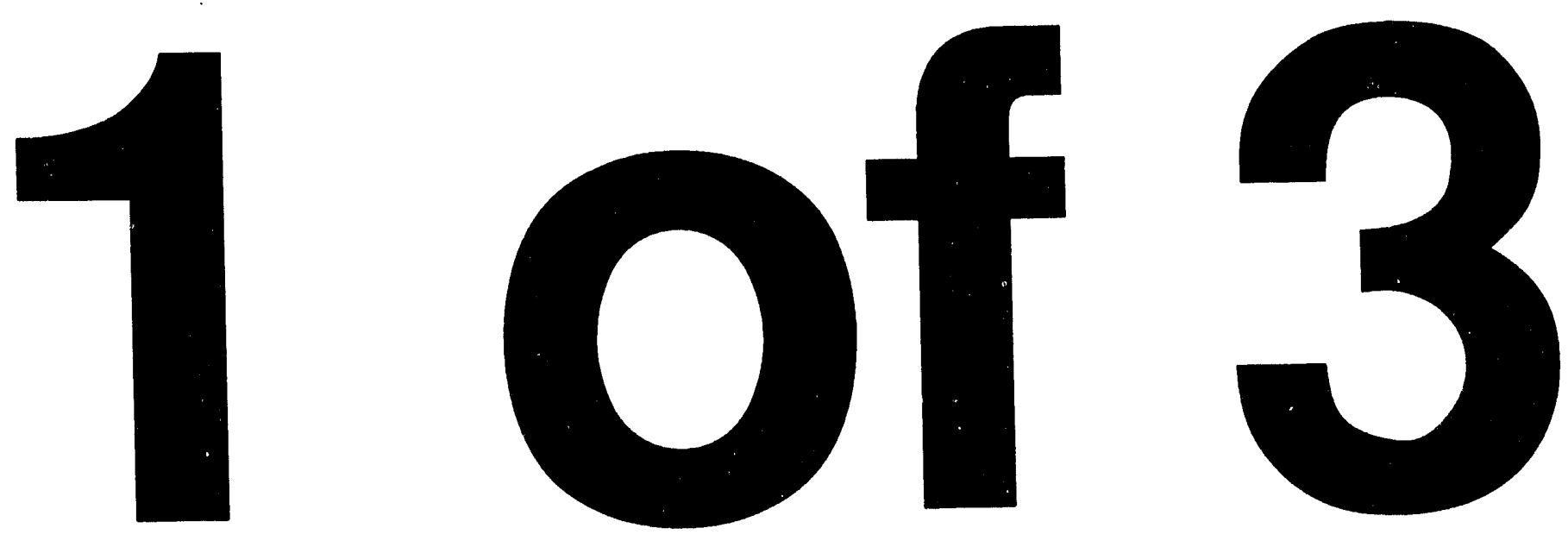
Scalar Operators in Solid-State NMR

by

Boqin Sun

Department of Chemistry

University of California

and

Materials Sciences Division

Lawrence Berkeley Laboratory

University of California

Berkeley, California 94720

November 1991

This work was supported by the Director, Office of Energy Research, Office of Basic Energy Sciences, Materials Sciences Division of the U.S. Department of Energy, under Contract No. DE-AC03-76SF00098.

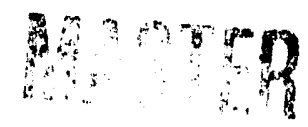

DisTMBUTION a: ras nocument is UWLIMITEB 


\title{
Scalar Operators in Solid-State NMR
}

\author{
Copyright (C)1991 \\ by \\ Boqin Sun
}

\begin{abstract}
The Goverment reserves for itself and others acting on its behalf a royalty free, nonexclusive, irrevocable, world-wide license for govermental purposes to publish, distribute, translate, duplicate, exhibit, and perform any such data copyrighted by the contractor.
\end{abstract}

The U.S. Department of Energy has the right to use this thesis for any purpose whatsoever including the right to reproduce all or any part thereof 


\title{
Scalar Operators in Solid-State NMR
}

by

Boqin Sun

\begin{abstract}
Selectivity and resolution of solid-state NMR spectra are determined by the dispersion of local magnetic fields originating from relaxation effects and orientationdependent resonant frequencies of spin nuclei. Theoretically, the orientation-dependent resonant frequencies can be represented by a set of irreducible tensors. Among these tensors, only zero rank tensors (scalar operators) are capable of providing high resolution NMR spectra. In this thesis, I present a series of new developments in high resolution solid-state NMR concerning the reconstruction of various scalar operators.

In chapter 1 a new method for calculating zero field NMR spectra is described, and the evolution matrices of the density matrix are evaluated. Zero field order selective observation, zero-high field correlation spectroscopy, and measurement of zero field spin diffusion are then introduced. Order selective observation can be used to simplify zero field spectra. Zero-high field correlation spectroscopy results in twodimensional spectra from which all information available in zero and high field spin interactions can be obtained. The spin diffusion rates in zero field are determined by the square of the dipolar coupling constants and the relative orientations between the dipolar and the quadrupolar interactions.

A general theory of zero field NMR in high field is described in chapter 2. A coupled space is introduced in which the high field spin Hamiltonian is expressed as irreducible tensors from rank zero to four. The scalar operator in this Hamiltonian can be extracted by dynamic angle spinning (DAS), double rotation (DOR), dynamic angle hopping (DAH), or pulse sequence for performing zero field NMR in high field. An analytical solution of these pulse sequence is found, and additional new trajectories are also presented.
\end{abstract}


Second order NMR spectroscopy of half integer quadrupolar spin nuclei is described in chapter 3. The first order average Hamiltonian is evaluated. The resulting powder lineshapes of the central transitions are simulated. The variations of these powder lineshapes and the their singularities upon the variable angle sample spinning (VASS) are discussed. The theoretical and experimental aspects of dynamic angle spinning (DAS) and double rotation (DOR) are also thoroughly described. Finally, a general method for averaging out arbitrary rank tensors appearing in Hamiltonian is developed using group theory arguments.

Chapter 4 presents calculations of MAS, DOR, and DAS sideband intensities. A new method of calculating MAS sideband intensities is discussed. These intensities can be calculated in real time, and allow CSA parameters to be easily extracted. DOR and DAS sidebands are analyzed by moment expansion and Bessel function analysis. The results show that DOR and DAS sideband intensities depend strongly on the relative rotor phase and the structure of both DOR and DAS sidebands is complicated due to the interference between spinning speeds (in DAS the time ratio can be converted to two spinning speeds).

The general theory of chemical exchange processes in solid-state NMR is treated in chapter 5. The lineshape changes during the chemical exchange are reviewed and discussed. The motion in icosahedral $\left(C_{60}\right)$ molecules is then analyzed. Lineshape changes due to different jump modes occur smoothly, similar to those observed in the strong collision limit. Comparing with the experimental results, there are indied different phases in the carbon clusters, and motions in $C_{60}$ have different correlation times within these different phases.

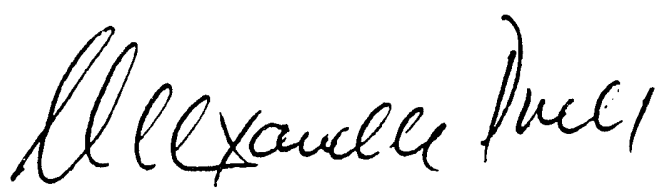




\section{Contents}

1 Zero Field NMR and NQR Spectroscopy 1

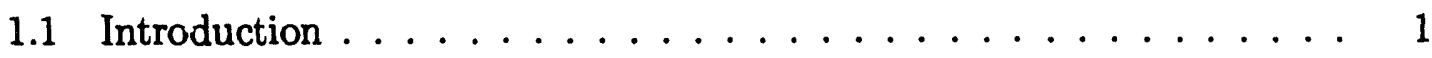

1.2 Calculation of Zero Field NMR and NQR Spectra . . . . . . . . . . 3

1.3 Order Selective Observation of Zero Field Signals . . . . . . . . . . 11

1.4 Zero Field and High Field Correlation Spectrum . . . . . . . . . . . 17

1.5 Spin Diffusion in Zero Field NMR . . . . . . . . . . . . . . 25

2 Theory of Zero Field NMR in High Field 42

2.1 Introduction . . . . . . . . . . . . . . . . 42

2.2 Reconstructing Scalar Hamiltonian in High Field . . . . . . . . . 44

2.3 Exact Solutions of Tycko's Pulse Sequence . . . . . . . . . . . . . 48

2.4 A New Sequence for Zero Field NMR in High Field . . . . . . . . . 56

2.5 Optimized Solutions . . . . . . . . . . . . . . . 58

2.5.1 Dynamic Angle Hopping (DAH) . . . . . . . . . 60

2.5.2 Dynamic Angle Spinning (DAS) for Zero Field NMR in High Field ...................... 62

2.5.3 Double Rotation (DOR) for Zero Field NMR in High Field . . 62

3 Second Order NMR Spectroscopy $\quad 66$

3.1 Introduction .................... 66

3.2 The First Order Average Hamiltonian with a Quadrupolar Interaction 67

3.3 Static Powder Lineshapes . . . . . . . . . . . . . . . . . 73 
3.4 Powder Lineshapes under VASS . . . . . . . . . . . . . 79

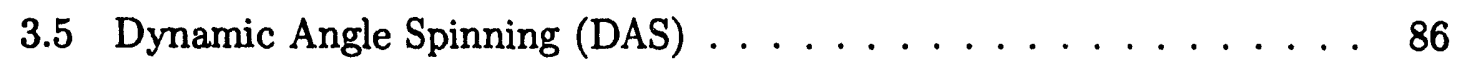

3.6 DAS Experiments . . . . . . . . . . . . . . . . . 97

3.7 Powder Lineshape in A Multi-Dimensional Space . . . . . . . . 108

3.8 Double Rotation (DOR) $\ldots \ldots \ldots \ldots \ldots \ldots$

3.9 DOR Experiments $\ldots \ldots \ldots \ldots \ldots \ldots \ldots \ldots \ldots$

3.10 Iterative Averaging of Tensorial Interactions $\ldots \ldots \ldots \ldots$

4 Calculation of Sideband Intensities in MAS, DAS, and DOR NMR145

4.1 An Approximate Method of MAS Sideband Intensity Calculation . . 145

4.1 .1 Introduction . . . . . . . . . . . . . . 145

4.1 .2 Theory . . . . . . . . . . . . . . . . . . 147

4.1 .3 Results . . . . . . . . . . . . . . . . . . 152

4.1 .4 Conclusions . . . . . . . . . . . . . . . . . 162

4.2 Sidebands in Double Rotation (DOR) NMR . . . . . . . . 163

4.2 .1 Introduction . . . . . . . . . . . . . 163

4.2 .2 Experimental Results . . . . . . . . . . . . . . . . 165

4.2.3 FID Signal under DOR . . . . . . . . . . . . . . . 165

4.2.4 Moment Analysis of DOR Sidebands . . . . . . . . 167

4.2.5 Bessel analysis of DOR sidebands . . . . . . . . . 171

4.2.6 Symmetry Properties of DOR Sidebands . . . . . . . 173

4.2 .7 Conclusions . . . . . . . . . . . . . . . . . . 181

4.3 Sidebands in DAS NMR Experiments . . . . . . . . . . . . 182

4.3.1 FID Signal of DAS NMR . . . . . . . . . . . . . 183

4.3.2 Moment Analysis of DAS FID signal . . . . . . . . . 184

4.3.3 Bessel Analysis of DAS Sidebands . . . . . . . . . . . 185

4.4 Conclusion . . . . . . . . . . . . . . . 190 
5 Motions in Solid $C_{60}$

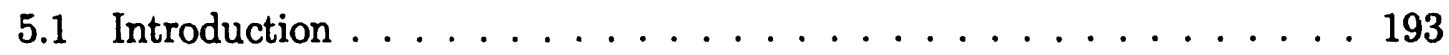

5.2 Theory of Chemical Exchange . . . . . . . . . . . . . . 194

5.3 Symmetry Properties of Discrete Jumps _. . . . . . . . . . . 204

5.4 Computational Approaches and Examples . . . . . . . . . 215

5.5 Jumps with An Icosahedral Symmetry . . . . . . . . . . . . 224

5.6 Motions in Solid $C_{60} \ldots \ldots \ldots \ldots \ldots \ldots$ 


\section{List of Figures}

1.1 Pulse scheme in zero field NMR . . . . . . . . . . . . 13

1.2 Response under the two pulse sequence in zero field NMR $\ldots \ldots 16$

1.3 Simulated powder pattern in $\mathrm{zf}$-hf correlation spectra . . . . . . . 21

1.4 Powder patterns of $\mathrm{zf}(\mathrm{D})$-hf(CSA) correlation spectra . . . . . . . 24

1.5 Pulse Scheme for zero field spin diffusion measurement . . . . . . 27

1.6 Experimental spin diffusion spectra of diethylterephthalate- $d_{4} \ldots 28$

1.7 Zero field deuterium spectrum of diethylterephthalate- $d_{4} \ldots \ldots$

1.8 Schematic energy levels for a two coupled spin system with $I=1$ in

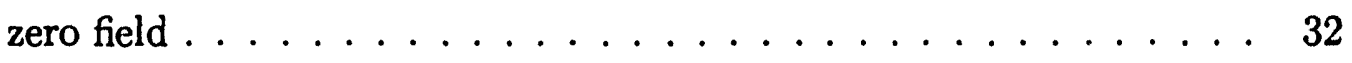

1.9 Schematic matrix representation of the dipolar Hamiltonian $\ldots . . .38$

2.1 Tycko's trajectory for zero field NMR in high field . . . . . . . . . 50

2.2 Exact solutions in Tycko's trajectory $\ldots \ldots \ldots \ldots \ldots$

2.3 Simulated spectra of zero field NMR in high field . . . . . . . 55

2.4 Exact solutions of a new trajectory $\ldots \ldots \ldots \ldots \ldots \ldots$

2.5 DAH trajectory $\ldots \ldots \ldots \ldots \ldots \ldots \ldots \ldots \ldots \ldots \ldots \ldots \ldots$

2.6 DAS trajectory $\ldots \ldots \ldots \ldots \ldots \ldots \ldots \ldots \ldots \ldots \ldots$

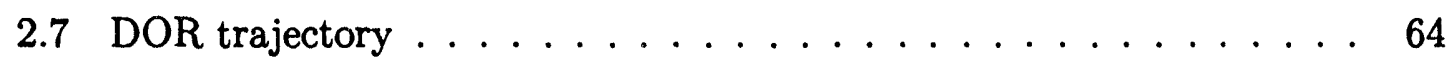

3.1 Variations of static second order powder patterns with $\eta \ldots \ldots 76$

3.2 Singularities observed in powder patterns with $\eta \ldots \ldots \ldots . \ldots 78$

3.3 Relationships between the laboratory frame, the SCF and the PAS . . 80 
3.4 VASS powder pattern $\ldots \ldots \ldots \ldots \ldots \ldots \ldots \ldots$

3.5 Boundary condition of the singularities $\ldots \ldots \ldots \ldots \ldots$

3.6 3D plot of the singularities . . . . . . . . . . . . . 84

3.7 Variations of Singularities with $\eta$ in VASS $\ldots \ldots \ldots . \ldots 85$

$3.8 \quad P_{2}$ and $P_{4}$ versus $\theta \ldots \ldots \ldots \ldots \ldots \ldots \ldots \ldots \ldots$

3.9 Second order powder pattern versus field strength $H_{0} \ldots \ldots \ldots$

3.10 Variation of the second order shifts with $\theta \ldots \ldots \ldots 1$

3.11 DAS complementary angles $\ldots \ldots \ldots \ldots \ldots \ldots$

3.12 Powder patterns with spinning at DAS complementary angles $\ldots .95$

3.13 Trajectories of the external field viewed in SFC in MAS and DAS . . 96

3.14 DAS experimental setup . . . . . . . . . . . . . . . . 99

3.15 DAS pulse sequence . . . . . . . . . . . . . 100

3.16 DAS rotational echo . . . . . . . . . . . . . . . 104

3.17 One dimensional DAS spectrum . . . . . . . . . . . 106

3.18 Two dimensional DAS spectrum . . . . . . . . . . . . 107

3.19 Two dimensional lineshapes of central transition . . . . . . . . 109

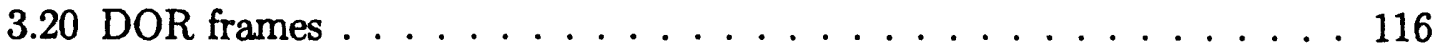

3.21 Residual lineshapes after DOR . . . . . . . . . . . . . . . 118

3.22 Angular momentum diagram in DOR spinners . . . . . . . . 120

3.23 Double-rotor design . . . . . . . . . . . . . . . . . . 122

3.24 Relationship of the spinning speeds of inner and outer rotors . . . . 125

3.25 DOR spectra in a mixture of sodium sulfate and sodium oxalate . . 127

3.26 One cone trajectory to average a single tensor with rank $l \ldots \ldots 130$

3.27 Experimentally measured powder patterns under VASS . . . . . . . 133

3.28 Average of tensors over symmetry point groups $\ldots \ldots \ldots \ldots$

3.29 Two cone trajectory to average out two tensors $\ldots \ldots \ldots 138$

3.30 Continuous one-dimensional trajectory to average two tensors $\ldots . .140$ 
3.31 3D graph of the DAS complementary angles . . . . . . . . . 142

3.32 DAS trajectory related to an icosahedron . . . . . . . . . . 143

4.1 Sideband intensities in MAS . . . . . . . . . . . . . . . 154

4.2 Convergence of the approximate method $\ldots \ldots \ldots \ldots$

4.3 Differences and averages of the sideband intensities $\ldots \ldots \ldots 158$

4.4 Contour graphics of $\delta-\eta$ surfaces in extraction of the CSA tensor . . 160

4.5 Centerband intensity after TOSS . . . . . . . . . . . 161

4.6 DOR sidebands in sodium-23 spectra of sodium oxalate . . . . . . 166

4.7 Schematic variation of simulated sideband intensities as $\omega_{r_{1}}$ changes . 174

4.8 Variations of simulated DOR sideband intensities with rotor phase $\gamma_{r} 175$

4.9 Experimental results of the DOR sideband intensities varying with rotor phase $\gamma_{r} \ldots \ldots \ldots \ldots \ldots \ldots \ldots \ldots \ldots$

4.10 Variation of DOR centerband intensities with $\gamma_{r}$ and $\eta \ldots \ldots 178$

4.11 Sideband suppression by synchronizing the outer rotor in DOR . . . 180

4.12 Variations of the simulated DAS sideband intensities and phases as $\phi$

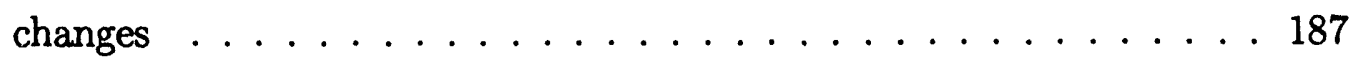

4.13 Variations of the simulated DAS sideband intensities with the sample spinning speed . . . . . . . . . . . . . . . . . . 189

4.14 Simulated DAS spectra using same parameters in DAS experiment of sodium oxalate . . . . . . . . . . . . . . . 191

5.1 Frequencies and linewidths varies with $\kappa$ in two site jumps . . . 196

5.2 Lineshapes varies with $\kappa$ in two site jump model . . . . . . . . 198

5.3 Powder patterns varies with $\kappa$ in two-site jumps . . . . . . . 202

5.4 Variation of lineshapes with tetrahedral jumps . . . . . . . . 220

5.5 Variation of the lineshapes with jumps on a magic angle cone . . . 223

5.6 Definitions of the rotation operators in $I$ group $\ldots \ldots \ldots \ldots$

5.7 Lineshapes with icosahedral jumps under strong collision . . . . . 230 
5.8 Variations of two eigenvalues of the exchange matrix with $\kappa$ in an icosahedral molecule . . . . . . . . . . . . . . . . . . . 235

5.9 Variations of the first two eigenvalues of the exchange matrix with $\kappa$ in an icosahedral molecule . . . . . . . . . . . . . 237

5.10 Variations of the first two eigenvalues of the exchange matrix with $\kappa$ in an icosahedral molecule . . . . . . . . . . . . . 238

5.11 Lineshapes with different jump models in an icosahedral molecule . . 239

5.12 Lineshapes varies with $\kappa$ in a dodecahedral molecule . . . . . . . . 241

5.13 Lineshapes with different jump models in a dodecahedral molecule . . 243

5.14 Structure of a $C_{60}$ molecule $\ldots \ldots \ldots \ldots \ldots \ldots \ldots$

5.15 Variation of powder lineshape in solid $C_{60}$ with $\eta=0 \ldots \ldots 247$

5.16 Variation of powder lineshape in solid $C_{60}$ with $\eta=0.312 \ldots \ldots 248$ 


\section{List of Tables}

1.1 Commutation reiations for $\operatorname{spin} I=1 \ldots \ldots \ldots \ldots$

1.2 Cycling conditions for $\operatorname{spin} I=1 \ldots \ldots \ldots \ldots$

1.3 Zero field evolution matrices, $\mathcal{E}_{m^{\prime}, m}^{l^{\prime}, l}(t)$, for spin $I=1 \ldots \ldots$

1.4 Order selective observation in zero field NMR and NQR . . . . . . 12

1.5 Irreducible tensors in the coupled system with two spin $i=1 \ldots 40$

1.6 Irreducible tensors in the coupled system with two spin $i=1$ (continue) 41

3.1 Values of $\left\langle I\left|T_{i}\right| I\right\rangle \ldots \ldots \ldots \ldots \ldots \ldots \ldots \ldots \ldots \ldots \ldots \ldots$

3.2 Value of $W(2,2, I, I, l, I)$ coefficients. $\ldots \ldots \ldots \ldots \ldots \ldots$

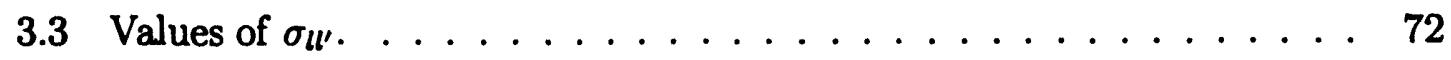

3.4 Singularities appearing in second order powder patterns . . . . . 79

3.5 Angles in an icosahedron or a dodecahedron $\ldots \ldots \ldots \ldots$. . . 97

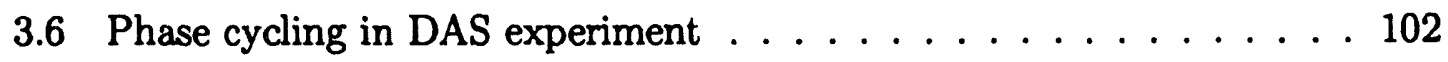

3.7 Roots of Legendre polynomials of $P_{l}\left(\cos \beta^{(l)}\right) \ldots \ldots \ldots \ldots \ldots$

3.8 Characters of $D_{m n}^{(l)}$ representations of the group $S O(3)$ after decomposition by the icosahedral group. . . . . . . . . . . . 135

4.1 Results of sideband analysis $\ldots \ldots \ldots \ldots \ldots \ldots$

5.1 Singularities in a powder lineshape of spin nuclei with chemical anisotropic interaction under two-site jumping . . . . . . . . . . 203

5.2 Characters of the dihedral group $D_{2} \ldots \ldots \ldots \ldots \ldots \ldots \ldots$

5.3 Characters of the tetrahedral group $T, \epsilon=e^{\frac{2 \pi}{3}} \ldots \ldots \ldots 218$ 
5.4 Decomposition of the representations of the tetrahedral group $T$ under the dihedral group $D_{2} \ldots \ldots \ldots \ldots$

5.5 Characters of the dihedral group $D_{3} \ldots \ldots \ldots$

5.6 Characters of the dihedral group $D_{5} \ldots \ldots \ldots$

5.7 Characters of $I$ Group . . . . . . . . . . . . . . . . 227

5.8 Reduction of $\mathcal{D}^{(l)}$ to irreducible representations of $I \ldots \ldots$. . . . . 228

5.9 Decomposition of the representations of the group $I$ under its sub-

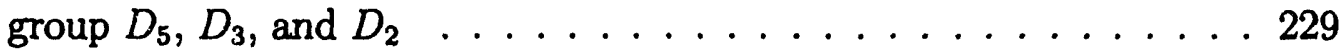

5.10 Orientations at which there are some degree of degeneracy of the resonance frequencies of sites in an icosahedral molecule . . . . . . . 231

5.11 Orientations at which there are some degree of degeneracy of the resonance frequencies of sites in a dodecahedral molecule . . . . . . . . 244 


\section{ACKNOWLEDGMENTS}

It has been my great pleasure since I participated in Professor Alex Pines' research group. In a short space and in such a large group it is impossible for me to properly thank all of giants who helped me in one or other way to finish this work, hence it must suffice to list a few names and extend my gratitude to all the others.

Of course, the special thanks has to go to Professor Alex Pines, my research supervisor, for sharing his insights, idea, energy, encouragement and support without which this work would have been impossible.

Next on my list of acknowledgment must be the members of zero field subgroup, Dr. Dieter Suter, Dr. S. Matsui, Dr. Claudia Schmidt, and Dr. Tom. Jarvie. They introduced me to be interested in zero field multi-dimensional NMR and NQR, and Chapter 1 is the summary of all work together. I have to thank Dr. Ago Samoson, Dr. Z. Peng, Dr. Y. Yu, Dr. C.J. Lee, Dr. K.T. Mueller, Dr. G. Chingas, Dr. Philip Grandinetti, and Professor T. Terao with whom I worked and shared the excitements about one of the most exciting projectors in this group, second order spectroscopy. I also want to thank Dr. Lucio. Frydman who helped me a lot to finish the final chapter: motions in solid $C_{60}$, and Mark Rosen who solved many problems relative to computer programs in all simulations.

Joe Sachlben, Dr. Lucio Frydman, Dan Caplan, and Young Lee helped me to painstakingly do the English correction and proof-reading of this thesis. Without these helps, it is impossible to write up this thesis, I therefore offer them my special thanks, nevertheless, any further complains for typographical and grammatical mistakes in this thesis should be directed to me.

I additionally thank Professor Chaohui Ye for every things that he did for me. Finally, special thanks to my family and my father- and mother-in-law for their encouragement, support, and love throughout my studies, especially my wife, Linggan Qiu, and my father-in-law, Professor $\mathrm{Zu}$-Wen Qiu who devote their lifetime to me. 
This Work is Dedicated to My Father, Song-Yuan Sun, and My Eldest Brother, Bo-Ji Sun, who died during this work. 


\section{Chapter 1}

\section{Zero Field NMR and NQR Spectroscopy}

\subsection{Introduction}

In the development of nuclear magnetic resonarice (NMR) spectroscopy, high sensitivity and resolution are two important goals. In solids, rapid internal random motions are absent so that the local fields created by anisotropic spin interactions (e.g. chemical shift anisotropy, dipolar coupling and quadrupolar coupling etc.) are sensitive to the relative orientations of spins with respect to the external magnetic field $H_{0}$. For a polycrystalline or amorphous solid sample, such an orientation dependence results in the dispersion of the absorption line in NMR spectra and makes not only the interpretation of these spectra difficult[1] (low resolution) but also sensitivity low. The reason why the resonance frequencies of individual spin nuclei become orientation dependent is the truncation of the internal spin interactions by the larger Zeeman interaction. One possible solution to eliminate this dispersion is to remove the external field such that the local fields of all spin nuclei are equivalent and, therefore, orientation independent. This method is commonly used in pure nuclear quadrupolar resonance (NQR) $[2,3,4,5,6]$.

The major obstacle in obtaining NMR spectra at zero magnetic field is the low sensitivity. This sensitivity problem is implied by Faraday's law which states that the 
voltage inducs d in an rf coil by an oscillating magnetization in the xy-plane is proportional to both the amplitude and the resonance frequency of the magnetization. These two factors are linearly dependent on the strength of the external magnetic field, so that the FID signal is proportional the square of the magnetic field. At zero field, the FID's intensity is therefore proportional to the square of the strength of the local magnetic field which is at least a thousand times smaller than the external magnetic field, therefore the sensitivity of the NMR signal is a million times lower than in high field. This severe sensitivity problem may be overcome by using either SQUID techniques [31, 32,33] or by the field cycling method proposed by Pound[7], used first in pure NQR spectroscopy. The most recent application of the field cycling technique is in time-domain zero field NMR and NQR spectroscopy[8]-[12]. In this experiment, the solid sample is transferred pneumatically from the high magnetic field (4.2T) to an intermediate field with the strength about $0.01 T$, and the field is suddenly turned off. The magnetization then evolves for a length of time $t$ in zero field under a scalar spin Hamiltonian (of dipolar or quadrupolar interaction), and is stored along the external field by suddenly turning on the intermediate field. The sample is transferred back into high field where the $\mathrm{z}$ component of the evolved magnetization is observed. The whole FID signal is acquired by increasing the length of time evolved in zero field. After the Fourier transformation, a high resolution spectrum finally may be obtained. The frequencies of the peaks in the spectrum directly determine the principle parameters of the spin interactions involved in zero field. This technique combines the advantage of high resolution and high sensitivity and has shown its power in determining quadrupolar coupling constants of deuterons in molecules.

One would like to advance time-domain zero field NMR and NQR by finding zero field analogs to multi-pulse and multi-dimensional methods originating from high field NMR[11]-[18] to simplify the identification of sites, obtain more structural in- 
formation of molecules, study the motions in molecules, and measure exchange rate between orders. In most of these new experiments, cumbersome calculations are necessary in order to obtain theoretical spectra. In this chapter, a simplified calculation method based on the irreducible tensor theory and Wigner rotation matrices will be described. I then apply this method to discuss the zero field order selective observation, zero field and high field correlation spectroscopy, and the measurement of spin diffusion in zero field.

\subsection{Calculation of Zero Field NMR and NQR Spectra}

One of the differences between zero field and high field NMR is that the eigenvalues of the spin Hamiltonian do not depend on the orientation of the local principal axis system of a spin interaction with respect to the laboratory frame[9]. For this reason, zero field NMR spectra usually show high resolution. One can then ask how the orientation distribution of a powder sample affects the spectrum. The answer to this question is that the eigenvectors of the spin Hamiltonian, and thus the transition intensities are orientation dependent. However, for some complicated pulse sequences in zero field, the common procedure for taking a powder average is complicated due

to many integrations over trigonometric functions. In this section, we will describe a general method which can greatly simplify the calculation of the zero field NMR and NQR spectra by using the irreducible tensor theory and properties of Wigner rotation matrices[20,21, 22, 23, 24]. We will show some particular cases where the theoretical spectrum after a particular pulse sequence can be straightforwardly calculated by our method without performing any detailed integrations in the powder average.

We start with the spin Hamiltonian of a quadrupolar interaction in its principle axis system, given by[19]

$$
\mathcal{H}=\rho_{2,0} T_{2,0}+\rho_{2,2}\left(T_{2,2}+T_{2,-2}\right)
$$


where the $T_{2, m}$ are the components of a second-rank irreducible spin tensor defined by

$$
\begin{aligned}
T_{2,0} & =\frac{1}{\sqrt{6}}\left(3 I_{z}^{2}-I^{2}\right) \\
T_{2, \pm 1} & =\mp \frac{1}{2}\left(I_{z} I_{ \pm}+I_{ \pm} I_{z}\right) \\
T_{2, \pm 2} & =\frac{1}{2} I_{ \pm} I_{ \pm}
\end{aligned}
$$

and

$$
I_{ \pm}=\mp \frac{1}{\sqrt{2}}\left(I_{x} \pm \imath I_{y}\right)
$$

The $\rho_{2, m}$ are the principal components of the second-rank ineducible spatial tensor of the quadrupolar interaction, given by

$$
\rho_{2,0}=\sqrt{\frac{3}{2}} \delta, \quad \rho_{2, \pm 1}=0, \quad \text { and } \rho_{2, \pm 2}=\frac{1}{2} \delta \eta
$$

where for our case

$$
\delta=\omega_{Q}=\frac{e^{2} q Q}{2 I(2 I+1)}
$$

and $Q$ is the quadrupolar moment of the nucleus, $q$ is the strength of the electric field gradient in the $\mathrm{z}$ direction, $e$ is the electron charge, and $I$ is the nuclear spin value.

The initial state of the density matrix also can be expressed in terms of the irreducible tensors as

$$
\rho(0)=\sum_{l, m} a_{l, m} T_{l, m},
$$

where $a_{l, m}$ is a coefficient which means percentage of total population in the order of $T_{l, m}$ given in the initial preparation period. From the normalization property of the density matrix, which is equivalent to the energy conservation requirement for the whole system, we have

$$
\sum_{l, m} a_{l, m} a_{l, m}^{\dagger}=\frac{1}{C_{N}(l, I)}
$$


where $C_{N}(l, I)$ is a normalization constant, which is a function of the rank of the irreducible tensor and spin value, given by[21]

$$
C_{N}(l, I)=\frac{1}{2 l+1} \frac{l ! l !(2 I+l+1) !}{2^{l}(2 l) !(2 I-l) !} .
$$

In the derivation of Eq.(1.7) we have used orthogonality properties of the irreducible tensors

$$
\operatorname{Tr}\left\{T_{l, m} T_{l^{\prime}, m^{\prime}}^{\dagger}\right\}=C_{N}(l, I) \delta_{l, l^{\prime}} \delta_{m, m^{\prime}}
$$

With Eqs.(1.1) and (1.6), the evolution of the density matrix under the quadrupolar Hamiltonian during time $t$ is

$$
\rho(t)=e^{-\imath \mathcal{H} t} \rho(0) e^{\imath \mathcal{H} t}=\sum_{l, m} a_{l, m} \sum_{l^{\prime}, m^{\prime}} \mathcal{E}_{m^{\prime}, m}^{l^{\prime}, l}(t) T_{l^{\prime}, m^{\prime}},
$$

where the $\mathcal{E}_{m^{\prime}, m}^{l^{\prime}, l}(t)$ are the elements of the evolution matrices of $l$-th rank tensor, determined by the spin Hamiltonian. Its explicit representation in general depends on anisotropic parameters, $\delta$ and $\eta$, of the interaction, the spin operators in the spin Hamiltonian, the spin value, and the initial condition of the spin density matrix. If the initial order of the density matrix is zero, because the spin Hamiltonian always commutes with unit operator the only value of the evolution matrices is one, that is

$$
\mathcal{E}_{0,0}^{0,0}(t)=1
$$

Another trivial solution is that at time zero it is diagonal.

$$
\mathcal{E}_{m^{\prime}, m}^{l^{\prime}, l}(0)=\delta_{l^{\prime}, l} \delta_{m^{\prime}, m}
$$

It is obviously orthonormal.

$$
\sum_{l_{1}, m_{1}} \mathcal{E}_{m_{1}, m}^{l_{1}, l}(t) \mathcal{E}_{m_{2}, m_{1}}^{l_{2, l_{1}}}{ }^{\dagger}(t)=\delta_{l, l_{2}} \delta_{m, m_{2}}
$$

and the evolution matrix at time $t_{1}$ and $t_{2}$ is given by

$$
\sum_{l_{1}, m_{1}} \mathcal{E}_{m_{1}, m}^{l_{1}, l}\left(t_{1}\right) \mathcal{E}_{m_{2}, m_{1}}^{l_{2}, l_{1}}\left(t_{2}\right)=\mathcal{E}_{m_{2, m}}^{l_{2}, l}\left(t_{1}+t_{2}\right)
$$


If the spin operators in the spin Hamiltonian are linear (e.g. the Hamiltonian of Zeeman interaction, or chemical shift interaction), the matrix elements of the evolution matrices are

$$
\mathcal{E}_{m^{\prime}, m}^{l^{\prime}, l}(t)=\delta_{l^{\prime}, l} \delta_{m^{\prime}, m} e^{\imath m \omega_{i} t}
$$

where $\omega_{i}$ is the strength of $i$-th interaction.

Calculating the evolution matrices becomes very difficult when the spin Hamiltonian contains bilinear spin operators, and in this case, there is no general solution for an arbitrary value of spin. However the matrix representations still can be obtained for small values of the spin on a case by case basis. As a simple example, let us consider a spin $I=1$ system with a quadrupolar interaction. The highest rank irreducible tensor necessary in expansion of the density matrix is two. Thus, the zero-, first-, and second-rank tensors provide a complete set of basis operators in the evolution of the density matrix. With the properties of the irreducible tensors[20], we can derive the following general commutator relationship:

$$
\left[T_{l_{1}, m_{1}}, T_{l_{2}, m_{2}}\right]=\sum_{l} C\left(l_{1}, l_{2}, l, m_{1}, m_{2}\right)\left[1-(-1)^{l_{1}+l_{2}-l}\right] T_{l, m_{1}+m_{2}} .
$$

Table (1.1) shows all commutation relationships for spin $I=1$. By Table (1.1), it is easy to prove that

$$
\left[T_{2,0},\left(T_{2,2}+T_{2,-2}\right)\right]=0 .
$$

This last equation allows us to separately derive the evolution matrices for the $T_{2,0}$, and $\left(T_{2,2}+T_{2,-2}\right)$ terms in the Hamiltonian (Eq. 1.1). These two evolution matrices are combined to obtain the final matrix elements of the evolution matrices.

In the next step to calculate the evolution matrices $\mathcal{E}_{m^{\prime}, m}^{l^{\prime}, l}(t)$, we need to consider a function of operators

$$
f(\theta)=e^{-\mathbf{\imath} \hat{\mathbf{A}} \theta} \hat{\mathbf{B}} e^{i \hat{\mathbf{A}} \theta},
$$

where $\hat{\mathbf{A}}$ and $\hat{\mathbf{B}}$ could be any two operators. The derivatives of the function $f(\theta)$ are

$$
\frac{d}{d \theta} f(\theta)=-\imath e^{-\imath \hat{\mathbf{A}} \theta}[\hat{\mathbf{A}}, \hat{\mathbf{B}}] e^{\imath \hat{\mathbf{A}} \theta}
$$


Table 1.1: Commutation relations for spin $I=1$

\begin{tabular}{|c|c|}
\hline \hline$\left[T_{1,0}, T_{l, m}\right]=m T_{l, m}$ & {$\left[T_{2,0}, T_{2, \pm 1}\right]= \pm \frac{\sqrt{3}}{2} T_{1, \pm 1}$} \\
{$\left[T_{1,1}, T_{1,-1}\right]=-T_{1,0}$} & {$\left[T_{2,1}, T_{2,-1}\right]=-\frac{1}{2} T_{1,0}$} \\
{$\left[T_{1, \pm 1}, T_{2, \pm 1}\right]=\mp \sqrt{2} T_{2, \pm 2}$} & {$\left[T_{2, \pm 1}, T_{2, \mp 2}\right]= \pm \frac{1}{\sqrt{2}} T_{1, \mp 1}$} \\
{$\left[T_{1, \pm 1}, T_{2, \mp 1}\right]=\mp \sqrt{3} T_{2,0}$} & {$\left[T_{2,2}, T_{2,-2}\right]=T_{1,0}$} \\
{$\left[T_{1, \pm 1}, T_{2, \mp 2}\right]=\mp \sqrt{2} T_{2, \mp 1}$} & {$\left[T_{1, \pm 1}, T_{2,0}\right]=\mp \sqrt{3} T_{2, \pm 1}$} \\
\hline
\end{tabular}

Other commutators not shown, are identicaliy equal to zero.

$$
\begin{aligned}
\frac{d^{2}}{d \theta^{2}} f(\theta) & =(-\imath)^{2} e^{-\imath \hat{\mathbf{A}} \theta}[\hat{\mathbf{A}},[\hat{\mathbf{A}}, \hat{\mathbf{B}}]] e^{\imath \hat{\mathbf{A}} \theta} \\
\frac{d^{3}}{d \theta^{3}} f(\theta) & =(-\imath)^{3} e^{-\imath \hat{\mathbf{A}} \theta}[\hat{\mathbf{A}},[\hat{\mathbf{A}},[\hat{\mathbf{A}}, \hat{\mathbf{B}}]]] e^{2 \hat{\mathbf{A}} \theta}
\end{aligned}
$$

and the Taylor expansion of the function $f(\theta)$ is

$$
f(\theta)=\hat{\mathbf{B}}-\imath \theta[\hat{\mathbf{A}}, \hat{\mathbf{B}}]+\frac{1}{2 !}(-\imath \theta)^{2}[\hat{\mathbf{A}},[\hat{\mathbf{A}}, \hat{\mathbf{B}}]]+\frac{1}{3 !}(-\imath \theta)^{3}[\hat{\mathbf{A}},[\hat{\mathbf{A}},[\hat{\mathbf{A}}, \hat{\mathbf{B}}]]]+\cdots
$$

In our example the operator $\hat{\mathbf{A}}$ can be either $T_{2,0}$ or $T_{2,2}+T_{2,-2}$, and $\hat{\mathbf{B}}$ is the whole set of the irreducible tensors from rank zero to two respectively. From Eq.(1.20) and the commutation relationships of the irreducible tensors which are listed in Table (1.1), cycling conditions can be found after the second derivatives of the function $f(\theta)$ for spin $I=1$. These cycling conditions are tabulated in Table (1.2). From the cycling conditions components of the evolution matrices are straightforwardly calculated. These components are tabulated in Table (1.3). By following the same procedure above, evolution matrices can be tediously derived one by one for all other spin values.

Next we need to determine the response of the spin system to a dc pulse. It is well known that a pulse applied on a spin operator behaves like a rotation operator. In order to use the Wigner rotation matrices as a representation of the pulse response, 
Table 1.2: Cycling conditions for spin $I=1$

\begin{aligned} & \hline$e^{-\imath \theta T_{2,0}} T_{1,0} e^{\imath \theta T_{2,0}}=T_{1,0} \\ & e^{-\imath \theta T_{2,0}} T_{2,0} e^{\imath \theta T_{2,0}}=T_{2,0} \\ & e^{-\imath \theta i 2,0}\left(T_{2,2}+T_{2,-2}\right) e^{\imath \theta T_{2,0}}=\left(T_{2,2}+T_{2,-2}\right) \\ & e^{-\imath \theta T_{2,0}}\left(T_{2,2}-T_{2,-2}\right) e^{\imath \theta T_{2,0}}=\left(T_{2,2}-T_{2,-2}\right) \\ &$\hline$\frac{d^{2}}{d \theta^{2}}\left[e^{-\imath \theta T_{2,0}} T_{1, \pm 1} e^{\imath \theta T_{2,0}}\right]=(-\imath)^{2} \frac{3}{2} T_{1, \pm 1} \\ & \frac{d^{2}}{d \theta^{2}}\left[e^{-\imath \theta T_{2,0}} T_{2, \pm 1} e^{\imath \theta T_{2,0}}\right]=(-\imath)^{2} \frac{3}{2} T_{2, \pm 1} \\ &$\hline$e^{-\imath \theta\left(T_{2,2}+T_{2,-2}\right)} T_{2,0} e^{\imath \theta\left(T_{2,2}+T_{2,-2}\right)}=T_{2,0} \\ & e^{-\imath \theta\left(T_{2,2}+T_{2,-2}\right)}\left(T_{2,2}+T_{2,-2}\right) e^{\imath \theta\left(T_{2,2}+T_{2,-2}\right)}=\left(T_{2,2}+T_{2,-2}\right) \\ &$\hline$\frac{d^{2}}{d \theta^{2}}\left[e^{-\imath \theta\left(T_{2,2}+T_{2,-2}\right)} T_{1,0} e^{\imath \theta\left(T_{2,2}+T_{2,-2}\right)}\right]=(-\imath)^{2} 4 T_{1,0} \\ & \frac{d^{2}}{d \theta^{2}}\left[e^{-\imath \theta\left(T_{2,2}+T_{2,-2}\right)} T_{1, \pm 1} e^{\imath \theta\left(T_{2,2}+T_{2,-2}\right)}\right]=(-\imath)^{2} T_{1, \pm 1} \\ & \frac{d^{2}}{d \theta^{2}}\left[e^{-\imath \theta\left(T_{2,2}+T_{2,-2}\right)} T_{2, \pm 1} e^{\imath \theta\left(T_{2,2}+T_{2,-2}\right)}\right]=(-\imath)^{2} T_{2, \pm 1} \\ & \frac{d^{2}}{d \theta^{2}}\left[e^{-\imath \theta\left(T_{2,2}+T_{2,-2)}\right)}\left(T_{2,2}-T_{2,-2}\right) e^{\imath \theta\left(T_{2,2}+T_{2,-2}\right)}\right]=(-\imath)^{2} 4\left(T_{2,2}-T_{2,-2}\right) \\ &$\hline\end{aligned}

we represent the dc pulse, and equivalently, the rotation operator, according to Euler angles, and the spin operators by irreducible tensors. We will call the rotation operator $\mathbf{P}\left(\Omega_{P}\right)$. As an explicit example, the initial density matrix is given by Eq.(1.6) in the laboratory frame, but after a dc pulse it becomes

$$
\rho\left(0_{+}\right)=\sum_{l, m} a_{l, m} \sum_{m^{\prime}} \mathcal{D}_{m^{\prime}, m}^{(l)}\left(\Omega_{P}\right) T_{l, m^{\prime}}
$$

In many cases the initial density matrix is represented in the molecular frame. The representation of the pulse therefore has to be transferred into the molecular frame, and the response of the spin density matrix to the pulse shows orientation dependence. We use $\mathcal{P}_{m^{\prime}, m}^{(l)}\left(\Omega_{M}, \Omega_{P}\right)$ to be the matrix elements of pulse rotation operator in the molecular frame. This representation can be related to the Wigner rotation matrices in the following way: the spin density matrix is rotated to the laboratory frame where it is operated on by the dc pulse, and then is rotated back into the 
Table 1.3: Zero field evolution matrices, $\mathcal{E}_{m^{\prime}, m}^{l^{\prime}, l}(t)$, for spin $I=1$

\begin{tabular}{||c|c|c|c||c|c|c|c|c||}
\hline \hline$l, m$ & \multicolumn{10}{|c||}{$l^{\prime}, m^{\prime}$} \\
\hline & 1,1 & 1,0 & $1,-1$ & 2,2 & 2,1 & 2,0 & $2,-1$ & $2,-2$ \\
\hline 1,1 & $\frac{1}{2} C_{+}$ & 0 & $\frac{-1}{2} C_{-}$ & 0 & $\frac{-2}{\sqrt{2}} S_{+}$ & 0 & $\frac{-2}{\sqrt{2}} S_{-}$ & 0 \\
\hline 1,0 & 0 & $C_{0}$ & 0 & $2 S_{0}$ & 0 & 0 & 0 & $-\imath S_{0}$ \\
\hline $1,-1$ & $\frac{-1}{2} C_{-}$ & 0 & $\frac{1}{2} C_{+}$ & 0 & $\frac{2}{\sqrt{2}} S_{-}$ & 0 & $\frac{2}{\sqrt{2}} S_{+}$ & 0 \\
\hline 2,2 & 0 & $\frac{2}{2} S_{0}$ & 0 & $\frac{1}{2}+\frac{1}{2} C_{0}$ & 0 & 0 & 0 & $\frac{1}{2}-\frac{1}{2} C_{0}$ \\
\hline 2,1 & $\frac{2}{2 \sqrt{2}} S_{+}$ & 0 & $\frac{2}{2 \sqrt{2}} S_{-}$ & 0 & $\frac{1}{2} C_{+}$ & 0 & $\frac{-1}{2} C_{-}$ & 0 \\
\hline 2,0 & 0 & 0 & 0 & 0 & 0 & 1 & 0 & 0 \\
\hline $2,-1$ & $\frac{-2}{2 \sqrt{2}} S_{-}$ & 0 & $\frac{-1}{2 \sqrt{2}} S_{+}$ & 0 & $\frac{-1}{2} C_{-}$ & 0 & $\frac{1}{2} C_{+}$ & 0 \\
\hline $2,-2$ & 0 & $\frac{-2}{2} S_{0}$ & 0 & $\frac{1}{2}-\frac{1}{2} C_{0}$ & 0 & 0 & 0 & $\frac{1}{2}+\frac{1}{2} C_{0}$ \\
\hline
\end{tabular}

In the table, $C_{+}=\cos \left(\omega_{+} t\right)+\cos \left(\omega_{-} t\right), C_{0}=\cos \left(\eta \omega_{Q} t\right), C_{-}=\cos \left(\omega_{+} t\right)-$ $\cos \left(\omega_{-} t\right), S_{+}=\sin \left(\omega_{+} t\right)+\sin \left(\omega_{-} t\right), S_{0}=\sin \left(\eta \omega_{Q} t\right), S_{-}=\sin \left(\omega_{+} t\right)-$ $\sin \left(\omega_{-} t\right), \omega_{+}=\frac{3+\eta}{2} \omega_{Q}, \omega_{-}=\frac{3-\eta}{2} \omega_{Q}$.

molecular frame. After this procedure, the pulse rotation operator is given by

$$
\mathcal{P}_{m^{\prime}, m}^{l}\left(\Omega, \Omega_{P}\right)=\sum_{m_{1}, m_{2}} \mathcal{D}_{m, m_{1}}^{(l)}{ }^{\dagger}(\Omega) \mathcal{D}_{m_{2}, m_{1}}^{(l)}\left(\Omega_{P}\right) \mathcal{D}_{m^{\prime}, m_{2}}^{(l)}(\Omega)
$$

Usually a pulse is represented by the pulse length $\theta$ and the phase $\varphi$ of the rotation axis relative to the $x$ axis of the laboratory frame, (in high field case, of the rotating frame).

Once the initial condition and the evolution rules of the density matrix have been determined, we transfer the density matrix from the laboratory frame to the principle axis system of the interaction tensor (if there is more than one interaction in the Hamiltonian, a molecule frame has to be introduced into the calculation, but now we deal only with a single interaction). In the principle axis system, the initial density matrix is

$$
\rho_{P}(0)=\sum_{l, m} a_{l, m} \sum_{m^{\prime}} \mathcal{D}_{m^{\prime}, m}^{(l)}(\Omega) T_{l, m^{\prime}}
$$


The observable, $\mathrm{O}$, can also be represented into the same form as $\mathrm{Eq} .(1.23)$.

$$
\mathbf{O}=\sum_{l, m} b_{l, m} \sum_{m^{\prime}} \mathcal{D}_{m^{\prime}, m}^{(l)}(\Omega) T_{l, m^{\prime}}
$$

After inserting Eq.(1.23) into Eq.(1.10) and with Eq.(1.24), the FID signal will be

$$
\begin{aligned}
g(t, \Omega) & =\operatorname{Tr}[\rho(t) O] \\
& =\sum_{l_{1}, l_{2}, l_{1}, m_{1}, m_{2}, m_{3}, m_{4}, m} a_{l_{1}, m_{1}} b_{l, m} \mathcal{D}_{m_{2}, m_{1}}^{\left(l_{1}\right)}(\Omega) \mathcal{D}_{m_{3}, m_{4}}^{*\left(l_{2}\right)}(\Omega) \mathcal{E}_{m_{3}, m_{2}}^{l_{2}, l_{1}} \operatorname{Tr}\left\{T_{l, m} T_{l_{2}, m_{4}}\right\}
\end{aligned}
$$

The orthogonal properties of the Wigner rotation matrices and irreducible tensors imply that the powder averaged FID signal will be

$$
G(t)=\sum_{l_{1}, m_{1}, m_{2}} \frac{(-1)^{m_{1}}}{2 l_{1}+1} a_{l_{1}, m_{1}} b_{l_{1},-m_{1}} \mathcal{E}_{m_{2}, m_{2}}^{l_{1}, l_{1}} C_{N}\left(l_{1}, I\right)
$$

where $C_{N}(l, I)$ is a normalization constant given by Eq.(1.8). As we can see from Eq.(1.26), the evolution matrix, the initial condition of the density matrix, the observable, and Clebsch-Gordan coefficients totally determine the powder averaged spectrum. We do not need to perform any integrations over orientations. Another interesting point is that the observable is actually determined by the initial order in the density matrix, irrespective of what other orders are involved during the evolution time under the Hamiltonian.

As a simple example, let consider the sudden switch experiment for spin $I=1$. Both the initial state and the observable are represented by $I_{z}=T_{1,0}$, and $a_{1,0}=$ $b_{1,0}=1$ in the laboratory frame. From Eq.(1.26) and Table (1.3), the FID signal is then

$$
G(t)=\frac{1}{3}\left[\cos \left(\omega_{Q} \eta t\right)+\cos \left(\frac{3-\eta}{2} \omega_{Q} t\right)+\cos \left(\frac{3+\eta}{2} \omega_{Q} t\right)\right] .
$$

Other examples will be given in following sections. It should be pointed out that Table (1.3) can also be used for two coupled spins with spin value $I=\frac{1}{2}$. 


\subsection{Order Selective Observation of Zero Field Signals}

In zero field NMR and NQR spectroscopy, a single spin $I=1$ or pseudo-spin-1 system has four nuclear transitions. Each transition corresponds to a peak in a spectrum. The spectrum will eventually become very complicated as the number of spin-1's nuclei in a molecule increases, and if each spin-1 nucleus has different value of the quadrupolar (or dipolar) coupling constant and the asymmetry parameter. The assignment of each peak in the spectrum therefore becomes very difficult. In high field NMR, there are several methods to simplify a complicated spectrum [30], one of them is multiple quantum transition NMR spectroscopy $[25,26,27,28,30]$. In this method, a multiple quantum operator (pumping operator in optics) which is a linear combination of a set of different rank tensors is created by a particular pulse sequence [25]. The $n$-quantum coherence in the density matrix is then prepared by applying the multiple quantum operator to the spin system for length of times, $\tau$, where the density matrix starts to evolve under the spin Hamiltonian. At the end of the evolution, another multiple quantum operator is applied to observe multiple quantum transitions. The second multiple quantum operator is usually the conjugate operator of the first multiple quantum operator in order to obtain an in-phase spectrum with multiple transitions. The idea of the multiple quantum NMR can also be used selectively to observe the zero field signal, and we call this experiment the order selective observation. In the order selective observation of zero field NMR signal, we must prepare a particular initial order for the density matrix by using a dc pulse sequence. This particular order can be completely represented by an irreducible tensor. In similar principle, we need another particular order to observe the evolution of the density matrix in zero field. The zero field NMR signal therefore depends on the selection of the initial order and the observi-g order. In this section we will show four different initial and observable orders. Each of them will yield different signal 
forms, and the linear combination of these signals can greatly simplify the spectrum.

The initial density matrix can be represented according to the orders of the population distribution and each individual orders $\left(m_{l}\right)$ be expressed by the $m$-th components of $l$-th rank tensors, or their linear combinations. For example in the sudden switch experiment, the initial density matrix only contains the first order (sometimes is called Zeeman order) for $m=0$ which is represented by $T_{1,0}$ of the first-rank irreducible spin tensor, while in the adiabatic demagnetization experiment, the initial density matrix has the second order: quadrupolar order (or dipolar order) for $m=0$ and $\eta$ order for $m= \pm 2$, represented by the second rank irreducible tensor, $T_{2,0}$ and $T_{2,2}+T_{2,-2}$ respectively. Similarly the observable also has Zeeman order, quadrupolar order, and $\eta$ order. The linear combination of the initial preparation and the observation of the spin density matrix gives us four different choices to observe the zero field NMR signals for a spin $I=1$ system.

Table 1.4: FID signals with different order selective observations in zero field NMR and NQR for a powder sample with spin $I=1$.

\begin{tabular}{|c|c|c|}
\hline \hline Initial & Observable & FID Signal \\
\hline$Z$ & $Z$ & $\frac{1}{3}\left[\cos \left(\omega_{\eta} t\right)+\cos \left(\omega_{-} t\right)+\cos \left(\omega_{+} t\right)\right]$ \\
\hline$Q+\eta$ & $Z$ & $\frac{1}{3}(2 \sin 2 \theta+\sin \theta)\left[a_{-} \sin \left(\omega_{-} t\right)+a_{+} \sin \left(\omega_{+} t\right)\right]$ \\
\hline$Z$ & $Q+\eta$ & $\frac{1}{3}(2 \sin 2 \theta+\sin \theta)\left[a_{-} \sin \left(\omega_{-} t\right)+a_{+} \sin \left(\omega_{+} t\right)\right]$ \\
\hline$Q+\eta$ & $Q+\eta$ & $p_{0}(\theta)+p_{\eta}(\theta) \cos \left(\omega_{\eta} t\right)+p_{-}(\theta) \cos \left(\omega_{-} t\right)+p_{+}(\theta) \cos \left(\omega_{+} t\right)$ \\
\hline
\end{tabular}

where $p_{0}(\theta), p_{\eta}(\theta), p_{-}(\theta)$, and $p_{+}(\theta)$ are given by Eq.(1.28) in text as the pulse response. $a_{ \pm}=\sqrt{6} a_{2,0} \pm\left(a_{2,2}+a_{2,-2}\right)$, where $a_{2,0}, a_{2,2}$, and $a_{2,-2}$ are the constants to form the quadrupolar order and $\eta$ order in PAS.

From Eq.(1.8), for an experiment which only consists of the initial preparation and free evolution under the quadrupolar Hamiltonian, the FID signal before the powder average is given by Eq.(1.25). The powder average depends on the initial order of the 

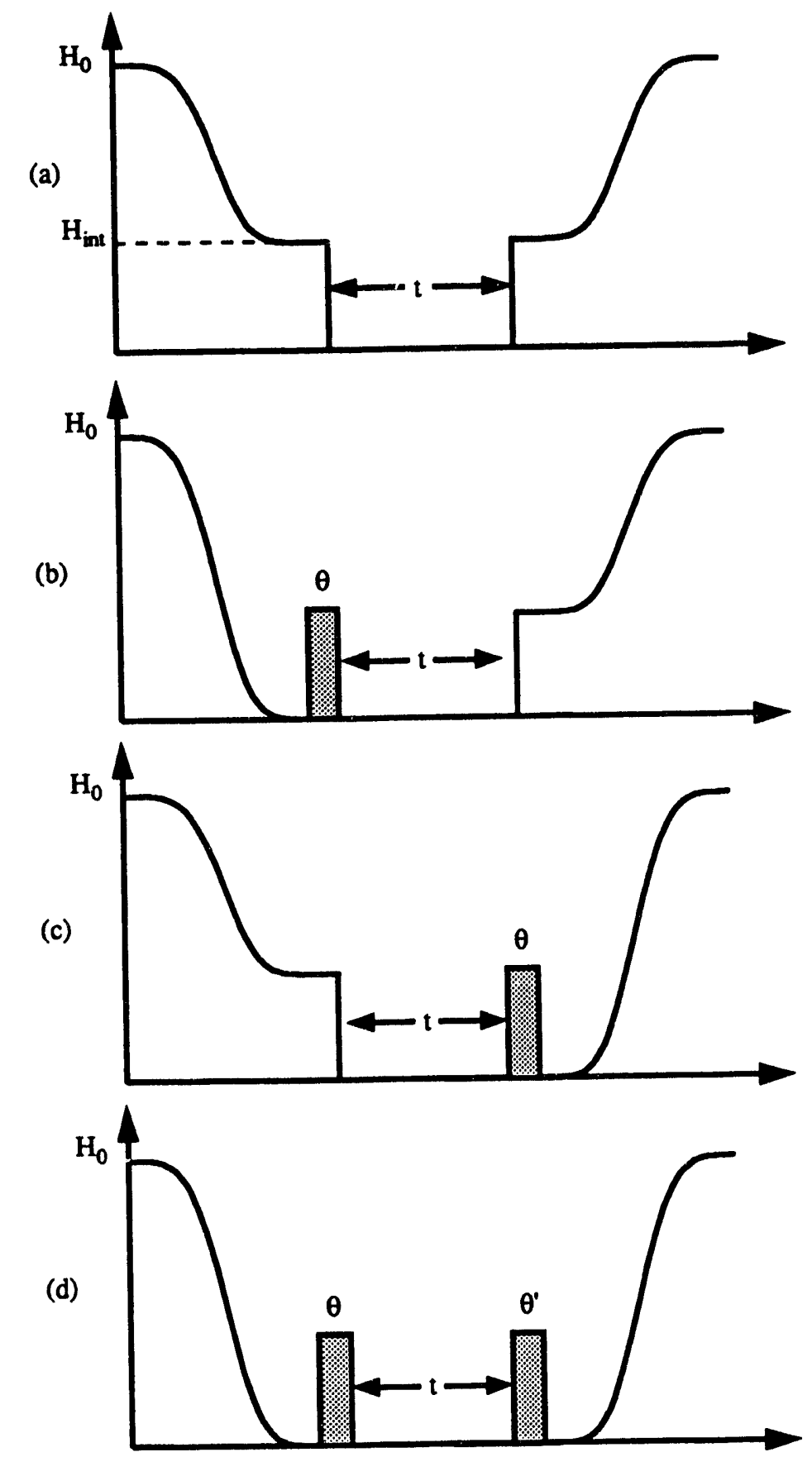

Figure 1.1: Initial preparation and observation of the zero field NMR signal in field cycling experiment. (a) Sudden switch experiment in which both the initial and observable orders are Zeeman order; (b) adiabatic demagnetization experiment in which quadrupolar (or dipolar order) and $\eta$ order are prepared initial, but the observable is the Zeeman order; (c) the initial and observable orders of (b) are replaced with each other; (d) both the initial and observable orders are quadrupolar and $\eta$ order, where $\theta$ and $\theta^{\prime}$ are dc pulse lengths and experimentally we choose $\theta=\theta^{\prime}$ or $\theta=-\theta^{\prime}$. 
density matrix and the order of the observable. For the four configurations shown in Figure (1.1), the powder averaged FID signals are listed in Table (1.4). For the case in which both the initial density matrix and observable are the quadrupolar and $\eta$ orders, the pulse response becomes very complicated and the four coefficients in the last row in Table (1.4) are expressed as

$$
\begin{aligned}
p_{0}(\theta) & =\frac{1}{2} \sum_{l=0}^{4} f_{l}(\theta) \sum_{m=-2}^{2} \sum_{m^{\prime}=-1}^{1}(-1)^{m} a_{2, m}\left[(-1)^{l} a_{2,-m} C^{2}\left(22 l, m, 2 m^{\prime}\right)\right. \\
& \left.+a_{2,4 m^{\prime}-m} C\left(22 l, m,-2 m^{\prime}\right) C\left(22 l, 2 m^{\prime}, m-4 m^{\prime}\right)\right] \\
p_{\eta}(\theta) & =\frac{1}{2} \sum_{l=0}^{4} f_{l}(\theta) \sum_{m=-2}^{2} \sum_{m^{\prime}=-1,1}(-1)^{m} a_{2, m}\left[(-1)^{l} a_{2,-m} C^{2}\left(22 l, m, 2 m^{\prime}\right)\right. \\
& \left.-a_{2,4 m^{\prime}-m} C\left(22 l, m,-2 m^{\prime}\right) C\left(22 l, 2 m^{\prime}, m-4 m^{\prime}\right)\right] \\
p_{+}(\theta) & =\frac{1}{2} \sum_{l=0}^{4} f_{l}(\theta) \sum_{m=-2}^{2} \sum_{m^{\prime}=-1,1}(-1)^{m} a_{2, m}\left[(-1)^{l} a_{2,-m} C^{2}\left(22 l, m, m^{\prime}\right)\right. \\
& \left.+a_{2,2 m^{\prime}-m} C\left(22 l, m,-m^{\prime}\right) C\left(22 l, m^{\prime}, m-2 m^{\prime}\right)\right] \\
p_{-}(\theta) & =\frac{1}{2} \sum_{l=0}^{4} f_{l}(\theta) \sum_{m=-2}^{2} \sum_{m^{\prime}=-1,1}(-1)^{m} a_{2, m}\left[(-1)^{l} a_{2,-m} C^{2}\left(22 l, m, m^{\prime}\right)\right. \\
& \left.-a_{2,2 m^{\prime}-m} C\left(22 l, m,-m^{\prime}\right) C\left(22 l, m^{\prime}, m-2 m^{\prime}\right)\right] \\
f_{l}(\theta) & =\frac{1}{l+1}\left[1+2 \sum_{k=1}^{l} \cos (k \theta)\right] .
\end{aligned}
$$

If the demagnetization in the experiment is adiabatic, the initial order of the density matrix before applying the dc pulse is quadrupolar and in the principle axis system the density matrix should be orientation independent and the coefficients $a_{2, m}$ have the values $a_{2,0}=\sqrt{6} / 2$ and $a_{2, \pm 2}=1 / 2$. Based on this assumption we have plotted the pulse response in Figure (1.2a). For comparison, the pulse response for the conjugate quadrupolar order and $\eta$ order, $(Q+\eta)^{\dagger}$, to obtain an in-phase spectrum during detection are plotted in Figure (1.2b).

From Table (1.4) and Figure (1.2), we can conclude the following. When both the initial and the observing orders are Zeeman order, there are three peaks with equal intensity in zero field spectra and no non-evolved peak at zero frequency. If there is a zero frequency peak, it means that there are some other orders, appearing both in the initial density matrix and in the observable, if the speed of turning off 
the intermediate field is not fast enough. However, when the initial density matrix is determined by the quadrupolar and $\eta$ orders and the observable is Zeeman order, the peaks at $\omega_{\eta}$ and zero frequency disappear. The transitions with frequency $\omega_{+}$ and $\omega_{-}$appear in the dispersive mode and have an intensity ratio of $2: 1$. In the case where the initial density matrix and observable are both quadrupolar and $\eta$ orders, the intensity of one or two from total four transitions may be zero by properly choosing pulse length. For example, when $\theta=68^{\circ}$, the $\omega_{-}$transition is zero and $\omega_{+}$ transition contain more than one quarter of the total intensity, and the $\eta$ transition is very small. Experimentally, it is therefore possible to edit the above three different spectra such that each edited spectrum contains only one kind of transition once the pulse length is known. We can also measure the coefficients $a_{2, m}$ of the initial order by varying the pulse length. This experiment will allow us to confirm the above assumption and to assertion the adiabaticity of the demagnetization process.

Although the order selective observation provides a way to distinguish the different transitions by the different pulse response for same kind of spin nuclei, it does not identify transitions which come from different kinds of nuclei. In order to separate transitions which come from different sites in the sample, a two dimensional correlation spectroscopy may be used. The simplest pulse sequence for obtaining a two dimensional spectrum consists of a single pulse which is applied at the end of the first evolution period. In the principle axis system of the quadrupolar interaction tensor, this pulse becomes orientation dependent. Again this orientation dependence is given by $\mathcal{P}_{m^{\prime}, m}^{(l)}$ of Eq. (1.30). Before the pulse is applied the density matrix in the principle axis system is

$$
\rho\left(t_{-}\right)=\sum_{l_{1}, l_{2}, m_{1}, m_{2}, m_{3}} a_{l_{1}, m_{1}} \mathcal{D}_{m_{2}, m_{1}}^{\left(l_{1}\right)}(\Omega) \mathcal{E}_{m_{3}, m_{2}}^{l_{1}, l_{2}}\left(t_{-}\right) T_{l_{2}, m_{3}},
$$

After application of the dc pulse, the density matrix becomes

$$
\rho\left(t_{+}\right)=\sum_{l_{1}, l_{2}, m_{1}, m_{2}, m_{3}, m_{4}} a_{l_{1}, m_{1}} \mathcal{D}_{m_{2}, m_{1}}^{\left(l_{1}\right)}(\Omega) \mathcal{E}_{m_{3}, m_{2}}^{l_{1}, l_{2}}\left(t_{+}\right) \mathcal{P}_{m_{4}, m_{3}}^{l_{2}}\left(\Omega, \Omega_{P}\right) T_{l_{2}, m_{4}}
$$


(a)

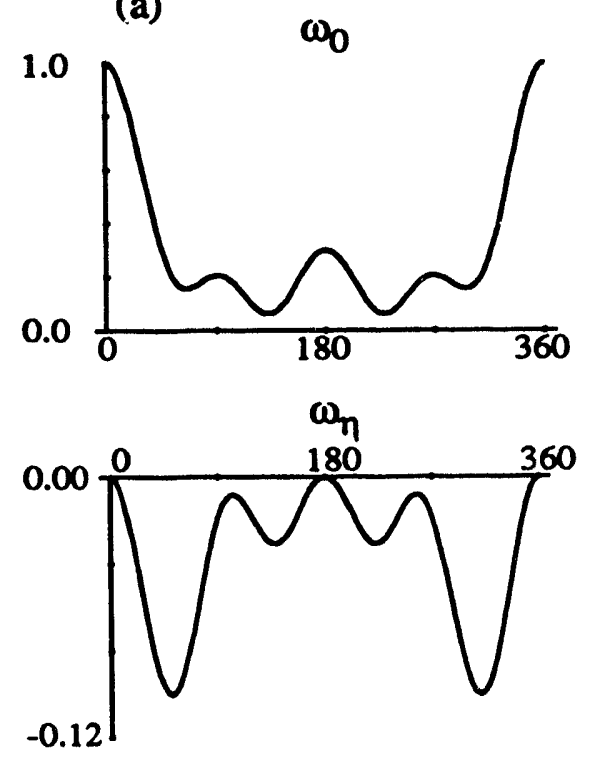

$\omega$
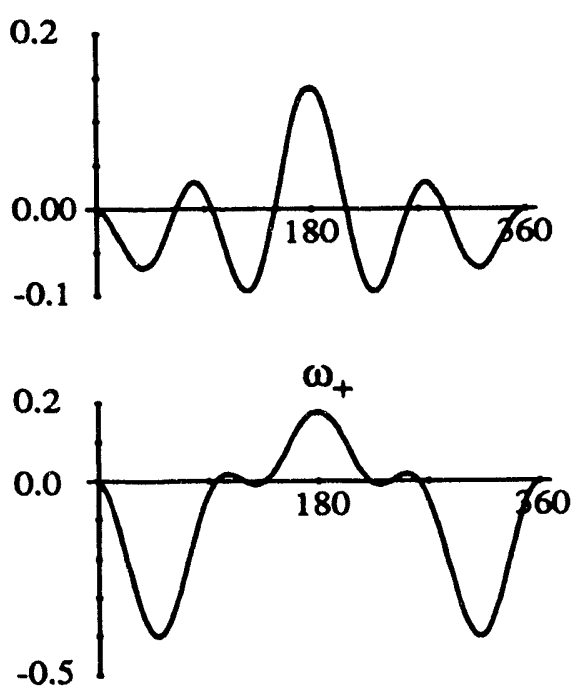

(b)
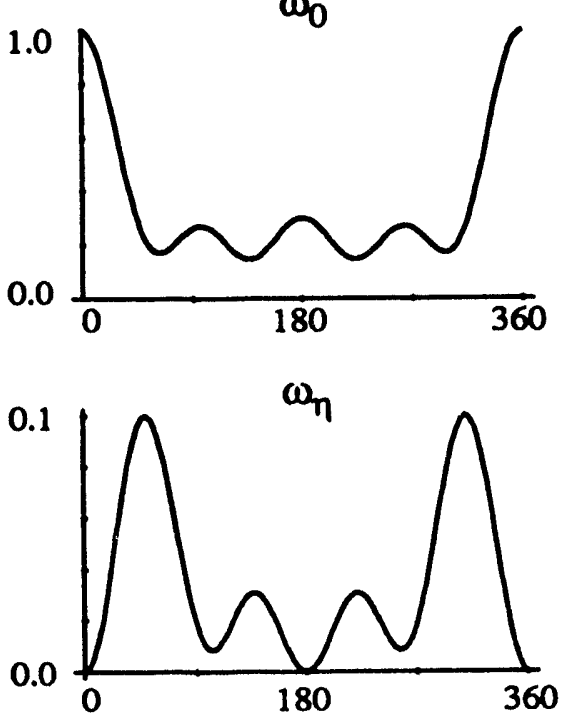

$\omega$
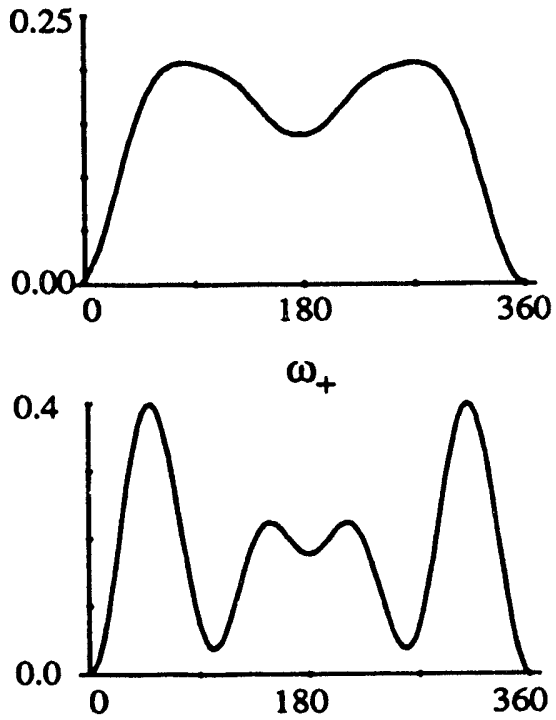

Pulse Length (degree)

Figure 1.2: Pulse responses under the two pulse sequence (see Figure 1.1d) in zero field NMR with different pulse length: (a) $\theta=\theta^{\prime}$, (b) $\theta^{\prime}=\pi-\theta$ or $\theta^{\prime}=-\theta$, where $\omega_{0}$, $\omega_{\eta}, \omega_{-}$, and $\omega_{+}$represent the intensity of transitions corresponding to non-evolved, $\eta$, quadrupolar orders respectively. 
Following the same procedure in deriving Eq.(1.8) yields the powder averaged FID signal at the end of the second evolution period $t_{2}$.

$$
\begin{aligned}
G\left(t_{1}, t_{2}\right) & =\sum_{l_{1}, l_{2}, l_{3}, l_{4}} \sum_{m_{1}, m_{2}, m_{3}, m_{4}, m_{5}, m_{6}} \frac{(-1)^{m_{2}+m_{6}-m_{3}}}{2 l_{4}+1} C_{N}\left(l_{3}, I\right) \\
& \times C\left(l_{1}, l_{2}, l_{4}, m_{1}, m_{5}\right) C\left(l_{2}, l_{3}, l_{4}, m_{4}, m_{1}+m_{5}-m_{4}\right) \\
& \times C\left(l_{1}, l_{2}, l_{4}, m_{2}, m_{6}\right) C\left(l_{2}, l_{3}, l_{4}, m_{3}, m_{2}+m_{6}-m_{3}\right) \\
& \left.\times a_{l_{1}, m_{1}} b_{l_{3}, m_{3}-m_{2}-m_{6}} \mathcal{D}_{m_{5}, m_{4}}^{\left(l_{2}\right)}\left(\Omega_{P}\right) \mathcal{E}_{m_{3}, m_{2}}^{l_{2}, l_{1}}\left(t_{1}\right) \mathcal{E}_{m_{1}+m_{5}-m_{4}, m_{6}}^{l_{3}, t_{2}}\right)
\end{aligned}
$$

After two dimension Fourier transformation, a cross peak appears only when the two diagonal peaks are the different transitions of same site. Experimental results can be seen in reference [11].

\subsection{Zero Field and High Field Correlation Spec- trum}

Time-domain zero field NMR and NQR spectroscopy has provided a new method for obtaining high resolution spectra of a polycrystalline or amorphous sample and therefore allows us to measure the dipolar coupling or quadrupolar coupling constants without the spectral resolution problem [8]-[12]. However, we can observe neither isotropic chemical shifts nor chemical shift anisotropies. This is because the chemical shifts linearly depend on the external magnetic field if they are measured in unit of Hertz. At zero field, the chemical shifts basically are zero. On the other hand, the chemical shift information can be crucial for determining the structure or dynamical behavior of a molecule.

In order to increase the sensitivity of the zero field experiments, a spin-locking technique has been employed[9] for the high field observation. This spin-locking technique effectively disregards the high field spectra and all information contained in it. After a moment more thought, we know that the correlations between zero field and high field are also destroyed by spin-locking detection. H'owever such a 
correlation spectrum[18] will lead to a new type of separation of a usual high field powder pattern based on the high resolution spectrum in the zero field domain and also takes advantage both of zero field and high field after combination with multiple pulse techniques in high field[19, 114]. In this section, we will provide a brief theoretical description of the zero field and high field correlation spectra and show a set of simulations which potentially could be verified by some experiments.

We start with the initial state of the density matrix, $\rho_{0}$, in zero field.

$$
\rho_{0}=\sum_{l, m} a_{l, m} T_{l, m},
$$

where the $T_{l, m}$ are the components of a $l$-th rank irreducible tensor, and the $a_{l, m}$ are the coefficients which represent the initial state of the density matrix prepared by switching off the external magnetic field suddenly, or demagnetization to zero adiabatically and then initialized by application of a strong dc pulse. After we have prepared a suitable initial state in zero field, it is transformed into a molecular frame,

$$
\rho_{0}^{M}=\sum_{l, m, m^{\prime}} a_{l, m} \mathcal{D}_{m^{\prime}, m}^{(l)}\left(\Omega_{M}\right) T_{l, m^{\prime}}
$$

where the $\mathcal{D}_{m^{\prime}, m}^{(l)}$ 's are the components of Wigner rotation matrices, the $\Omega_{M}$ are the Euler angles between the laboratory frame and the molecular frame, and the $T_{l, m^{\prime}}$ 's are the irreducible tensor in the molecular frame. The zero field spin Hamiltonian in the molecular frame may be written in general as

$$
\mathcal{H}_{z}^{M}=\sum_{m}(-1)^{m} A_{2,-m}^{z} T_{2, m},
$$

where the $A_{2, m}^{z}$ 's are the components of an interaction tensor responsible for evolution in zero field (e.g. dipolar, or quadrupolar interaction). With Eq.(1.34), the evolution operator in the molecular frame is

$$
\mathcal{L}_{z}^{M}(t)=e^{-i \mathcal{H}_{z}^{M} t}
$$

After evolution in zero field for time, $t_{1}$, we can use a propagator $P$ to store the coherence parts in the density matrix as a population distribution which relaxes 
slowly. The sample is then moved from zero field to high field for further evolution with high field spin Hamiltonian which is truncated by the large Zeeman interaction, that is,

$$
\mathcal{H}_{h}^{M}=\sum_{m}(-1)^{m} \mathcal{D}_{m, 0}^{(2)}\left(\Omega_{M}\right) A_{2,-m}^{h} T_{2,0}
$$

where $A_{2,-m}^{h}$ is the components of the interaction tensor in high-field (e.g. chemical shift anisotropy). The evolution operator in high-field is then

$$
\mathcal{L}_{h}^{M}(t)=e^{-i \mathcal{H}_{h}^{M} t}
$$

After evolution in high field for a time, $t_{2}$, the total density matrix can be calculated by use of Eqs.(1.33), (1.35), and (1.37).

$$
\rho\left(t_{1}, t_{2}, \Omega_{M}\right)=\mathcal{L}_{h}^{M}\left(t_{2}\right) \mathbf{P} \mathcal{L}_{z}^{M}\left(t_{1}\right) \rho_{0}^{M} \mathcal{L}_{z}^{M-1}\left(t_{1}\right) \mathbf{P}^{-1} \mathcal{L}_{h}^{M-1}\left(t_{2}\right) .
$$

Finally the two-dimensional signal is given by

$$
g\left(t_{1}, t_{2}, \Omega_{M}\right)=\operatorname{Tr}\left\{I_{+} \rho\left(t_{1}, t_{2}, \Omega_{M}\right)\right\}
$$

For a powder sample, an average over $\Omega_{M}$ has to be performed, and then the FID signal is

$$
G\left(t_{1}, t_{2}\right)=\int_{\Omega} g\left(t_{1}, t_{2}, \Omega_{M}\right) d \Omega_{M}
$$

As an illustrative example, we calculated the zero field and high field correlation spectra for a spin system $I=1$ system which evolves under a quadrupolar Hamiltonian in both zero field and high field. The sudden switch pulse scheme is assumed for initial evolution in zero field (see Figure 1.1a). The only difference between the zero field Hamiltonian and high field Hamiltonian is that the Hamiltonian in high-field is the truncated quadrupolar Hamiltonian. In this case we can choose the molecular frame to coincide with the principle axis system of the quadrupolar interaction tensor. The initial state is $I_{z}$ in the laboratory frame. From Eq.(1.33), the initial density matrix in the principle axis system is

$$
\rho_{M}(0)=\sum_{m} \mathcal{D}_{m, 0}^{(1)}\left(\Omega_{M}\right) T_{1, m}
$$


As we know the quadrupolar Hamiltonian in the PAS is

$$
\mathcal{H}_{Q}=\omega_{q}\left[T_{2,0}-\frac{1}{2} \eta\left(T_{2,2}+T_{2,-2}\right)\right]
$$

Under this Hamiltonian, the evolution in the zero field during time $t_{1}$ is completely determined by the evolution matrices $\mathcal{E}^{\left(1, l^{\prime}\right)}$ given in Table (1.3). In the high field domain, the truncated Hamiltonian in the rotating frame is

$$
\mathcal{H}_{Q, 0}=\sum_{m} \mathcal{D}_{m, 0}^{(2)}\left(\Omega_{M}\right) \rho_{2, m} T_{2,0}
$$

By inserting Eqs.(1.41), (1.42), and (1.43) into Eq.(1.38) we can calculate the density matrix for the correlation experiment,

$$
\rho\left(t_{1}, t_{2}, \Omega_{M}\right)=\sum_{l, m_{1}, m_{2}} \mathcal{D}_{m_{1}, 0}^{(1)}\left(\Omega_{M}\right) \mathcal{E}_{m_{2}, m_{1}}^{1, l}\left(t_{1}\right) e^{-\imath \mathcal{H}_{Q, 0} t_{2}} \mathbf{P} T_{l, m_{2}} \mathbf{P}^{-1} e^{\imath \mathcal{H}_{Q, 0} t_{2}}
$$

For the simplest case, the propagator $\mathbf{P}$ first transforms the irreducible tensors from PAS to the laboratory frame, projects it on to $I_{z}$ axis, and finally applies a $90^{\circ}$ to it along $I_{y}$ axis in the rotating frame in high field. Using this definition of $P$ implies

$$
G\left(t_{1}, t_{2}\right)=\int_{\Omega} d \Omega_{M} \sum_{m_{1}, m_{2}} \mathcal{D}_{m_{1}, 0}^{(1)}\left(\Omega_{M}\right) \mathcal{D}_{m_{2}, 0}^{(1)} \dagger\left(\Omega_{M}\right) \mathcal{E}_{m_{2}, m_{1}}^{1,1}\left(t_{1}\right) \cos \left[A_{2,0}\left(\Omega_{M}\right) t_{2}\right] .
$$

Using Eq.(1.45) and Table (1.3), we have performed numerical simulations of the zero field and high field correlation spectra for spin $I=1$ system with quadrupolar interaction . Figure (1.3) shows a variation of powder patterns as the asymmetry parameter, $\eta$, changes from 0 to 1 . The new type of separation based on the high resolution zero field spectra is clearly demonstrated. The complicated powder lineshape in high field domain decomposes into three (two when $\eta=0$ ) relatively simple superimposed lineshapes. Each powder pattern has different shape and corresponds to the different orders (e.g. quadrupolar order, or $\eta$ order) present in the zero field domain. The separation therefore may be useful for assignment of the zero field NQR lines by the superimposed lineshapes, when there are several unequivalent spins in the sample. Such two-dimensional correlation spectra have been measured by Zax and co-workers $[9,11]$. 
(a) $\eta=0.0$

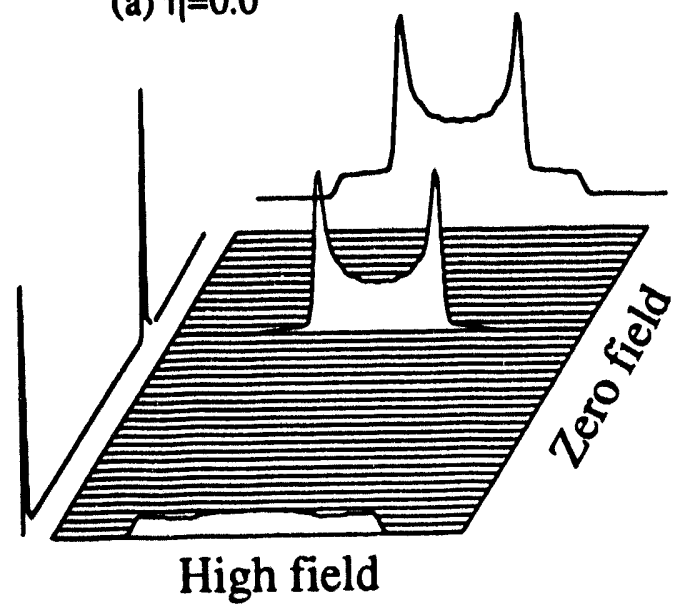

High field

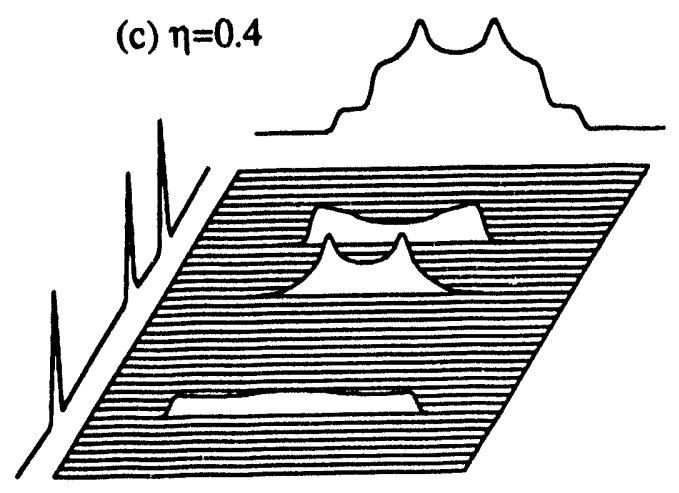

(e) $\eta=0.8$

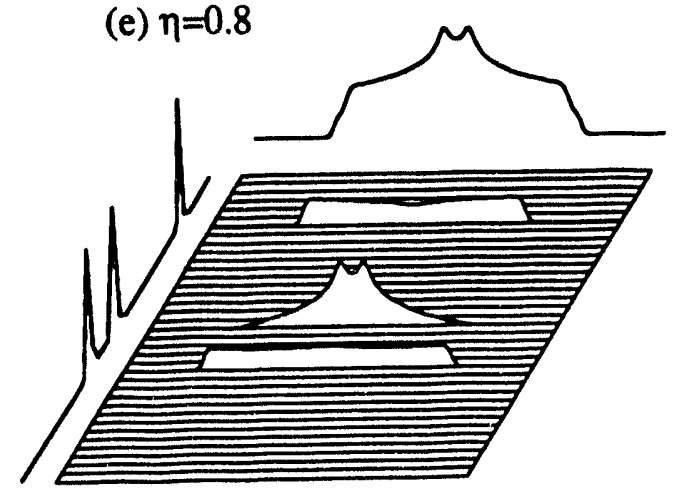

(b) $\eta=0.2$

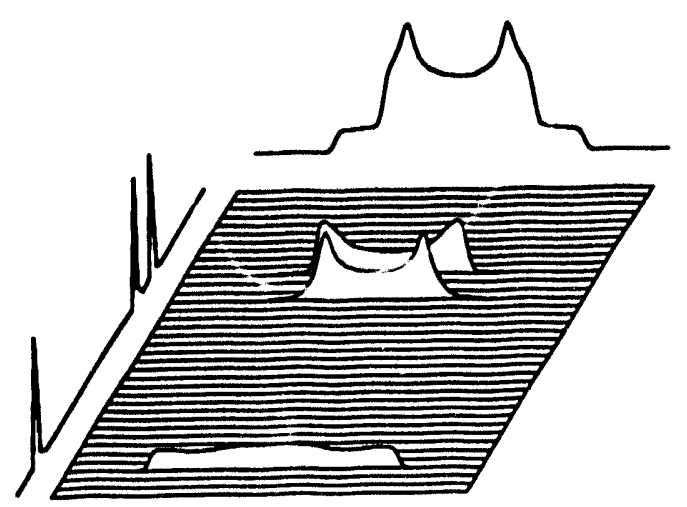

(d) $\eta=0.6$

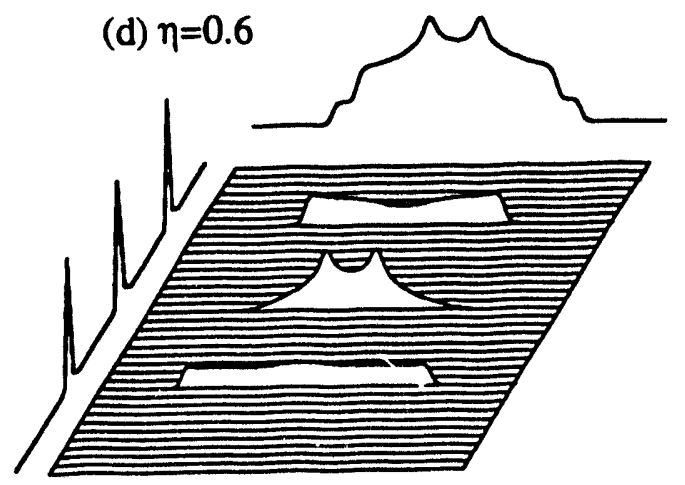

(f) $\eta=1.0$

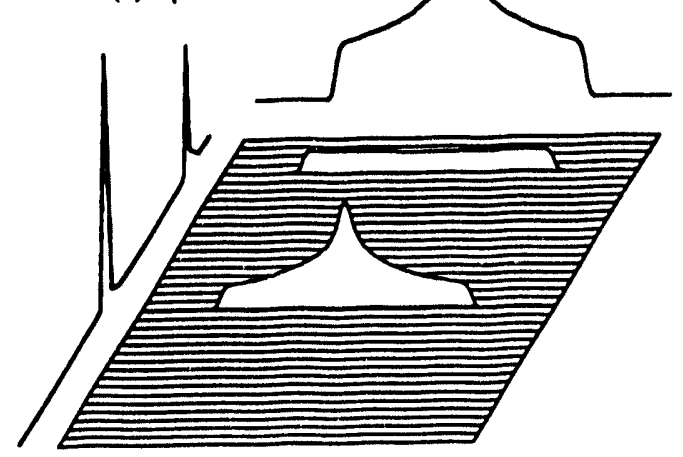

Figure 1.3: Variation of simulated powder lineshapes in zero field and high field correlation spectra with different asymmetry parameter $\eta$ for spin $I=1$ with quadrupolar interactions. The projections of the zero field and high field are shown on the sides of the 2-D spectrum respectively. Each superimposed powder lineshape in high field domain is related to the order in zero field domain 
We can generalize the calculation that resulted in Eq. (1.45) to include other zero field pulse schemes (see Figure $1.1 \mathrm{~b}-\mathrm{d}$ ). The signal as a function of crystal orientation and time in zero field can be shown to be

$$
g_{z}\left(t_{1}, \Omega_{M}\right)=\sum_{l, m_{1}, m_{2}} a_{m_{2}, m_{1}}^{l}\left(t_{1}\right) \mathcal{D}_{m_{2}, m_{1}}^{(l)}\left(\Omega_{M}\right)
$$

where the coefficients, $a_{m_{2}, m_{1}}^{l}\left(t_{1}\right)$, depend on the evolution in zero field, and the summation over $l$ runs from zero to a finite integer number. In other words, the zero field signal can be expressed in a finite number of irreducible representations of $S O$ (3) group. Similar to Eq.(1.46), the high-field signal can also be expressed as

$$
g_{h}\left(t_{2}, \Omega_{M}\right)=\sum_{l, m} b_{m}^{l}\left(t_{2}\right) \mathcal{D}_{m, 0}^{(l)}\left(\Omega_{M}\right)
$$

where the index $l$ now varies from zero to infinity. Two conclusions can be drawn from the above discussion. First, in conventional high-field NMR only the identity representation of $S O(3)$ in the density matrix contributes a powder lineshape. The evolution of the density matrix in the zero field now allows us to observe the high field powder lineshape with different representations of $S O(3)$. In the above example, there are total three representations, $\mathcal{D}^{(0)}, \mathcal{D}^{(1)}$, and $\mathcal{D}^{(2)}$, of $S O(3)$ participated in forming the superimposed lineshapes. Second, the orthogonality of the Wigner rotation matrices implies that the index $m_{2}$ for zero field signal has to be zero after averaging over the powder average. This reduction makes the calculation easier, allowing us to quickly derive the coefficient for Eq. (1.46) for the above example,

$$
a_{m, 0}^{l}\left(t_{1}\right)=\sum_{m_{1}}(-1)^{m} C\left(1,1, l, m_{1}, m-m_{1}\right) C(1,1, l, 0,0) \mathcal{E}_{m_{1}-m_{,} m_{1}}^{1,1}\left(t_{1}\right) .
$$

An interesting application of zero field and high field correlation spectroscopy will be when the evolutions in zero field and high field are governed by different spin Hamiltonians. An example of particular interest is the case of two dipolar coupled spin one half nuclei because in zero field there is only the dipolar interaction of a lone spin pair and in high field there is only the chemical shift anisotropy, assuming 
that the dipolar interaction has been eliminated in high field by applying a multiple pulse sequence (e.g. WAHUHA) [114]. In general, the principle axis systems for these interactions are not the same, so we will introduce a molecular frame which differs from the principle axis systems. For the simplicity, we continue using the same initial density matrix and propagator operator in zero field as before. In order to use Eq.(1.44), we need to add another transformation from the molecular frame to the principle axis system. After this has been done, the zero field signal will have the same form as Eq.(1.46) with the coefficient given by

$$
\begin{aligned}
a_{m, 0}^{l}\left(t_{1}\right) & =\sum_{m_{1}}(-1)^{m} C\left(1,1, l, m_{1}, m-m_{1}\right) C(1,1, l, 0,0) \\
& \times \sum_{m_{3}, m_{4}} \mathcal{D}_{m_{3}, m_{1}}^{(1)}\left(\Omega_{D}\right) \mathcal{D}_{m_{1}-m_{,} m_{4}}^{(1)}{ }^{\dagger}\left(\Omega_{D}\right) \mathcal{E}_{m_{4}, m_{3}}^{1,1}\left(t_{1}\right),
\end{aligned}
$$

where $\Omega_{D}$ is the Euler angles of the transformation from the molecular frame to the principle axis system of the dipolar interaction tensor. For the high-field part of the experiment, the CSA tensor is

$$
A_{2,0}\left(\Omega_{M}, \Omega_{C S}\right)=\sum_{n, n_{1}} \mathcal{D}_{n, 0}^{(2)}\left(\Omega_{M}\right) \mathcal{D}_{n_{1}, n}^{(2)}\left(\Omega_{C S}\right) \rho_{2, n_{1}},
$$

where $\Omega_{C S}$ are the Euler angles of the transformation from the molecular frame to the principle axis system of the chemical shift anisotropy tensor. At the end of the evolution in high field domain, from Eqs.(1.49) and (1.50) the FID signal should be

$$
G\left(t_{1}, t_{2}\right)=\int_{\Omega} d \Omega_{M} \sum_{l, m} a_{m, 0}^{l}\left(t_{1}, \Omega_{D}\right) \mathcal{D}_{m, 0}^{(l)}\left(\Omega_{M}\right) e^{-\imath A_{2,0}\left(\Omega_{M}, \Omega_{C S}\right) t_{2}}
$$

Using Eq.(1.51) and Table (1.3), a variation of powder patterns with different asymmetry parameter $\eta$ and relative orientation between the principle axes of the dipolar coupling tensor and the chemical shift anisotropy tensor is shown in Figure (1.4). In the simulation, we chose the molecular frame to overlap with the principle axis system of the dipolar coupling tensor. The superimposed powder lineshapes corresponding to zero field peaks $\omega_{0}$ and $\omega_{D}$ are strongly dependent on the relative orientation of the chemical shift anisotropy tensor with respect to the molecular frame. 


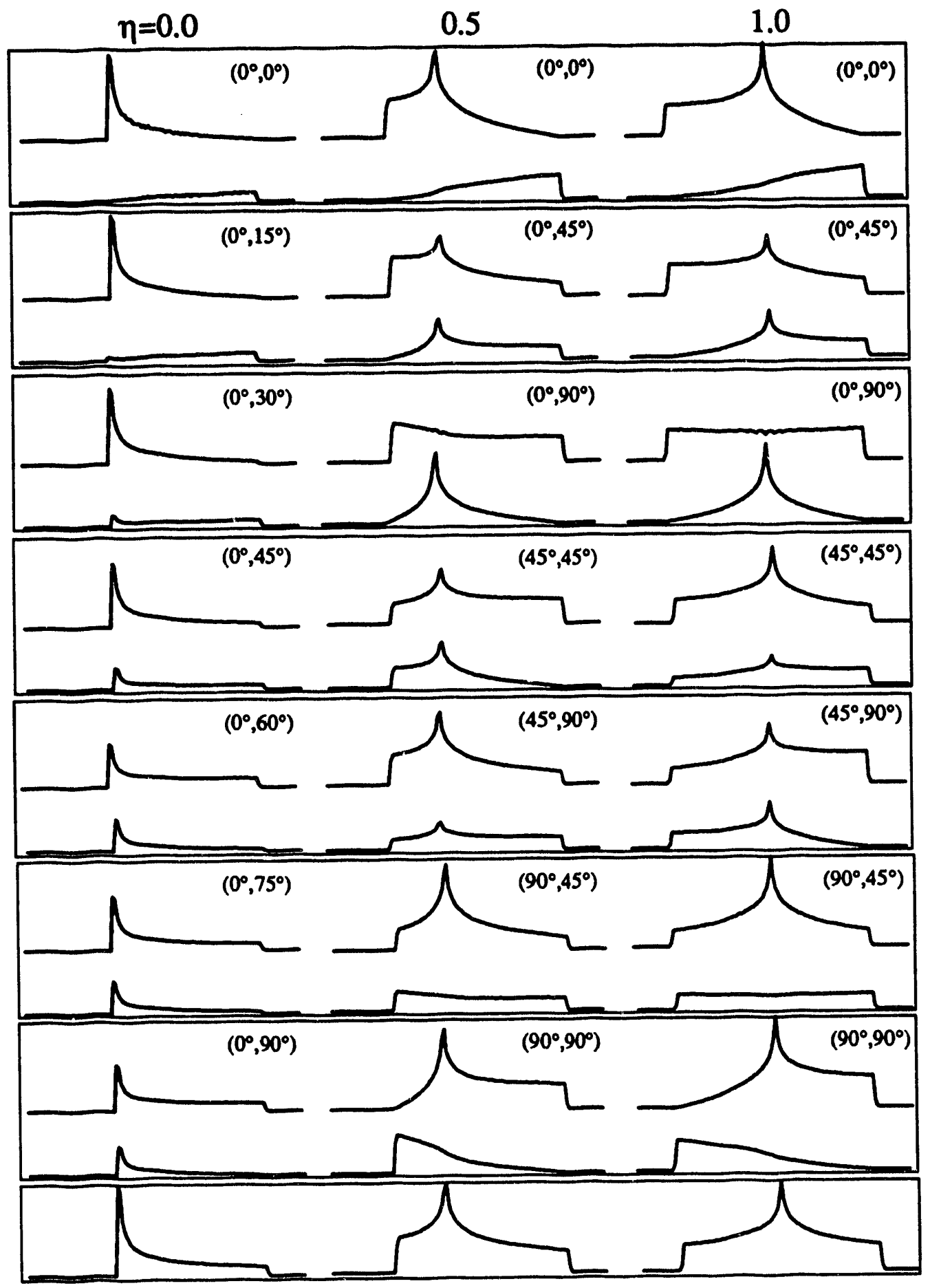

Figure 1.4: Variation of simulated powder lineshapes of dipolar coupling and chemical shift anisotropy correlation spectra with different asymmetry parameter, $\eta$, and the relative orientations between two tensors for two spin $I=\frac{1}{2}$ nuclei with dipolar interaction in zero field and chemical shift anisotropy interaction in high field, assuming that two nuclei are chemical equivalent. $\omega_{0}$ and $\omega_{D}$ are two transition frequencies at zero field. Left: $\eta=0$, middle: $\eta=0.5$, and right: $\eta=1.0$. 
The full information about two interaction tensors, that is, the dipolar coupling constant and the principal values of the chemical shift anisotropy tensor, therefore can be obtained from the zero field and high field correlation spectra. These information will be very important in the determination of a molecule's structure.

Although we have only shown two simple examples in the demonstration of the zero field and high field correlation spectra, other combinations are readily conceivable with different zero field pulse scheme. It has to be pointed out that in order to obtain pure chemical shift anisotropy lineshape for protons, a powerful homonuclear decoupling technique has to be applied in high-field. This will make any real experiments of zero field and high field correlation spectrum very difficult. This is also the reason why we have not shown suitable experimental data in the second example for verification. However, we believe that it can be done in future, and thus this method still is potentially very useful.

\subsection{Spin Diffusion in Zero Field NMR}

Time-domain zero field NMR has proven powerful for obtaining high-resolution spectra of polycrystalline or amorphous samples in solids [8]-[18]. The convenience is obvious in the assignment of the sites, local symmetry, and even the exchange between sites from these spectra. In this section we will examine the spin diffusion (that is the order exchange) process at zero field. It has been shown in high field NMR, that the rate of the spin diffusion relates directly to the distance between two spins as well as the orientations of the internuclear vectors[34]. The measurement of spin diffusion in solids by means of two dimensional spectroscopy in high field NMR $[35,36,37,38,39,40]$ is based on the overlap of lines corresponding to different sites. In the overlap region, the eigenstates of the spin Hamiltonian are mostly degenerate, and therefore, the spins can undergo an energy conserving flip-flop process. Furthermore, the flip-flop transition probability increases as the overlap region in- 
creases. Because the magnetization evolves under a scalar Hamiltonian in zero field, the zero field NMR spectra therefore are highly resolved, leading one to ask whether or not the spin diffusion process is possible. Furthermore, if there is a such process, how can we interpret it? The answer of the first question is positive owing to the experimental observation of the spin diffusion process in zero field [17]. The second question turns out to be much more difficult to answer properly.

In Figure (1.5), two pulse schemes for measuring zero field spin diffusion process are shown: one is the sudden switch scheme (1.5a) and the other is the adiabatic sequence (1.5b). Both schemes are a combination of the pulsed zero field NMR and two-dimensional exchange spectroscopy commonly used in high field NMR[41, 42]. The sample is first polarized in high magnetic field $\left(H_{0}=4.2 T\right)$ and then moved pneumatically to an intermediate field with field strength about $0.01 T$. This field then is suddenly turned off in the first pulse scheme. After the magnetization processes freely for a length of time $t_{1}$ under the spin Hamiltonian, the intermediate field is turned on to store the spin order along the $z$-axis in the laboratory frame. The stored spin order is orientation dependent for each individual spin and, therefore, the spins are not in an equilibrium state. In the $\tau_{m}$ period, the spin system undergoes a diffusion process among different spin orders. After the intermediate field is turned off again, the magnetization evolves for a time $t_{2}$ under same spin Hamiltonian as during the first evolution period, but with the initial state affected by the spin diffusion process which occurred during mixing time. Owing to the existence of the mixed order among different spin nuclei, cross peaks appear in a two-dimensional spectrum and the diffusion rates may be measured from the intensities of the cross peaks (see Figure 1.5c). The diffusion mechanism in this experiment will be same as it in high field[34] and now we will focus on the second pulse scheme, where true zero field spin diffusion occurs.

In the second pulse scheme (Figure 1.5b), the intermediate field is adiabatically 

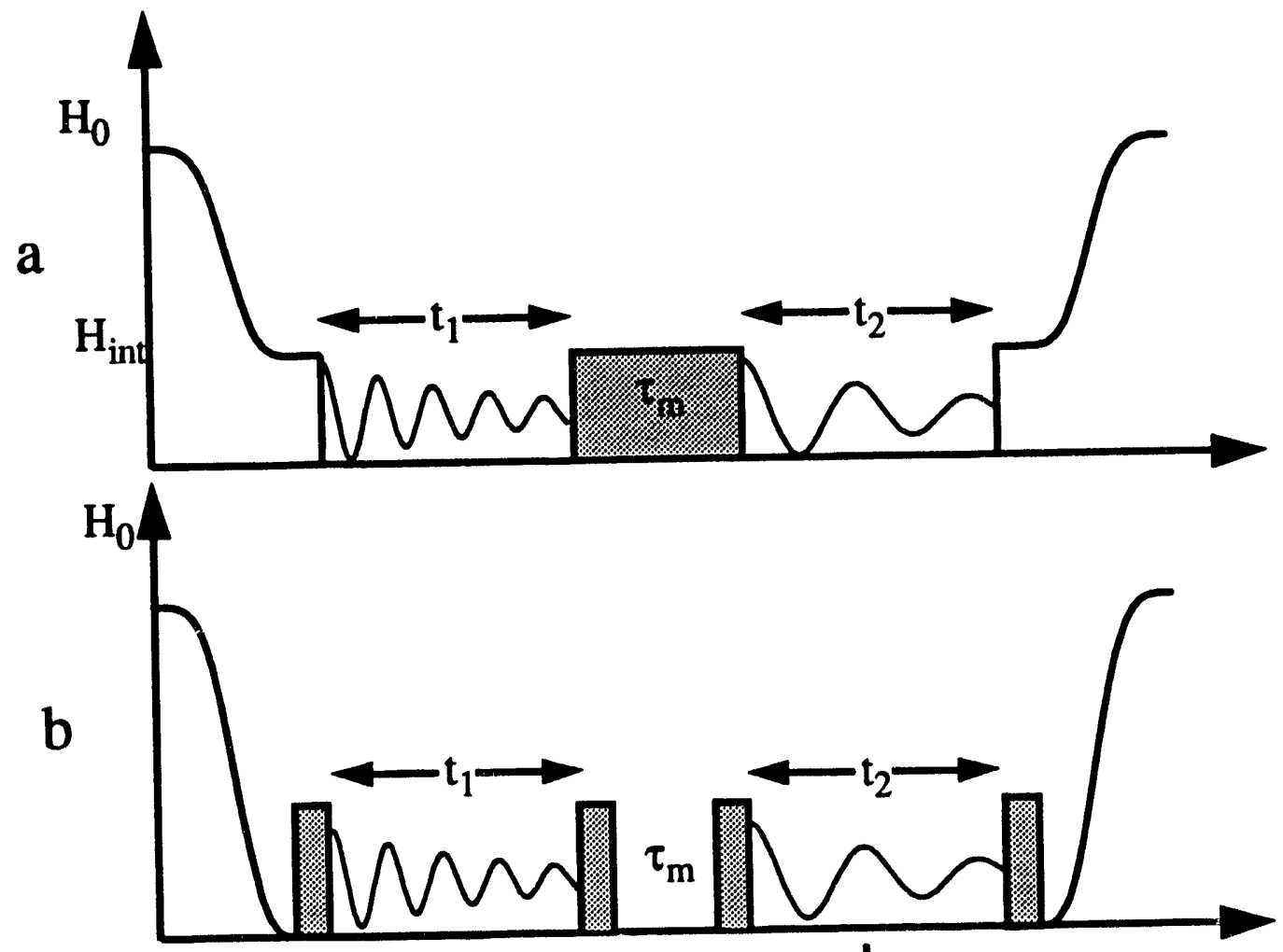

C

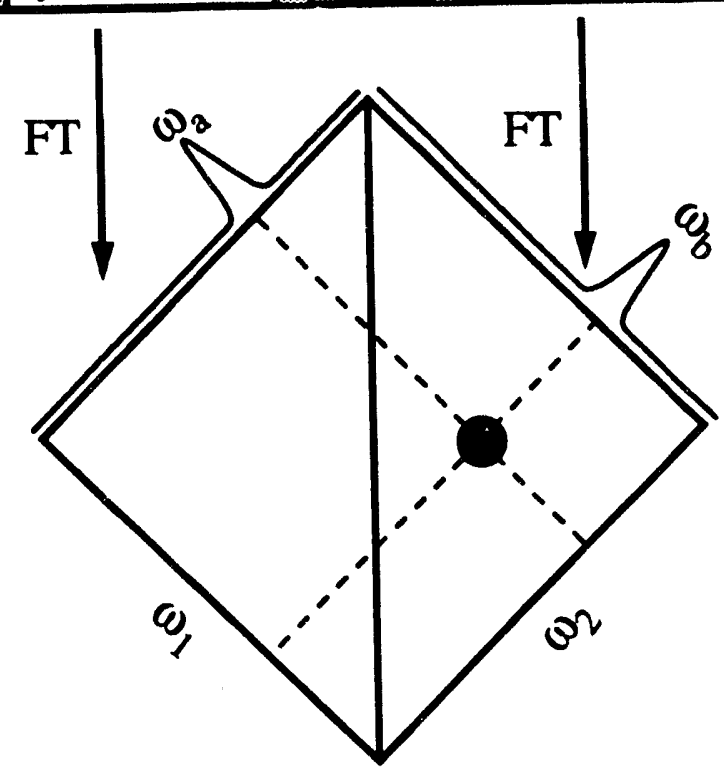

Figure 1.5: Two-dimensional spin diffusion experiment in time-domain zero field NMR used to observe spin diffusion rate. The mixing time $\tau_{m}$ is kept constant in one experiment. Fourier transformation with respect to $t_{1}$ and $t_{2}$ yields a two-dimensional exchange spectrum where the cross peaks indicate the occurrence of the spin diffusion. 


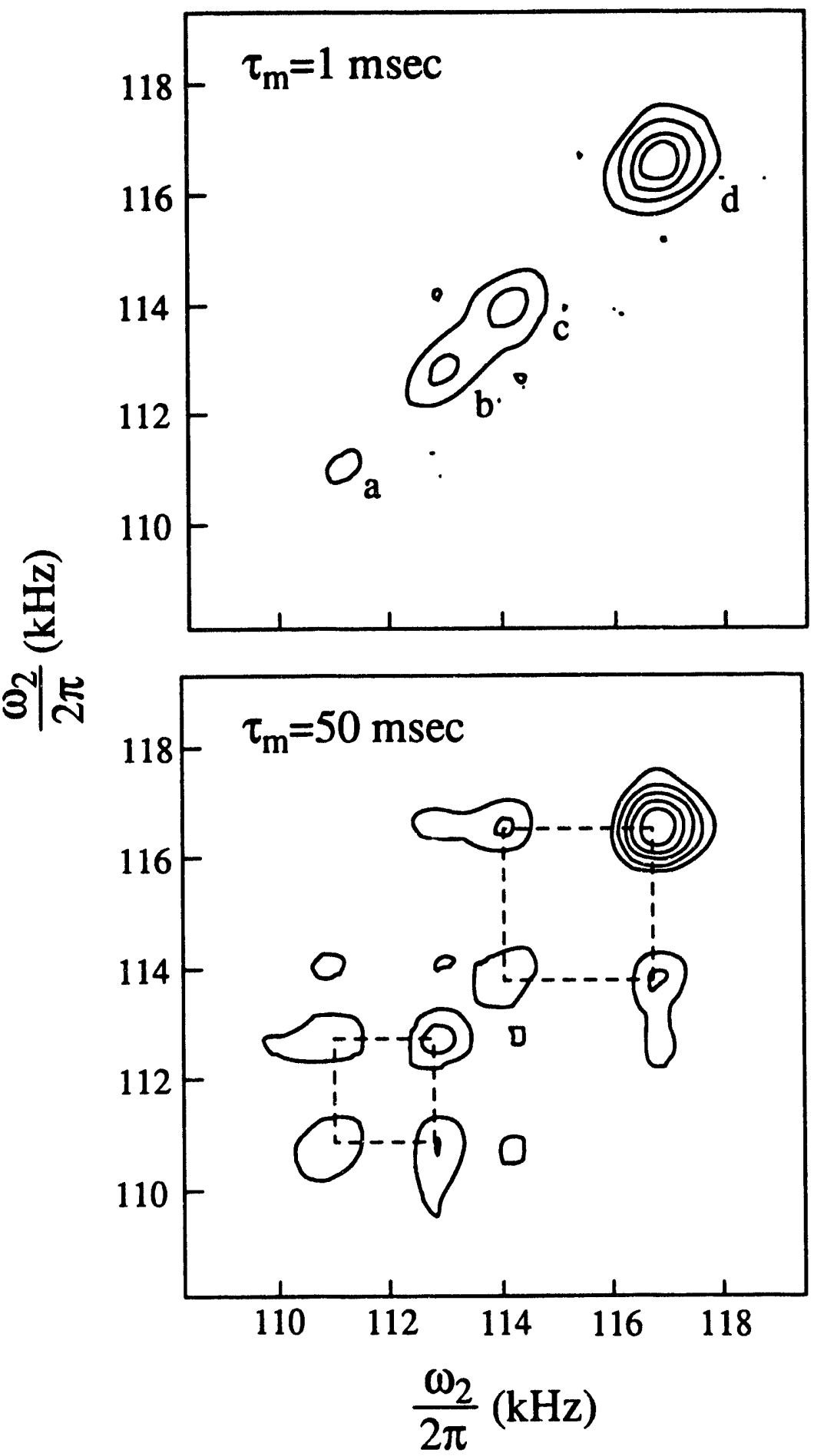

Figure 1.6: Experimental spin diffusion spectra of diethylterephthalate- $d_{4}$. The two-dimensional spectra show only the peaks in the region from $100 \mathrm{kHz}$ to $120 \mathrm{kHz}$ in Figure (1.7). (a) $\tau_{m}=1 \mathrm{msec}$, and (b) $\tau_{m}=50 \mathrm{msec}$. It can be clearly seen that the spin diffusion occurs on a length of time about a few milliseconds. 
reduced to zero. In this process, the Zeeman order of the nuclear spins converts to quadrupolar order (that is the magnetization of the spins is quantized in the local field) during the level crossing[43, 44, 45, 46]. A strong dc pulse is then applied to the spin syscem in order to initiate evolution of the density matrix. After the spin system evolves for time $t_{1}$ under the spin Hamiltonian, a second dc pulse is applied to project the coherence of the density matrix to spin orders along the quadrupolar principle axis. In the next $\tau_{m}$ time, the spins will be subjected to a diffusion process. The populations or spin orders of the individual levels will, therefore, mix with each other bringing the states toward the thermal equilibrium. By introducing the second freeprocession period of a length of time $t_{2}$ generated by the third and fourth dc pulses, the mixed order of the individual levels therefore can be separated and allows us to measure the rate of the spin diffusion from the cross peaks in the two-dimensional spectra by two-dimensional Fourier transformation of the zero field signal $g\left(t_{1}, \tau_{m}, t_{2}\right)$.

Before we dig into the theory of spin diffusion in zero field, we first present the experimental results of diethylterephthalate- $d_{4}$ in Figure (1.6), measured by pulse scheme (1.5b). The anisotropic parameters of the quadrupolar interaction for two different sites on ethyl group are $\omega_{Q_{1}}=114.825 \mathrm{kHz}, \eta_{1}=0.017, \omega_{Q_{2}}=112.35 \mathrm{kHz}$, and $\eta_{2}=0.013$, which are extracted from the zero field spectrum in Figure (1.7). The corresponding energy levels which are dominated by the quadrupolar interactions are shown in Figure (1.8). The states are labeled according to the convention given by Vega[47]. The energy levels indicated by arrows are those connected by the dipolar coupling operators. The kets are given by the product of two sets of eigenvectors of the quadrupolar interactions in the principle axis systems. In general, the spin diffusion only occurs among the energy levels that are connected by the dipolar coupling operators and have the energy differences that are comparable with the amplitude of the dipolar interactions. We therefore expect that spin diffusion only 

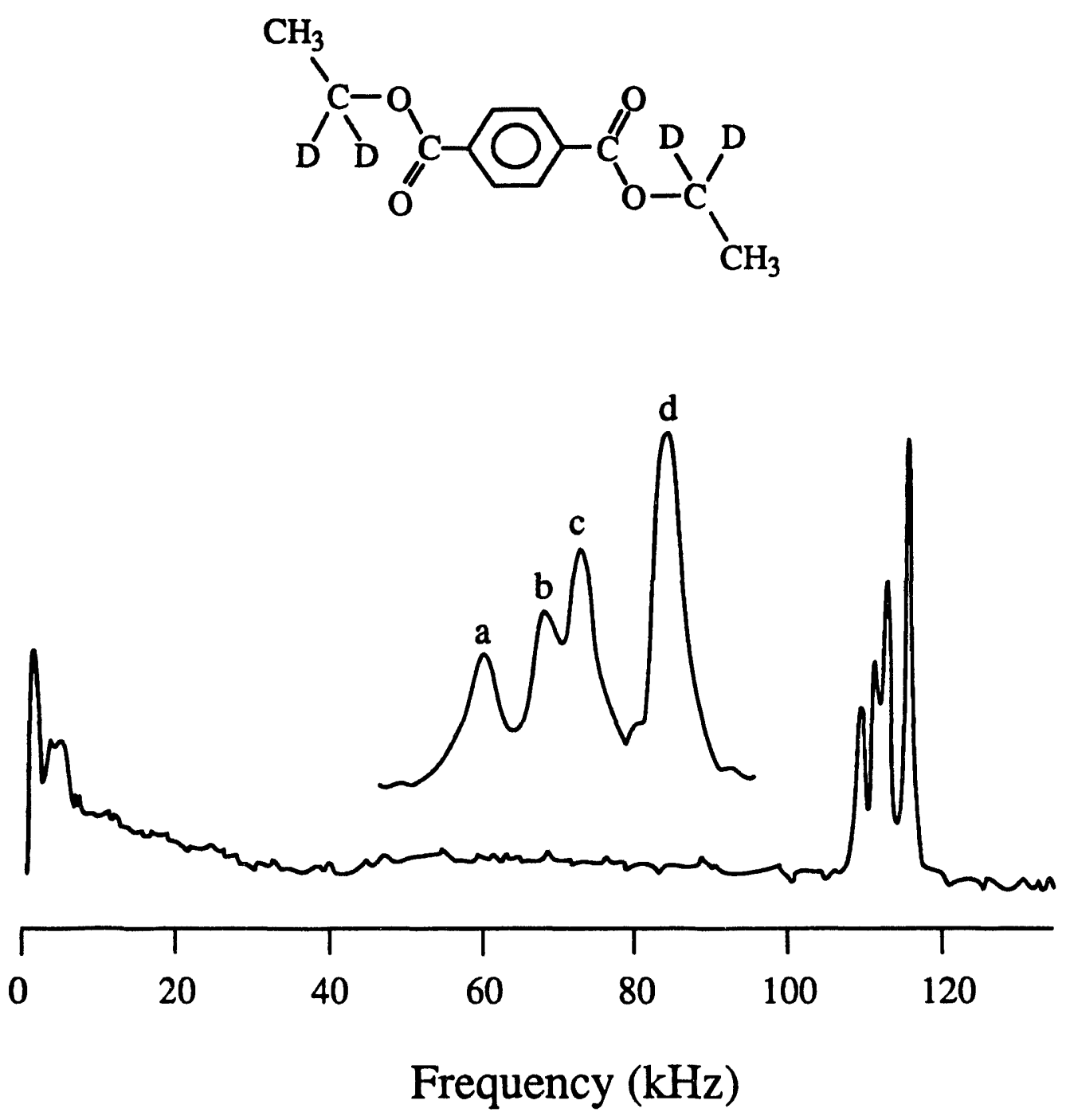

Figure 1.7: Zero field deuterium spectrum of diethylterephthalate- $d_{4}$. The expanded part of the high frequency resonance peaks corresponds to the two-dimensional spectra in Figure (1.6). 
occurs within two subgroups. Each subgroup consists of four energy levels. From Figure (1.6), the experimental results show no cross peaks for short mixing time between different sites. At longer times of several milliseconds, cross peaks between $\mathrm{a}$ and $\mathrm{b}$ and between $\mathrm{c}$ and $\mathrm{d}$ which belong to different sites appear, indicating the occurrence of spin diffusion.

The spin-diffusion process which occurs during the mixing time involves magnetic dipolar interactions between spins. The strength of the dipolar interaction is defined by the coupling constant which is proportional to the gyromagnetic ratios $\gamma_{1}$ and $\gamma_{2}$ of two coupled spins and inverse to the distance between two spins, that is

$$
\omega_{D}=\frac{\gamma_{1} \gamma_{2} \hbar^{2}}{r_{1,2}^{3}} .
$$

Obviously the rate of the spin diffusion therefore is dependent on the internuclear distance.

For the two coupled deuterons, the total Hamiltonian of the system can be written as

$$
\mathcal{H}=\mathcal{H}_{Q_{1}}+\mathcal{H}_{Q_{2}}+\mathcal{H}_{D}
$$

where, in the molecular frame, the explicit expressions of individual terms are

$$
\begin{aligned}
& \mathcal{H}_{Q_{i}}=\sum_{m^{\prime}, m} \mathcal{D}_{m^{\prime}, m}^{(2)}\left(\Omega_{P_{i}}\right) \rho_{2, m^{\prime}}^{i} T_{2, m}^{i}, \\
& \mathcal{H}_{D}=\sum_{m^{\prime}, m} \mathcal{D}_{m^{\prime}, m}^{(2)}\left(\Omega_{P_{1,2}}\right) \rho_{2, m^{\prime}}^{1,2} T_{2, m}^{1,2},
\end{aligned}
$$

and $\Omega_{P_{i}}$ are the Euler angles of the transformation from molecular frame to the principle axis system of the $i$-th interaction. The zero order eigenstates which correspond to the case that the dipolar coupling constant is zero is just the product of the two set of eigenstates of the individual spins, and the unitary transformation operator to the eigen basis is the product of the two rotation operator, that is, $\mathbf{U}=\mathbf{R}_{1}\left(\Omega_{P_{1}}\right) \mathbf{R}_{2}\left(\Omega_{P_{2}}\right)$ where these two rotation operators commute with each other.

Because the dipolar interaction is usually very small for deuterons, during free precession, we can neglect the dipolar interaction, and the density matrix will evolve 

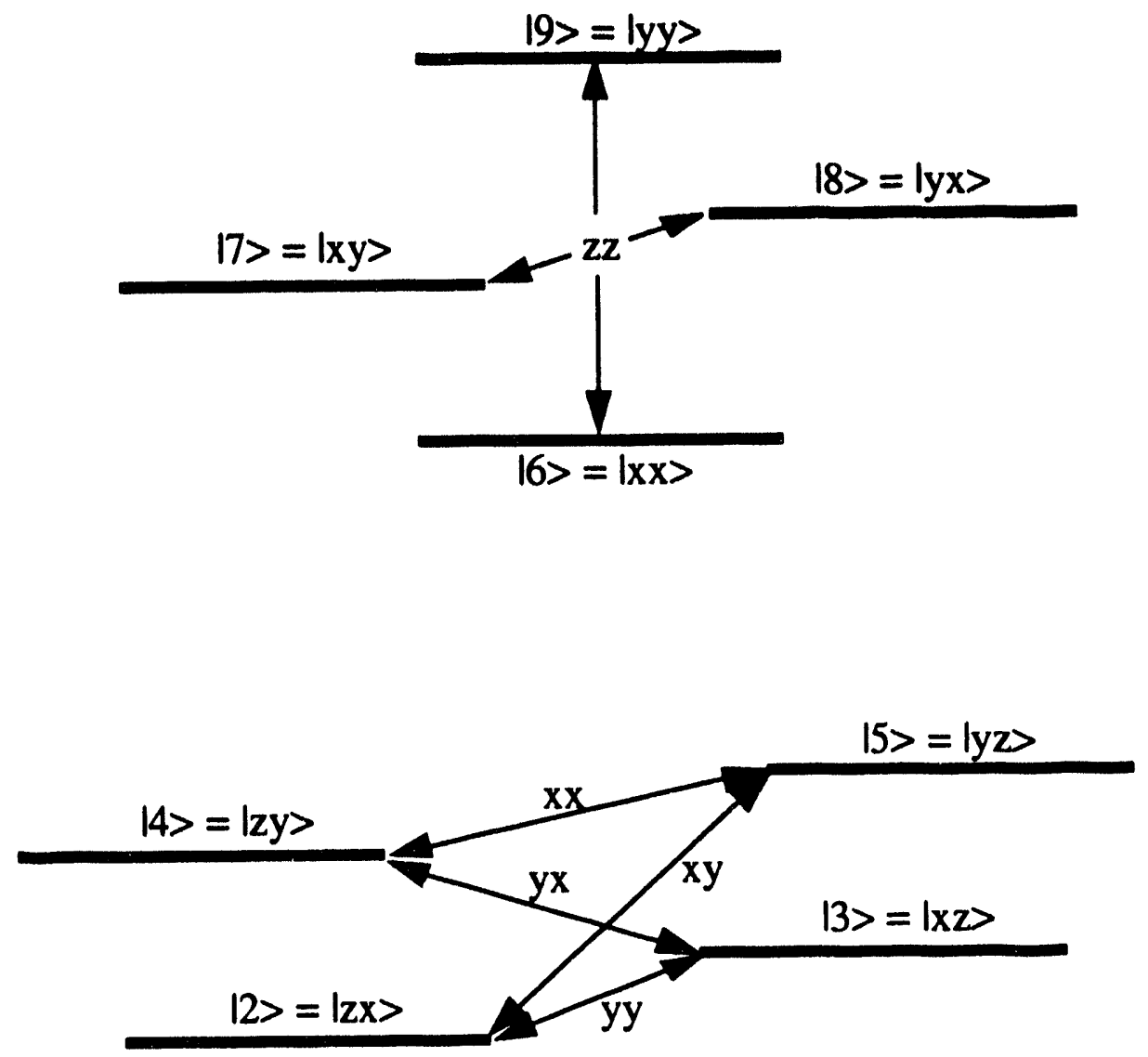

$11\rangle=|z z\rangle$

Figure 1.8: Schematic energy levels for a two coupled spin system with $I=1$ in zero field. The two sets of the principle parameters of quadrupolar interactions are of similar magnitude. The energy levels indicated by the arrows are connected by the elements of the dipolar coupling and do not commute with the main quadrupolar Hamiltonian. 
primarily because of the quadiupolar interactions. After application of the second dc pulse, the density matrix in th.e molecule frame then can be written as

$$
\rho_{M}\left(t_{1}\right)=\sum_{i, l, n} a_{l, n}^{i}\left(t_{1}\right) T_{l, n}^{i}
$$

where terms which do not commute with the main spin Hamiltonian should be dropped because the spin diffusion is much slower than the spin-spin relaxation during the mixing time.

In order to explicitly relate the rate of the spin diffusion with the coupling constant, we transfer the Liouville-von Neumann equation (with $\hbar=1$ )

$$
\frac{d}{d t} \rho_{M}(t)=-2\left[\mathcal{H}, \rho_{M}(t)\right]
$$

into the interaction picture with respect to the zero order Hamiltonian $\mathcal{H}_{Q_{1}}+\mathcal{H}_{Q_{2}}$, defined by

$$
\rho_{M}^{T}(t)=e^{\imath\left(\mathcal{H}_{Q_{1}}+\mathcal{H}_{Q_{2}}\right) t} \rho_{M}(t) e^{-\mathfrak{z}\left(\mathcal{H}_{Q_{1}}+\mathcal{H}_{Q_{2}}\right) t},
$$

during the mixing time. In the interaction picture, the dipolar Hamiltonian becomes time dependent, and the density matrix is determined by

$$
\frac{d}{d t} \rho_{M}^{T}(t)=-\imath\left[\mathcal{H}_{D}^{T}(t), \rho_{M}^{T}(t)\right]
$$

The solution of the Eq.(1.58) can be obtained by use of the time dependent perturbation theory, or by iteration which leads to a power-series expansion with respect to the dipolar Hamiltonian:

$$
\frac{d}{d t} \rho_{M}^{T}(t)=-\imath\left[\mathcal{H}_{D}^{T}(t), \rho_{M}^{T}(0)\right]-\imath \int_{0}^{t} d \tau\left[\mathcal{H}_{D}^{T}(t),\left[\mathcal{H}_{D}^{T}(t-\tau), \rho_{M}^{T}(0)\right]\right]+\cdots
$$

Since the density matrix can be completely represented by a set of irreducible tensors of the spin system, the density matrix in the interaction picture also has the form

$$
\rho_{M}^{T}(t)=\sum_{l, m, p} a_{l, m, p}^{T}(t) T_{l, m}(p)
$$


where $p=l_{i} l_{j}$ for all permutations of spins, $i, j=1,2$. Inserting Eq.(1.60) into Eq.(1.59) leads an equation of motion for the coefficients by multiplying both sides with $T_{l, m}$ and using the orthogonal condition of irreducible tensors (see Eq. 1.9).

$$
\begin{aligned}
& \frac{d}{d t} a_{l, m, p}^{T}(t)=\frac{1}{C_{N}(l, I)}\left[\sum_{l^{\prime}, m^{\prime}, p^{\prime}}-2 a_{l^{\prime}, m^{\prime}, p^{\prime}}^{T}(0) \operatorname{Tr}\left\{\left[\mathcal{H}_{D}^{T}(t), T_{l^{\prime}, m^{\prime}}\left(p^{\prime}\right)\right] T_{l, m}(p)\right\}\right. \\
& \left.+\sum_{l^{\prime}, m^{\prime}, p^{\prime}} a_{l^{\prime}, m^{\prime}, p^{\prime}}^{T}(0) \int_{0}^{t} d \tau \operatorname{Tr}\left\{\left[\mathcal{H}_{D}^{T}(t), T_{l, m}(p)\right]\left[\mathcal{H}_{D}^{T}(t-\tau), T_{l^{\prime}, m^{\prime}}\left(p^{\prime}\right)\right]\right\}\right],
\end{aligned}
$$

where $C(l, I)$ is the normalization constant given by Eq. (1.8).

Similar to the treatment of the density matrix, the dipolar Hamiltonian can also be represented by irreducible tensors

$$
\mathcal{H}_{D}^{T}(t)=\sum_{l, m, p} b_{l, m, p}^{T}(t) T_{l, m}(p) .
$$

The application of the perturbation theory allows us to extend the integration limit to infinity, and to replace the $a_{l, m}^{T}(0)$ by $a_{l, m}^{T}(t)$ based on the fact that the density matrix relaxes very slowly with respect to the evolution frequency. Inserting Eq.(1.62) into Eq.(1.61) yields the evolution equation for the coefficients.

$$
\frac{d}{d t} a_{l, m, p}^{T}(t)=\sum_{l^{\prime}, m^{\prime}, p^{\prime}} W_{m^{\prime}, m, p, p^{\prime}}^{l^{\prime}, l} a_{l^{\prime}, m^{\prime}, p^{\prime}}^{T}(t)
$$

where the rate constants $W_{m^{\prime}, m}^{l^{\prime}, l}$ are given by

$$
\begin{aligned}
& W_{m^{\prime}, m_{1}, p^{\prime}, p}^{l^{\prime}}=\frac{1}{C_{N}(l, I)} \sum_{l_{1}, m_{1}, p_{1}}\left[-\imath b_{l_{1}, m_{1}, p_{1}}^{T}(t) \operatorname{Tr}\left\{\left[T_{l_{1}, m_{1}}\left(p_{1}\right), T_{l^{\prime}, m^{\prime}}\left(p^{\prime}\right)\right] T_{l, m}(p)\right\}\right. \\
& +\sum_{l_{2}, m_{2}, p_{2}} \int_{0}^{\infty} d \tau b_{l_{1}, m_{1}, p_{1}}^{T}(t) b_{l_{2}, m_{2}, p_{2}}^{T}(t-\tau) \\
& \left.\times \operatorname{Tr}\left\{\left[T_{l_{1}, m_{1}}\left(p_{1}\right), T_{l_{1, m}}(p)\right]\left[T_{l_{2}, m_{2}}\left(p_{2}\right), T_{l^{\prime}, m^{\prime}}\left(p^{\prime}\right)\right]\right\}\right] .
\end{aligned}
$$

This formula is in principle same as that given by Suter et al.[34] except we write it in forms of irreducible tensors. A further difference is that Eq.(1.64) is derived in the molecular frame and therefore it is independent of the orientation of the molecular 
frame with respect to the laboratory frame. The rates of spin diffusion only depend on the relative orientations among the molecular frame and the principle axis systems of quadrupolar interactions and dipolar interactions, which are determined by the molecular structure. In other words, the spin diffusion rates measured in zero field with a powder sample are same as those in high field with single crystal. This is the most important advantage to measure spin diffusion rates in zero field. We must indicate that the time evolution determined by Eq.(1.63) is still reversible, and to make it irreversible we have to restrict the irreducible tensor set $\left\{T_{l, m}\right\}$ to a few relevant observables of the system and set $a_{l, m}^{T}(t)=0$ for all other irrelevant irreducible ten $\mathrm{rs}$. This procedure assumes that disregarded degrees of freedoms are at infinite tempe:-ature, irrespective of the transfer order. This assumption differs from semi-classicas elaxation theory $[48,6]$.

The first term in Eq.(1.64) determines the precession frequency in the double principle axis systems. According to our assumption, only the spin orders in the density matrix participate in spin diffusion and therefore the first term will be zero. We now can see that the spin diffusion rates in zero feld are also proportional to the square of the dipolar coupling constants.

For two coupled deuterons, there are sixty-four components of the coupled irreducible tensors from ranks zero to four, and seventeen additional uncoupled irreducible tensors from rank zero to two. All the components of the coupled irreducible tensors are listed in the Tables (1.5) and (1.6). Thus, the coefficients $\left\{a_{l, m}^{T}(t)\right\}$ will form an eighty-one dimensional vector, and the formal solution of Eq.(1.63) can be written as

$$
\mathbf{A}^{T}(t)=e^{\hat{\mathbf{W}} t} \mathbf{A}^{T}(0),
$$

where $\mathbf{A}^{T}(t)=\left[a_{0,0}^{T}(t), \cdots, a_{4,4}^{T}(t)\right]$. Because the rate matrix $\hat{\mathbf{W}}$ is independent of the orientation of the molecular frame with respect to the laboratory frame, the diagonalization has to be performed only once. The orientation dependence will 
be included in the initial density matrix, $A^{T}(0)$. Because we neglect the dipolar Hamiltonian during the free procession of the magnetization in the first evolution period, there are only uncoupled orders in the density matrix at the beginning of spin diffusion, and the explicit form of the coefficients $\mathbf{A}^{T}(0)$ as functions of the time and the orientation of the molecule frame with respect to the laboratory frame can easily be calculated by use of the method of section 1.3. It is possible that the powder average be analytically solved at the end of the whole evolution. This property will possibly allow us to numerically simulate the spin-diffusion process.

We now calculate the initial coefficients of $\mathbf{A}^{T}(0)$. From the experimental pulse scheme (see Figure 1.5b), and assuming that the adiabatic demagnetization is perfect, the initial density matrix in the molecular frame is

$$
\rho_{M}(0)=\sum_{m^{\prime}, m}\left[\mathcal{D}_{m^{\prime}, m}^{(2)}\left(\Omega_{P_{1}}\right) a_{2, m}^{1}(0) T_{2, m^{\prime}}^{1}+\mathcal{D}_{m^{\prime}, m}^{(2)}\left(\Omega_{P_{2}}\right) a_{2, m}^{2}(0) T_{2, m^{\prime}}^{2}\right]
$$

where

$$
a_{2,0}^{i}=\sqrt{\frac{3}{2}}, a_{2, \pm 1}^{i}=0, \quad a_{2, \pm 2}^{i}=\frac{1}{2},
$$

for $i=1,2$. After the application of the first pulse, the density matrix becomes

$$
\begin{aligned}
\rho_{M}\left(0_{+}\right) & =\sum_{m^{\prime}, m_{m_{1}, m_{2}}} \mathcal{D}_{m^{\prime}, m_{1}}^{(2)}{ }^{\dagger}\left(\Omega_{M}\right) e^{-\imath m_{1} \theta} \mathcal{D}_{m_{2}, m_{1}}^{(2)}\left(\Omega_{M}\right) \\
& \times\left[\mathcal{D}_{m^{\prime}, m}^{(2)}\left(\Omega_{P_{1}}\right) a_{2, m}^{1}(0) T_{2, m_{2}}^{1}+\mathcal{D}_{m^{\prime}, m}^{(2)}\left(\Omega_{P_{2}}\right) a_{2, m}^{2}(0) T_{2, m_{2}}^{2}\right]
\end{aligned}
$$

assuming that the dc pulse is applied along the $z$ axis in the laboratory frame. Now the spin system evolves for a length of time $t_{1}$, and the density matrix is

$$
\begin{aligned}
\rho_{M}\left(t_{1}\right) & =\sum_{l_{1}, m^{\prime}, m_{1}, m_{1}, m_{2}, m_{3}} \mathcal{D}_{m^{\prime}, m_{1}}^{(2)} \dagger^{\dagger}\left(\Omega_{M}\right) e^{-\imath m_{1} \theta} \mathcal{D}_{m_{2}, m_{1}}^{(2)}\left(\Omega_{M}\right) \\
& \times \sum_{i=1,2} \mathcal{D}_{m^{\prime}, m}^{(2)}\left(\Omega_{P_{i}}\right) a_{2, m}^{i}(0) \mathcal{E}_{m_{3}, m_{2}}^{l_{1}, 2}\left(t_{1}, i\right) T_{l_{1}, m_{3}}^{i} .
\end{aligned}
$$

Finally applying the second dc pulse yields

$$
\rho_{M}\left(t_{1}\right)=\sum_{i, l, n} a_{l, n}^{i}\left(t_{1}\right) T_{l, n}^{i}
$$


where

$$
\begin{aligned}
a_{l, n}^{i}(t) & =\sum_{m^{\prime}, m_{,}, m_{1}, m_{2}, m_{3}, m_{4}} \mathcal{D}_{m^{\prime}, m_{1}}^{(2)}{ }^{\dagger}\left(\Omega_{M}\right) \mathcal{D}_{m_{2}, m_{1}}^{(2)}\left(\Omega_{M}\right) \mathcal{D}_{m_{3}, m_{4}}^{(l)} \dagger^{\dagger}\left(\Omega_{M}\right) \\
& \times \mathcal{D}_{n, m_{4}}^{(l)}\left(\Omega_{M}\right) e^{-i\left(m_{1}+m_{4}\right) \theta} \mathcal{D}_{m^{\prime}, m^{\prime}}^{(2)}\left(\Omega_{P_{i}}\right) \rho_{2, m}^{i} \mathcal{E}_{m_{3}, m_{2}}^{l, 2}(t, i),
\end{aligned}
$$

and $i=1,2$ is the spin index.

The next step is to evaluate the coefficients in Eq.(1.62) of the dipolar Hamiltonian in the interaction picture. From Eq.(1.54) and Table (1.5), we are able to rewrite the dipolar Hamiltonian in the molecular frame as

$$
\mathcal{H}_{D}=\sum_{m, m^{\prime}} \omega_{D} \mathcal{D}_{0, m}^{(2)}\left(\Omega_{P_{1,2}}\right) C\left(112, m^{\prime}, m-m^{\prime}\right) T_{1, m^{\prime}}^{1} T_{1, m-m^{\prime}}^{2},
$$

where the $C\left(112, m^{\prime}, m-m^{\prime}\right)$ are the Clebsch-Gordan coefficients. This Hamiltonian is transferred from the molecular frame to the double principle axis systems of the two quadrupolar interactions by the unitary transformation $\mathbf{U}=\mathbf{R}_{1}\left(\Omega_{P_{1}}\right) \mathbf{R}_{2}\left(\Omega_{P_{2}}\right)$, and then transferred into the interaction picture by use of the unitary transformation $e^{-\mathfrak{z}\left(\mathcal{H}_{Q_{1}}+\mathcal{H}_{Q_{2}}\right) t}$, and finally, transferred back to the molecular frame again.

$$
\mathcal{H}_{D}^{T}(t)=\sum_{l, n} b_{l, n}^{T}(t) T_{l, n}\left(l_{1}, l_{2}\right)
$$

with

$$
\begin{aligned}
b_{l, n}^{T}(t) & =\sum_{l_{1}, l_{2}, m, m^{\prime}, m_{1}, m_{2}, m_{3}} \omega_{D} \mathcal{D}_{0, m}^{(2)}\left(\Omega_{P_{1,2}}\right) \mathcal{D}_{m_{1}, m^{\prime}}^{(1)}\left(\Omega_{P_{1}}\right) \mathcal{D}_{m_{2}, m-m^{\prime}}^{(1)}\left(\Omega_{P_{2}}\right) \\
& \times V_{m^{\prime}, m-m^{\prime}} \mathcal{E}_{m_{3}, m_{1}}^{l_{1,1}}(t, 1) \mathcal{E}_{n-m_{3}, m_{2}}^{l_{2,1}}(t, 2) C\left(l_{1}, l_{2}, l, m_{3}, n-m_{3}\right)
\end{aligned}
$$

The matrix representation of the dipolar Hamiltonian in the double principle axis systems has the form in Figure (1.9a). There are thirty-six elements that are not zero and no non-zero diagonal terms. Figure (1.9b) shows the matrix elements of the flip-flop term in the Hamiltonian which is the origin of spin diffusion in high field. According to our discussion at the beginning of this section, there are only 

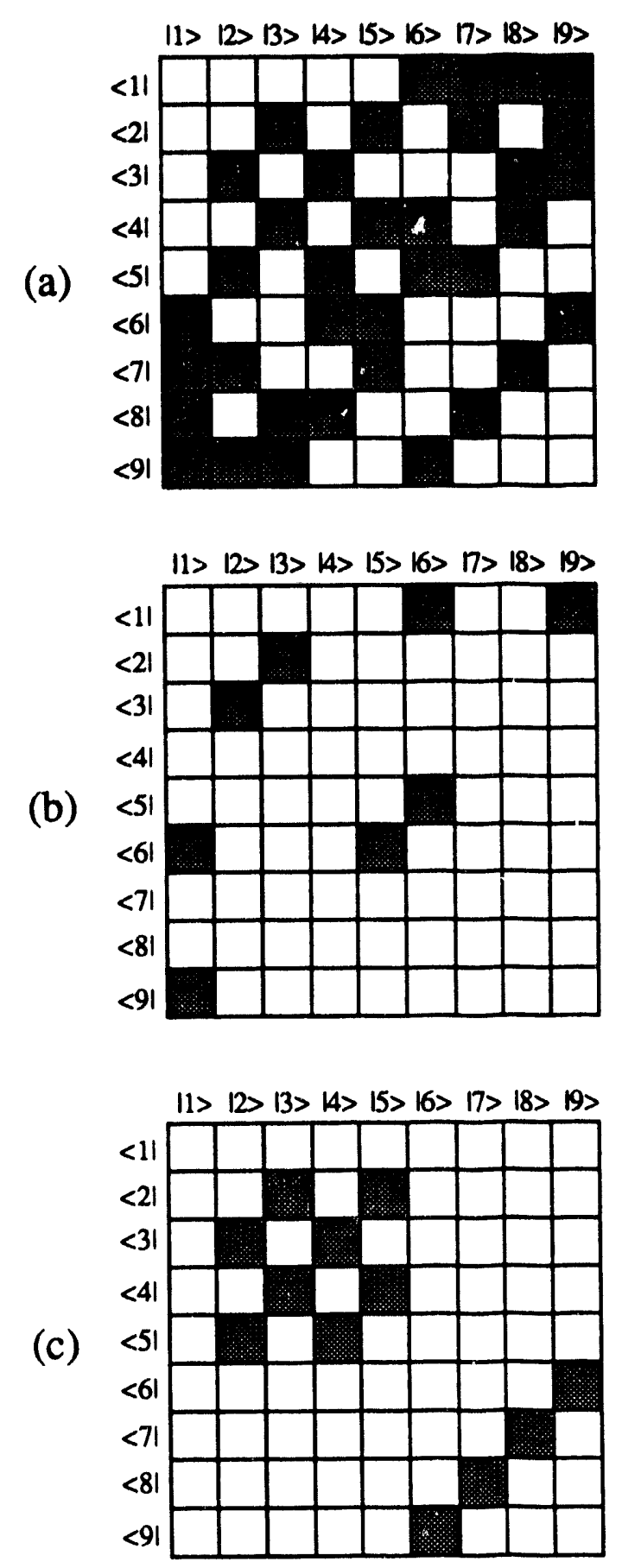

Figure 1.9: Schematic matrix representation of the dipolar Hamiltonian in the double principle axis systems of two quadrupolar interactions for two coupled spins with $I=1$ in zero field. The shaded blocks correspond to non-zero elements in the matrix of the dipolar Hamiltonian, which may have the contribution to the spin diffusion: (a) representation of the whole dipolar Hamiltonian, (b) representation of the flip-flop term, and (c) representation of the matrix which actually contributes to spin diffusion. 
two subgroups in which spin diffusion can possibly occur with total six different rate parameters. These six rate parameters are related to the matrix elements of the dipolar Hamiltonian in Figure (1.9c) and their explicit expression may be calculated from Eq.(1.74). Once this is done, the rate matrix $\hat{W}$ can be obtained by use of Eq.(1.64). The new spin order distribution may be obtained from Eq.(1.65) and the density matrix at the end of the mixing time should have same form as Eq.(1.70). After the magnetization evolves for another length of time $t_{2}$, the FID signal is

$$
\begin{aligned}
& g\left(t_{1}, t_{2}, \tau_{m}\right)=\sum_{i, l, n_{n}, m_{1}, \cdots, m_{6}} \mathcal{D}_{m_{1}, m_{2}}^{(l)}{ }^{\dagger}\left(\Omega_{M}\right) \mathcal{D}_{m_{3}, m_{2}}^{(l)}\left(\Omega_{M}\right) \mathcal{D}_{m_{4}, m_{5}}^{(2)}{ }^{\dagger}\left(\Omega_{M}\right) \\
& \times \mathcal{D}_{m_{6}, m_{5}}^{(2)}\left(\Omega_{M}\right) e^{-i\left(m_{2}-m_{5}\right) \theta} \mathcal{D}_{n_{,} m_{6}}^{(2)}\left(\Omega_{P_{i}}\right) a_{2,-n}^{i}(0) a_{2, m_{1}}^{i}\left(t_{1}, \tau_{m}\right) \mathcal{E}_{m_{4}, m_{3}}^{2, l}\left(i_{2}, i\right),
\end{aligned}
$$

After two-dimensional Fourier transformation of Eq.(1.75), the intensity of a cross peak created during spin diffusion may be calculated and the spin diffusion rate can therefore be deiermined.

In summary, the measurement of spin diffusion in zero field appears to be potentially useful for structure determination in solid materials where $\mathrm{x}$-ray diffraction methods are inapplicable. Experimentally we have observed the spin diffusion process in diethylterephthalate- $d_{4}$, and a brief theory of spin diffusion in zero field has been described in this chapter. The results show that the rates of spin diffusion depend on the square of the dipolar coupling constant and the relative orientations between the molecular frame and the dipolar principle axis system. Interpretation of the rate is simpler than it is in high field because the rates are independent of the orientation of the molecular frame with respect to the laboratory. Unfortunately, the numerical simulations have not yet been accomplished and are left for future work. 
Table 1.5: Irreducible tensors in the coupled system with two spin $i=1$

\begin{tabular}{|c|}
\hline$T_{0,0}(11)=\frac{1}{\sqrt{3}}\left[T_{1,-1} T_{1,1}-T_{1,0} T_{1,0}+T_{1,1} T_{1,-1}\right]$ \\
\hline$T_{1,0}(11)=\frac{1}{\sqrt{2}}\left[T_{1,1} T_{1,-1}-T_{1,-1} T_{1,1}\right]$ \\
$T_{1, \pm 1}(11)= \pm \frac{1}{\sqrt{2}}\left[T_{1, \pm 1} T_{1,0}-T_{1,0} T_{1, \pm 1}\right]$ \\
\hline$T_{2,0}(11)=\frac{1}{\sqrt{6}}\left[T_{1,-1} T_{1,1}+2 T_{1,0} T_{1,0}+T_{1,1} T_{1,-1}\right]$ \\
$T_{2, \pm 1}(11)=\frac{1}{\sqrt{2}}\left[T_{1,0} T_{1, \pm 1}+T_{1, \pm 1} T_{1,0}\right]$ \\
$T_{2, \pm 2}(11)=T_{1, \pm 1} T_{1, \pm 1}$ \\
\hline \hline$T_{1,0}(12)=\frac{1}{\sqrt{10}}\left[\sqrt{3} T_{1,-1} T_{2,1}-2 T_{1,0} T_{2,0}+\sqrt{3} T_{1,1} T_{2,-1}\right]$ \\
$T_{1, \pm 1}(12)=\frac{1}{\sqrt{10}}\left[\sqrt{6} T_{1, \mp 1} T_{2, \pm 2}-\sqrt{3} T_{1,0} T_{2, \pm 1}+T_{1, \pm 1} T_{2,0}\right]$ \\
\hline$T_{2,0}(12)=\frac{1}{\sqrt{2}}\left[T_{1,1} T_{2,-1}-T_{1,-1} T_{2,1}\right]$ \\
$T_{2, \pm 1}(12)= \pm \frac{1}{\sqrt{6}}\left[\sqrt{3} T_{1, \pm 1} T_{2,0}-\sqrt{2} T_{1, \mp 1} T_{2, \pm 2}-T_{1,0} T_{2, \pm 1}\right]$ \\
$T_{2, \pm 2}(12)= \pm \frac{1}{\sqrt{3}}\left[T_{1, \pm 1} T_{2, \pm 1}-\sqrt{2} T_{1,0} T_{2, \pm 2}\right]$ \\
\hline$T_{3,0}(12)=\frac{1}{\sqrt{5}}\left[T_{1,-1} T_{2,1}+\sqrt{3} T_{1,0} T_{2,0}+T_{1,1} T_{2,-1}\right]$ \\
$T_{3, \pm 1}(12)=\frac{1}{\sqrt{15}}\left[T_{1, \mp 1} T_{2, \pm 2}+\sqrt{8} T_{1,0} T_{2, \pm 1}+\sqrt{6} T_{1, \pm 1} T_{2,0}\right]$ \\
$T_{3, \pm 2}(12)=\frac{1}{\sqrt{3}}\left[T_{1,0} T_{2, \pm 2}+\sqrt{2} T_{1, \pm 1} T_{2, \pm 1}\right]$ \\
$T_{3, \pm 3}(12)=T_{1, \pm 1} T_{2, \pm 2}$ \\
\hline \hline$T_{1,0}(21)=\frac{1}{\sqrt{10}}\left[\sqrt{3} T_{2,-1} T_{1,1}-2 T_{2,0} T_{1,0}+\sqrt{3} T_{2,1} T_{1,-1}\right]$ \\
$T_{1, \pm 1}(21)=\frac{1}{\sqrt{10}}\left[T_{2,0} T_{1, \pm 1}-\sqrt{3} T_{2, \pm 1} T_{1,0}+\sqrt{6} T_{2, \pm 2} T_{1, \pm 1}\right]$ \\
\hline$T_{2,0}(21)=\frac{1}{\sqrt{2}}\left[T_{2,1} T_{1,-1}-T_{2,-1} T_{1,1}\right]$ \\
$T_{2, \pm 1}(21)= \pm \frac{1}{\sqrt{6}}\left[T_{2, \pm 1} T_{1,0}+\sqrt{2} T_{2, \pm 2} T_{1, \mp 1}-\sqrt{3} T_{2,0} T_{1, \pm 1}\right]$ \\
$T_{2, \pm 2}(21)= \pm \frac{1}{\sqrt{3}}\left[\sqrt{2} T_{2, \pm 2} T_{1,0}-T_{2, \pm 1} T_{1, \pm 1}\right]$ \\
\hline$T_{3,0}(21)=\frac{1}{\sqrt{5}}\left[T_{2,-1} T_{1,1}+\sqrt{3} T_{2,0} T_{1,0}+T_{2,1} T_{1,-1}\right]$ \\
$T_{3, \pm 2}(21)=\frac{1}{\sqrt{3}}\left[\sqrt{2} T_{2, \pm 1} T_{1, \pm 1}+T_{2, \pm 2} T_{1,0}\right]$ \\
$\frac{1}{\sqrt{15}}\left[\sqrt{6} T_{2,0} T_{1, \pm 1}+\sqrt{8} T_{2, \pm 1} T_{1,0}+T_{2, \pm 2} T_{1, \mp 1}\right]$ \\
\hline$T_{2, \pm 2} T_{1, \pm 1}$ \\
\hline
\end{tabular}


Table 1.6: Irreducible tensors in the coupled system with two spin $i=1$ (continue)

\begin{tabular}{|c|}
\hline$T_{0,0}(22)=\frac{1}{\sqrt{5}}\left[T_{2,-2} T_{2,2}-T_{2,-1} T_{2,1}+T_{2,0} T_{2,0}-T_{2,1} T_{2,-1}+T_{2,2} T_{2,-2}\right]$ \\
\hline$T_{1,0}(22)=\frac{1}{\sqrt{10}}\left[T_{2,-1} T_{2,1}-2 T_{2,-2} T_{2,2}-T_{2,1} T_{2,-1}+2 T_{2,2} T_{2,-2}\right]$ \\
$T_{1, \pm 1}(22)= \pm \frac{1}{\sqrt{10}}\left[\sqrt{3} T_{2,0} T_{2, \pm 1}-\sqrt{2} T_{2, \mp 1} T_{2, \pm 2}-\sqrt{3} T_{2, \pm 1} T_{2,0}+\sqrt{2} T_{2, \pm 2} T_{2, \mp 1}\right]$ \\
\hline$T_{2,0}(22)=\frac{1}{\sqrt{14}}\left[2 T_{2,-2} T_{2,2}+T_{2,-1} T_{2,1}-2 T_{2,0} T_{2,0}+T_{2,1} T_{2,-1}+2 T_{2,2} T_{2,-2}\right]$ \\
$T_{2, \pm 1}(22)=\frac{1}{\sqrt{14}}\left[\sqrt{6} T_{2, \mp 1} T_{2, \pm 2}-T_{2,0} T_{2, \pm 1}-T_{2, \pm 1} T_{2,0}+\sqrt{6} T_{2, \pm 2} T_{2, \mp 1}\right]$ \\
$T_{2, \pm 2}(22)=\frac{1}{\sqrt{7}}\left[\sqrt{2} T_{2,0} T_{2, \pm 2}-\sqrt{3} T_{2, \pm 1} T_{2, \pm 1}+\sqrt{2} T_{2, \pm 2} T_{2,0}\right]$ \\
\hline$T_{3,0}(22)=\frac{1}{\sqrt{10}}\left[2 T_{2,1} T_{2,-1}+2 T_{2,2} T_{2,-2}-T_{2,-2} T_{2,2}-2 T_{2,-1} T_{2,1}\right]$ \\
$T_{3, \pm 1}(22)= \pm \frac{1}{\sqrt{10}}\left[\sqrt{2} T_{2, \pm 1} T_{2,0}+\sqrt{3} T_{2, \pm 2} T_{2, m p 1}-\sqrt{3} T_{2, \mp 1} T_{2, \pm 2}-\sqrt{2} T_{2,0} T_{2, \pm 1}\right]$ \\
$T_{3, \pm 2}(22)= \pm \frac{1}{\sqrt{2}}\left[T_{2, \pm 2} T_{2,0}-T_{2,0} T_{2, \pm 2}\right]$ \\
$T_{3, \pm 3}(22)= \pm \frac{1}{\sqrt{2}}\left[T_{2, \pm 2} T_{2, \pm 1}-T_{2, \pm 1} T_{2, \pm 2}\right]$ \\
\hline$T_{4,0}(22)=\frac{1}{\sqrt{70}}\left[T_{2,-2} T_{2,2}+4 T_{2,-1} T_{2,1}+6 T_{2,0} T_{2,0}+4 T_{2,1} T_{2,-1}+T_{2,2} T_{2,-2}\right]$ \\
$T_{4, \pm 1}(22)=\frac{1}{\sqrt{14}}\left[T_{2, \mp 1} T_{2, \pm 2}+\sqrt{6} T_{2,0} T_{2, \pm 1}+\sqrt{6} T_{2, \pm 1} T_{2,0}+T_{2, \pm 2} T_{2, \mp 1}\right]$ \\
$T_{4, \pm 2}(22)=\frac{1}{\sqrt{14}}\left[\sqrt{3} T_{2,0} T_{2, \pm 2}+\sqrt{8} T_{2, \pm 1} T_{2, \pm 1}+\sqrt{3} T_{2, \pm 2} T_{2,0}\right]$ \\
$T_{4, \pm 3}(22)=\frac{1}{\sqrt{2}}\left[T_{2, \pm 1} T_{2, \pm 2}+T_{2, \pm 2} T_{2, \pm 1}\right]$ \\
$T_{4, \pm 4}(22)=T_{2, \pm 2} T_{2, \pm 2}$ \\
\hline \hline
\end{tabular}




\section{Chapter 2}

\section{Theory of Zero Field NMR in High Field}

\subsection{Introduction}

It is the dispersion that causes broadened and featureless lineshapes in solid state NMR spectra for polycrystalline or amorphous samples in which there are many chemically and magnetically different nuclei. One of the origins of the dispersion is the anisotropic characters of spin-spin interactions in the samples and is reflected by the orientation dependence of the resonance frequency of each crystal with respect to the external magnetic field. The extraction of structural and dynamical information about the molecules in the sample is then achieved only by applying high resolution solid state NMR techniques, for examples, magic angle spinning (MAS) [56, 57, 58], dynamic angle spinning (DAS) $[78,76]$, double rotation (DOR) $[72,104]$, and multiple pulse (MP) [114]. The principle involved in these methods is the coherently motional averaging under which the orientation dependence of the resonance frequency can be completely or partially removed, and the NMR spectra contain only the isotropic chemical shifts. However, the price paid for applying these methods is that information about anisotropic interactions is thereby lost.

Recently, Pines and his coworkers have developed a new method, time domain zero field NMR and NQR, which can overcome the above limitations while obtaining 
high resolution spectra [8]-[12]. In this method, the sample is pneumatically moved to an intermediate magnetic field $(0.01 T)$ after it is polarized in a high magnetic field $(4.7 \mathrm{~T})$. The intermediate field is then suddenly removed completely when the magnetization of the sample starts to freely evolve under the zero field spin Hamiltonian which is a scalar operator, invariant under a rotation operation to the sample. The resonance frequencies of the magnetization are then orientation independent. After evolution for length of time, $t$, the intermediate field is suddenly turned on and the sample is transferred back to the high field for detection. FID signals can be collected by performing a number of this experiment with a time increment. The main feature applied in time domain zero field NMR is the field cycling technique [7] commonly used in pure nuclear quadrupolar resonance (NQR). Zero field NMR spectra obtained by field cycling technique therefore consist of sharp peaks whose frequencies allow us to measure the principal values of the spin interactions.

The condition involved in time domain zero field NMR and NQR is that the samples must have long relaxation times in order to maintain the polarization, obtained in the high field, of the magnetization in the samples, and the polarization changes arising from the zero field evolution before the detection in the high field. Furthermore, in zero field, it is difficult to selectively excite a particular nuclear species because the Larmor frequencies of all nuclear species are equal to zero in the zero field. The main reason of these two problems is because of the application of field cycling technique. The question therefore arises whether it is possible to directly obtain zero field NMR spectra in the high field without ever taking the sample out of the magnet. Indeed, in series of studies, Tycko has recently shown that this is possible $[49,50,51]$. The possibility originates from the fact that in a coupled space, made up of the direct product of spatial and spin coordinates, the high field spin Hamiltonian can be represented by a linear combination of irreducible tensors from rank zero to four. Among them, the zero rank tensor which carries 
both of isotropic and anisotropic information about the spin interaction transforms like a scalar operator. In other words, the zero rank tensor is exactly equivalent to the zero field spin Hamiltonian, and may yield high resolution spectra. Thus the strategy to accomplish the zero field NMR in high field experiment is to average out the high rank tensors and leaves only the scalar part of the total Hamiltonian. In this chapter, I first review the theory of zero field NMR in high field given by Tycko [51], based on coherent average Hamiltonian theory[115]. The parameters used in Tycko's pulse sequence of zero field NMR in high field is then analytically solved. These solutions are critical to achieve high quality of the zero field NMR spectra in high field. In section 2.4, I present a new trajectory of zero field NMR in high field. After that I turn into describing theoretically optimized solutions: dynamic angle hopping (DAH), dynamic angle spinning (DAS), and double rotation (DOR) in the coupled space for zero field NMR in high field.

\subsection{Reconstructing Scalar Hamiltonian in High Field}

A zero field dipolar or quadrupolar spin Hamiltonian can be represented by an inner product of two second rank tensors: one reflecting the spatial behavior and the other reflecting the spin behavior of the dipolar or quadrupolar interaction,[5]. In terms of the irreducible tensors, the spin Hamiltonian can be written as [19]

$$
\mathcal{H}=\sum_{m=-2}^{2}(-1)^{m} A_{2,-m} T_{2, m}
$$

where $T_{2, m}$ and $A_{2, m}$ are components of a second rank spin and spatial tensor respectively. Although Eq.(2.1) involves orientation dependent terms, the whole expression of the Hamiltonian is invariant under a rotation operation. After application of a strong external magnetic field along a particular direction (the $z$-axis) in the laboratory frame, all spins prefer to align up along the $z$-axis and the rotational symmetry of the spin Hamiltonian is reduced from $S O(3)$ to $C_{\infty}$ (along the $z$-axis). In other 
words, because the interaction between the spins and the external magnetic field, the Zeeman interaction, is usually so large that the internal spin Hamiltonian can be efficiently truncated. To explicitly represent the truncated Hamiltonian, we first transform the spin Hamiltonian from the laboratory frame to the rotating frame (that is, the interaction picture) by the unitary operator $e^{-\imath \mathcal{H}_{z} t}$, where $\mathcal{H}_{z}$ is the Zeeman Hamiltonian. The spin Hamiltonian in the rotating frame is then time dependent, and under the first order perturbation approximation, the time dependent parts are ignored and leave only the time independent term of the total spin Hamiltonian. In other words, the spin Hamiltonian becomes

$$
\mathcal{H}=A_{2,0} T_{2,0}
$$

In order to reconstruct a scalar Hamiltonian from Eq.(2.2), it is possible to represent the spin Hamiltonian in terms of a single set of irreducible tensors, $\left\{\mathcal{F}_{l, m}\right\}$, which are the product tensors of the $A_{2, m}$ and $T_{2, m}$. This is equivalent to transform the spin Hamiltonian from two separately spaces (spin, and spatial spaces) into the coupled spatial-spin space, the product space of the spatial and spin spaces, and the $\left\{\mathcal{F}_{l, m}\right\}$ are tensors in the coupled spatial-spin space. Using the multiplication properties of two irreducible tensors[20,116,117, 118], the product of the $A_{2, m}$ and $T_{2, m}$ is given by

$$
A_{l_{1}, m_{1}} T_{l_{2}, m_{2}}=\sum_{l=\left|l_{1}-l_{2}\right|}^{l_{1}+l_{2}} C\left(l_{1}, l_{2}, m_{1}, m_{2}\right) \mathcal{F}_{l, m_{1}+m_{2}}
$$

where $C\left(l_{1}, l_{2}, m_{1}, m_{2}\right)$ are the Clebsch-Gordan coefficients. Inserting Eq.(2.3) into Eq.(2.2) yields

$$
\mathcal{H}=\sum_{l=0,2,4} C(2,2, l, 0,0) \mathcal{F}_{l, 0}
$$

where no odd rank tensors appear in the Hamiltonian due to the symmetry properties of the spin interaction. The zero order tensor $\mathcal{F}_{0,0}$ corresponds to the orientally invariant component of the internal spin Hamiltonian, i.e., the scalar component usually involved in zero field NMR. The Clebsch-Gordan coefficient of $\mathcal{F}_{0,0}$ is $\frac{1}{\sqrt{5}}$, 
but the $\mathcal{F}_{l, m}$ tensors are not normalized. After normalization of the $\mathcal{F}_{l, m}$ tensors, the coefficient of the scalar component should be one firth. This implies that the strength of the spin interaction will be scaled down by a factor of five, and results in that the resonance frequencies will be reduced five times in comparison with the zero field NMR spectra obtained by time domain zero field NMR approach. Although the remaining terms in Eq.(2.4) are orientation dependent, they can be removed by using the motional average techniques in high resolution soljd state NMR [19] with some necessary modifications, for example, the sample spinning synchronized by the applied pulse sequence. In the following paragraphs, I discuss how the zero field NMR in high field can be achieved.

A rotation operator in the coupled spatial-spin space can be written as

$$
\mathcal{R}(\Omega)=R\left(\Omega_{R}\right) P\left(\Omega_{P}\right)
$$

where $R\left(\Omega_{R}\right)$ represents a mechanical sample rotation and $P\left(\Omega_{P}\right)$ is a spin rotation arising from rf pulses. All rotation operators in Eq.(2.5) are defined by Euler angles: $\alpha$ is for the spin angle, $\beta$ for the nutation angle, and $\gamma$ for the precession angle. Application of the rotation operator $\mathcal{R}(\Omega)$ to the spin Hamiltonian (2.4) yields

$$
\mathcal{H}=\sum_{l=0,2,4} \sum_{m=-l}^{l} C(2,2, l, 0,0) \mathcal{D}_{m, 0}^{(l)}(\Omega) \mathcal{F}_{l, m}
$$

where $\mathcal{D}_{m, 0}^{(l)}(\Omega)$ are the components of Wigner rotation matrices. Since in Eq.(2.6) one of the two indices of the Wigner rotation matrices is zero, it is possible to disregard one, the $\gamma$, of the three Euler angles involved in the averaging process. However, the appearance of Wigner rotation matrices in Eq.(2.6) still provides two freedoms, the rotation angle, $\alpha$, and the rotation axis angle, $\beta$, in the manipulation of the spin Hamiltonian. Furthermore, we can also use a set of rotations in the coupled spatial-spin space to average the spin Hamiltonain, so that,

$$
\overline{\mathcal{H}}=\frac{1}{N} \sum_{k=1}^{N} \sum_{l=0,2,4} \sum_{m=-l}^{l} C(, 2,2, l, 0,0) \mathcal{D}_{m, 0}^{(l)}\left(\Omega_{k}\right) \mathcal{F}_{l, m}=\sigma \mathcal{F}_{0,0}
$$


where $\sigma$ is a scaling factor (its maximum value is $\frac{1}{5}$ if $\mathcal{F}_{0,0}$ is normalized), and $\Omega_{k}$ are Euler angles of the $k$-th rotation. Any choice of $N$ and $\Omega_{k}$ which satisfies Eq. (2.7) will be a trajectory for zero field NMR in high field. However, in order to find the trajectories, it is necessary to have a general procedure described as follows.

In the first step, we assume that a number of rotations, $N_{\alpha}$, is applied along a fixed axis inclined at the angle, $\beta_{k}$, with respect to the $z$-axis in the coupled spatialspin space. The average over the $N_{\alpha}$ rotations results in the truncation of the spin Hamiltonian (2.6), which is

$$
\overline{\mathcal{H}}\left(\beta_{k}\right)=\frac{1}{N_{\alpha}} \sum_{l=0,2,4} C(, 2,2, l, 0,0) d_{0,0}^{(l)}\left(\beta_{k}\right) \mathcal{F}_{l, 0}
$$

where $d_{0,0}^{(l)}\left(\beta_{k}\right)$ are the reduced Wigner rotation matrices.

If the applied rotations are discrete, that is, a rotation with finite sizes of the angles is instantaneously applied at particular time, resulting in Eq.(2.8) has to be subjected to the condition of

$$
\sum_{k^{\prime}=1}^{l+1} e^{-\imath m \alpha_{k^{\prime}}}=\left\{\begin{array}{ll}
l+1 & \text { if } m=0 \\
0 & \text { if } m \neq 0
\end{array},\right.
$$

where the rotation angles are

$$
\alpha_{k^{\prime}}=\frac{2 \pi k^{\prime}}{l+1}
$$

and $N_{\alpha}=l+1$, and $l$ is the highest rank of the irreducible tensors in the spin Hamiltonian. In our case, $l=4$ and $N_{\alpha}=5$. This means that the rotations involved in the truncation of Eq.(2.6) should possess at least a five-fold symmetry.

If the applied rotations are continuous, that is, it arises from the sample spinning with a rate $\omega_{r}$, the average over a whole rotation cycle is

$$
\int_{0}^{\frac{2 \pi}{\omega_{r}}} e^{-\imath m \omega_{r} t} d t=\left\{\begin{array}{ll}
\frac{2 \pi}{\omega_{r}} & \text { if } m=0 \\
0 & \text { if } m \neq 0
\end{array},\right.
$$

where $\alpha=\omega_{r} t$, and $N_{\alpha}=2 \pi / \omega_{r}$, the normalization factor. 
In the next step, we choose a suitable set of rotation axes, $\left\{\beta_{k}\right\}$. The average over the rotation axes, $\left\{\beta_{k}\right\}$ can eventually result in the scalar Hamiltonian given in Eq.(2.7). If the rotations are discrete, the set of the rotation axis angles, $\left\{\beta_{k}\right\}$, has to satisfy

$$
\frac{1}{N_{\alpha} N_{\beta}} \sum_{k} C(2,2, l, 0,0) d_{0,0}^{(l)}\left(\beta_{k}\right)=\left\{\begin{array}{ll}
\sigma & \text { if } l=0 \\
0 & \text { if } l \neq 0
\end{array},\right.
$$

where $N_{\beta}$ is the number of rotation axes. Alternatively, if the time dependence of the rotation axes can be made continuous by rotating the sample about two or more axes simultaneously, the condition to obtain a scalar Hamiltonian becomes

$$
\frac{1}{N_{\alpha}} C(2,2, l, 0,0) \prod_{k} d_{0,0}^{(l)}\left(\beta_{k}\right)=\left\{\begin{array}{ll}
\sigma & \text { if } l=0 \\
0 & \text { if } l \neq 0
\end{array},\right.
$$

where $N_{\beta}$ is one. Eqs (2.9)-(2.13) present four general conditions for establishing trajectories capable of affording zero field NMR spectra in high field. In the next sections we discuss five different trajectories recently proposed by Tycko as well as by our group.

\subsection{Exact Solutions of Tycko's Pulse Sequence}

The first trajectory for obtaining zero field NMR spectra in high field was proposed by Tycko in 1988 on the basis of a numerical computer search [49]. In his original experiment $[50,51]$, the sample rotates around an axis inclined at the angle, $\beta_{r}=75^{\circ}$, with respect to the external field and the five-fold symmetry pulse sequence shown in Figure (2.1) is applied to the spin Hamiltonian. The five-fold symmetry pulse sequence consists of five blocks, and is synchronized with the sample spinning. Each block has a phase increment, $72^{\circ}$, and contains two pairs of delta rf pulses. Each pair with two pulses forms a discrete spin rotation to the spin operators of the spin Hamiltonian. The axes of all spin rotations are in the $x y$-plane of the rotating frame and the phases of the two spin rotation axes in the $k$-th block are $\varphi_{2 k}=2 k \varphi+\phi_{1}$ and $\varphi_{2 k+1}=2 k \varphi+\phi_{2}$ where $\varphi=\omega_{r} \tau=36^{\circ}$ and $2 \tau$ is the length of time involved 
in each block, and $\phi_{1}=0^{\circ}, \phi_{2}=-22^{\circ}$, the initial phases of the two rotation axes of the first block. The nutation angles of all spin rotations are $\beta_{1}=\beta_{2}=\beta_{p}=46^{\circ}$. This pulse sequence afforded a high resolution zero field NMR spectrum of a $95 \%$ deuterated sulid benzene sample in high field. This spectrum consists of three peaks whose splitting is equal to the dipole-dipole coupling constant of the two protons in benzene, multiplied by a scaling factor of 0.089 . In the present section we rederive Tycko's pulse sequence within the general description of the method.

We assume that the sample rotates around a fixed axis oriented at an arbitrary angle with respect to the external magnetic field, and consider what kind of pulse sequence is needed to make the zeroth order average Hamiltonian a scalar. According to group theory $[68,116]$, five orientations equally distributed on a $2 \pi$ period can average out up to fourth rank tensors with the exception of the zero rank tensor which is a scalar. The application of the five-fold symmetry to our case is accomplished by applying five rotational operations to the spin Hamiltonian in the coupled spatialspin space. Each rotation has an increment, $72^{\circ}$, of the spin angle, $\alpha$, relative to the previous one. The average over the five-fold symmetry rotation operations removes the $\alpha$ dependence in the spin Hamiltonian. Practically, this can be implemented in a way that one rotational cycle is divided into five equal arcs separated by $72^{\circ}$ increment. Within each arc, the pulse sequence shown in Figure $(2.1 \mathrm{c})$ is applied to spin operators of the spin Hamiltonian. This sequence consists of four pulses and involves two discrete spin rotation operators with fixed rotation axis angles (the nutation angles, $\beta$ ). Physically, the trajectory of the variation of the spin Hamiltonian as the sample continuously rotates depend on not only the spin Hamiltonian and spinning speeds but also the initial position of the sample in the laboratory frame. According to average Hamiltonian theory [115], we do not sare the exact trajectory of the variations of the spatial tensors during one spinning period. What is important for us is the initial and final values of the spatial tensors in one spinning period, 
A

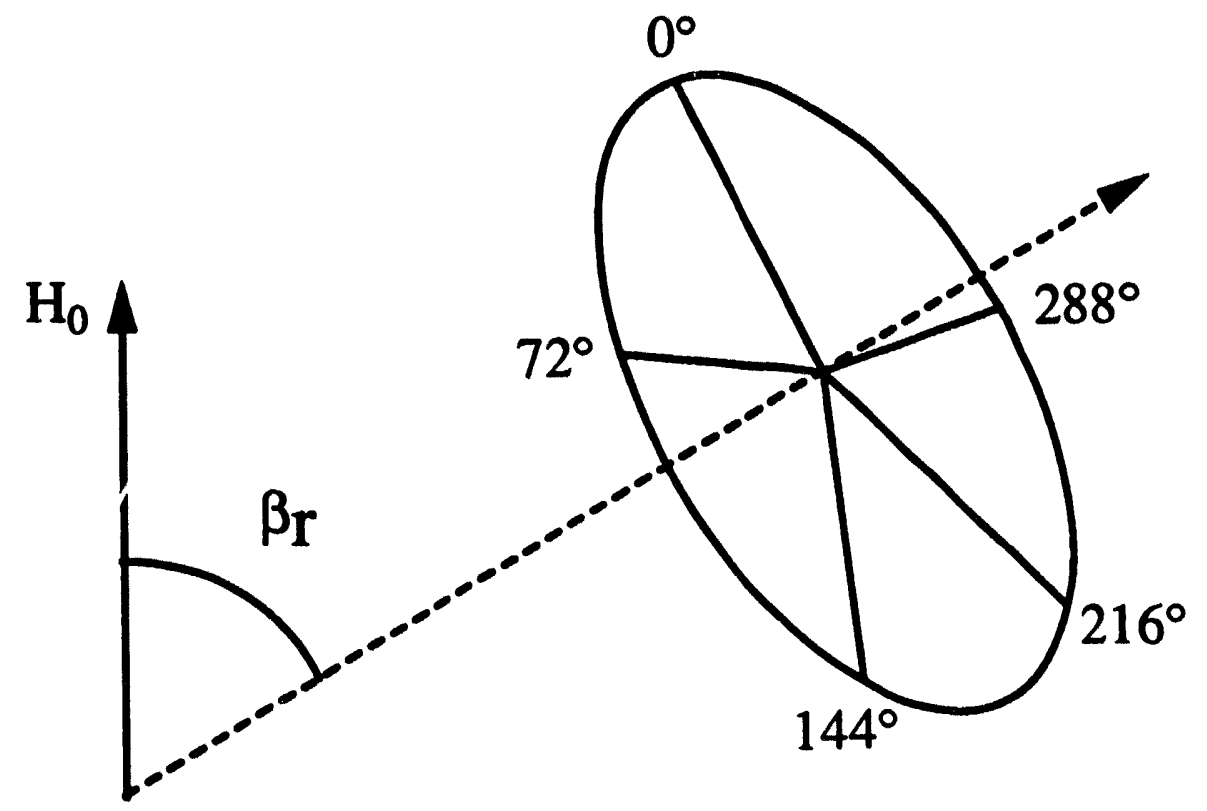

B

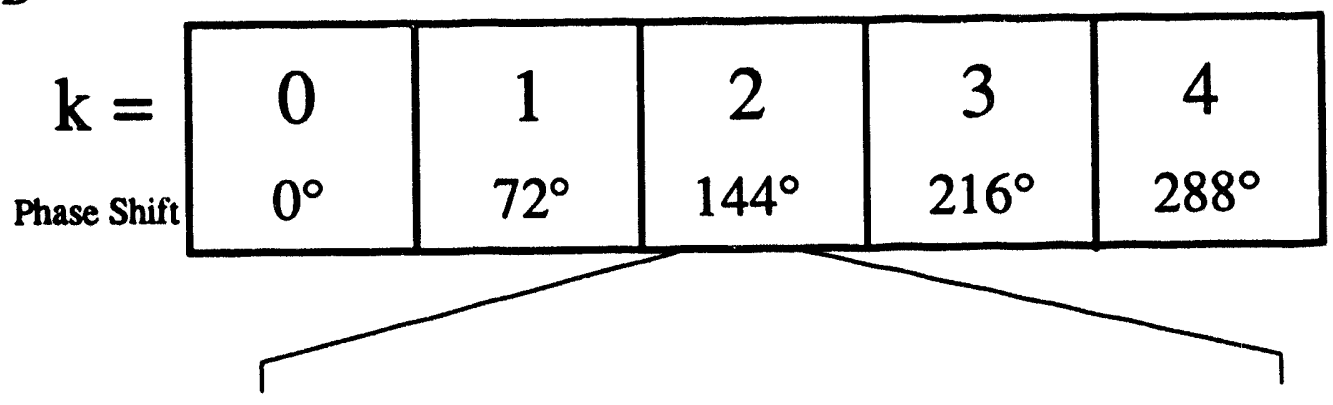

$\mathbf{C}$

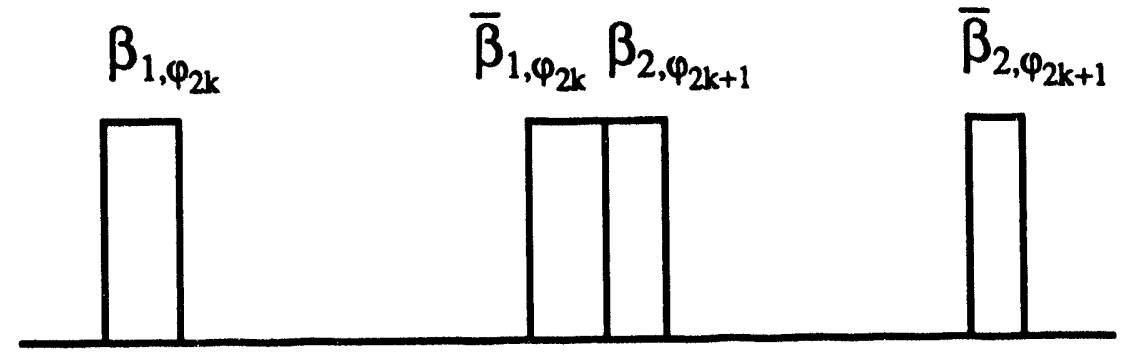

Figure 2.1: Generalized Tycko's trajectory. (A) The rotor cycle, the sample rotates at an angle $\beta_{r}$ with respect to the external field and an angular velocity $\omega_{r}=\frac{2 \pi}{10 \tau}$. (B) The synchronized pulse sequence, each block is phase shifted by $72^{\circ}$ from the previous one, and $(C)$ details of one pulse sequence block, where $\beta_{1}, \beta_{2}$ are the flipping angles with phase $\varphi_{2 k}=2 k \varphi+\phi_{1}, \varphi_{2 k+1}=2 k \varphi+\phi_{2}$, and $\varphi=\omega_{r} \tau=36^{\circ}$. 
and these two values are obviously dependent on both the spinning speed and the initial position of the sample. As was described in the last section, in order to form a scalar Hamiltonian, the spin tensors should have the same values as those of the spatial tensors at the beginning and at the end of a period. This is the reason why two discrete rotation operators for the spin operators in the Hamiltonian are needed: one is used to control the initial phase and the other determines the final phase of the spin tensors in each rotation section.

The propagator of the pulse sequence applied during $k$-th rotation arc (block) of a period is

$$
\mathcal{L}_{k}(\tau)=e^{-\imath \beta_{2} I_{\varphi_{2 k+1}}} e^{-\imath \overline{\mathcal{H}}_{2 k+1}(\tau)} e^{\imath \beta_{2} I_{\varphi 2 k+1}} e^{-\imath \beta_{1} I_{\varphi_{2 k}}} e^{-\imath \overline{\mathcal{H}}_{2 k}(\tau)} e^{\imath \beta_{1} I_{\varphi 2 k}}
$$

where

$$
\begin{aligned}
\varphi_{2 k+1} & =2 k \varphi+\phi_{1} \\
\varphi_{2 k} & =2 k \varphi+\phi_{2} \\
\varphi & =\omega_{r} \tau=36^{\circ},
\end{aligned}
$$

and $\phi_{1}$ and $\phi_{2}$ are constant phases for each pulse. The zero order average Hamiltonian, $\overline{\mathcal{H}_{k}}(\tau)$ over the sample spinning in the time interval $\tau$ is

$$
\overline{\mathcal{H}}_{k}(\tau)=\sum_{m} B_{m}(\tau) e^{-\imath k m \omega_{r} \tau} A_{2, m} T_{2,0}
$$

where

$$
B_{m}(\tau)= \begin{cases}\frac{2}{m \omega_{r}}\left(e^{-\imath m \omega_{r} \tau}-1\right) d_{m, 0}^{(2)}\left(\beta_{r}\right) & \text { for } m \neq 0 \\ d_{0,0}^{(2)}\left(\beta_{r}\right) \tau & \text { for } m=0\end{cases}
$$

and $\beta_{r}$ is the angle of the rotation axis with respect to the external field.

According to average Hamiltonian theory, by the end of a cycle the total propagator under zero order approximation is

$$
\mathcal{L}\left(\tau_{c}\right)=\prod_{k=0}^{4} \mathcal{L}_{k}(\tau)=e^{-i \overline{\mathcal{H}} \tau_{c}}
$$


Since

$$
\sum_{k=0}^{4} e^{-i k\left(m+m^{\prime}\right) \varphi}=\left\{\begin{array}{ll}
0 & \text { if } m+m^{\prime} \neq 0 \\
5 & \text { if } m+m^{\prime}=0
\end{array},\right.
$$

Eqs. (2.14) - (2.17) allow us to write the total zero order average Hamiltonian, $\overline{\mathcal{H}}$, as

$$
\begin{aligned}
\overline{\mathcal{H}} & =\frac{1}{\tau_{c}} \sum_{m, m^{\prime}} B_{m}\left[\sum_{k=0}^{4} e^{-i k\left(m+m^{\prime}\right) \varphi}\right]\left[e^{-\imath m^{\prime} \phi_{1}} d_{m^{\prime}, 0}^{(2)}\left(\beta_{1}\right)+e^{-\mathrm{i}\left(m \omega_{r} \tau+m^{\prime} \phi_{2}\right)} d_{m^{\prime}, 0}^{(2)}\left(\beta_{2}\right)\right] A_{2, m} T_{2, m^{\prime}} \\
& =\frac{5}{\tau_{c}} \sum_{m} B_{-m}\left[e^{-\mathrm{sm} \phi_{1}} d_{m, 0}^{(2)}\left(\beta_{1}\right)+e^{-\mathrm{s} m\left(\phi_{2}-\omega_{r} \tau\right)} d_{m, 0}^{(2)}\left(\beta_{2}\right)\right] A_{2,-m} T_{2, m}
\end{aligned}
$$

From Eq.(2.20), the zero order average Hamiltonian becomes a scalar operator only if

$$
\frac{5}{\tau_{c}} B_{-m}\left[e^{-\imath m \phi_{1}} d_{m, 0}^{(2)}\left(\beta_{1}\right)+e^{-\imath m\left(\phi_{2}-\omega_{r} \tau\right)} d_{m, 0}^{(2)}\left(\beta_{2}\right)\right]=(-1)^{m} \sigma \quad m=-2, \cdots, 2
$$

where $\sigma$ is a scaling factor. In Eq. (2.21), there are six unknowns $\left(\beta_{r}, \beta_{1}, \beta_{2}, \phi_{1}, \phi_{2}\right.$, $\sigma$ ) and five complex simultaneous equations for different $m$. Due to the symmetry of Wigner rotation matrices, only three equations are independent, which can be written as five real simultaneous equations (for $m=0$, the equation is already real). Only five unknowns can be determined by these equations, and therefore one out of the six unknowns varies as a free variable. By redefining the phase variables as

$$
\begin{aligned}
\phi_{1}^{\prime} & =\phi_{1}-\frac{1}{2} \varphi \\
\phi_{2}^{\prime} & =\phi_{2}-\frac{3}{2} \varphi
\end{aligned}
$$

where $\varphi=\omega_{r} \tau=36^{\circ}$, it is possible to express the five real equations as

$$
\left\{\begin{array}{l}
\frac{1}{2} d_{0,0}^{(2)}\left(\beta_{r}\right)\left[d_{0,0}^{(2)}\left(\beta_{1}\right)+d_{0,0}^{(2)}\left(\beta_{2}\right)\right]=\sigma \\
-\frac{5}{\pi} \sin \left(\frac{1}{2} \phi\right) d_{-1,0}^{(2)}\left(\beta_{r}\right)\left[d_{1,0}^{(2)}\left(\beta_{1}\right) \cos \left(\phi_{1}^{\prime}\right)+d_{1,0}^{(2)}\left(\beta_{2}\right) \cos \left(\phi_{2}^{\prime}\right)\right]=\sigma \\
\frac{5}{2 \pi} \sin (\phi) d_{-2,0}^{(2)}\left(\beta_{r}\right)\left[d_{2,0}^{(2)}\left(\beta_{1}\right) \cos \left(2 \phi_{1}^{\prime}\right)+d_{2,0}^{(2)}\left(\beta_{2}\right) \cos \left(2 \phi_{2}^{\prime}\right)\right]=\sigma \\
d_{1,0}^{(2)}\left(\beta_{1}\right) \sin \left(\phi_{1}^{\prime}\right)+d_{1,0}^{(2)}\left(\beta_{2}\right) \sin \left(\phi_{2}^{\prime}\right)=0 \\
d_{2,0}^{(2)}\left(\beta_{1}\right) \sin \left(2 \phi_{1}^{\prime}\right)+d_{2,0}^{(2)}\left(\beta_{2}\right) \sin \left(2 \phi_{2}^{\prime}\right)=0
\end{array}\right.
$$


Eq.(2.23) can be easily solved in the case of $\beta_{1}=\beta_{2}=\beta_{p}$, as the solution is determined by the following quadratic equation

$$
x^{2}+B x+C=0
$$

where

$$
\begin{aligned}
x & =\tan ^{2}\left(\beta_{r}\right) \\
B & =\frac{1}{2}\left[\frac{15}{2 \pi} \sin (\phi) \cos \left(2 \phi_{1}^{\prime}\right)-1\right] C-2 \\
C & =\frac{16 \cos ^{2}\left(\phi_{1}^{\prime}\right)}{\cos ^{2}\left(\frac{1}{2} \phi\right) \cos ^{2}\left(2 \phi_{1}^{\prime}\right)}
\end{aligned}
$$

and $\phi_{1}^{\prime}$ is a free parameter which can be any value between 0 to $2 \pi$. After obtain $\beta_{r}$ from Eqs. (2.24) and (2.25), the other two variables, $\beta_{p}$ and $\phi_{2}^{\prime}$, can be calculated as

$$
\left\{\begin{array}{l}
\tan \left(\beta_{r}\right) \tan \left(\beta_{p}\right)= \pm \sqrt{C} \\
\phi_{2}^{\prime}=-\phi_{1}^{\prime}
\end{array} .\right.
$$

Moreover, from Eq.(2.25), it results that the coefficients $B$ and $C$ have a period of $\pi$ with respect to the $\phi_{1}^{\prime}$, that is, the values of the coefficients B and C at $\phi_{1}^{\prime}$ are the same as at $\phi_{1}^{\prime}+\pi$.

We have numerically solved the five simultaneous equations (2.20) for the case of $\beta_{1} \neq \beta_{2}$ by use of computer. However, the results show that the largest scaling factor is always found when $\beta_{1}=\beta_{2}$. We are, therefore, not interested in the case of $\beta_{1} \neq \beta_{2}$.

Figure (2.2) shows $\beta_{r}, \beta_{p}$, and $\sigma$ as functions of $\phi_{1}^{\prime}$. From the figure it can be clearly seen that solutions of $\beta_{r}, \beta_{p}$, and $\sigma$ exist only in the region of $\phi_{1}^{\prime} \in$ $\left(32.69^{\circ}, 147.31^{\circ}\right)$. In principle, solutions of $\beta_{r}$ and $\beta_{p}$ can be replaced with each other. However, for practical convenience, we define the angle of the sample rotation axis, $\beta_{r}$, to be in the range of $0^{\circ}$ to $90^{\circ}$. The pulse angle $\beta_{p}$ has two sets solutions varying from $-71^{\circ}$ to $71^{\circ}$ and each set exhibits an inverse symmetry about $\beta_{p}=0^{\circ}$ and $\phi^{\prime}=90^{\circ}$. The solutions of $\beta_{r}$ and $\sigma$ are symmetric around $\phi^{\prime}=90^{\circ}$. 

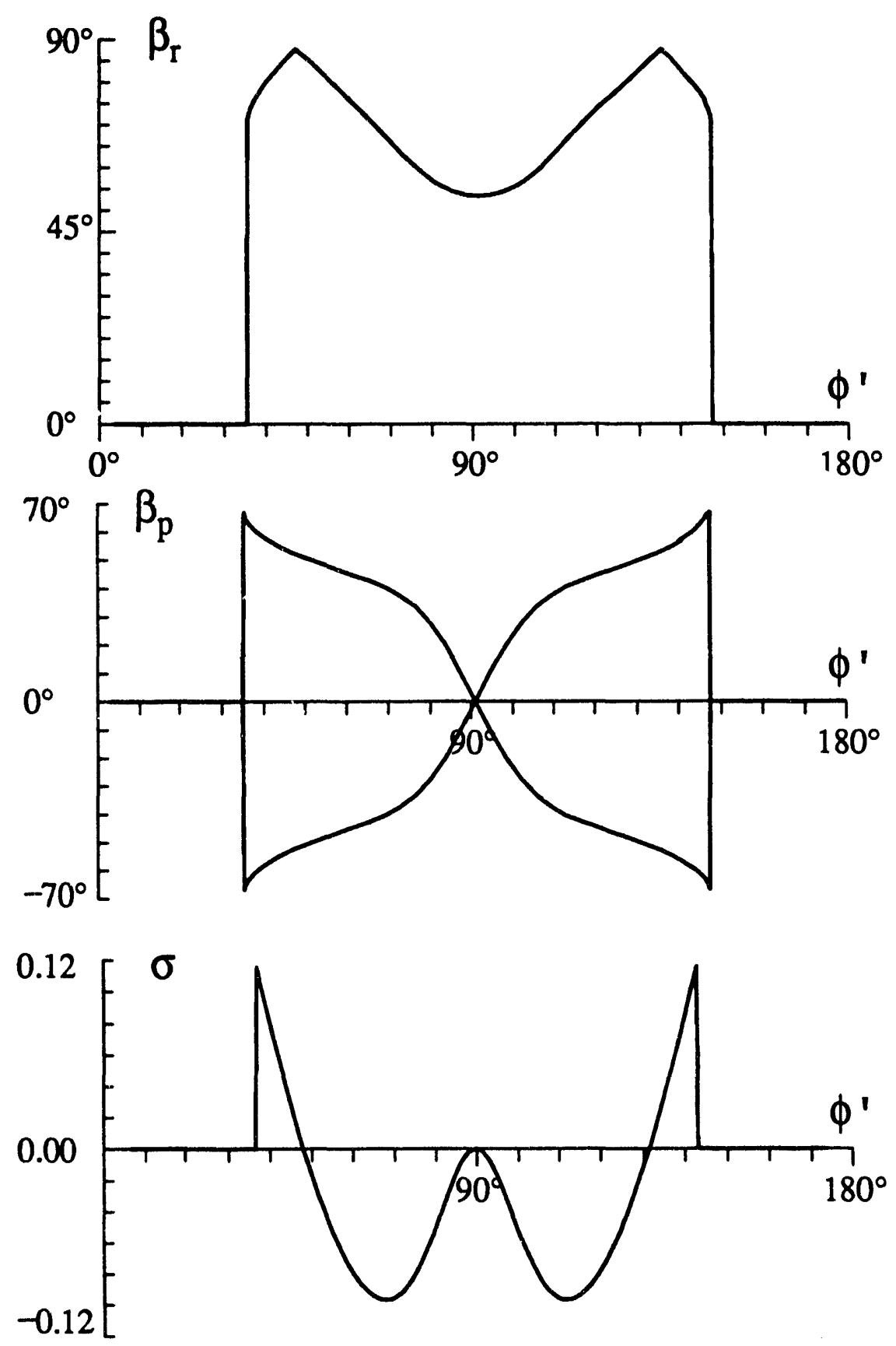

Figure 2.2: Exact solutions of $\beta_{r}, \beta_{p}\left(\beta_{1}=\beta_{2}=\beta_{p}\right)$, and $\sigma$ as functions of free variable $\phi_{1}^{\prime}$ in period $\left[0^{\circ}, 180^{\circ}\right]$. The zero order average Hamiltonian is a scalar operator scaled down by a factor of $\sigma$ from the untruncated internal spin Hamiltonian for each set of $\phi_{1}^{\prime}, \beta_{r}$, and $\beta_{p}$. 
The largest absolute value of the scaling factor $\sigma$ is located at $\phi_{1}^{\prime}=-\phi_{2}^{\prime}=$ $32.689^{\circ}$, or $\phi_{1}=50.689^{\circ}$ and $\phi_{2}=21.311^{\circ}$, with $\beta_{r}=\beta_{p}=71.064^{\circ}$, and at this point $\sigma=0.117$. A more stable solution is found at $\beta_{r}=69.505^{\circ}, \beta_{p}=42.864^{\circ}$, and $\phi_{1}=84.375^{\circ}, \phi_{2}=-12.375^{\circ}$ where $\sigma=-0.0966$. Another stable solution corresponds to the magic angle at which $\sigma=0$.

In Figure (2.3a), the static powder patterns for the asymmetry parameter, $\eta=0$ and $\eta=0.5$ are presented. Figure (2.3b) shows the corresponding high resolution spectra containing the same information. Experimentally, zero field NMR spectra in high field can only be achieved when the spinning speed $\omega_{r}$ is large compared with the internal spin interactions (dipolar or quadrupolar coupling). This will be the major obstacle for applying this method to extract the principal values of the quadrupolar interactions. However, the experiments performed by Tycko et.al[50, 51]. have shown the potential applications of the method for studying coupled lonepair proton systems in which the dipolar couplings have been scaled down by the internal random motions.

\subsection{A New Sequence for Zero Field NMR in High Field}

In Tycko's trajectory, a mechanical sample rotation is synchronized with discrete pulses to obtain an effective scalar Hamiltonian. In this section, I describe an alternative trajectory also based on average Hamiltonian theory, in which both of the spatial and spin parts of the spin Hamiltonian are modulated by continuous rotations, but one of the rotations is allowed to suddenly change the orientation of the rotation axis, and the phase of the spinning. In practice this feature should be used on the spin rotation as it is very difficult to mechanically change the spatial sample

rotation axis. Another feature used in the new trajectory is that the total average Hamiltonian is obtained over two rotation cycles. This feature may affect the eff- 


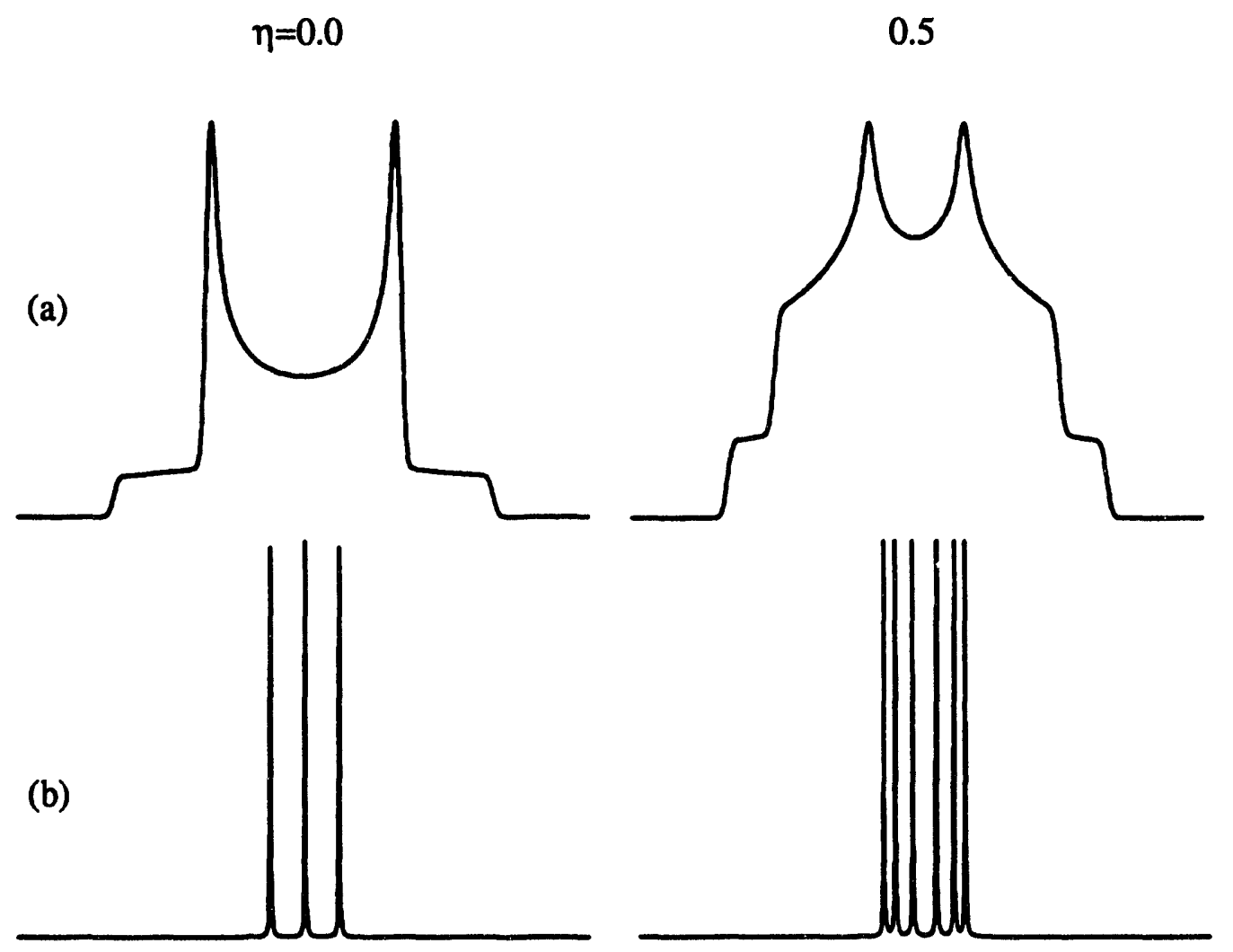

Figure 2.3: Computer simulated (a) powder patterns for $\eta=0$ and $\eta=0.5$, and (b) "zero field" spectra in rotating frame by applying synchronized rotation and pulse sequence $\left(\phi_{1}=84.375, \beta_{r}=69.505, \beta_{p}=42.864\right.$, and $\left.\sigma=-0.0966\right)$ for $\eta=0$ and $\eta=0.5$. 
ciency of the sample spinning in terms of a single spin interaction in the spin system. However, if the chemical shift anisotropic (CSA) interaction is considered, Tycko's trajectory is also needed two cycles in order to apply a $\pi$ pulse between the two cycles to eliminate the CSA interaction.

We start with the rotating frame spin Hamiltonian given by Eq.(2.2). Application of spatial and spin rotations to this Hamiltonian yields

$$
\begin{aligned}
\mathcal{H}(t) & =\sum_{m_{1}, m_{2}} \mathcal{D}_{m_{2}, 0}^{(2)}\left(\Omega_{r}\right) \mathcal{D}_{m_{2}, 0}^{(2)}\left(\Omega_{p}\right) A_{2, m_{1}} T_{2, m_{2} 2} \\
& =\sum_{m_{1}, m_{2}} e^{-\mathrm{z}\left(m_{1} \omega_{r}+m_{2} \omega_{p}\right) t} e^{-\imath\left(m_{1} \varphi_{r}+m_{2} \varphi_{p}\right)} d_{m_{1}, 0}^{(2)}\left(\beta_{r}\right) d_{m_{2}, 0}^{(2)}\left(\beta_{p}\right) A_{2, m_{1}} T_{2, m_{2}},(2,
\end{aligned}
$$

where $\omega_{r}$ and $\omega_{p}$ are the spinning speeds of the spatial and spin rotations respectively, $\varphi_{r}$ and $\varphi_{p}$ are the initial phase of the rotations, and $\beta_{r}$ and $\beta_{p}$ are the angles of the rotation axes with respect to the external field. In order to obtain a scalar Hamiltonian, the indices of the spatial and spin tensors have to satisfy the relationship given in Eq.(2.1): $m_{2}=-m_{1}=m$. The spinning speeds of the two rotations therefore have to be equal, that is, $\omega_{r}=\omega_{p}=\omega_{b}$, and the resulting zero order average Hamiltonian is given by

$$
\overline{\mathcal{H}}=\sum_{m} e^{-\imath m\left(\varphi_{p}-\varphi_{r}\right)} d_{-m, 0}^{(2)}\left(\beta_{r}\right) d_{m, 0}^{(2)}\left(\beta_{p}\right) A_{2,-m} T_{2, m} .
$$

It could be proved that for single pair of rotations around fixed axes it is impossible to obtain the zero field scalar Hamiltonian given by Eq.(2.1). The next step is to consider whether two pairs of rotations can extract the scalar operator from the total spin Hamiltonian in high field. As in the preceding section, we choose to keep the angle of the spatial rotation axis with respect to the external field constant and allow to change the phase and axis of the spin rotation. After application of the average Hamiltonian theory over two rotation cycles, the problem is reduced to finding the solution of the following equation:

$$
d_{-m, 0}^{(2)}\left(\beta_{r}\right)\left[e^{-\imath m \varphi_{p_{1}}} d_{m, 0}^{(2)}\left(\beta_{p_{1}}\right)+e^{-\imath m \varphi_{p_{2}}} d_{m, 0}^{(2)}\left(\beta_{p_{2}}\right)\right]=(-1)^{m} \sigma
$$


where we already set $\varphi_{r}=0$.

Looking for the simplest solutions of Eq.(2.29), we set $\varphi_{p_{1}}=\varphi_{p_{2}}=0$. Eq.(2.29) is then simplified to the following set of three simultaneous equations:

$$
\left\{\begin{array}{l}
d_{0,0}^{(2)}\left(\beta_{r}\right)\left[d_{0,0}^{(2)}\left(\beta_{p_{1}}\right)+d_{0,0}^{(2)}\left(\beta_{p_{2}}\right)\right]=\sigma \\
d_{-1,0}^{(2)}\left(\beta_{r}\right)\left[d_{1,0}^{(2)}\left(\beta_{p_{1}}\right)+d_{1,0}^{(2)}\left(\beta_{p_{2}}\right)\right]=-\sigma \\
d_{-2,0}^{(2)}\left(\beta_{r}\right)\left[d_{2,0}^{(2)}\left(\beta_{p_{1}}\right)+d_{2,0}^{(2)}\left(\beta_{p_{2}}\right)\right]=\sigma
\end{array}\right.
$$

Compared with Eq.(2.23), Eq.(2.30) not only looks simpler but also there are only four unknown variables, and since one of them is independent, it can be chosen as a free variable.

The dependence of the solutions of Eq. (2.30), $\left(\beta_{p_{2}}, \beta_{p_{1}}, \sigma\right)$ with respect to the free variable $\beta_{p_{1}}$ are shown in Figure (2.4). As can be seen from the figure, at magic angle spinning the scaling factor is zero. The maximum scaling factor that can be obtained from this trajectory is 0.17 , but consider the average in the two rotation cycles the actual maximum scaling factor is only about 0.085 , smaller than 0.117 in Tycko's trajectory. Nevertheless, this trajectory possess the advantage that the pulse sequence is very simple and that the point with maximum scaling factor is stable. The most interesting solution of this trajectory is at $\beta_{p_{1}}=0^{\circ}$ with maximum scaling factor. At this point we need not to apply any $r f$ field in the first period because the rotation around the $z$-axis in the rotating frame commutes with the spin Hamiltonian and no effect to the spin parts of the spin Hamiltonian happens during this rotation. As in the case of Tycko's trajectory, the mechanical rotation has to be synchronized with $\mathrm{rf}$ field, not only the phase but also the amplitude. Experimentally, the axes and rates of the spin rotation may be adjustable by change the offset and the amplitude of the rf field[71], like the magic angle spinning experiment in rotating frame by Lee and Goldberg in 1965[119]. 

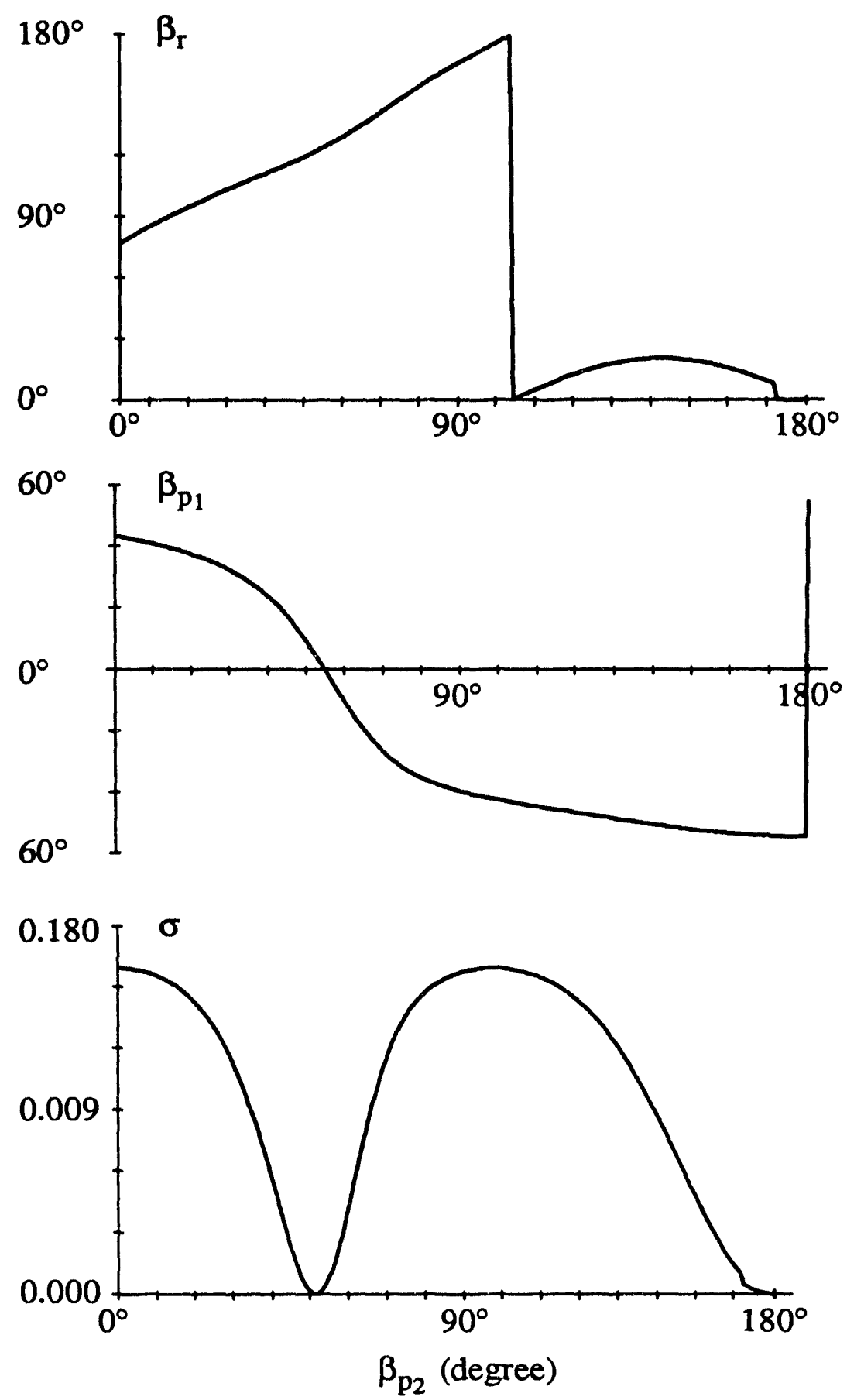

Figure 2.4: Exact solutions of $\beta_{r}, \beta_{p_{2}}$, and $\sigma$ as functions of the free variable $\beta_{p_{1}}$ in the $\left[0^{\circ}, 180^{\circ}\right]$ period. The zero order average Hamiltonian is a scalar operator scaled by a factor $\sigma$ from the untruncated internal spin Hamiltonian for each set of $\beta_{p_{1}}, \beta_{p_{2}}$, and $\beta_{r}$. 


\subsection{Optimized Solutions}

Three solutions are readily obtained from Eqs.(2.12) and (2.13). These solutions coincides with the trajectories used in dynamic angle spinning (DAS)[76, 78], double rotation (DOR) $[72,104]$, and dynamic angle hopping (DAH), which are used to eliminate the second order line broadenings of the central transitions in spin half integer quadrupolar nuclei. The similarity arises because the second order line broadenings are determined by both the second and the fourth rank spatial tensors of the first order average Hamiltonian in the rotating frame. The theory behind these trajectories are also similar, the main difference being that all the rotations applied in zero field NMR in high field experiment are performed in the coupled spatial-spin space. We briefly show here some of the results.

\subsubsection{Dynamic Angle Hopping (DAH)}

The first set of trajectories can be obtained by directly solving Eqs.(2.9) and (2.12) using discrete rotations. These solutions consist in a series of "hops" of the $z$-axis of the coupled spatial-spin space on paths given by two cones. On each cone, the solutions consist of five points separated by equal increments of $72^{\circ}$. Ten hops are therefore needed. Experimentally, these sudden changes can be implemented by hopping the quantization axes of the spin interaction in the coupled spatial-spin space simultaneously. Although the hoppings in spin space can be made quickly by applying rf pulses, it is much more difficult to mechanically hop the sample. Practically the hopping of the sample can be implemented by storing the magnetization along the external magnetic field (where the magnetization relaxes very slowly), changing the orientation of the sample in the laboratory frame, and then bringing back the magnetization to the xy-plane for further evolution. The problem involved in the storing-hopping experiment is that only one component of the magnetization in the $x y$-plane can be stored each times. It is therefore necessary to design the experiment 


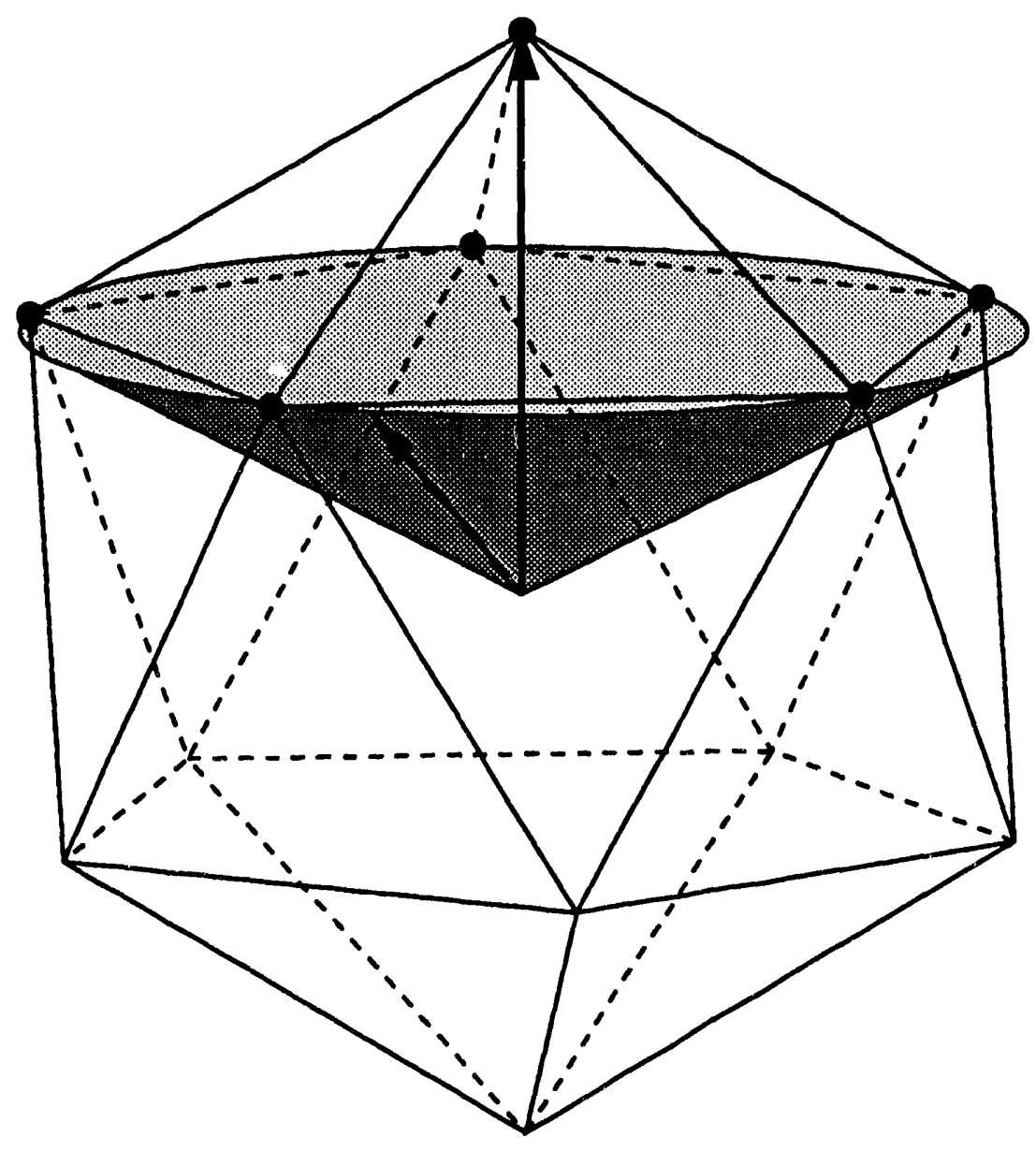

Figure 2.5: Dynamic angle hopping (DAH) trajectory:an icosahedron. The half apex angle of the first cone (indicated by the vertical arrow), $\theta^{(1)}=0^{\circ}$, and the half apex angle of the second cone (shaded cone), $\theta^{(2)}=63.43^{\circ}$ with respect to the external field. 
with minimum number of hops. The solution that minimizes the number of hops is a path defined by the vertices of an icosahedron (Figure (2.5)).

In this experiment which we call dynamic angle hopping (DAH), the half apex angle $\theta$ is zero for the first cone. For the second cone, the half apex angle $\theta$ is $63.43^{\circ}$ for the second cone. In a DAH cycle, the sample and the magnetization will hop through six vertices on the icosahedron with the the magnetization evolving for a time $\frac{\tau}{6}$ (where $\tau$ is the length of a cycle) under the spin Hamiltonian given by Eq.(2.6). When the sample and the magnetization have traced a closed path through all six vertices of the icosahedron, the average Hamiltonian has become a scalar. In this scalar, the coupling constants $\delta_{\lambda}$ of quadrupolar or dipolar interactions will be scaled down by a factor of five (that is, $\sigma=\frac{1}{5}$ ).

\subsubsection{Dynamic Angle Spinning (DAS) for Zero Field NMR in High Field}

Instead of using discrete rotations (hops), the $\mathrm{z}$-axis of the coupled spatial-spin space may travel continuously on the two cocentric cones that we have introduced. On the first cone spins will evolve for a time $t_{1}$ while on the second cone they evolve for a time $t_{2}$ under the Hamiltonian in Eq.(2.6). The half apex angles of the two cones depend on the ratio of the two evolution times $\frac{t_{1}}{t_{2}}$. If the ratio is 1 , the first half-apex angle $\beta_{1}=\theta^{(1)}=37.38^{\circ}$ and the second half-apex angle $\beta_{2}=\theta^{(2)}=79.19^{\circ}$. This trajectory possess a dodecahedral symmetry (see Figure (2.6)). Five vertices of the dodecahedron are located on the cone with half apex angle $\theta^{(1)}$, while other five vertices of the polyhedron are located on the cone with half apex angle $\theta^{(2)}$. We call this trajectory dynamic angle spinning (DAS)[76] for zero field NMR in high field, and, as in DAH, yields the coupling constants $\delta_{\lambda}$ scaled down by a factor of five (that is, $\left.\sigma=\frac{1}{5}\right)$. 


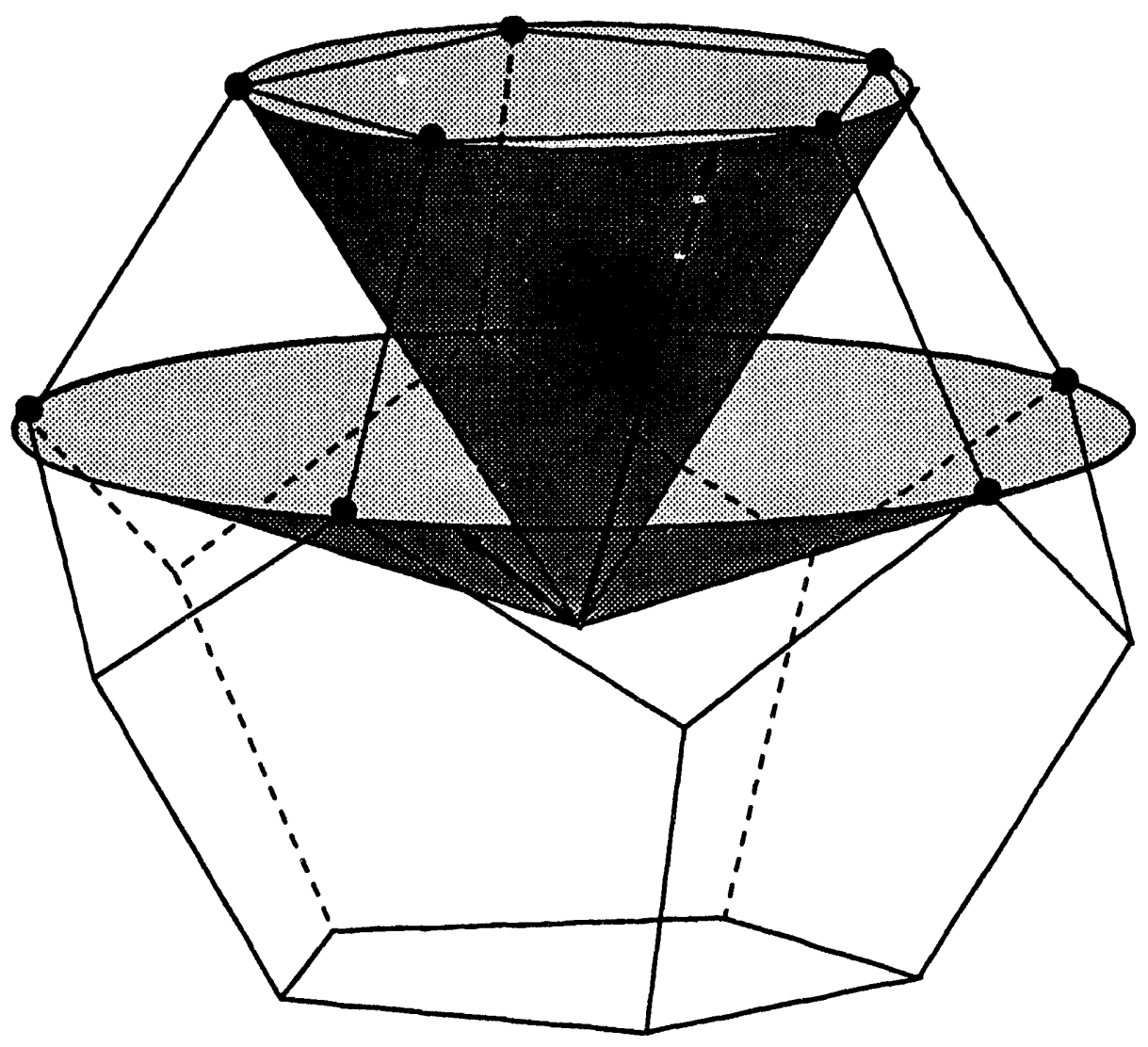

Figure 2.6: Dynamic angle spinning (DAS) trajectory:a dodecahedron. The half apex angle of the first cone (top shaded cone), $\theta^{(1)}=37.38^{\circ}$, and the half apex angle of the second cone (the bottom shaded cone), $\theta^{(2)}=79.19^{\circ}$ with respect to the external field. 


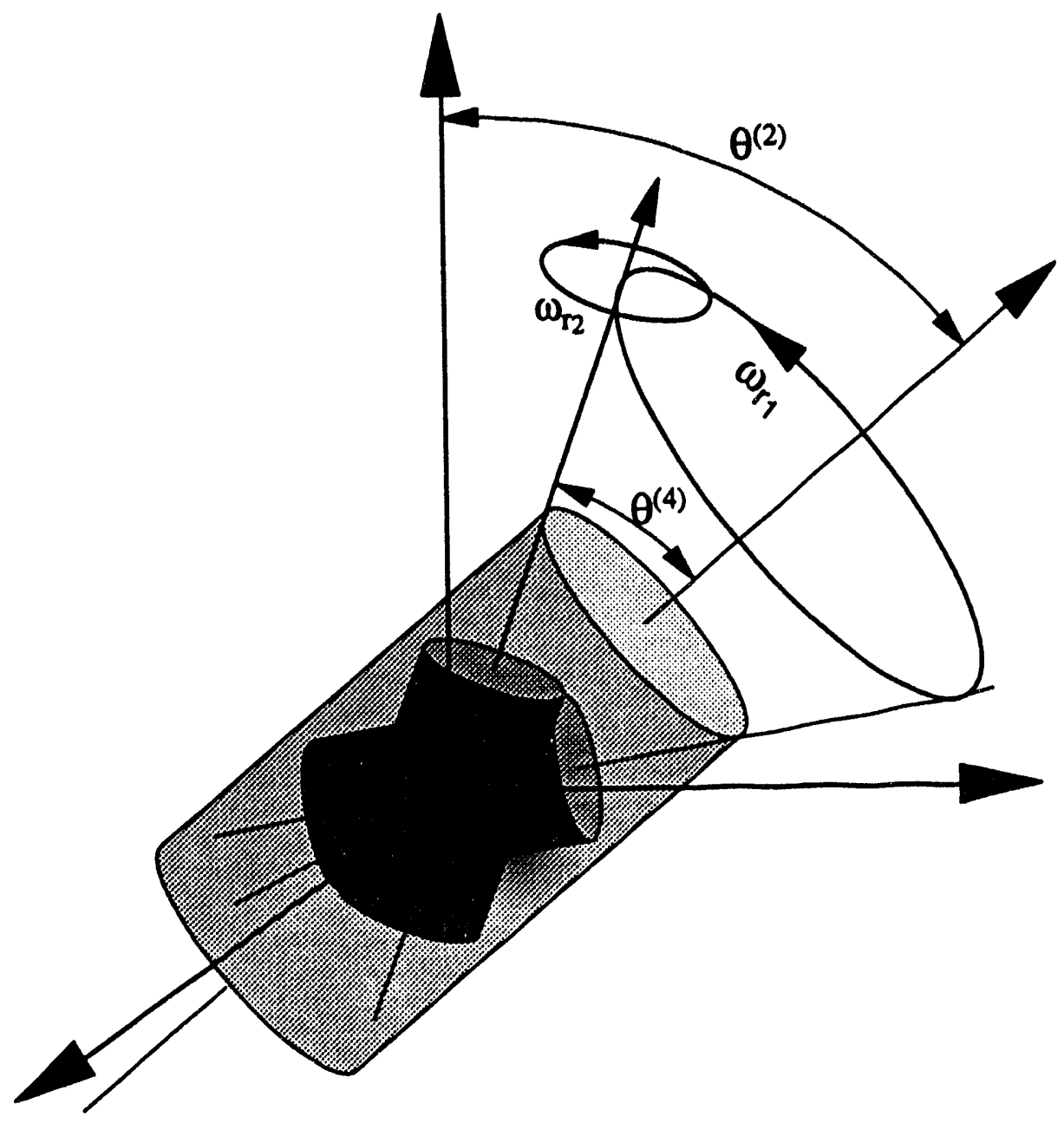

Figure 2.7: Double rotation (DOR) trajectory. The first rotation axis is tilted at $\theta^{(2)}=54.74^{\circ}$, the magic angle of the second order Legendre polynomial, with respect to the external field, and the second rotation axis is at $\theta^{(4)}=30.56^{\circ}$, one of magic angles of the fourth order Legendre polynomial, relative to the first rotation axis. 


\subsubsection{Double Rotation (DOR) for Zero Field NMR in High Field}

In the previous two trajectories, discrete rotations (hops) or continuous rotations applied to the $z$-axis of the coupled spatial-spin space occur at different times. According to Eq.(2.13), a zero field spin Hamiltonian can also be achieved by rotating the $z$-axis of the coupled spatial-spin space around two or more axes simultaneously A scalar Hamiltonian results when the one of the axes of the two continuous rotations is tilted at $\beta_{1}=\theta^{(2)}=54.74^{\circ}$, the 'magic' angle of the second order Legendre polynomial, with respect to the static magnetic field $H_{0}$, and the second axis of the rotations is at $\beta_{2}=\theta^{(4)}=30.56^{\circ}$, one of the 'magic' angles of the fourth order Legendre polynomial, relative to the first axis (see Fig. (2.7)). We call this trajectory the double rotation (DOR) [72] for zero field NMR in high field. The scaling factor of the coupling constants $\left(\delta_{\lambda}\right)$ with DOR trajectory is again $\sigma=\frac{1}{5}$. 


\section{Chapter 3}

\section{Second Order NMR Spectroscopy}

\subsection{Introduction}

The dispersion of local magnetic fields - the spread of resonant frequencies inherent even in the simplest systems - determines selectivity and resolution in NMR spectroscopy $[5,59]$. The local fields among a group of coupled nuclei, for example, may be rendered nonstationary owing to spin flip-flops, or spin diffusion, and the associated resonance can acquire a certain width as a result. This kind of linewidth usually is classified as a relaxation effect. Another source of frequency dispersion arises from the dependence of most spin interactions on the orientation of the external magnetic field relative to each nucleus. Thus both the spin and spacial dependence of the magnetic interactions must be addressed if one is to realize full spectroscopic control in NMR.

Local fields are manifested in different ways in solids and liquids, and spectroscopic methods need to be tailored accordingly. At one extreme there is the case of an isotropic liquid where, in the presence of rapid and random molecular reorientation, spatial anisotropy is averaged largely to zero. For sufficiently rapid tumbling, only the isotropic components of the spin interactions remain and so it becomes possible to achieve truly high resolution. In solids, however, restrictions on molecular motion prevent the spins from sampling a spherically symmetric set of orientations. Here, there are two general solutions: either remove the field altogether, and by so doing 
eliminate the very notion of directionality as in zero-field NMR [9] as mentioned in chapter 1 and 2. Alternatively, one can impose an artificial macroscopic motion on the spins to create the equivalent of a spherically symmetric environment. This latter option, that of supplying the motion externally, was inherent in the spin echo experiment[52] and its generalizations[53] in which the spin angular momenta are perturbed while leaving the molecules in place; and in the sample-spinning experiments of Anderson [54], in which the sample is rotated. Averaging in spin space has progressed from simple spin echoes to the prolonged trains introduced by Waugh and later workers for solids[55]. Averaging in spatial space was extended to solids by magic-angle spinning (MAS) $[56,57,58]$ which is a routine feature of solid state NMR. In this chapter we consider some new approaches that extend motional averaging in solid-state NMR, with particular emphasis on the NMR of systems governed by electric quadrupole interactions. These approaches are called dynamic angle spinning $(\mathrm{DAS})[73,76]$ and double rotation (DOR) $\left[72,104,78^{1}\right.$

\subsection{The First Order Average wimiltonian with a Quadrupolar Interaction}

We consider a spin system subjected to a quadrupolar interaction in the existence of a strong static external magnetic field. Its spin Hamiltonian in the laboratory frame $(\mathrm{LAB})$ is[19]

$$
\mathcal{H}=\omega_{0} I_{z}+\omega_{Q} \sum_{m=-2}^{2}(-1)^{m} A_{2-m} T_{2 m},
$$

where $\omega_{0}$ is Larmor frequency, and $\omega_{Q}$ is the quadrupolar coupling constant, given by

$$
\omega_{Q}=\frac{e^{2} q Q}{2 I(2 I+1) \hbar} .
$$

$A_{2, m}$ and $T_{2, m}$ are the elements of the second rank irreducible spatial and spin tensors respectively. The definition of $T_{2, m}$ for quadrupolar interaction is given by Eq.(1.33), 
and $A_{2, m}$ is

$$
A_{2, m}=\sum_{m^{\prime}} \mathcal{D}_{m^{\prime}, m}^{(2)}(\Omega) \rho_{2, m^{\prime}}
$$

where the $\rho_{2, m}$ are the principal values of the quadrupolar interaction tensor as listed in Eq.(1.4), the $\mathcal{D}_{m^{\prime}, m}^{(2)}(\Omega)$ are the components of Wigner rotation matrices, and $\Omega$ is the set of Euler angles for the transformation from the laboratory frame to the principle axis system (PAS) of the quadrupolar interaction.

The quadrupolar coupling constant appearing in Eq.(3.1) can be relate to the electrical-field-gradient (EFG) which is determined by the stereo-structure of the distribution of electrons around a particular nucleus in the molecule being studied. Measurement of the quadrupolar coupling constant therefore provides a method of obtaining the EFG informaticn. In order to extract this information, we need to know the evolution rules of the density matrix under the existing of the quadrupolar spin Hamiltonian. Following the routine procedure, the total spin Hamiltonian in Eq.(3.1) is transformed from the LAB frame into the rotating frame (or interactive representation).

$$
\begin{aligned}
\mathcal{H}(t) & =e^{2 \omega_{0} I_{z} t} \mathcal{H}_{Q} e^{-2 \omega_{0} I_{z} t} \\
& =\omega_{Q} \sum_{m=-2}^{2}(-1)^{m} A_{2-m} T_{2 m} e^{\imath m \omega_{0} t}
\end{aligned}
$$

This rotating frame spin Hamiltonian is modulated by the Larmor frequency and its harmonics. According to coherent average Hamiltonian theory[109, 115], the zero order and the first order average Hamiltonian of Eq.(3.4) are given by

$$
\begin{aligned}
\mathcal{H}^{(0)} & =\frac{1}{\tau_{c}} \int_{0}^{\tau_{c}} \mathcal{H}(t) d t \\
\mathcal{H}^{(1)} & =\frac{-i}{2 \tau_{c}} \int_{0}^{\tau_{c}} d t_{1} \int_{0}^{t_{1}} d t_{2}\left[\mathcal{H}\left(t_{1}\right), \mathcal{H}\left(t_{2}\right)\right]
\end{aligned}
$$

where $\tau_{c}=2 \pi / o m w g a_{0}$, the time length of a cycle Larmor oscillation.

For most internal spin interactions the zero order average Hamiltonian is already a very good approximation to the time dependent Hamiltonian given in Eq.(3.4) as 
the Zeeman term is usually large enough to truncate the internal spin interactions. However, for spin half integer qudrupolar nuclei, the quadrupolar coupling constants are usually so large that the Zeeman field cannot effectively truncate the quadrupolar interaction, similar to the situation discussed by VanderHart [60] in the low magnetic field for chemical shift anisotropic interaction. In such cases, the higher order effects have to be taken into account when transforming the total Hamiltonian from the $\mathrm{LAB}$ frame to the rotating frame. Inserting Eq.(3.4) into Eq.(3.5) yields the total average Hamiltonian up to the first order approximation in the rotating frame as

$$
\mathcal{H}=A_{20} T_{20}+\frac{\omega_{Q}^{2}}{2 \omega_{0}} \sum_{m \neq 0} \frac{1}{m}\left(A_{2 m} A_{2-m}\left[T_{2 m}, T_{2-m}\right]+A_{2 m} A_{20}\left[T_{2 m}, T_{20}\right]\right),
$$

where, and from now on, the same symbol as in the LAB frame is used to express the Hamiltonian in the rotating frame.

The first term in Eq.(3.6) is same as the first order correction given by perturbation theory. The second term includes two parts: a secular (commutes with the Zeeman Hamiltonian) and a non-secular (does not commute with the Zeeman Hamiltonain). As will be discussed later, the secular part in the second term corresponds to the second order correction of the eigenvalues. The presence of the non-secular term in the total Hamiltonian raises three questions. The first question is why there is a non-secular term in the first order average Hamiltonian. Next question is what contribution does the non-secular term make in the evolution of the spin system, and the final question is how to handle this non-secular term during the evolution of the density matrix. The answers of these questions are related to the original assumption of coherent average Hamiltonian theory. According to the fundamental rules in quantum mechanics[61], once the eigenstates and eigenvalues of the total Hamiltonian for a particular system are determined, all physical properties about the system are calculable. For example, the transition frequencies for a particular observable $\hat{O}$ are given by the differences of the eigenvalues and the intensity of a particular transition is determined by the eigenfunctions of the initial and final eigen- 
state, that is, $\left\langle\Psi|\hat{O}| \Psi^{\prime}\right\rangle$. In practice, to solve the eigenvalues and eigenstates of the total Hamiltonian for a complicated system usually is very difficulty and in many cases it is impossible. In perturbation theory, the eigenvalues and eigenfunctions of the total Hamiltonian are gradually approached by adding higher order corrections to the energy levels and the eigensfunctions of the total Hamiltonian. On the other hand, in average Hamiltonian theory, we first transform the total Hamiltonian from the Schrödinger representation to the interactive representation based on Zeeman Hamiltonian. The total Hamiltonian in the interactive representation becomes a periodic function of time with period $\tau_{c}=2 \pi /$ omega $a_{0}$. We then assume that we are only interested in the behavior of the density matrix at times $n \tau_{c}$ during the evolution. The value of the density matrix at these periodic points are approximately given by an average Hamiltonian which is time independent and discards the exact evolution during a cycle. In other words, the average Hamiltonian theory[109, 115] gives a stroboscopic description of the time evolution of the density matrix under the total Hamiltonian for a system. The average Hamiltonian can be approached by adding the higher order corrections which are functions of multiple time integrals over the products of the time dependent Hamiltonian, and includes all the contributions from fast to slow variation of the density matrix. Thus the average Hamiltonian in general is not diagonal. If we only interested in the slowest variation of the density matrix in the $n$-th order correction, we simply drop the non-secular part. Such a treatment of the average Hamiltonian corresponds to secular averaging Hamiltonian theory $[19,63,64,65]$.

We now go back to continue the derivation of the first order average Hamiltonian. For half integer spin $\left(I>\frac{1}{2}\right)$ only the central transition $\left(-\frac{1}{2} \leftrightarrow \frac{1}{2}\right)$ can be observed in the NMR experiments. Since the first order perturbation term in the Hamiltonian does not alter this transition frequency it can be ignored, and therefore from now on we need only focus on the secular part of the first order average Hamiltonian. Using 
the product properties of two irreducible tensors $[20,21]$, the secular part of the first order average Hamiltonian may be rewritten as:

$$
\mathcal{H}^{(1)}=\sum_{l^{\prime}=1,3} \sum_{l=0}^{4} \sigma_{l l^{\prime}} A_{l 0} T_{l^{\prime} 0}
$$

The $A_{l 0}, T_{l 0}$ terms in Eq (3.7) are spatial and spin parts of $l$-th irreducible tensors arising from the direct product of two second order irreducible tensors respectively; its explicit expression can be found in Tables (1.5) and (1.6), and $\sigma_{l l^{\prime}}$ represent the coupling constants of the second order quadrupolar interaction arising from higher order approximation, and are given by

$$
\begin{aligned}
\sigma_{l l^{\prime}}= & (-1)^{l^{\prime}} W\left(2,2, I, I ; l^{\prime}, I\right) \frac{5 \omega_{Q}^{2}<I\left|T_{2}\right| I>^{2}}{\omega_{0}<I\left|T_{l^{\prime}}\right| I>} \\
& \sum_{m \neq 0} \frac{1}{m} C(2,2, l, m,-m, 0) C\left(2,2, l^{\prime}, m,-m, 0\right),
\end{aligned}
$$

where

$$
<I\left|T_{l}\right| I>=l !\left[\frac{(2 I+l+1) !}{2^{l}(2 I+1)(2 l) !(2 I-l) !}\right]^{2}
$$

and

$$
<I m\left|T_{l q}\right| I m^{\prime}>=<I\left|T_{l}\right| I>\sqrt{\frac{2 l+1}{2 I+1}} C\left(I, l, I ; m, q, m^{\prime}\right) .
$$

In Eq. (3.8), $C\left(l_{1}, l_{2}, l ; m_{1}, m_{2}, m\right)$ are Clebsch-Gordan coefficients, $W\left(2,2, I, I ; l^{\prime}, I\right)$ are $6-j$ symbols, $I$ is spin value, and $l, l^{\prime}$ are the tensor ranks. Values of $\left\langle I\left|T_{l}\right| I\right\rangle$, $W\left(2,2, I, I ; l^{\prime}, I\right)$, and $\sigma_{l l^{\prime}}$ for spin $\frac{3}{2}, \frac{5}{2}$, and $\frac{7}{2}$ are listed in Tables (3.1), (3.2), and (3.3) respectively.

Because the quadrupolar coupling tensor is a real symmetric operator, the odd rank irreducible tensors $A_{10}$ are zero. Thus only the $A_{00}, A_{20}$, and $A_{40}$ terms remain in Eq. (3.8), among which only the $A_{20}$ and $A_{40}$ depend on orientations. Their principal values are given by

$$
\left\{\begin{array}{l}
\rho_{00}=\frac{\delta^{2}\left(3+\eta^{2}\right)}{2 \sqrt{5}} \\
\rho_{20}=-\frac{\delta^{2}\left(3-\eta^{2}\right)}{\sqrt{14}}, \rho_{2 \pm 1}=0, \rho_{2 \pm 2}=\sqrt{\frac{3}{7}} \eta \delta^{2} \\
\rho_{40}=\frac{\delta^{2}\left(9+\frac{\eta^{2}}{2}\right)}{\sqrt{70}}, \rho_{4 \pm 1}=0, \rho_{4 \pm 2}=\frac{3}{2 \sqrt{7}} \eta \delta^{2}, \rho_{4 \pm 3}=0, \rho_{4 \pm 4}=\frac{1}{4}(\eta \delta)^{2}
\end{array}\right.
$$


Table 3.1: Values of $\left\langle I\left|T_{l}\right| I\right\rangle$.

\begin{tabular}{|c|c|c|c|}
\hline & $l=1$ & $l=2$ & $l=3$ \\
\hline$I=\frac{3}{2}$ & $\sqrt{5}$ & $\sqrt{6}$ & $3 \sqrt{2}$ \\
\hline$I=\frac{5}{2}$ & $\sqrt{\frac{35}{2}}$ & $2 \sqrt{14}$ & $9 \sqrt{2}$ \\
\hline$I=\frac{7}{2}$ & $\sqrt{42}$ & $6 \sqrt{7}$ & $3 \sqrt{165}$ \\
\hline
\end{tabular}

Table 3.2: Value of $W(2,2, I, I, l, I)$ coefficients.

\begin{tabular}{|l|c|c|c|}
\hline \hline & $I=\frac{3}{2}$ & $I=\frac{5}{2}$ & $I=\frac{7}{2}$ \\
\hline$l=1$ & $-\frac{1}{5 \sqrt{2}}$ & $-\frac{1}{5 \sqrt{7}}$ & $-\frac{1}{2 \sqrt{105}}$ \\
\hline$l=3$ & $\frac{1}{5 \sqrt{2}}$ & $\frac{9}{70 \sqrt{2}}$ & $\frac{1}{14} \sqrt{\frac{11}{15}}$ \\
\hline
\end{tabular}

where $\delta$ is the anisotropy (for our case it is always set to one) and $\eta$ is the asymmetry parameter of the quadrupolar interaction. The orientation dependence of the spatial irreducible tensor $A_{l, m}$ can therefore be explicitly represented by using the Wigner rotation matrices $\mathcal{D}_{m^{\prime}, m}^{(l)}(\alpha, \beta, \gamma)$ and the principal values as

$$
A_{l m}=\sum_{m^{\prime}=-l}^{l} \mathcal{D}_{m^{\prime}, m}^{(l)}(\alpha, \beta \gamma) \rho_{l m^{\prime}}
$$

where $\alpha, \beta, \gamma$ are the three Euler angles relating the laboratory frame and the principle axis system of the quadrupolar interaction.

Table 3.3: Values of $\sigma_{l l^{\prime}}$.

\begin{tabular}{|c|c|c|c|c|}
\hline \hline$l l^{\prime}$ & 21 & 23 & 41 & 43 \\
\hline$I=\frac{3}{2}$ & $-\frac{6}{5 \sqrt{14}}$ & $\frac{6}{\sqrt{35}}$ & $\frac{18}{5 \sqrt{70}}$ & $\frac{17}{5 \sqrt{7}}$ \\
\hline$I=\frac{5}{2}$ & $-\frac{16}{5 \sqrt{14}}$ & $\frac{6}{\sqrt{35}}$ & $\frac{48}{5 \sqrt{70}}$ & $\frac{17}{5 \sqrt{7}}$ \\
\hline$I=\frac{7}{2}$ & $-\frac{6}{\sqrt{14}}$ & $\frac{6}{\sqrt{35}}$ & $\frac{18}{\sqrt{70}}$ & $\frac{17}{5 \sqrt{7}}$ \\
\hline
\end{tabular}




\subsection{Static Powder Lineshapes}

Second order frequency shifts of the central transitions in half integer spin quadrupolar nuclei possessing a quadrupolar interaction can be calculated by use of Eq. (3.7) and Tables (3.1-3.3). These frequency shifts are orientation dependent. Such an orientation dependence results in characteristic powder patterns when dealing with polycrystalline or amorphous samples. These powder patterns are in general different from the ones originating from first order contribution of the spin interactions [19]. In this section we describe these properties of the powder lineshapes under various circumstances.

We start by presenting an alternative method for evaluating the frequency shifts of the central transition in the presence of quadrupolar interactions by use of the second order perturbation theory in Eq.(3.1). From the properties of the spin operators ( $I_{x}$, $I_{y}$, and $I_{z}$ ), the matrix representations of the spin operators $I_{+}, I_{-}$, and $I_{0}$ can be obtained in the Zeeman basis functions.

$$
\begin{aligned}
<m\left|I_{0}\right| m^{\prime}> & =<m\left|I_{z}\right| m>=m \delta_{m, m^{\prime}} \\
\left.<m\left|I_{ \pm}\right| m^{\prime}\right\rangle & =\frac{1}{2}<m\left|I_{x} \pm \imath I_{y}\right| m>=\sqrt{(I \mp m)(I \pm m+1)} \delta_{m, m^{\prime} \pm 1}
\end{aligned}
$$

where $m$ runs from $--I$ to $I, I$ being the spin value. The relationships between the irreducible spin tensors and the spherical spin operators are given by

$$
\begin{aligned}
T_{1,0} & =I_{0} \\
T_{1, \pm 1} & =\mp \frac{1}{\sqrt{2}} I_{ \pm} .
\end{aligned}
$$

Inserting Eqs.(3.13) and (3.14) into Eqs. (1.33) and (1.34) yields the matrix representations of the components of the second rank irreducible spin tensor as

$$
\begin{aligned}
<m\left|T_{2,0}\right| m^{\prime}> & =\frac{1}{\sqrt{6}}\left[3 m^{2}-I(I+1)\right] \delta_{m, m^{\prime}} \\
<m\left|T_{2, \pm 1}\right| m^{\prime}> & =\mp \frac{1}{2}(2 m \pm 1) \sqrt{(I \mp m)(I \pm m+1)} \delta_{m, m^{\prime} \pm 1}
\end{aligned}
$$




$$
<m\left|T_{2, \pm 2}\right| m^{\prime}>=\frac{1}{2} \sqrt{(I \mp m)(I \pm m+1)(I \mp m-1)(I \pm m+2)} \delta_{m, m^{\prime} \pm 2} .
$$

The matrix representation of the total spin Hamiltonian can be obtained by inserting Eq.(3.15) into Eq.(3.1). In this matrix representation of the spin Hamiltonian in the Zeeman basis functions, only the elements of five central diagonals are different from zero. Using perturbation theory, the second order corrections of the eigenvalues of the spin states $m= \pm \frac{1}{2}$ are

$$
\begin{aligned}
& E_{\frac{1}{2}}^{(2)}=-\frac{\left|\mathcal{H}_{\frac{1}{2}, \frac{5}{2}}\right|^{2}}{2 \omega_{0}}-\frac{\left|\mathcal{H}_{\frac{1}{2}, \frac{3}{2}}\right|^{2}}{\omega_{0}}+\frac{\left|\mathcal{H}_{\frac{1}{2},-\frac{1}{2}}\right|^{2}}{\omega_{0}}+\frac{\left|\mathcal{H}_{\frac{1}{2},-\frac{3}{2}}\right|^{2}}{2 \omega_{0}} \\
& E_{-\frac{1}{2}}^{(2)}=-\frac{\left|\mathcal{H}_{-\frac{1}{2}, \frac{3}{2}}\right|^{2}}{2 \omega_{0}}-\frac{\left|\mathcal{H}_{-\frac{1}{2}, \frac{1}{2}}\right|^{2}}{\omega_{0}}+\frac{\left|\mathcal{H}_{-\frac{1}{2},-\frac{3}{2}}\right|^{2}}{\omega_{0}}+\frac{\left|\mathcal{H}_{-\frac{1}{2},-\frac{5}{2}}\right|^{2}}{2 \omega_{0}}
\end{aligned}
$$

and therefore the second order frequency shifts of the central transitions are

$$
\omega_{\frac{1}{2} \leftrightarrow-\frac{1}{2}}^{(2)}=\frac{\left|\mathcal{H}_{\frac{1}{2},-\frac{3}{2}}\right|^{2}}{2 \omega_{0}}+\frac{\left|\mathcal{H}_{-\frac{1}{2}, \frac{3}{2}}\right|^{2}}{2 \omega_{0}}-\frac{\left|\mathcal{H}_{\frac{1}{2}, \frac{3}{2}}\right|^{2}}{\omega_{0}}-\frac{\left|\mathcal{H}_{-\frac{1}{2},-\frac{3}{2}}\right|^{2}}{\omega_{0}}-\frac{\left|\mathcal{H}_{\frac{1}{2}, \frac{5}{2}}\right|^{2}}{2 \omega_{0}}-\frac{\left|\mathcal{H}_{-\frac{1}{2},-\frac{5}{2}}\right|^{2}}{2 \omega_{0}}
$$

As can be seen from this expression in the matrix representation of the spin Hamiltonian the first off-diagonal column is related to $A_{2, \pm 1}$ and the second offdiagonal column arises from $A_{2, \pm 2}$.

After some simple algebra, the second order frequency shifts of central transitions owing to the quadrupolar interactions can be written as

$$
\omega_{\frac{1}{2} \rightarrow-\frac{1}{2}}^{(2)}=\frac{\omega_{\Im}^{2}}{\omega_{0}}\left(I-\frac{1}{2}\right)\left(I+\frac{3}{2}\right)\left[\left|A_{2,2}\right|^{2}-2\left|A_{2,1}\right|^{2}\right] .
$$

From the properties of the irreducible tensor again we have that

$$
A_{l, m}^{\dagger}=(-1)^{m} A_{l,-m}
$$

Inserting Eq.(3.19) into Eq.(3.18), and then applying Eq.(3.4) yields the total second order frequency shifts of central transitions expressing in the basis set of irreducible tensors from rank zero to four,

$$
\omega_{\frac{1}{2} \leftrightarrow-\frac{1}{2}}^{(2)}=\frac{\omega_{Q}^{2}}{\omega_{0}}\left(I-\frac{1}{2}\right)\left(I+\frac{3}{2}\right) \sum_{l=0,2,4}[C(2,2, l, 2,-2)+2 C(2,2, l, 1,-1)] A_{l, 0},
$$


where $C\left(l_{1}, l_{2}, l, m_{1}, m_{2}\right)$ are the Clebsch-Gordan coefficients and $A_{l, 0}$ are the coupled tensor elements. The reason of the explosion of odd numbers of index $l$ in Eq.(3.20) is that the quadrupolar interaction is symmetric and the perturbation approximation does not change this symmetry property. Among the three coupled tensors appearing in Eq.(3.20), $A_{0,0}$ is the only scalar under rotation operations to the sample. This term creates an isotropic frequency shift to the peak in NMR spectra. Applying Eq.(3.12) and the principal values of the coupled sensors $A_{l}$ given by Eq.(3.11) into Eq.(3.20), we can derive this second order isotropic shift as

$$
\omega_{i s o}^{(2)}=-\frac{\left(3+\eta^{2}\right) \omega_{Q}^{2}}{10 \omega_{0}}\left(I-\frac{1}{2}\right)\left(I+\frac{3}{2}\right) .
$$

As can be seen, the second order isotropic shift of the quadrupolar interaction is proportional to the square of the quadrupolar coupling constant and inversely proportional to the Larmor frequency. Therefore as higher magnetic field are applied, the second order isotropic quadrupolar shifts become less important.

The remaining terms in Eq.(3.20) are orientation dependent. The lineshape observed in the solid-state NMR arising from a powder sample is therefore determined by both the second rank and fourth rank tensors simultaneously. This static lineshape can be obtained by performing a powder average in the frequency domain of the transition frequencies determined by Eq.(3.20), weighted by the transition probabilities $P=\sin \beta$. Figure (3.1) shows a set of simulated static powder patterns varying with respect to the asymmetry parameters $\eta$. As can be seen from Eq.(3.20), the ratio of the second rank tensor and fourth rank tensor is independent of the spin values. The lineshape therefore is also independent of the spin values, and thus the lineshapes shown in Figure (3.1) can be expected for the central transitions of any half integer spin nuclei.

Practically important in the analysis of powder patterns is the positions of the singularities appearing in the powder lineshapes, and these singularities can be de- 


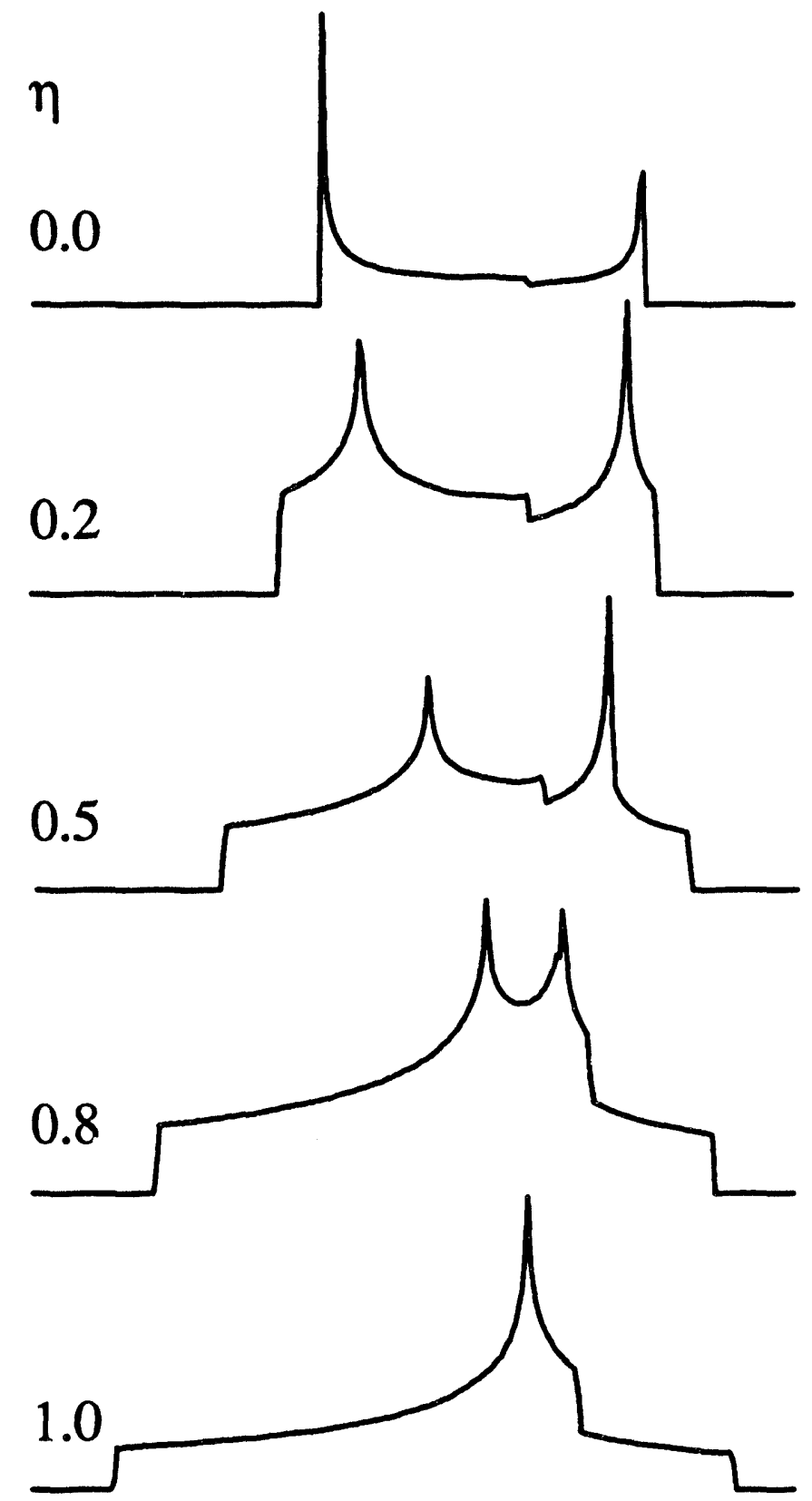

Figure 3.1: Variation of simulated powder patterns of the central transitions for half integer spin nuclei subjected to quadrupolar interactions with different asymmetry parameters $\eta$. 
rived from the relationships $[19,115]$

$$
\frac{\partial}{\partial \beta} \omega_{\frac{1}{2} \leftrightarrow-\frac{1}{2}}^{(2)}=0, \quad \text { and } \frac{\partial}{\partial \alpha} \omega_{\frac{1}{2} \leftrightarrow-\frac{1}{2}}^{(2)}=0 .
$$

The solution of Eq.(3.22) can be found $u$ ing the explicit representation of the second order shift $\omega_{\frac{1}{2} \leftrightarrow-\frac{1}{2}}^{(2)}$ in terms of trigonometric functions. By use of Eqs. (3.11), (3.12) and the representation of Wigner rotation matrices, this second order shift can be written as

$$
\begin{aligned}
\omega_{\frac{1}{2} \hookleftarrow-\frac{1}{2}}^{(2)} & =\omega_{Q}^{(2)}\left[-\frac{3-\eta^{2}}{7}\left(3 \cos ^{2} \beta-1\right)+\frac{6}{7} \eta \sin ^{2} \beta \cos 2 \alpha+\frac{9}{32} \eta^{2} \sin ^{4} \beta \cos 4 \alpha\right. \\
& \left.+\frac{9\left(9+\frac{1}{2} \eta^{2}\right)}{560}\left(3-30 \cos ^{2} \beta+35 \cos ^{4} \beta\right)+\frac{27}{56} \eta\left(7 \cos ^{2} \beta-1\right) \sin ^{2} \beta \cos 2 \alpha\right],
\end{aligned}
$$

where $\omega_{Q}^{(2)}$ can be thought fictitiously as a second order quadrupolar coupling constant with the definition of

$$
\omega_{Q}^{(2)}=\frac{\omega_{Q}^{2}}{\omega_{0}}\left(I-\frac{1}{2}\right)\left(I+\frac{3}{2}\right)
$$

Inserting $\mathrm{Eq}(3.23)$ into Eq.(3.22) yields the singularities of a powder lineshape, listed in Table (3.4). As will be discussed later, the singularities listed in this table are also for the powder lineshapes under variable angle sample spinning (VASS). The second order Legendre polynomial $P_{2}$ and the fourth order Legendre polynomial $P_{4}$ are used to represent the scaling factors of the lineshapes under VASS. For static powder lineshapes, both of $P_{2}$ and $P_{4}$ are simply set to unity. One of the six singularities only shows up when $\eta \geq\left|\frac{9 P_{4}-16 P_{2}}{21 P_{4}}\right|=\frac{1}{3}$. When the asymmetry parameter is zero, the second and third singularities will degenerate.

Figure (3.2) shows the frequency variation of the singularities in the powder lineshapes arising from second order effects as a function of the asymmetry parameter. In the figure, two of the six lines represent the sharp peaks of the lineshape, $\omega_{P_{1}}=\frac{1}{80}\left(69-30 \eta+13 \eta^{2}\right) \omega_{Q}^{(2)}$ for $0 \leq \eta \leq \frac{1}{3}$, or $\omega_{P_{1}}=\frac{2}{5}\left(2-\eta^{2}\right)$ for $\frac{1}{3} \leq \eta \leq 1$, and $\omega_{P_{2}}=\frac{1}{7}\left(-7+10 \eta+\eta^{2}\right) \omega_{Q}^{(2)}$ for $0 \leq \eta \leq 1$. other two are the shoulders, 


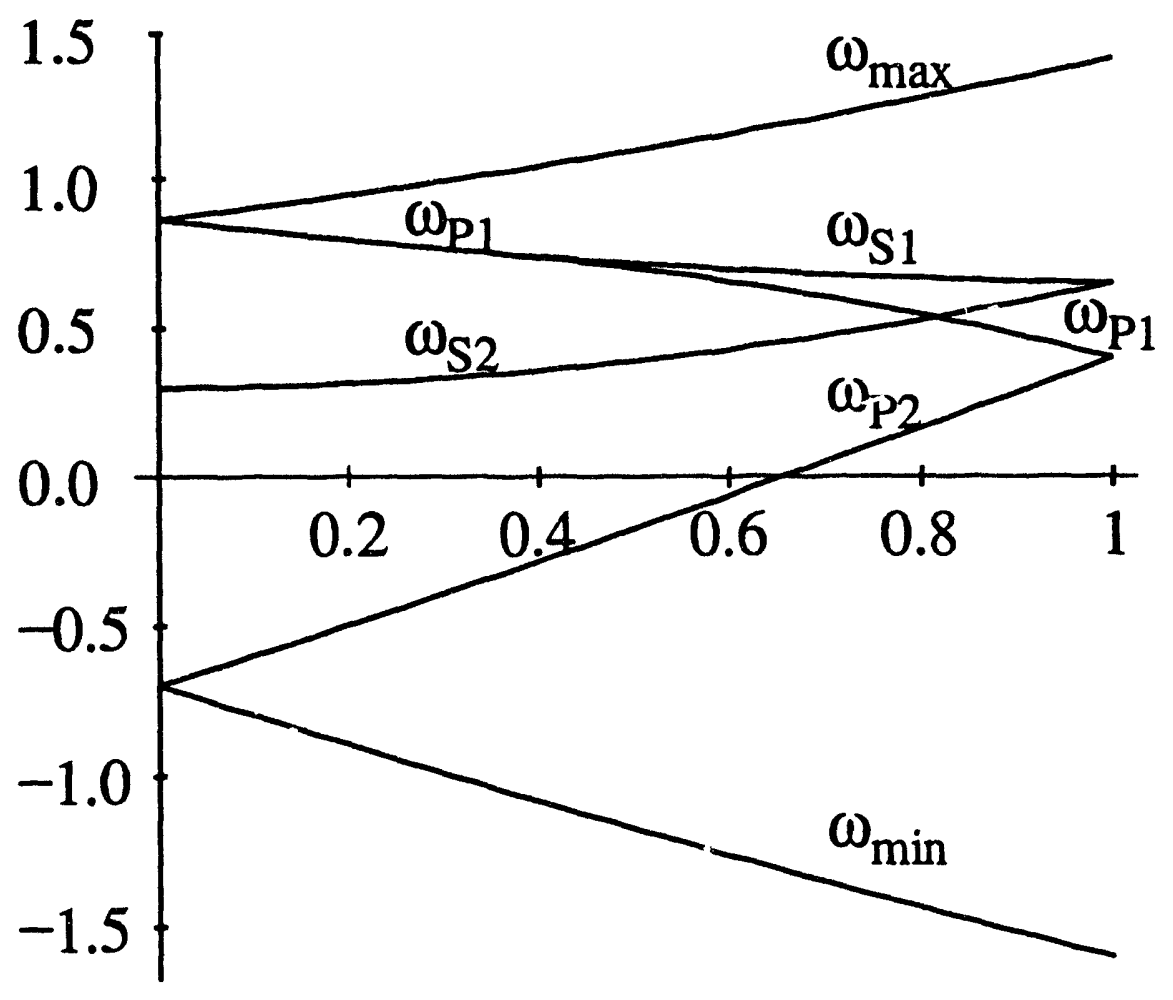

Figure 3.2: Frequency Variation of the singularities otserved in powder lineshapes arising from second order effects of the quadrupolar interactions in half integer spin nuclear systems as the symmetry parameter $\eta$ changes. 
Table 3.4: Singularities appearing in second order powder patterns

\begin{tabular}{|c|c|c|}
\hline$\beta$ & $\alpha$ & {$\left[\omega_{-\frac{1}{2} \rightarrow \frac{1}{2}}^{(2)}-\omega_{i s o}\right] / \omega_{Q}^{(2)}$} \\
\hline 0 & any & $\frac{162 P_{4}-120 P_{2}+\left(40 P_{2}+9 P_{4}\right) \eta^{2}}{140}$ \\
\hline$\frac{\pi}{2}$ & 0 & $\frac{243 P_{4}+240 P_{2}-\left(480 P_{2}-270 P_{4}\right) \eta-\left(80 P_{2}-171 P_{4}\right) \eta^{2}}{256}$ \\
\hline$\frac{\pi}{2}$ & $\frac{\pi}{2}$ & $\frac{243 P_{4}+240 P_{2}+\left(480 P_{2}-270 P_{4}\right) \eta-\left(80 P_{2}-171 P_{4}\right) \eta^{2}}{256}$ \\
\hline$\frac{\pi}{2}$ & $\frac{1}{2} \cos ^{-1}\left[\frac{9 P_{4}-16 P_{2}}{21 \eta P_{4}}\right]$ & $-\frac{80 P_{2}^{2}-195 P_{2} P_{4}-81 P_{4}^{2}+\left(35 P_{2} P_{4}+63 P_{4}^{2}\right) \eta^{2}}{245}$ \\
\hline $\cos ^{-1} \pm \sqrt{\frac{a_{+} P_{4}-b_{+} P_{2}}{c_{4}+P_{4}}}$ & 0 & $-\frac{40 P_{2}^{2}+60 P_{2} P_{4}+243 P_{4}^{2}+\left(40 P_{2}^{2}-80 P_{2} P_{4}-9 P_{4}^{2}\right) \eta^{2}}{490}$ \\
& & $+\frac{\left(80 P_{2}^{2}-300 P_{2} P_{4}-270 P_{4}^{2}\right) \eta}{490}$ \\
\hline $\cos ^{-1} \pm \sqrt{\frac{a_{-} P_{4}-b_{-} P_{2}}{c-P_{4}}}$ & $\frac{\pi}{2}$ & $-\frac{40 P_{2}^{2}+60 P_{2} P_{4}+243 P_{4}^{2}+\left(40 P_{2}^{2}-80 P_{2} P_{4}-9 P_{4}^{2}\right) \eta^{2}}{490}$ \\
& & $-\frac{\left(80 P_{2}^{2}-300 P_{2} P_{4}-270 P_{4}^{2}\right) \eta}{490}$ \\
\hline \hline
\end{tabular}

where $a_{ \pm}= \pm 216 \eta-45 \eta^{2}-243, b_{ \pm}=24\left(3-\eta^{2} \pm 2 \eta\right)$, and $c_{ \pm}=7( \pm 45 \eta-$ $\left.9 \eta^{2}-81\right) . P_{2}$ and $P_{4}$ are the second and fourth order Legendre polynomials.

$\omega_{S_{1}}=\frac{1}{80}\left(69-30 \eta+13 \eta^{2}\right) \omega_{Q}^{(2)}$ for $0 \leq \eta \leq \frac{1}{3}$ and $\omega_{S_{2}}=\frac{1}{20}\left(6+7 \eta^{2}\right)$ for $0 \leq \eta \leq 1$ while the flanks of the powder lineshapes are given by maximum and minimum frequencies, $\omega_{\min }=\frac{1}{10}\left(-7-10 \eta+\eta^{2}\right)$ and $\omega_{\max }=\frac{1}{80}\left(69+30 \eta+13 \eta^{2}\right)$ for $0 \leq \eta \leq 1$.

\subsection{Powder Lineshapes under VASS}

To discuss the behavior of second order effects of the quadrupolar interaction upon rotation along an axis forming a variable angle with respect to the external field, which is called variable angle sample spinning (VASS) $[103,88]$, we have to introduce a relationship between the laboratory frame, the sample-fixed coordinates frame (SFC), and the principle axis system (PAS) in Figure (3.3). Because the sample rotation only affects the spatial part of the spin Hamiltonian, implying that the spin Hamiltonian commutes with itself at all times. Henceforth, the eigenvectors are not mixed with each other, and only the eigenvalues of the spin Hamiltonian oscillate periodically during the sample spinning. The time dependent resonance frequencies 


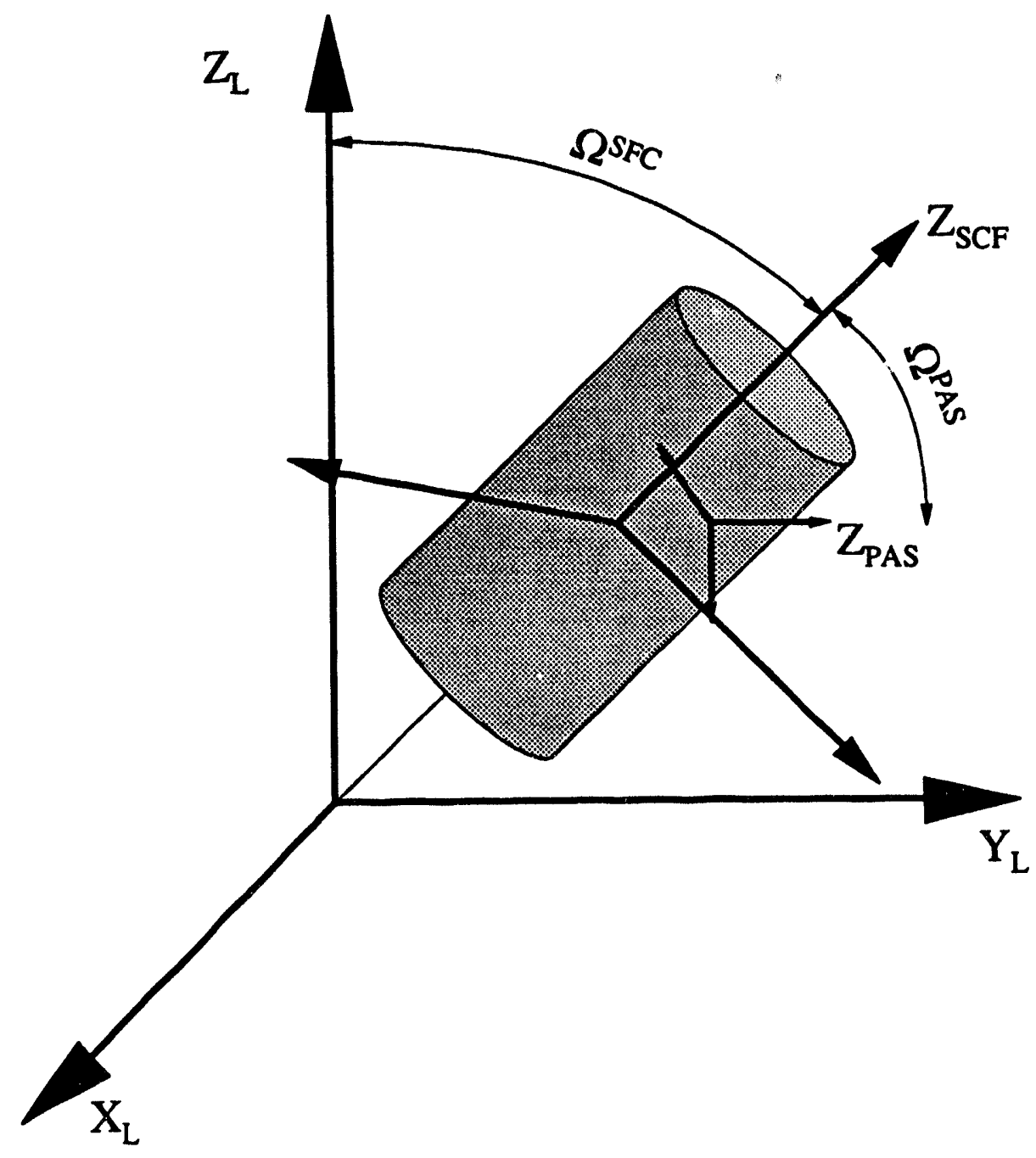

Figure 3.3: Two successive sets of Euler angles, $\Omega^{S F C}$ and $\Omega^{P A S}$, determine the direction of the magnetic field relative to the principle axis system at each nuclear site. 


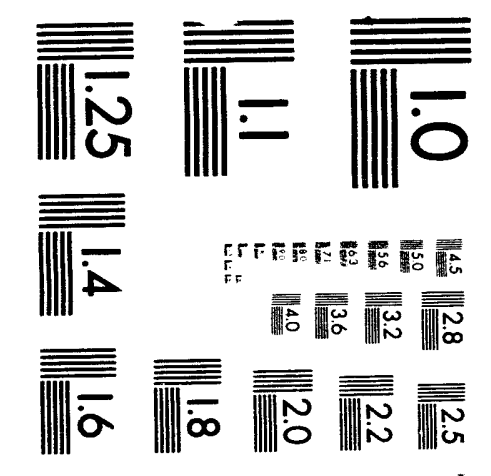



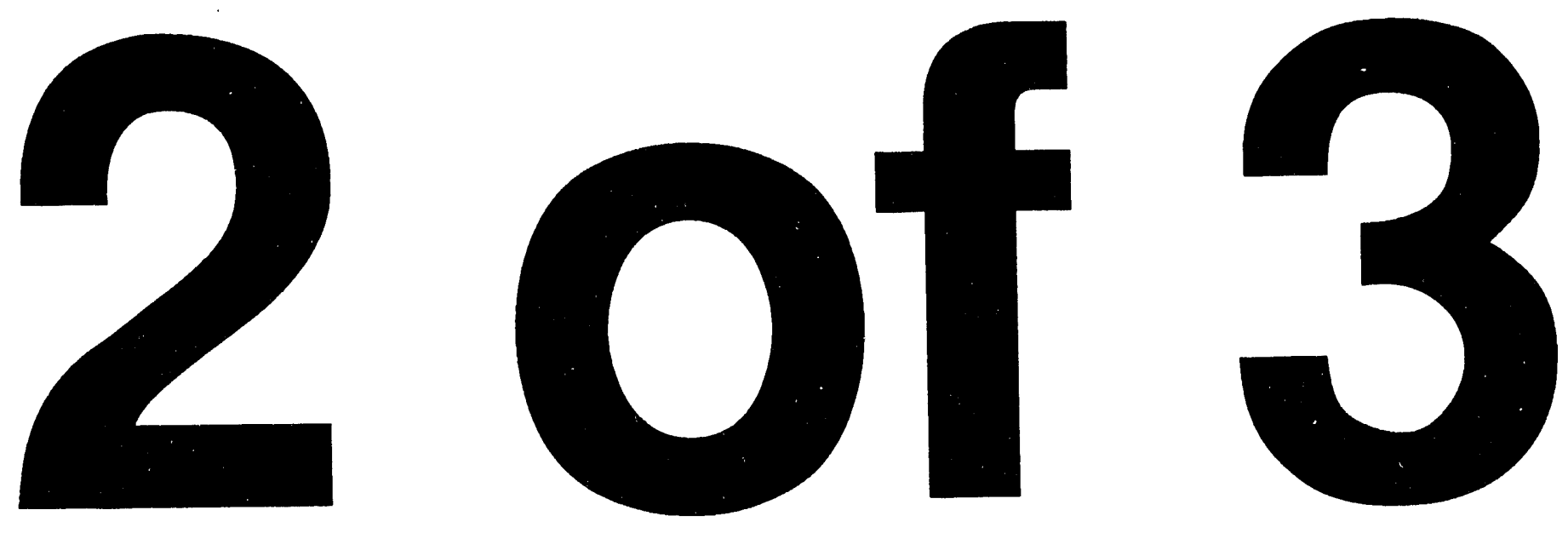
of the nuclear spin resulting from this process can be written as

$$
\omega_{\frac{1}{2} \leftrightarrow-\frac{1}{2}}^{(2)}=\sum_{l=0,2,4} \sum_{m} C_{l} \mathcal{D}_{m, 0}^{(l)}\left(\Omega^{S F C}(t)\right) A_{l, m}
$$

where

$$
C_{l}=\omega_{Q}^{(2)}[C(2,2, l, 2,-2)+2 C(2,2, l, 1,-1)] .
$$

Under the fast spinning condition, where the spinning speed is much larger than the amplitude of the second order quadrupolar interaction, the fast oscillating time dependent terms are averaged out in the time scale of experimental measurement. Under these circumstances, powder lineshapes are only determined by the time independent part in Eq.(3.25). Figure (3.4) shows the variation of the simulated powder lineshapes with different asymmetry parameters $\eta$ of the quadrupolar interaction, and different angles of the sample spinning axis with respect to the external field.

The singularities of the powder patterns are given in Table (3.4), where the second and fourth order Legendre polynomials, $P_{2}(\cos \theta)$ and $P_{4}(\cos \theta)$, depend on the angle $\theta$ of the rotation axis with respect to external field. When $\theta=30.56^{\circ}$, or $\theta=70.12^{\circ}$, the magic angles of the fourth order Legendre polynomial, the equations in last three rows in Table (3.4) are no longer valid because the right side of the equations tends to diverge at $P_{4}=0$. Therefore, near the region of the magic angles of the fourth order Legendre polynomial only three singularities appear and the total powder lineshapes become similar to the ones arising from the chemical shift anisotropy effect (see Figure 3.4). The boundary condition for the singularities can be actually found from the valid solutions of $\alpha$ and $\beta$ in Table (3.4). These results are represented graphically in Figure (3.5). As can be seen that there are two gaps around the magic angles of the fourth order Legendre polynomial, whose widths depend on the asymmetry parameter. The fourth singularity does no longer exist when the angles of the rotation axis are larger than $62^{\circ}$.

Figure (3.6) shows three-dimensional graphs of the frequency variations of the singularities observed in second order powder lineshapes for different asymmetry 


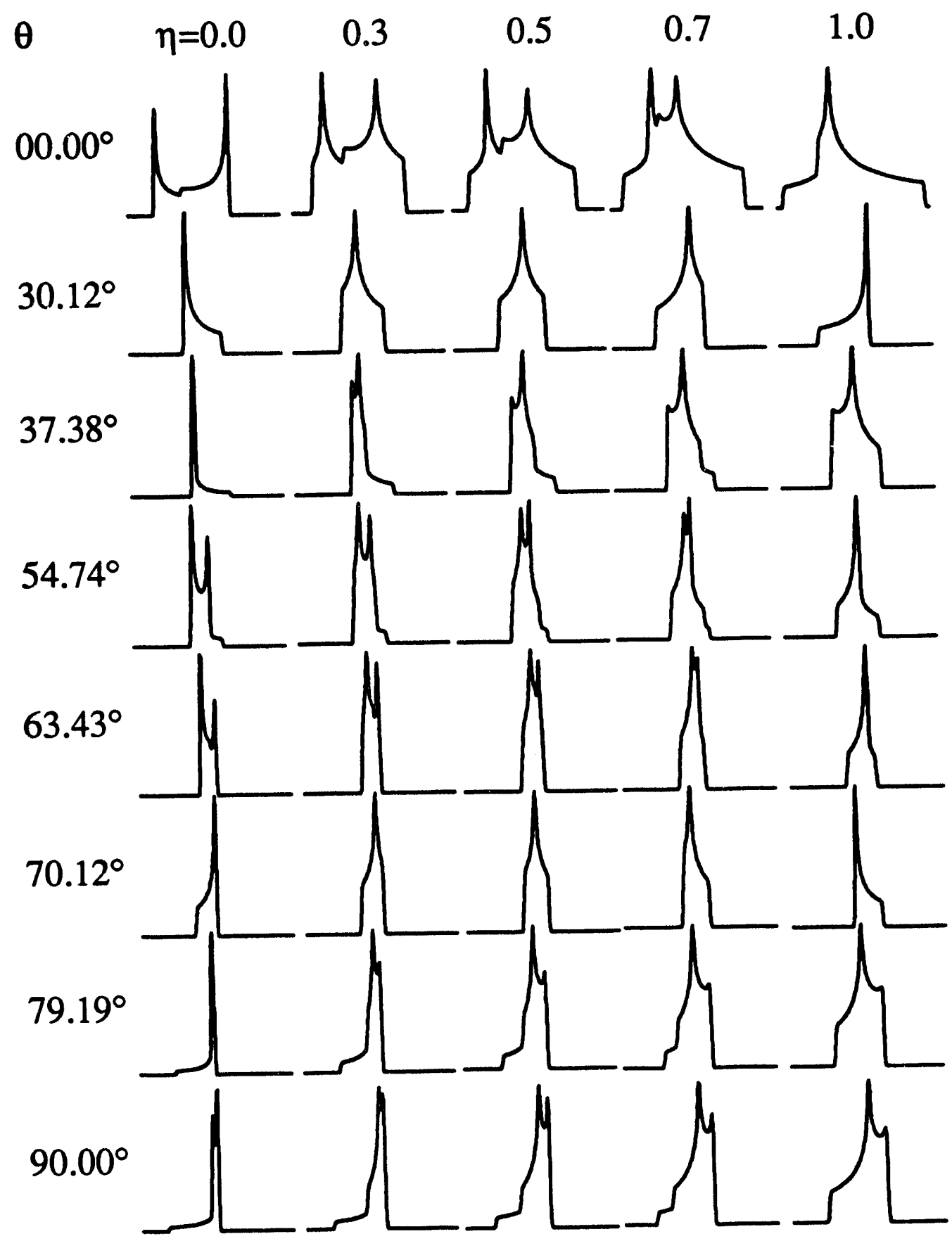

Figure 3.4: Variation of the powder lineshapes with different asymmetry parameters $\eta$ and different angles of the sample rotation axis with respect to the external field in VASS under fast spinning condition. $0^{\circ}$ (icosahedral angle), $30.56^{\circ}$ (root of $P_{4}\left(\cos \beta^{(4)}\right)$ ), $37.38^{\circ}$ (icosahedral angle), $54.74^{\circ}\left(\operatorname{root}\right.$ of $P_{2}\left(\cos \beta^{(2)}\right)$ ), $63.43^{\circ}$ (icosahedral angle), $70.12^{\circ}$ (root of $P_{4}\left(\cos \beta^{(4)}\right)$ ), $79.19^{\circ}$ (icosahedral angle), $90^{\circ}$ (icosahedral angle) 

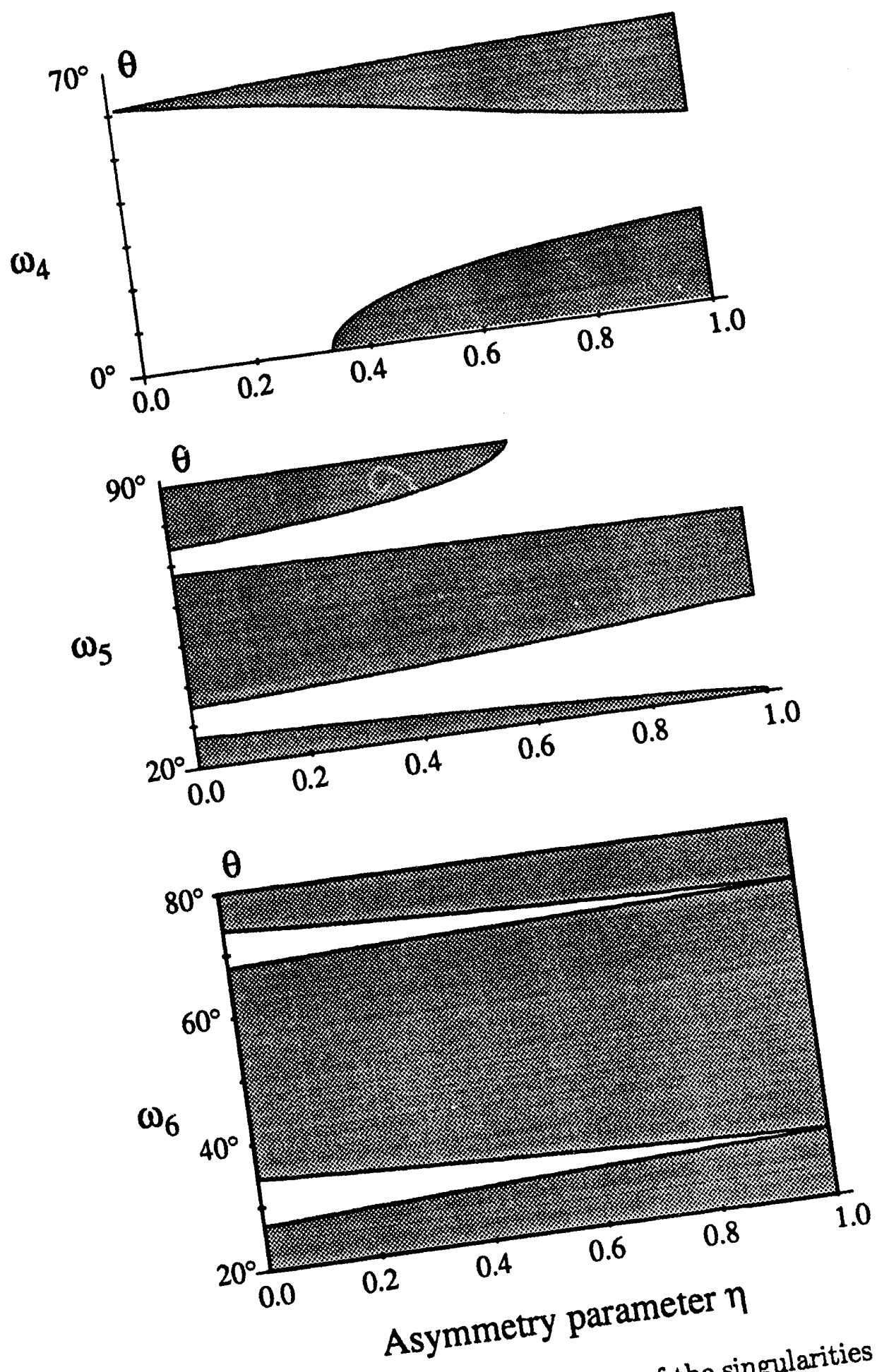

ing arities with the angle,

Figure 3.5: Variations of the boundary conditions of the sing shaded areas represent the existence of $\theta$, of the sample spinning axis in singularities for a particular ang $70.12^{\circ}$, of the fourth order the sample spins in any one of the magic angles, $30.56^{\circ}$ and $7 \omega_{4}, \omega_{5}$, and $\omega_{6}$. Whes the resulting powder lineshapes clearly seen in the singularity three singularities and anisotropy interactions (CSA). these two gaps, there are arising from chemical 83 

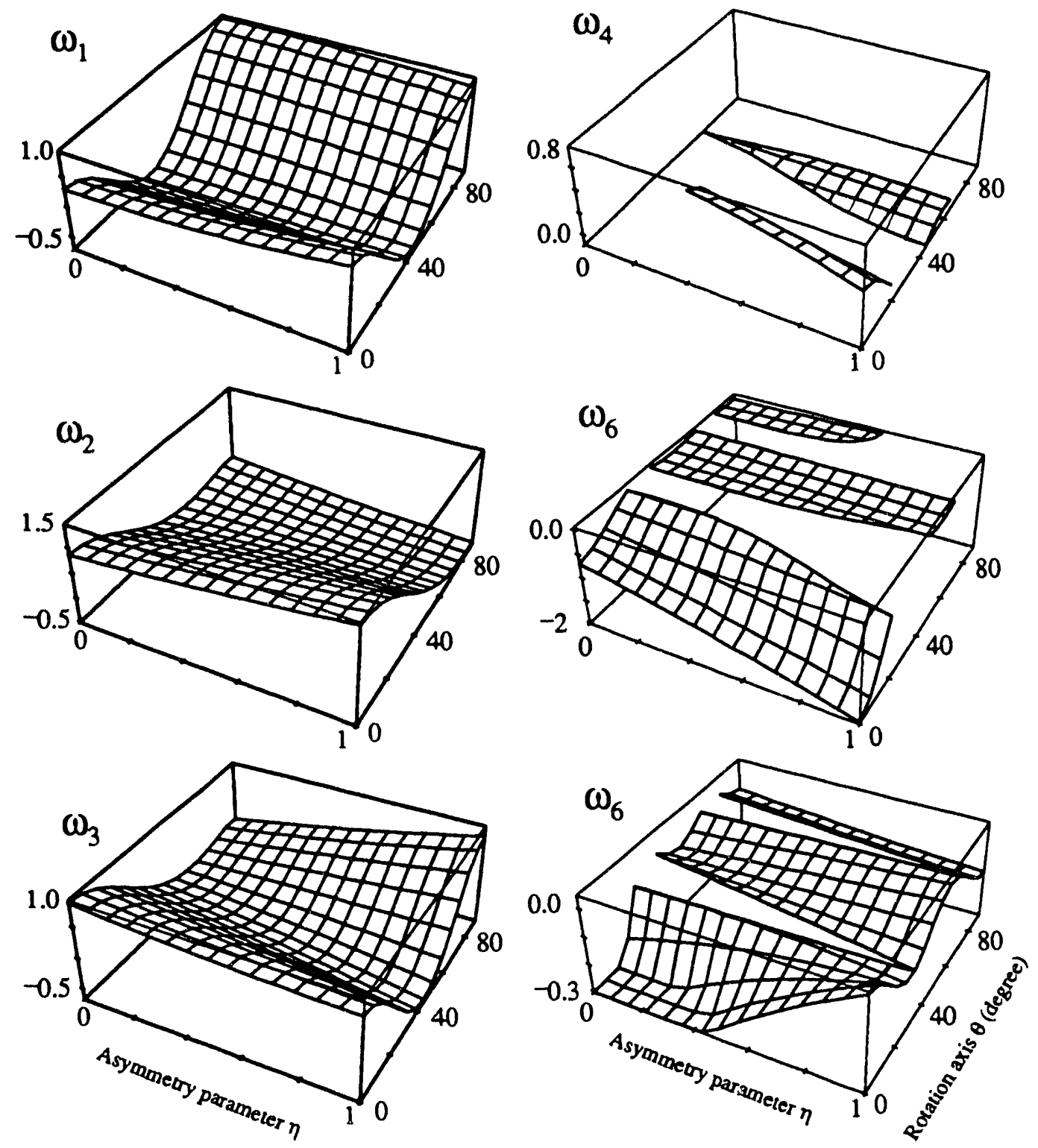

Figure 3.6: Three-dimensional graphs for the representation of the frequency variations of the singularities as angles of the rotation axis, $\theta$, and the asymmetry parameters, $\eta$, change. 

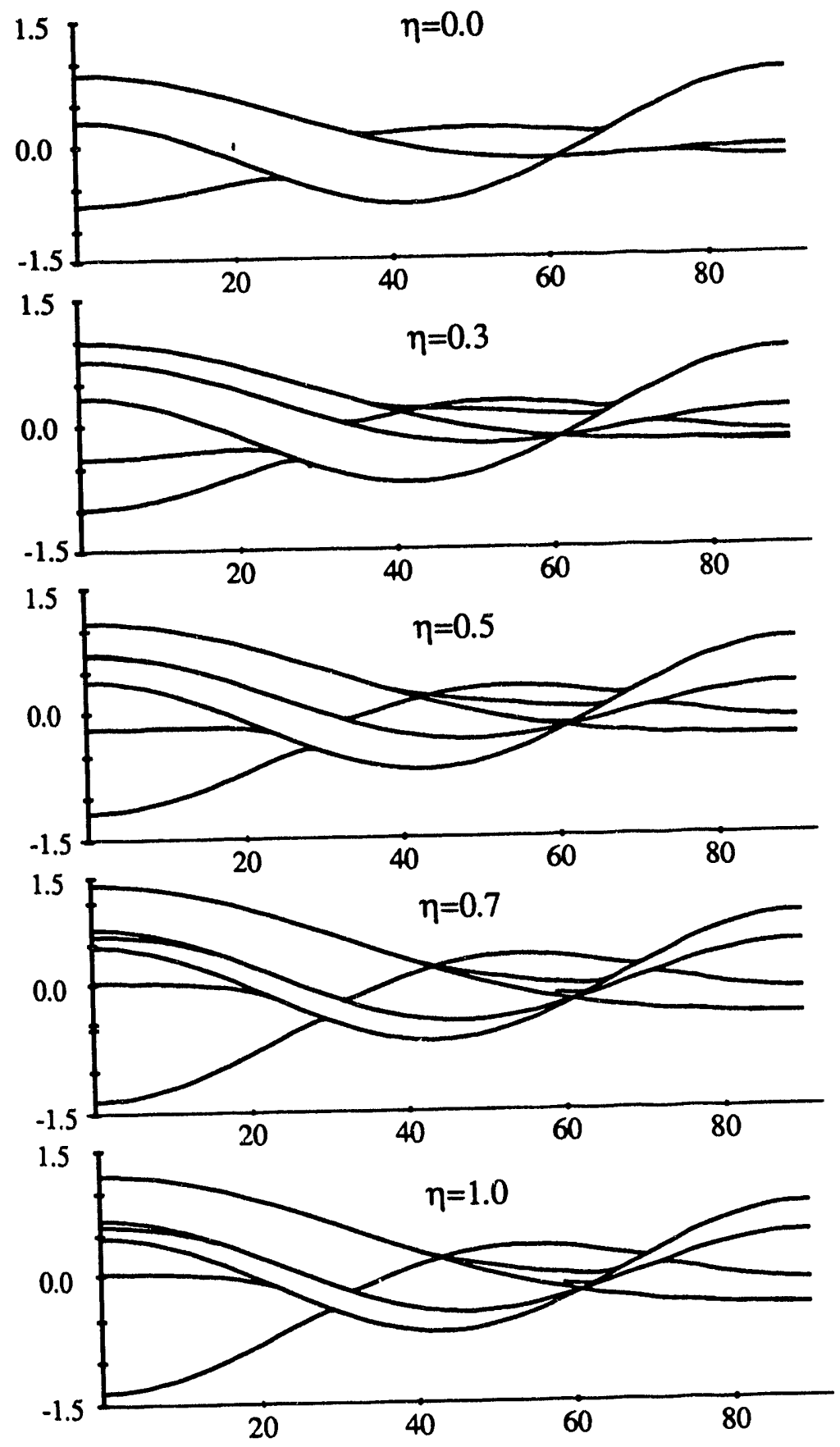

\section{Rotation axis Angles(degree)}

Figure 3.7: Variation of the frequencies of the singularities with different asymmetry parameters, $\eta$, as the angle of the rotation axis, $\theta$ increases from $0^{\circ}$ to $90^{\circ}$. From the top to the bottom, the asymmetry parameter is $0,0.3,0.5,0.7$, and 1.0 respectively. 
parameters and angles of the rotation axis. The surfaces of the first three singularities are very smooth, the other three exhibit some discontinuous regions around the magic angle of the fourth order Legendre polynomial. For the fourth singularity, this region is very small. Figure (3.7) presents two-dimensional schemes of the frequency variations of the singularities with different asymmetry parameters as a function of the sample spinning axis angles. From the flanks of the figure it can be clearly seen that the most narrow widths of the powder lineshapes under VASS should appear between $60^{\circ}$ and $70^{\circ}$.

\subsection{Dynamic Angle Spinning (DAS)}

Using the lineshape analysis methods to obtain the principal values of quadrupolar interactions will not in general work in the case of many sites in the molecule being studied. This is because of the overlap among the lineshapes arising from different sites possessing different quadrupolar interactions in the molecule. In order to extract useful information (for instance the isotropic chemical shifts and the second order isotropic shifts arising from quadrupolar interactions), it is necessary to have a high resolution spectra consisting of isotropic shifts by eliminating the anisotropic broadenings owing to second order effects of the quadrupolar interactions in the central transitions with half integer spin nuclei. The first order inhomogeneous broadening which originates from this spin Hamiltonian can be efficiently averaged to zero by using magic angle spinning (MAS)[56, 58]. However after applying MAS to a powder sample originating in second order quadrupolar interactions, the linewidth is only reduced by about a factor of three. Even under VASS, there is no solution to completely eliminate second order broadenings (see Figure 3.4). This is because second order lineshapes are determined by both the second and fourth rank tensors simultaneously. Under the fast spinning condition, the coefficients of the second and fourth rank tensors are second and fourth order Legendre polynomial, $P_{2}(\cos \theta)$ and 
$P_{4}(\cos \theta)$. In the interval of $\left[0^{\circ}, 90^{\circ}\right]$, the node of $P_{2}$ is $\theta^{(2)}=54.74^{\circ}$, and nodes of $P_{4}$ are $\theta_{1}^{(4)}=30.56^{\circ}$ and $\theta_{2}^{(4)}=70.12^{\circ}$. There is no common node for $P_{2}$ and $P_{4}$ (see Figure 3.8) and therefore the sample spinning with only a single fixed rotation axis inclined at any angle with respect to the external field cannot eliminate all the anisotropic broadenings in the second order lineshapes.

According to Eq.(3.20), the second order frequency shifts of the central transitions are inversely proportional to the Larmor frequency $\omega_{0}$. In other words, the second order broadenings of powder samples will decrease as increasing the external magnetic field. As an example, let's consider a quadrupolar nucleus with coupling constant $\omega_{Q}=100 \mathrm{kHz}$ which is a typical value for a spin $\frac{3}{2}$ nucleus. In order to reach one hertz resolution of the spectrum, the Larmor frequency, $\omega_{0}$, of the spin nucleus has to be in the order of $\omega_{Q}^{2}=10 \mathrm{GHz}$, corresponding to a magnetic field between $10^{3} \mathrm{~T}$ to $10^{4} \mathrm{~T}$ for various gyromagnetic ratios. It is obviously impossible to obtain such high magnetic field with the modern technology, and therefore to eliminate the second order broadening by increasing the external magnetic field is not realistic. However it is still useful (and sometimes necessary) to measure the lineshape at different magnetic fields in order to distinguish the chemical shifts and the quadrupolar interactions. The lineshapes of second order quadrupolar interactions as a function of magnetic field strength can be seen more clearly in Figure (3.9).

Figure (3.10) shows the variation of the second order shifts of the central transitions of each individual crystals as the angle $\theta$ of the sample spinning axis changes with respect to the external field. In Figure (3.10a), each line corresponds to a particular crystal orientation chosen randomly in SFC. As can be seen, the second order frequency shifts of each individual crystals oscillate with different frequencies and different initial phases as the sample spinning axis angle changes from $0^{\circ}$ to $90^{\circ}$. However, if each line in (3.10a) is divided by the value of its first point (corresponding to $\theta=0^{\circ}$ ), all lines dramatically refocus at two points: one is at $\theta=0^{\circ}$ and the other 
(a)

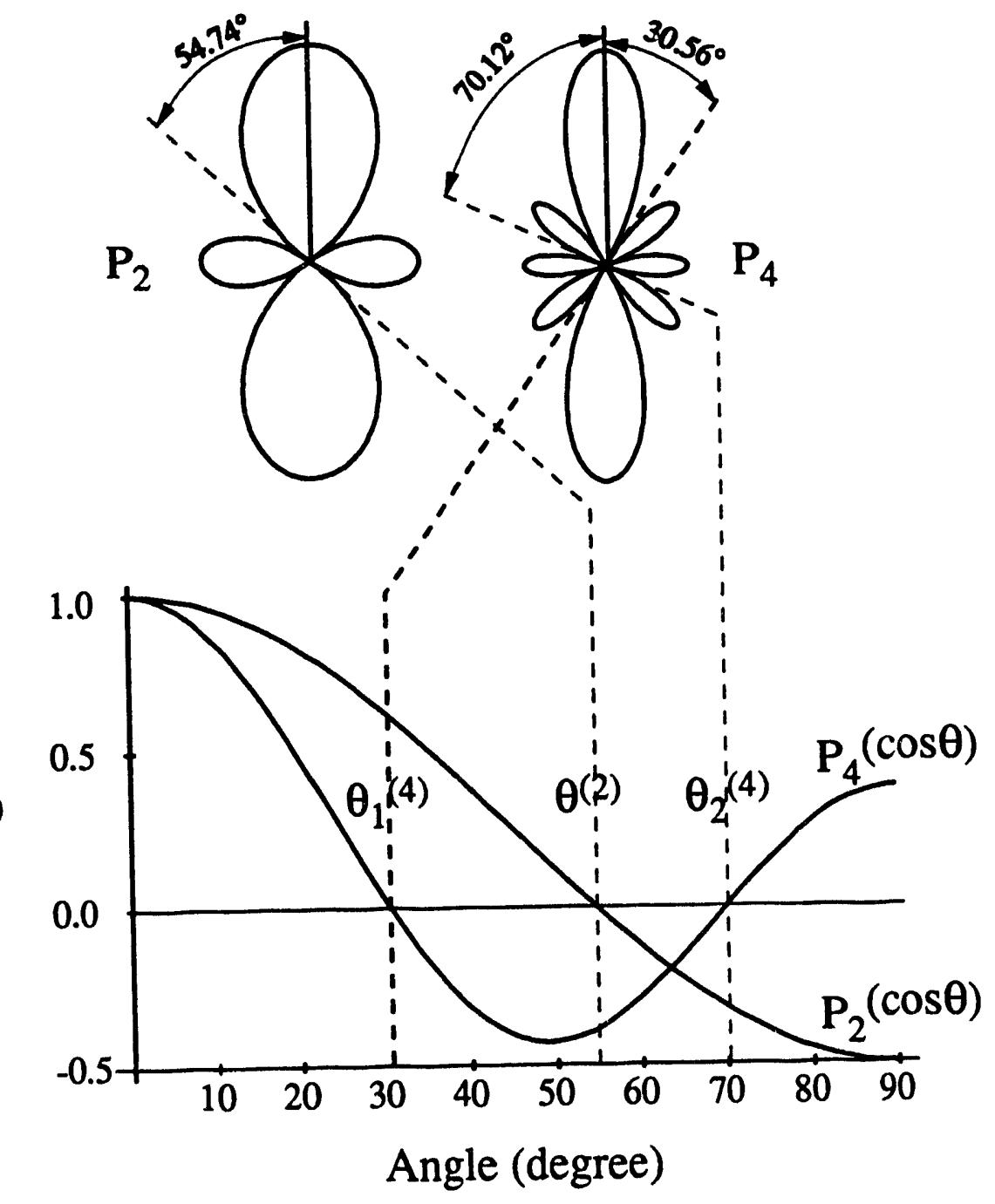

Figure 3.8: Plot of second and fourth order Legendre polynomial, $P_{2}(\cos \theta)$ and $P_{4}(\cos \theta)$ versus the angle of the rotation axis in VASS. (a) Plot in polar coordinates, (b) in Cartesian coordinates. The nodes of $P_{2}$ and $P_{4}$ are indicated by the dashed line. 


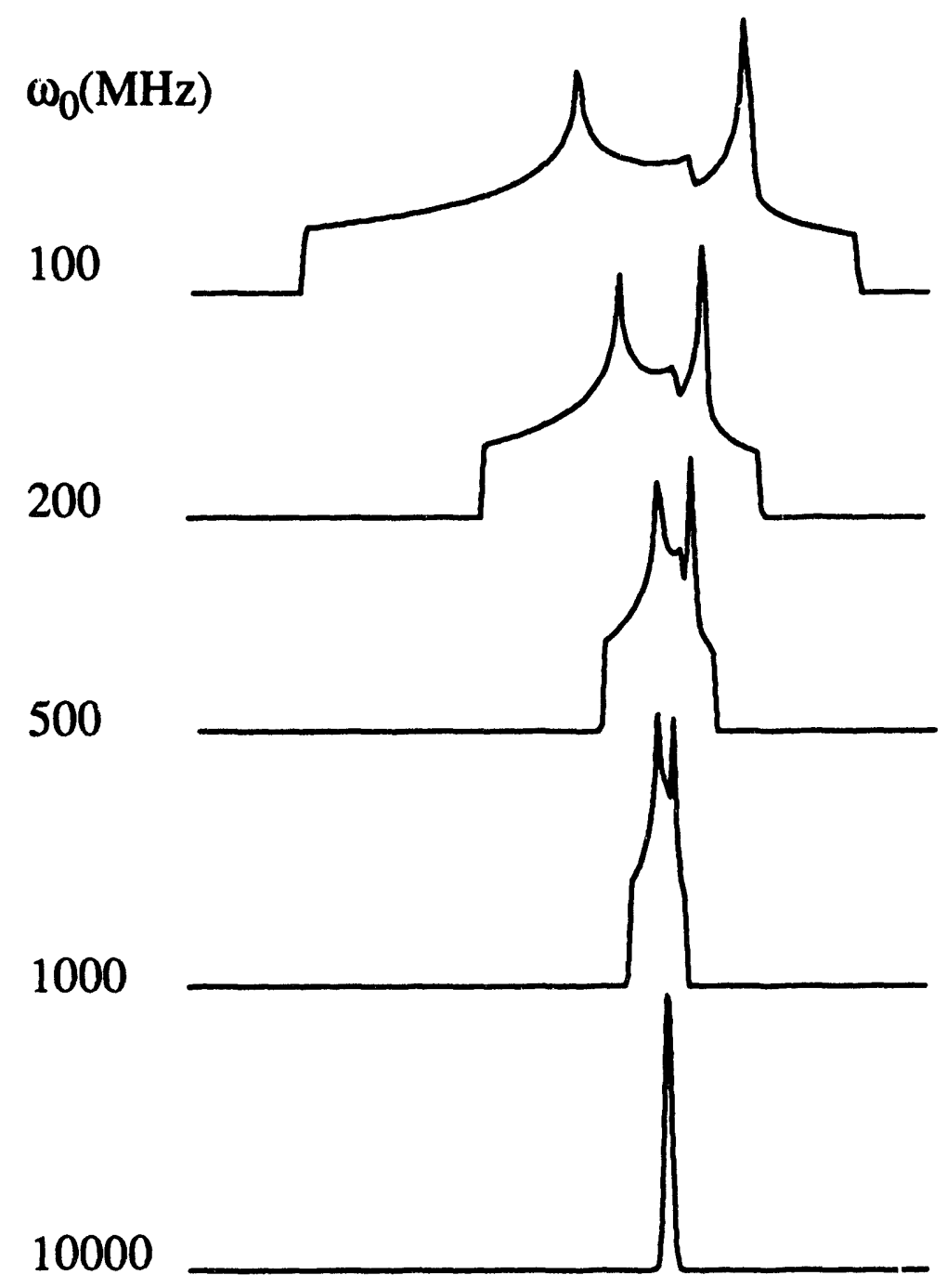

Figure 3.9: Variation of the second order powder lineshapes of the central transitions with a quadrupolar interaction as the magnetic field $H_{0}$ increases. The quadrupolar coupling constant, $\omega_{Q}$ is $100 \mathrm{KHz}$, and $\eta=0.5$. The spectrum will be high resolution once the Larmor frequency is in the order of $10 \mathrm{GHz}$. 
is at $\theta=63.43^{\circ}$. This feature tells us that although the second order shifts of the central transitions of differently oriented crystals in SFC vary differently with the sample spinning axis angle $\theta$, there are two positions, $\theta=0^{\circ}$ and $\theta=63.43^{\circ}$, of the sample spinning axis, at which the second order shifts of the central transitions are proportional each other. Even more interesting is that the proportional coefficient is a negative number. If a spin starts to process with a particular frequency determined by the orientation of the spin with respect to SFC at the first position (assume $\theta=0^{\circ}$ ), all spins will reverse the orientations of the procession after the sample spinning axis transfers from the first position to the second position $\left(\theta=63.43^{\circ}\right)$. The only difference is that the procession frequency are changed by a factor of five (see $(3.10 \mathrm{~b}))$. This scaling factor can be compensated by extending the evolution times of the spins at the second position five times. In other words, by properly setting up the experiment, the anisotropic broadening can be completely refocus by spinning the sample at two different axes during different evolution times. For the above example, the spins evolve for time $t_{1}$ during which the sample is rotating around the $Z_{L}$ axis, $\theta_{1}=0^{\circ}$, in the LAB frame and for time $t_{2}$ after the rotation axis is suddenly flipped to the angle of $\theta_{2}=63.43^{\circ}$ with respect to the external field. When $t_{2}=5 t_{1}$, all the anisotropic shifts will completely refocus, and an echo will appear. We call this echo the dynamic angle spinning (DAS) echo. In fact, such a behavior will happens if each line is divided by other positions of the sample spinning axis. (3.10c) shows another pair of angles of the sample spinning axis, $\theta_{1}=37.38^{\circ}$ and $\theta_{2}=79.19^{\circ}$, in which the scaling factor is one.

In order to apply the feature that the second order shifts of the central transitions change proportionally after the sample spinning axis transfers from one angle to another with respect to the external field, let us consider an ideal experiment in which the sample is spinning at an angle $\theta_{1}$ during evolution time $\Delta t_{1}$ and at another angle $\theta_{2}$ during $\Delta t_{2}$ achieved by suddenly flipping the rotation axis of the sample 
(a)

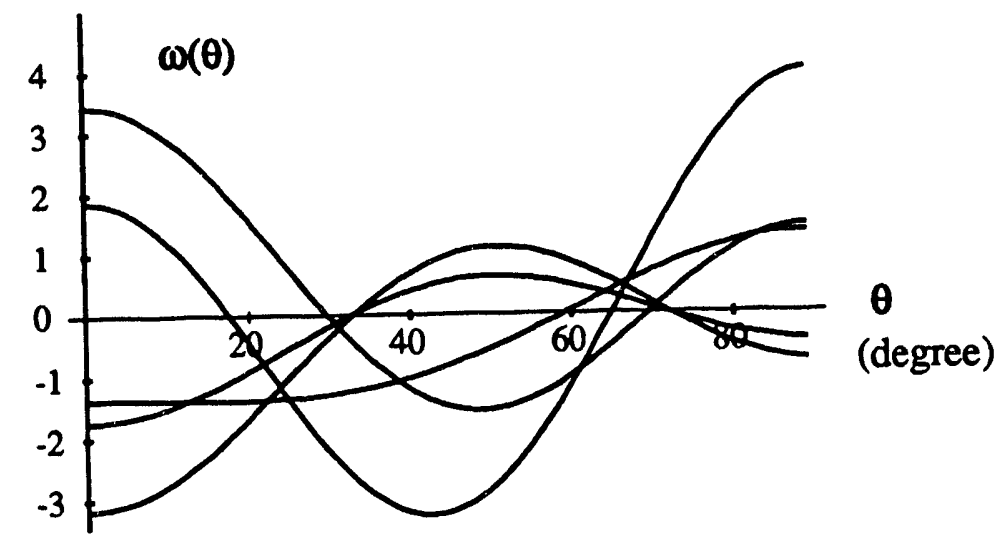

(b)

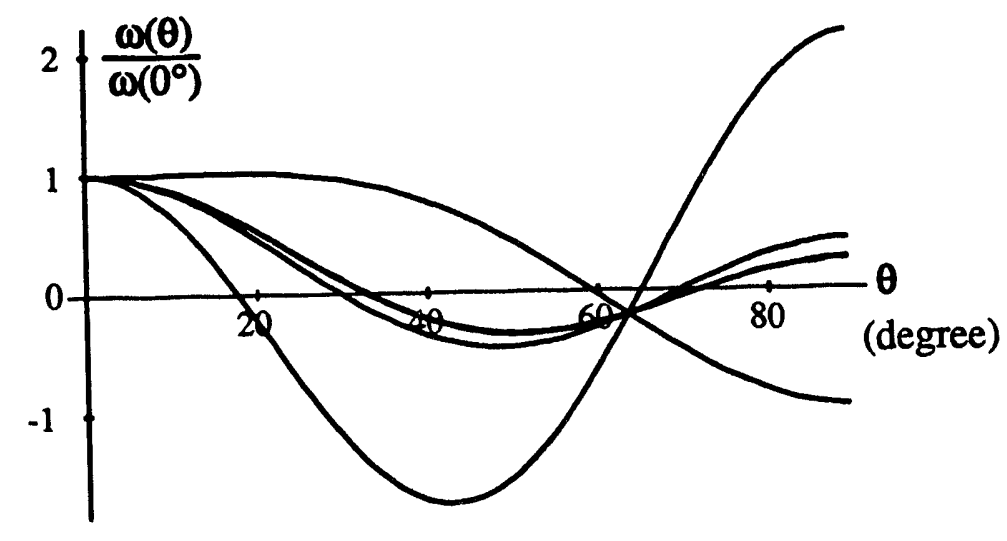

(c)

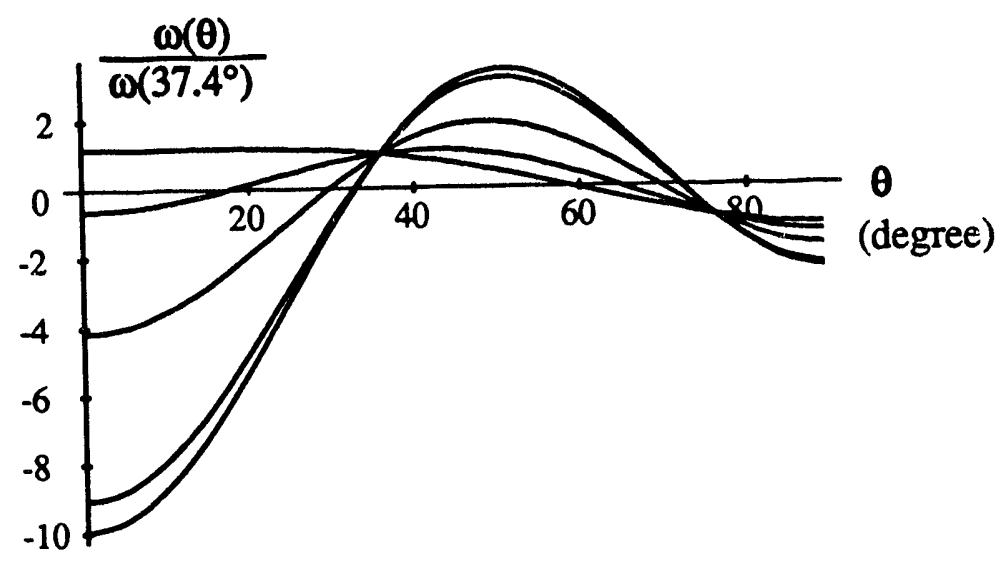

Figure 3.10: variation of the second order shifts with different angles of the sample spinning axis with respect to the external field. (a) Frequency dependence with the sample spinning axis angle, and each line corresponds to a particular crystal orientation chosen randomly in SFC. (b) After each line in (a) is divided by its first point corresponding to $\theta=0^{\circ}$, all shifts refocus at $\theta_{1}=0^{\circ}$ and $\theta_{2}=63.43^{\circ}$ and $\omega_{1}=-5 \omega_{2}$. (c), same as (b) but divided by the value at $\theta_{1}=37.38^{\circ}$, all lines then refocus at $\theta_{2}=79.19^{\circ}$ and $\omega_{1}=-\omega_{2}$ for all spins. 
between $\theta_{1}$ and $\theta_{2}$. This experiment is called dynamic angle spinning (DAS). For simplicity we assume that the sample rotation satisfies the fast spinning condition. The accumulated phases of the spin during evolution time $\Delta t_{1}$ and $\Delta t_{2}$ with spin Hamiltonian $\mathcal{H}_{1}$ and $\mathcal{H}_{2}$ are

$$
\begin{aligned}
& \varphi\left(\theta_{1}\right)=\sum_{l=2,4} C_{l} d_{0,0}^{(l)}\left(\theta_{1}\right) A_{l, 0} \Delta t_{1} \\
& \varphi\left(\theta_{2}\right)=\sum_{l=2,4} C_{l} d_{0,0}^{(l)}\left(\theta_{2}\right) A_{l, 0} \Delta t_{2},
\end{aligned}
$$

where the second order isotropic shift is not included because it is orientation independent. Since $\mathcal{H}_{1}$ and $\mathcal{H}_{2}$ commute with each other, the total phase at the end of the experiment is the sum of $\varphi\left(\theta_{1}\right)$ and $\varphi\left(\theta_{2}\right)$, that is

$$
\varphi\left(\theta_{1}, \theta_{2}\right)=\varphi\left(\theta_{1}\right)+\varphi\left(\theta_{2}\right)=\sum_{l=2,4} C_{l}\left[d_{0,0}^{(l)}\left(\theta_{1}\right) \Delta t_{1}+d_{0,0}^{(l)}\left(\theta_{2}\right) \Delta t_{2}\right] A_{l, 0}
$$

If we choose the total phase to be zero, it is necessary to satisfy the condition

$$
\begin{aligned}
& P_{2}\left(\cos \theta_{1}\right) \Delta t_{1}+P_{2}\left(\cos \theta_{2}\right) \Delta t_{2}=0 \\
& P_{4}\left(\cos \theta_{1}\right) \Delta t_{1}+P_{4}\left(\cos \theta_{2}\right) \Delta t_{2}=0
\end{aligned}
$$

where we replace $d_{0,0}^{(l)}$ by Legendre polynomial $P_{l}$ since $d_{0,0}^{(l)}=P_{l}$. The two pairs of angles shown in Figure (3.10b) and (3.10c) exactly satisfied the simultaneous Eqs.(3.29). Other solutions are schematically shown in Figure (3.11). In (a), $\theta_{1}$ and $\theta_{2}$ are plotted as function of the time ratio $k=\frac{\Delta t_{1}}{\Delta t_{2}}$ separately; in (b), a parametric graph with the time ratio, $k$, shows that there two regions in which Eq.(3.29) has solutions: one is related to $k$ and the other to $1 / k$. The analytical solution corresponding to the curves in Figure (3.11) are

$$
\begin{aligned}
& \cos ^{2}\left(\theta_{1}\right)=\frac{1}{3}\left(1 \mp \frac{2}{\sqrt{5}} \frac{1}{\sqrt{k}}\right) \\
& \cos ^{2}\left(\theta_{2}\right)=\frac{1}{3}\left(1 \pm \frac{2}{\sqrt{5}} \sqrt{k}\right)
\end{aligned}
$$

Each pair of rotation axis angles given by Eqs.(3.30) are called $D A S$ complementary angles. 

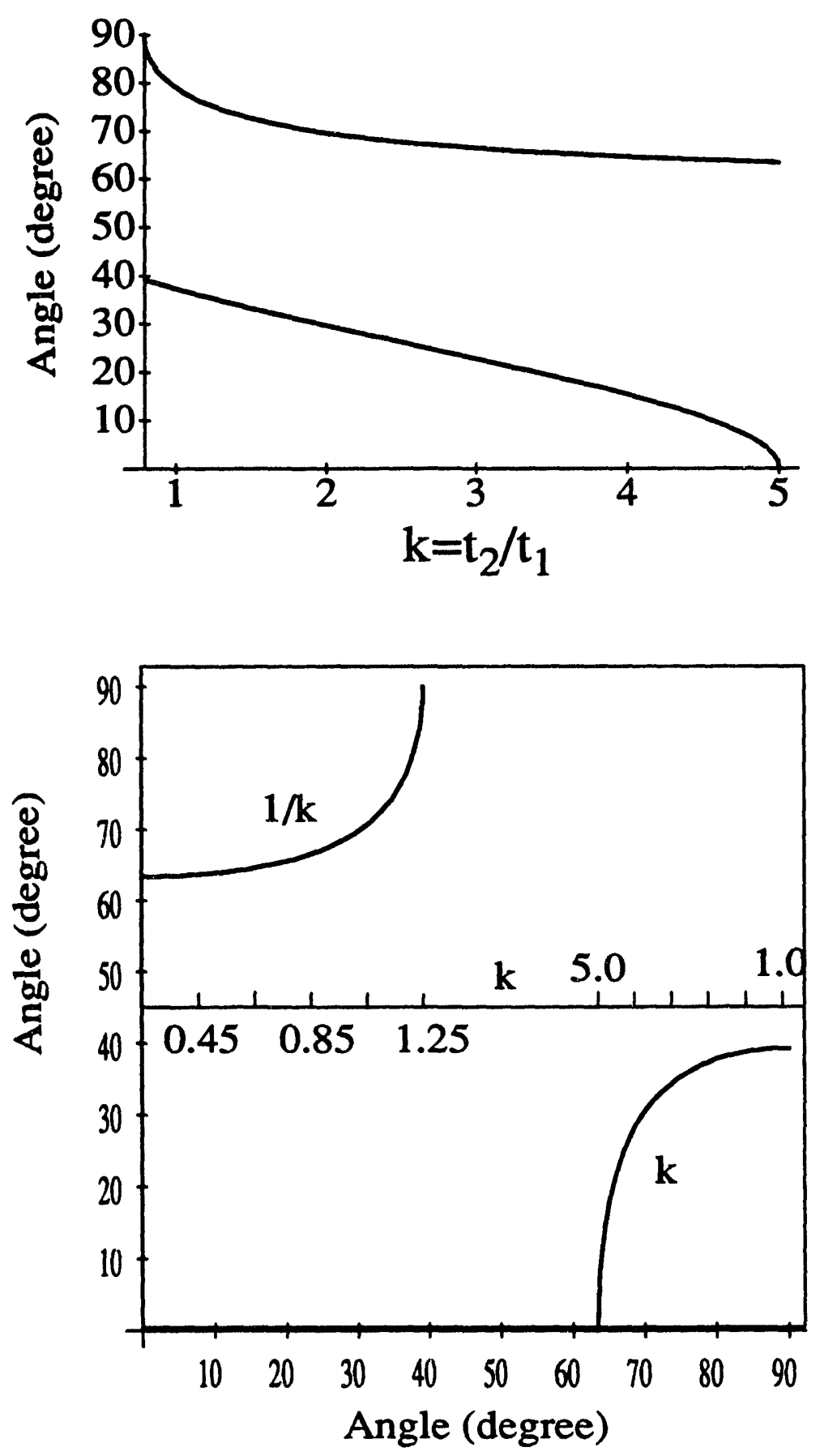

Figure 3.11: Graphic representation of DAS complementary angles resulting from of Eqs.(3.30) as a function of the time ratio $k=\frac{\Delta t_{1}}{\Delta t_{2}}$. (a) Individual plots of $\theta_{1}$ and $\theta_{2}$ versus $k$. (b) Parametric plot of the DAS angles with the time ratio $k$ shows the two sets of solutions of $\theta_{1}$ and $\theta_{2}$ : one is related to $k$ and the other to $\frac{1}{k}$, which are mirror image with each other about the diagonal line. 
Figure (3.12) shows simulated spectra for several pairs of DAS complementary angles with different asymmetry parameters. Each pair of the lineshapes in each box is mirror imaged about the isotropic shift. The first pair corresponds to the maximum scaling factor, $k=5$, which will lead the shortest time spun at $\theta_{1}$. The second pair is the solution of the magic angles of the fourth order Legendre polynomial $P_{4}(\cos \theta)$ where $k=1.87$. The third pair is choice actually used in the DAS experiment with $k=1$. One of the final pair is $\theta_{2}=90^{\circ}$, at which the maximum sensitivity of the signal can be received experimentally with $k=0.8$. The difference of the linewidths between two sets of spectra therefore is determined uniquely by the scaling factor $k=\frac{\Delta t_{1}}{\Delta t_{2}}$. For example, when $\Delta t_{1}=\Delta t_{2}, k$ is equal to unity and the solution is $\theta_{1}=37.38^{\circ}$ and $\theta_{2}=79.19^{\circ}$. This pair leads that the two sets of spectra has same linewidth.

It is well known that the MAS trajectory can be thought in a way that the magnetic field traverses on a cone in an octahedron in the SFC frame. The circle of the cone passes three vertices of the octahedron and the point of the cone is at the center of the octahedron (see Figure 3.13). Similarly the DAS trajectory with $k=1$ can also be thought in a way of that the magnetic field traverses on two cones whose circles pass the vertices of a dodecahedron and the points of the cones are the center of the dodecahedron in the SFC frame. Other solutions of the DAS trajectory also relate to a dodecahedron or an icosahedron. Later, we will show that the symmetry of the DAS trajectory is actually determined by the icosahedral group. For this reason, we list some of the angles in an icosahedron and a dodecahedron in Table (3.5).

The isotropic part in the spin Hamiltonian obviously will not be effected by the flippings of the rotation axis at the middle of the whole evolution. Experimentally, the data is acquired at the top of a DAS echo. The amplitude of the echo will therefore be modulated by the isotropic resonance frequencies of the spin nuclei. After Fourier 


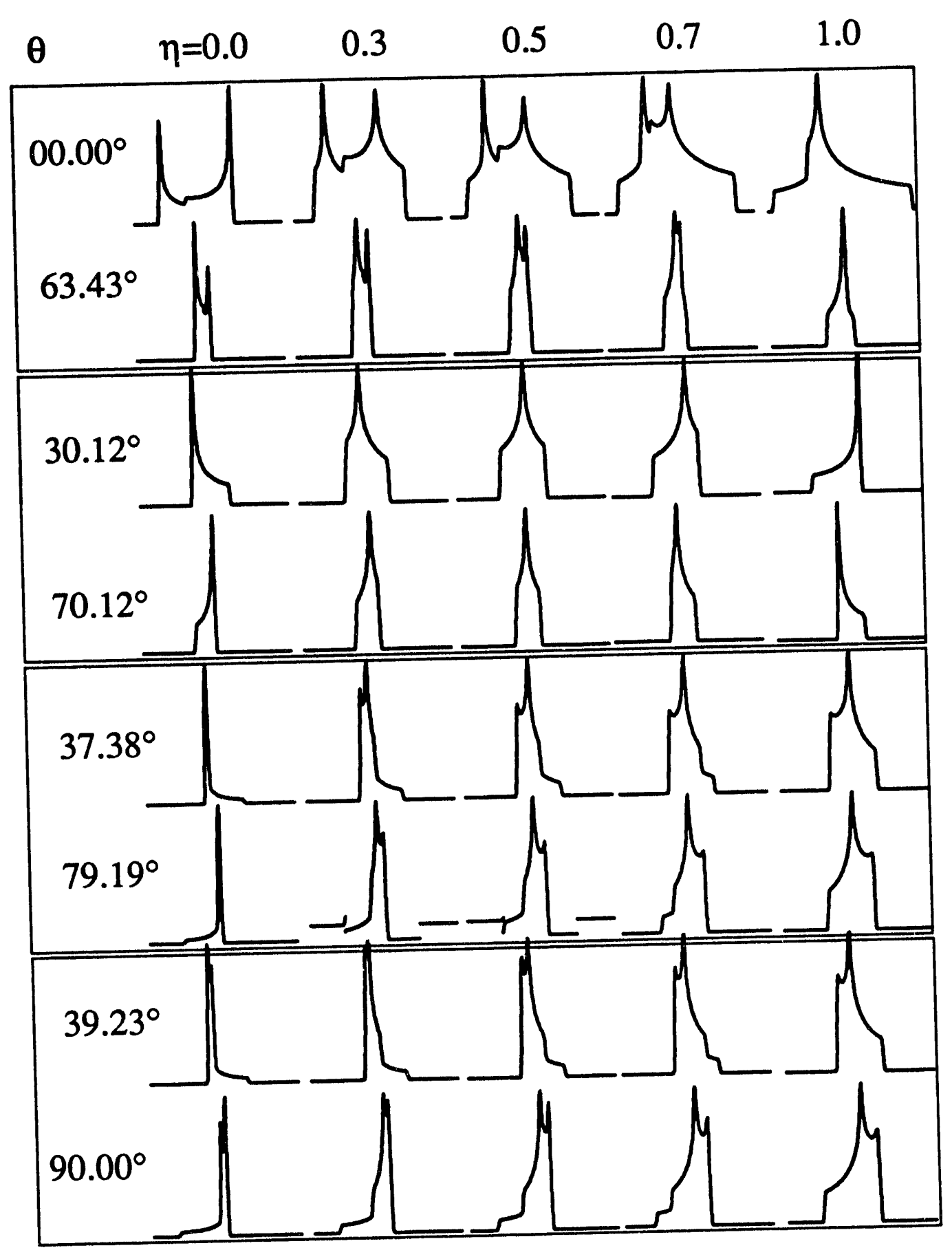

Figure 3.12: Calculated second-order powder patterns of the central transition with a spin $-\frac{3}{2}$ nuclei after motional averaging with a single rotation axis at various DAS complementary angles. The first pair, $0^{\circ}$ and $63.43^{\circ}$, is the icosahedral angles corresponding to the shortest time at $\theta_{1}$ and $k=5$. The second pair, $30.56^{\circ}$ and $70.12^{\circ}$, is the magic angles of the fourth order Legendre polynomial, $P_{4}(\cos \theta)$ with $k=1.87$, while the third pair is the dodecahedral angles and also the experimentally demonstrated $k=1$ case. The final pair, $39.23^{\circ}$ and $90^{\circ}$, has $k=0.8$, where the maximum sensitivity of the rf coil may be obtained. 

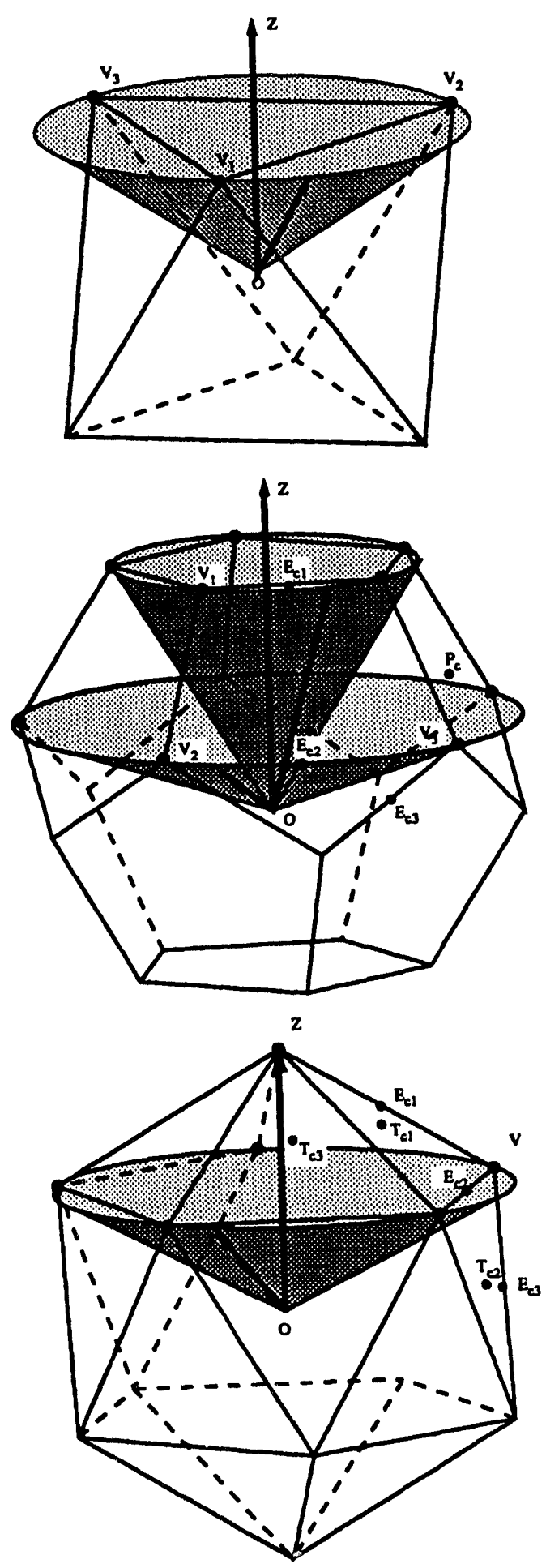

Figure 3.13: The external magnetic field, viewed in SFC, actually traverses on one or two cones in MAS and DAS. (top) The magic angle cone crosses three vertices of an octahedron in MAS. (middle) Two DAS cones cross ten vertices of a dodecahedron in $k=1$ case, while (bottom) is the DAS trajectory on an icosahedron. V is used to label a vertex, $\mathrm{P}$ for pentagon, $\mathrm{T}$ for triangle, and $\mathrm{E}$ for edge of the symmetry geometry, and $\mathrm{C}$ to label center and Latin letters in the indices to distinguish different centers 
Table 3.5: Angles in an icosahedron or a dodecahedron

\begin{tabular}{|c|c|c|c|c|c|c|}
\hline \hline Label & Dodecahedron & Icosahedron & Value & $\cos ^{2} \theta$ & $P_{2}(\theta)$ & $P_{4}(\theta)$ \\
\hline$\theta_{1}$ & $\overrightarrow{O Z O \vec{V}_{1}}$ & $\overrightarrow{O Z O \vec{T}_{c_{1}}}$ & $37.38^{\circ}$ & $\frac{\sqrt{5}+2}{3 \sqrt{5}}$ & $\frac{1}{\sqrt{5}}$ & $\frac{-\sqrt{5}}{9}$ \\
\hline$\theta_{2}$ & $\overrightarrow{O Z O \vec{V}_{2}}$ & $\overrightarrow{O Z O \vec{T}_{c_{2}}}$ & $79.19^{\circ}$ & $\frac{\sqrt{5}-2}{3 \sqrt{5}}$ & $\frac{-1}{\sqrt{5}}$ & $\frac{\sqrt{5}}{9}$ \\
\hline$\theta_{3}$ & $\overrightarrow{O Z O Z}$ & $\overrightarrow{O Z O Z}$ & $00.00^{\circ}$ & 1 & 1 & 1 \\
\hline$\theta_{4}$ & $\overrightarrow{O Z O \vec{P}_{c}}$ & $\overrightarrow{O Z O V}$ & $63.43^{\circ}$ & $\frac{1}{5}$ & $\frac{-1}{5}$ & $\frac{-1}{5}$ \\
\hline$\theta_{5}$ & $\overrightarrow{O Z O \vec{E}_{c_{1}}}$ & $\overrightarrow{O Z O \vec{E}_{c_{1}}}$ & $31.72^{\circ}$ & $\frac{\sqrt{5}+1}{2 \sqrt{5}}$ & $\frac{\sqrt{5}+3}{4 \sqrt{5}}$ & $-\frac{3-\sqrt{5}}{16}$ \\
\hline$\theta_{6}$ & $\overrightarrow{O Z O \vec{E}_{c 2}}$ & $\overrightarrow{O Z O \vec{E}_{c 2}}$ & $58.28^{\circ}$ & $\frac{\sqrt{5}-1}{2 \sqrt{5}}$ & $\frac{\sqrt{5}-3}{4 \sqrt{5}}$ & $-\frac{3+\sqrt{5}}{16}$ \\
\hline$\theta_{7}$ & $\overrightarrow{O Z O \vec{E}_{c 3}}$ & $\overrightarrow{O Z O \vec{E}_{c 3}}$ & $90.00^{\circ}$ & 0 & $-\frac{1}{2}$ & $\frac{3}{8}$ \\
\hline$\theta_{8}$ & $\overrightarrow{O \vec{V}_{1} O \vec{V}_{2}}$ & $\overrightarrow{O \vec{T}_{c_{1}} O \vec{T}_{c_{2}}}$ & $43.81^{\circ}$ & $\frac{5}{9}$ & $\frac{1}{3}$ & $-\frac{10}{27}$ \\
\hline$\theta_{9}$ & $\overrightarrow{O \vec{V}_{1} O \vec{V}_{3}}$ & $\overrightarrow{O \vec{T}_{c_{1}} O \vec{T}_{c_{3}}}$ & $70.53^{\circ}$ & $\frac{1}{9}$ & $-\frac{1}{3}$ & $\frac{1}{81}$ \\
\hline$\theta_{10}$ & $\overrightarrow{O \vec{V}_{2} O \vec{E}_{c_{1}}}$ & $\overrightarrow{O \vec{E}_{c_{1}} O \vec{T}_{c_{2}}}$ & $54.74^{\circ}$ & $\frac{1}{3}$ & 0 & $-\frac{7}{18}$ \\
\hline
\end{tabular}

where $O$ is the center, $V$ is the vertices of the dodecahedron and icosahedron, and $E_{c}$ is the centers of the edges, $T_{c}$ is centers of the triangles, and $P_{c}$ is the centers of the pentagons. Latin letters in the indices are used to distinguish different centers (see Figure 3.13).

transformation of the amplitude of the DAS echo, a high resolution spectrum can be obtained. The details about the DAS experiment[80] will be described in next section and the physical insight about DAS also will be theoretically thoroughly treated in the final section of this chapter.

\subsection{DAS Experiments}

Preliminary experiments were performed on Bruker AM-400 spectrometer. The magnetic field is $9.4 \mathrm{~T}$ and the resonance frequency for sodium-23 nuclei is $105.84 \mathrm{M} \mathrm{Hz}$. The whole DAS experimental setup is shown in Figure (3.14). It consists of a DAS probe, a step motor with the intelligent motor controller (IMC), and an IBM-PC computer [81]. In the DAS experiment, the flippingable probehead is driven by the 
step motor which is instructed by IMC. The functions of the step motors are programed by use of IBM-PC computer and their codes are then loaded into IMC. All instructions of IMC are triggered by TTL pulses from the AM- 400 spectrometer in order to synchronize with the pulse sequence. The motor is coupled to the flipping probehead via a Kevlar string. The reason we choose Kevlar string is that it has very small stretch under large force such that the vibration during flipping can be minimized in reaching the fastest flipping. The probehead is similar to the common cylindrical MAS probehead additionally suppled a puller which allows the probehead is flippingable. The coil is wrapped around the sleeve of the spinner in order to have maximum filling factor. The rf pulse is input via two pins which function as the flipping axis of the probehead. With controller feedback from an encoder attached to the motor, precise movement during the hop and reproducible values of initial and final positions for the experiment are accomplished.

In calibration of two angles $\sigma_{1}$ and $\theta_{2}$, we first set the magic angle $\theta=54.74^{\circ}$ by maximizing the rotational echoes in a bromine-81 FID from solid $\mathrm{KBr}$ (in some cases we also used deuterated hexamethylbenzene (HMB) to adjust the magic angle[83]), which was packed in the sample spinner along with a sodium-23 powder sample. The reason we choose $\mathrm{KBr}$ to assign the magic angle is that the resonance of the bromine81 nuclei is close to that of sodium-23, and the quadrupolar coupling constant of the bromine-81 nuclei is not extremely large so that the rotational echoes in an FID signal arising from the first order contribution of the quadrupolar interaction can be clearly seen once the sample is spinning at magic angle. After setting the spinning axis to the magic angle, the probehead is moved to $\theta_{1}$ by stepping the motor, and the number of steps can be calculated from the motor resolution of $0.36^{\circ}$ obtained using a thousand step incremental encoder and a hundred step motor with one sixteenth of a step capability, leading to comparable angular resolution in the probehead position due to a 1:1 coupling ratio between the step motor and the probehead. 


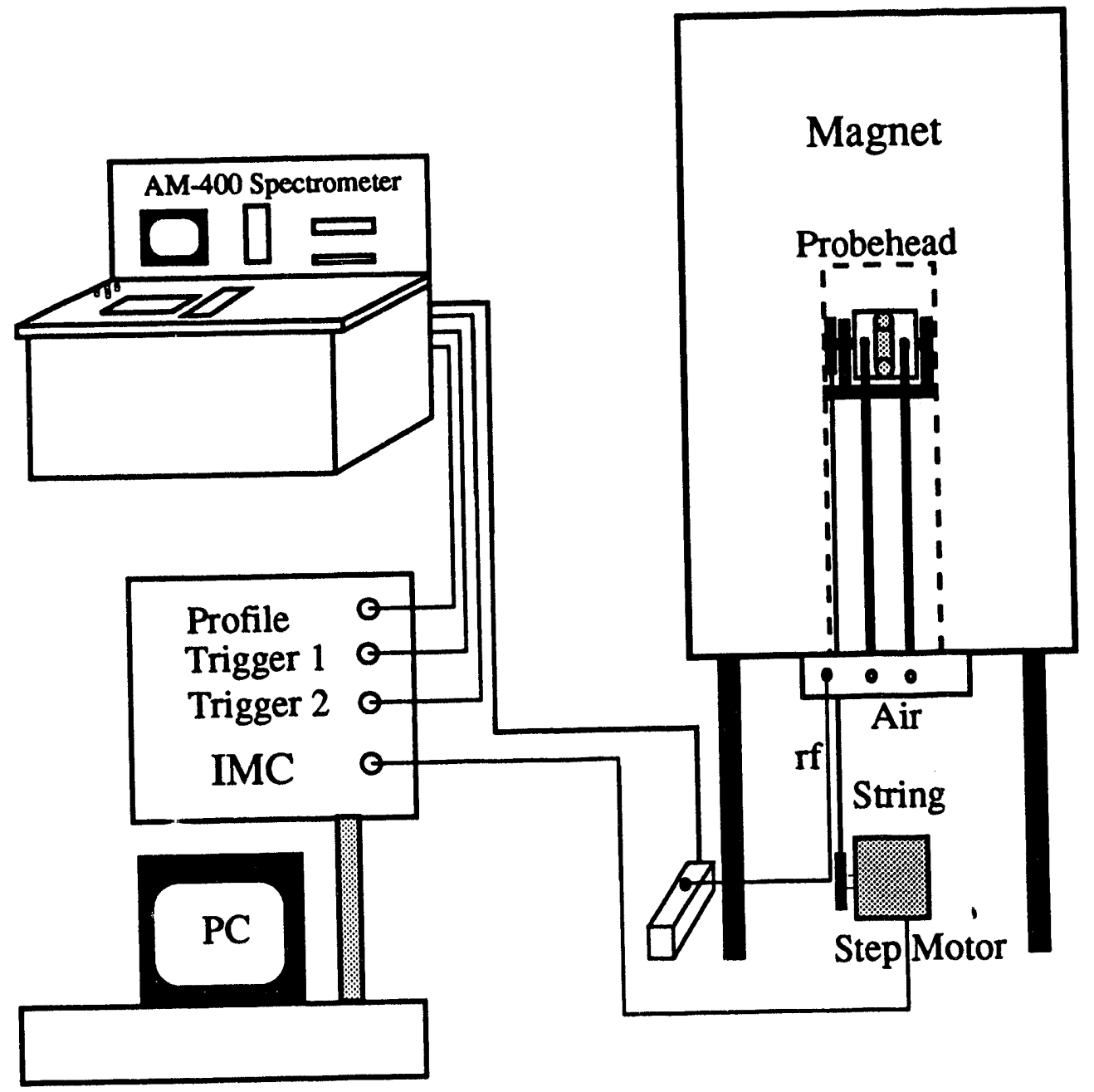

Figure 3.14: DAS experimental setup. The flippingable probehead is driven by a step motor which is fully programmable via a IBM-PC and the code then is loaded into the intelligent motor controller (IMC) and finally its function is triggered by TTL pulse from AM-400 to synchronize the pulse sequence. 
(a) If Pulses

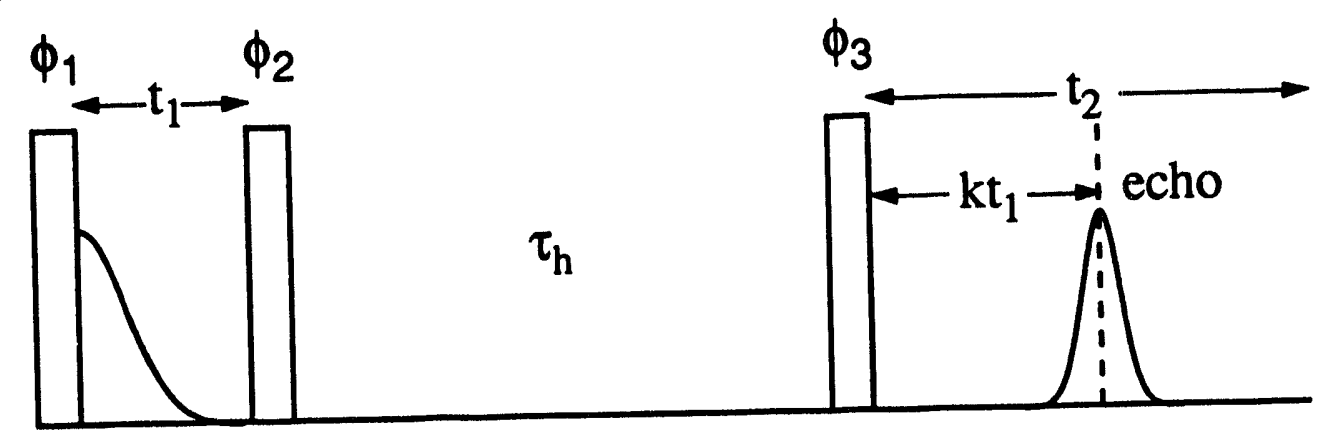

(b) rotor axis

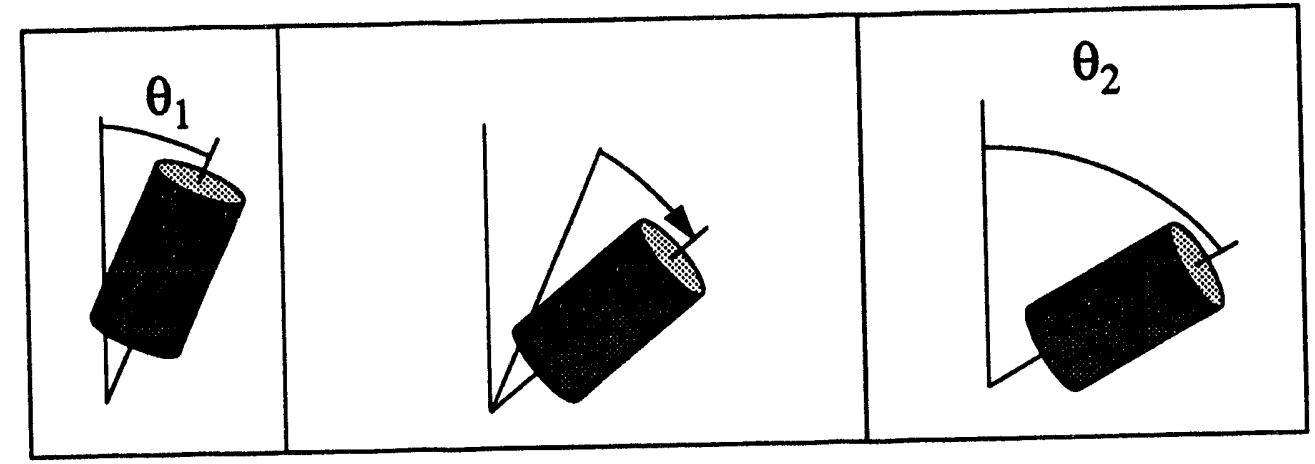

Figure 3.15: Pulse sequence applied in DAS experiment. All three pulse are $90^{\circ}$ for the central transition, and their phases are tabulated in Table (3.6). 
Figure (3.15) shows the pulse sequence and the spinner positions. Since the quadrupolar coupling constant is much larger than the strength of the rf field for half integer spin nuclei, the response of the magnetization for central transition to a rf pulse is same as a selective pulse to the pair of energy levels with $m= \pm \frac{1}{2}$ $[84,85,86]$. The length of a $90^{\circ}$ pulse is scaled by a factor of $2 /(2 I+1)$ in comparison with the one measured in liquid sample with same rf field. For sodium-23 nuclei with large quadrupolar interaction, the $90^{\circ}$ pulse length is $6.2 \mu \mathrm{sec}$ at $\theta_{1}=37.38^{\circ}$ and $4.1 \mu \mathrm{sec}$ at $\theta_{2}=79.19^{\circ}$ while that of $\mathrm{NaCl}$, whose EFG is zero due to high symmetry molecule structure, doubles the above values.

One important concept used in the DAS pulse sequence is the storage of a magnetization. It relates to the fact that the relaxation of a magnetization is inhomogeneous. For example in solids the transverse relaxation is usually much faster than the longitudinal relaxation. If the transverse component is transferred to the longitudinal component, it can therefore relax in much longer times than in the transverse plane. We called this the storage of the magnetization, first used in the magic angle hopping experiments $[87,88]$.

The magnetization of samples with large quadrupolar interactions and chemical shift anisotropies is brought into $x y$ plane by applying a $90^{\circ}$ pulse along the $x$-axis in the rotating frame, where it evolves for a length of time $t_{1}$. The density matrix at the end of the evolution time $t_{1}$ is

$$
\rho\left(t_{1}\right)=-\cos \left(\omega_{Q}^{(2)}\left(\theta_{1}\right) t_{1}\right) \mathbf{I}_{y}^{\frac{1}{2}-\frac{1}{2}}+\sin \left(\omega_{Q}^{(2)}\left(\theta_{1}\right) t_{1}\right) \mathbf{I}_{x}^{\frac{1}{2}-\frac{1}{2}}
$$

where

$$
\omega_{Q}^{2}(\theta)=\sum_{l} C_{l} d_{0,0}^{(l)}(\theta) A_{l, 0}
$$

and $\mathrm{I}_{x}^{\frac{1}{2}-\frac{1}{2}}$ and $\mathrm{I}_{y}^{\frac{1}{2}} \frac{-1}{2}$ are the fictitious operators for central transitions $[84,85,86]$. The second pulse along $x$ axis rotates $I_{y}^{\frac{1}{2}-\frac{1}{2}}$ component of the density matrix to along the external magnetic field where the evolved density matrix is stored during flipping the 
sample spinning axis from $\theta_{1}$ to $\theta_{2}$. After this flipping, the stored magnetization is rotated back into the $x y$ plane by the third pulse along the $x$ axis. After the density matrix evolves for a length of time $t_{2}$, it becomes

$$
\begin{aligned}
\rho\left(t_{1}, t_{2}\right) & =\frac{1}{2}\left[\cos \left(\omega_{Q}^{(2)}\left(\theta_{1}\right) t_{1}+\omega_{Q}^{(2)}\left(\theta_{2}\right) t_{2}\right)+\cos \left(\omega_{Q}^{(2)}\left(\theta_{1}\right) t_{1}-\omega_{Q}^{(2)}\left(\theta_{2}\right) t_{2}\right)\right] \mathbf{I}_{y}^{\frac{1}{2}-\frac{1}{2}} \\
& \left.+\frac{1}{2}\left[\sin \left(\omega_{Q}^{(2)}\left(\theta_{1}\right) t_{1}+\omega_{Q}^{(2)}\left(\theta_{2}\right) t_{2}\right)-\sin \left(\omega_{Q}^{(2)}\left(\theta_{1}\right) t_{1}-\omega_{Q}^{(2)}\left(\theta_{2}\right) t_{2}\right)\right]\right]_{x}^{\frac{1}{2}-\frac{1}{2}}
\end{aligned}
$$

According to Eq.(3.29), when $t_{2}=k t_{1}$, i. DAS echo forms with two residual components: the relaxed magnetization during flipping which are ignored in the present derivation, and the double precession magnetization. These residual components can be eliminate experimentally by use of the phase cycling listed in Table (3.6).

\begin{tabular}{|c|c|c|c|c|c|c|}
\hline$\phi_{1}$ & $\phi_{2}$ & $\phi_{3}$ & $\phi_{r}$ & $\phi_{i}$ & Real & Imaginary \\
\hline$X$ & $X$ & $X$ & $Y$ & $\bar{X}$ & $C_{+}+C_{-}$ & $S_{+}-S_{-}$ \\
\hline$X$ & $\bar{X}$ & $\bar{X}$ & $Y$ & $\bar{X}$ & $C_{+}+C_{-}$ & $S_{+}-S_{-}$ \\
\hline $\bar{X}$ & $X$ & $X$ & $\bar{Y}$ & $X$ & $C_{+}+C_{-}$ & $S_{+}-S_{-}$ \\
\hline $\bar{X}$ & $\bar{X}$ & $\bar{X}$ & $\bar{Y}$ & $X$ & $C_{+}+C_{-}$ & $S_{+}-S_{-}$ \\
\hline$Y$ & $X$ & $X$ & $\bar{X}$ & $Y$ & $C_{+}-C_{-}$ & $S_{+}+S_{-}$ \\
\hline$Y$ & $\bar{X}$ & $\bar{X}$ & $\bar{X}$ & $Y$ & $C_{+}-C_{-}$ & $S_{+}+S_{-}$ \\
\hline $\bar{Y}$ & $X$ & $X$ & $X$ & $\bar{Y}$ & $C_{+}-C_{-}$ & $S_{+}+S_{-}$ \\
\hline $\bar{Y}$ & $\bar{X}$ & $\bar{X}$ & $X$ & $\bar{Y}$ & $C_{+}-C_{-}$ & $S_{+}+\vdots_{-}$ \\
\hline \multicolumn{5}{|c|}{ sum } & $8 C_{+}$ & $8 S_{+}$ \\
\hline
\end{tabular}

Table 3.6: Phase cycling in DAS experiment

in this table $\phi_{1}, \phi_{2}$ and $\phi_{3}$ are the phases of the first, second and the third pulse respectively, and $\phi_{r}\left(\phi_{i}\right)$ is the real (imaginary) buffer detection phase. $C_{ \pm}=\frac{1}{2} \cos \left(\omega_{Q}^{(2)}\left(\theta_{1}\right) t_{1} \pm \omega_{Q}^{(2)}\left(\theta_{2}\right) t_{2}\right)$ and $S_{ \pm}=\frac{1}{2} \sin \left(\omega_{Q}^{(2)}\left(\theta_{1}\right) t_{1} \pm \omega_{Q}^{(2)}\left(\theta_{2}\right) t_{2}\right)$.

Consider the experiment illustrated in Figure (3.15) with the phases of the pulses assigned in Table (3.6) with a sample of polycrystalline sodium sulfate $\left(\mathrm{Na}_{2} \mathrm{SO}_{4}\right)$ 
using an axis flip from $\theta_{1}=37.38^{\circ}$ to $\theta_{2}=79.19^{\circ}$ and the evolution times $t_{1}=$ $t_{2}=1.5 \mathrm{msec}$. The quadrupolar coupling constant of sodium nuclei in the sample is $e^{2} Q q / h=2.6 \mathrm{MHz}$ and the asymmetry parameter is $\eta=0.6$. After the first pulse, while the sample is spinning at $\theta_{1}$ with respect to the external field, the magnetization evolves at the frequencies $\omega_{Q}^{(2)}\left(\theta_{1}\right)$. An oscilloscope trace of the decay of the signal is shown in Figure (3.16a). After 1.5msec, a second pulse is applied to store a component of the magnetization and then the spinner is performed a flip to $\theta_{2}$. Following the third pulse which brings the evolved magnetization into the $x y$ plane again, the evolution frequencies of the each individual spins are $\omega_{Q}^{(2)}\left(\theta_{2}\right)$, opposite to the previous precessing frequencies. All the magnetization therefore refocuses after $1.5 \mathrm{msec}$ and a full echo may be reconstructed after eight experiments, as shown in Figure (3.16b). At the center of the echo, only the evolution of the isotropic component remains (in this experiment, isotropic shift had been set to zero). The echo clearly indicates the refocusing of the chemical shift anisotropy and of the second order quadrupolar broadenings. Other broadenings with bilinear spin operators in the spin interaction Hamiltonian (e.g. the dipolar interaction) cannot be effectively refocused because the other orthogonal components in the density matrix represented by the bilinear spin operators cannot be efficiently stored. The amplitudes of the echoes are therefore smaller than the initial value. Another reason why the echo amplitudes decrease is due to the effects of relaxation and spin diffusion happened during the storage time. Nevertheless, DAS is applicable to dilute spin nuclei, such as ${ }^{17} \mathrm{O}$.

The DAS experiment with $k=1$ is performed by increasing $t_{1}$ in steps of $\Delta t$, in the order of 10 to 100 microseconds. The effective bandwidth in the high resolution spectrum is $1 / 2 \Delta t$. Sodium-23 results have been obtained on both $\mathrm{Na}_{2} \mathrm{SO}_{4}$ and $\mathrm{Na}_{2} \mathrm{C}_{2} \mathrm{O}_{4}$, and they are presented in Figures (3.17) and (3.18). In Figure (3.17), the DAS echo amplitudes of sodium sulfate $\left(\mathrm{Na}_{2} \mathrm{SO}_{4}\right)$ were acquired in quadrature 

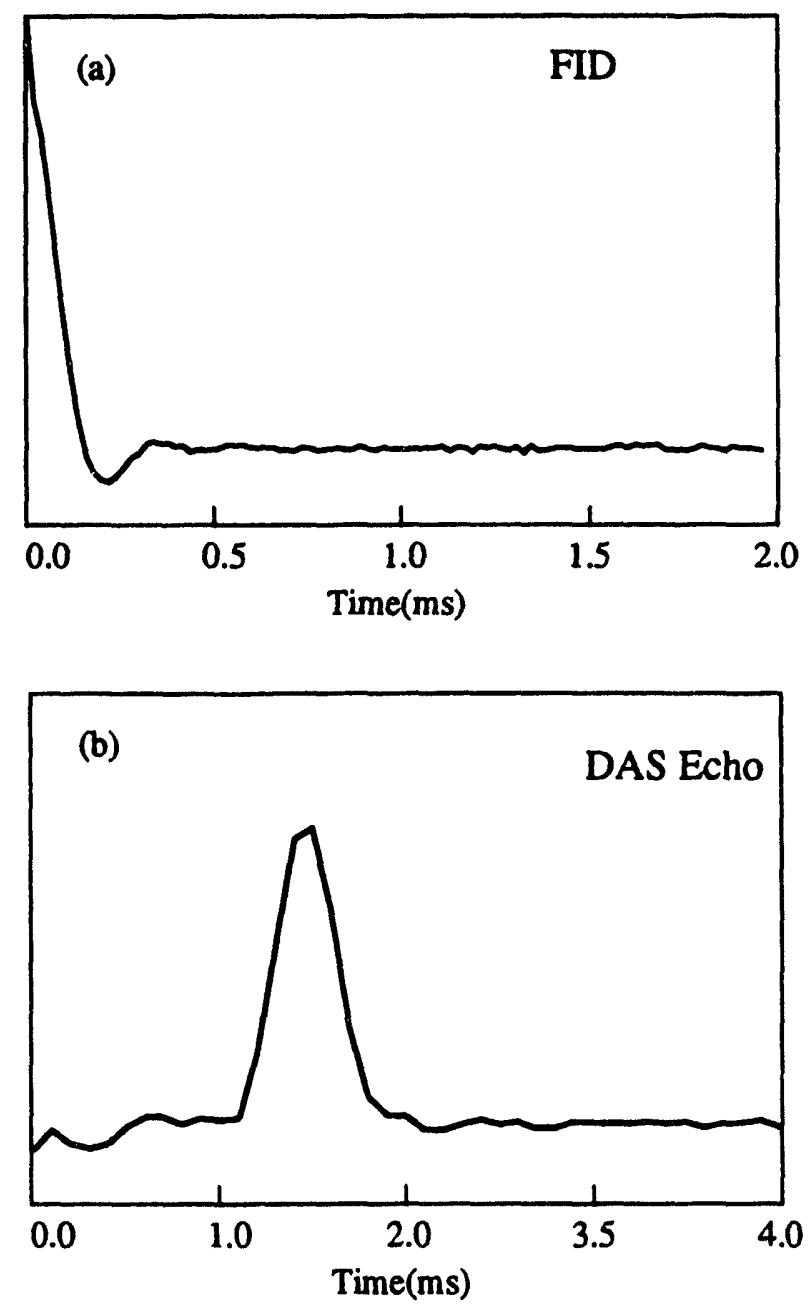

Figure 3.16: Demonstration of forming a rotational echo in a DAS experiment of polycrystalline sodium sulfate $\left(\mathrm{Na}_{2} \mathrm{SO}_{4}\right)$. (a) The FID signal of the central transitions after applying a $90^{\circ}$ pulse decays in time of $500 \mu \mathrm{sec}$. (b) An echo of the transverse magnetization occurs at $t_{1}=1.5 \mathrm{msec}$ after the third pulse in the DAS experiment. Experimental parameters is same as in (a). 
and the resulting FID in $t_{1}$ was Fourier transformed to produce the one-dimensional spectrum of Figure (3.17c). This high resolution spectrum contains one isotropic peak, shown at $0 \mathrm{~Hz}$ in the frequency domain, with a linewidth of $575 \mathrm{~Hz}$. The sideband pattern has a spacing of $1.64 \mathrm{kHz}$, which is one-half of the spinning speed of the sample. The appearance of the sidebands at half of the spinning speed can be expected since the evolution is divided into two periods with a storage of the magnetization between them (see next chapter for details). The linewidth of the central peak is narrowed by a factor of about seven in comparison with the MAS results in Figure (3.17b), and the isotropic frequency can easily be identified from the maximum of the symmetric central peak.

A two-dimensional DAS power spectrum for sodium-23 in polycrystalline $\mathrm{Na}_{2} \mathrm{C}_{2} \mathrm{O}_{4}$ is presented in Figure (3.18). The quadrupolar coupling constant of sodium nuclei is $\omega_{Q}=403 \mathrm{kHz}$ and the asymmetry parameter is $\eta=0.72$. Data were collected as a series of complex FIDs in two dimensions. The $t_{1}$ domain shows the evolution of the magnetization under spinning at the angle of $\theta_{1}$ which modulates the FID in the $t_{2}$ domain acquired when the sample is spinning at the angle $\theta_{2}$. After a twodimensional Fourier transformation is performed on the FID signals, a correlation spectrum between spinning at $\theta_{1}$ and $\theta_{2}$ may be obtained. The projection on the $F_{1}$ domain shows the powder lineshape spinning at the $\theta_{1}$ while the projection on the $F_{2}$ domain produces the lineshape spinning at the $\theta_{2}$. From the figure, it can be seen that the projection along the diagonal axis yields the high resolution DAS spectrum (Figure (3.17c)).

The residual linewidth is determined by the dipolar coupling between the spins. To prove this we have performed a Hahn echo experiment with $\mathrm{Na}_{2} \mathrm{SO}_{4}$ when the sample spins around the axis tilted at $\theta_{2}$; the decay of this echo is determined by the dipolar coupling between sodium nuclei scaled down by a factor of $P_{2}\left(\cos \theta_{2}\right)$. It has been pointed out above that the DAS experiment only stores two linear orthog- 


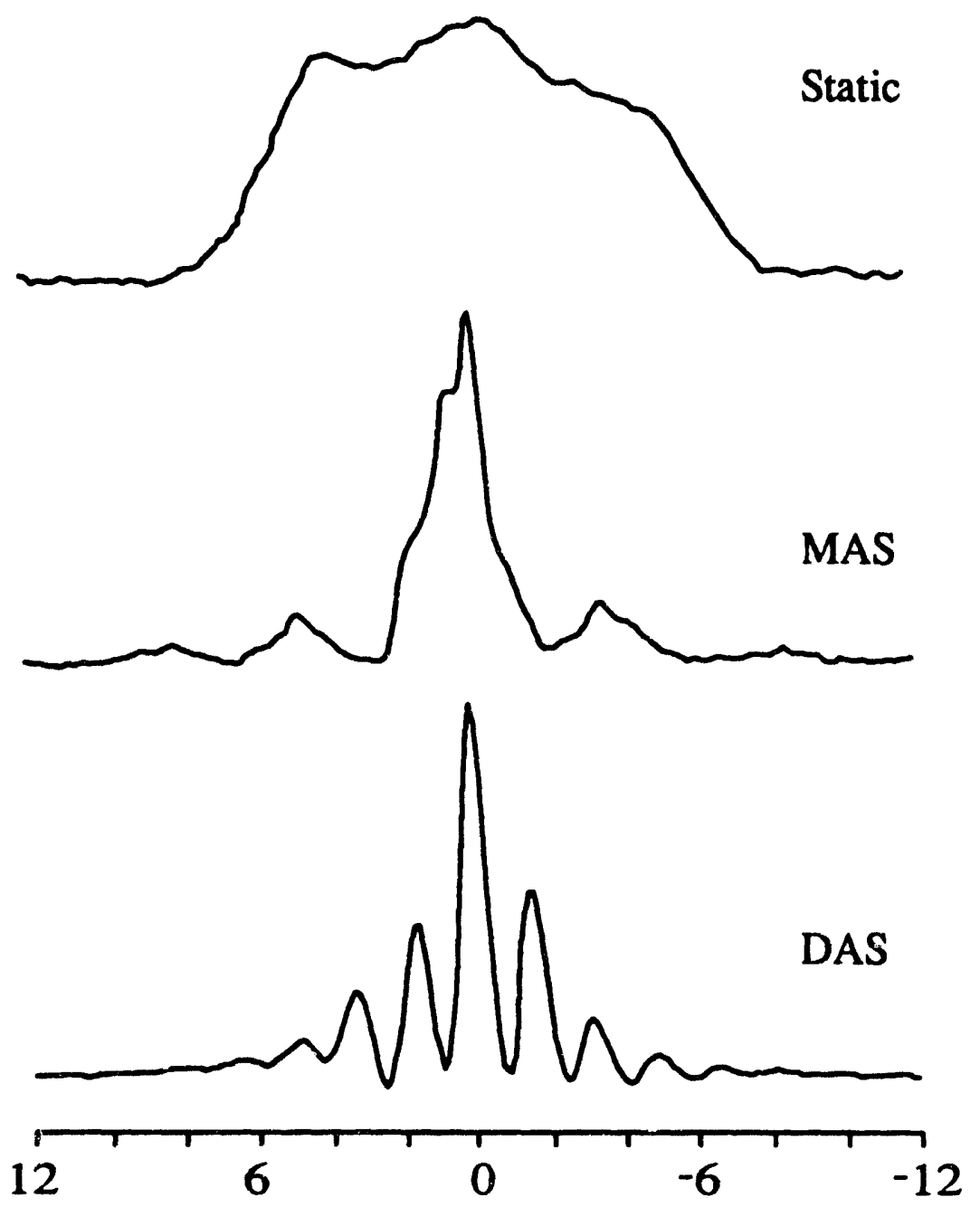

Figure 3.17: Demonstration of DAS experiment with polycrystalline sodium sulfate $\left(\mathrm{Na}_{2} \mathrm{SO}_{4}\right)$, where $\omega_{Q}=433.3 \mathrm{kHz}$ and $\eta=0.6$. (a) Static powder pattern, (b) MAS result, (c) DAS result. The sidebands in the DAS spectrum appears at half of the spinning speed $(3.36 \mathrm{~K} \mathrm{~Hz}$ in this experiment). 


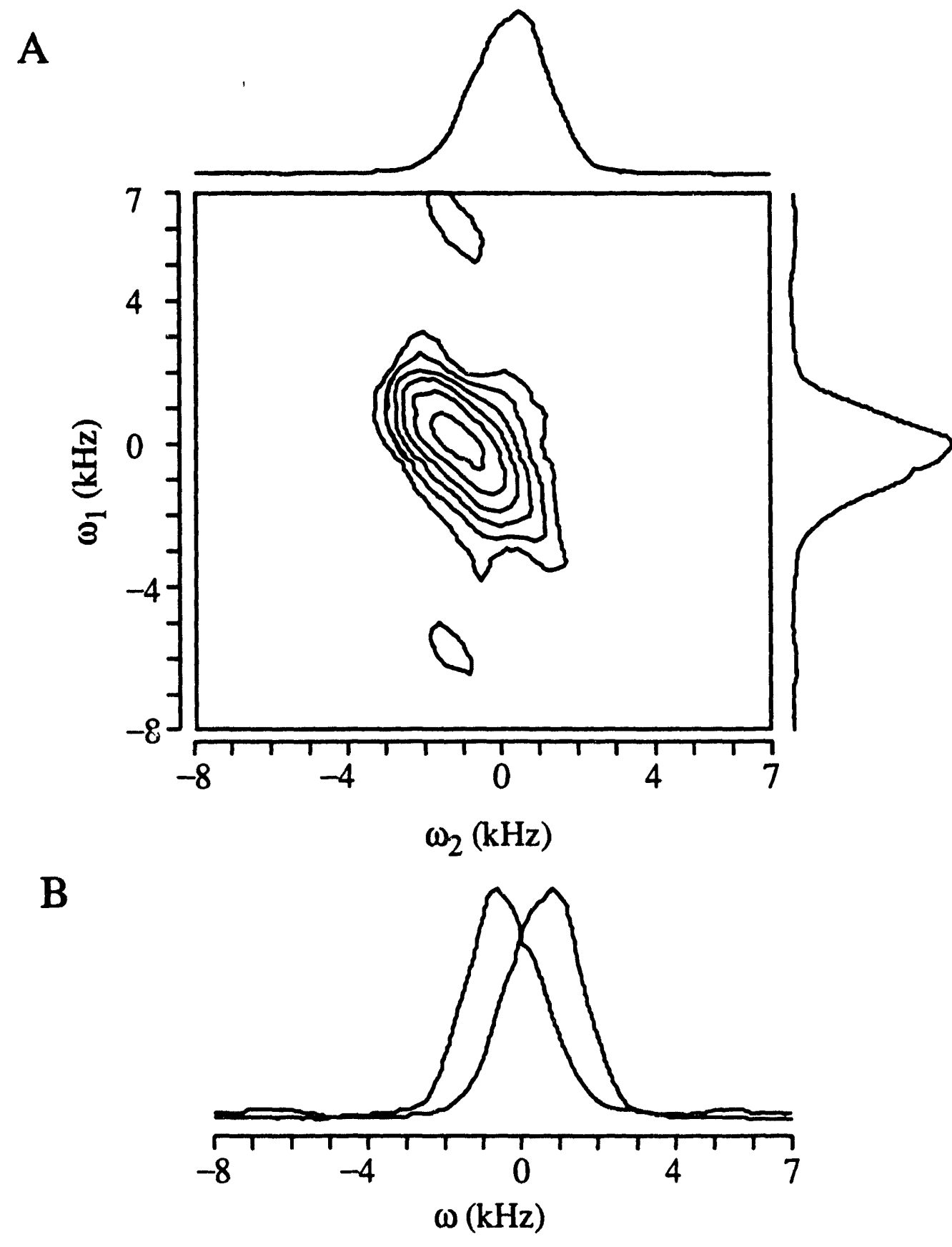

Figure 3.18: (A) Two-dimensional DAS spectrum of polycrystalline sodium oxalate $\left(\mathrm{Na}_{2} \mathrm{C}_{2} \mathrm{O}_{4}\right)$, where $\omega_{Q}=403 \mathrm{kHz}$ and $\eta=0.72$. The projection on the $F_{1}$ domain shows the lineshape spinning at $\theta_{1}$ while the projection on $F_{2}$ domain produces the lineshape spinning at $\theta_{2}$, and (B), the two projected spectra are mirror image with each other. The DAS spectrum yields from the projection along the axis at the angle of $\tan ^{-1} k=45^{\circ}$ with respect to the $F_{2}$ axis. Spinning speed is about $5.5 \mathrm{kHz}$. 
onal components, $I_{x}^{\frac{1}{2} \frac{-1}{2}}$ and $I_{y}^{\frac{1}{2} \frac{-1}{2}}$ in the density matrix. The other components are therefore lost and this leads to broadenings of the signals in the spectra. Indeed, the dipolar Hamiltonian is represented by bilinear spin operators and therefore produces many orders of coherences in the density matrix. Although in principle there is a way to store all these orders in the density matrix, this is just too complicated to implement it experimentally. Another fact is that the rf pulse is actually equivalent to a selective pulse due to the large first order quadrupolar interaction in the Hamiltonian, and therefore can affect only the energy levels corresponding to $m= \pm \frac{1}{2}$. After the dipolar Hamiltonian is expanded according to the fictitious spin operators, there is a scalar term with the form $I_{i}^{\frac{1}{2} \frac{-1}{2}} I_{j}^{\frac{1}{2}} \frac{-1}{2}$ which can not be canceled by any pulse sequences. This may result in the dipolar broadenings in the DAS signals that cannot be eliminated in any way.

For other DAS complementary angles, the experimental set up is same as the one used in Figure (3.14) and the pulse sequence shown in Figure (3.15). The DAS spectrum may then be obtained from the projection along an axis inclined at an angle of $\tan ^{-1} k$ with respect to $F_{2}$ axis in the frequency domain.

\subsection{Powder Lineshape in A Multi-Dimensional Space}

We discuss here the two dimensional lineshapes of the central transitions obtained by flipping the sample between two arbitrary angles. Figure (3.19) shows the variations of the two dimensional powder lineshapes with different flipping angles and different asymmetry parameters. As can be seen, these two dimensional lineshapes consist of a ridge when the asymmetry parameter is zero. This high resolution feature of the powder lineshapes observed in two dimensional space raises a fundamental question whether any powder lineshapes will be highly resolved in a high dimensional space.

To discuss this question, let's consider the lineshapes arising from the chemical 

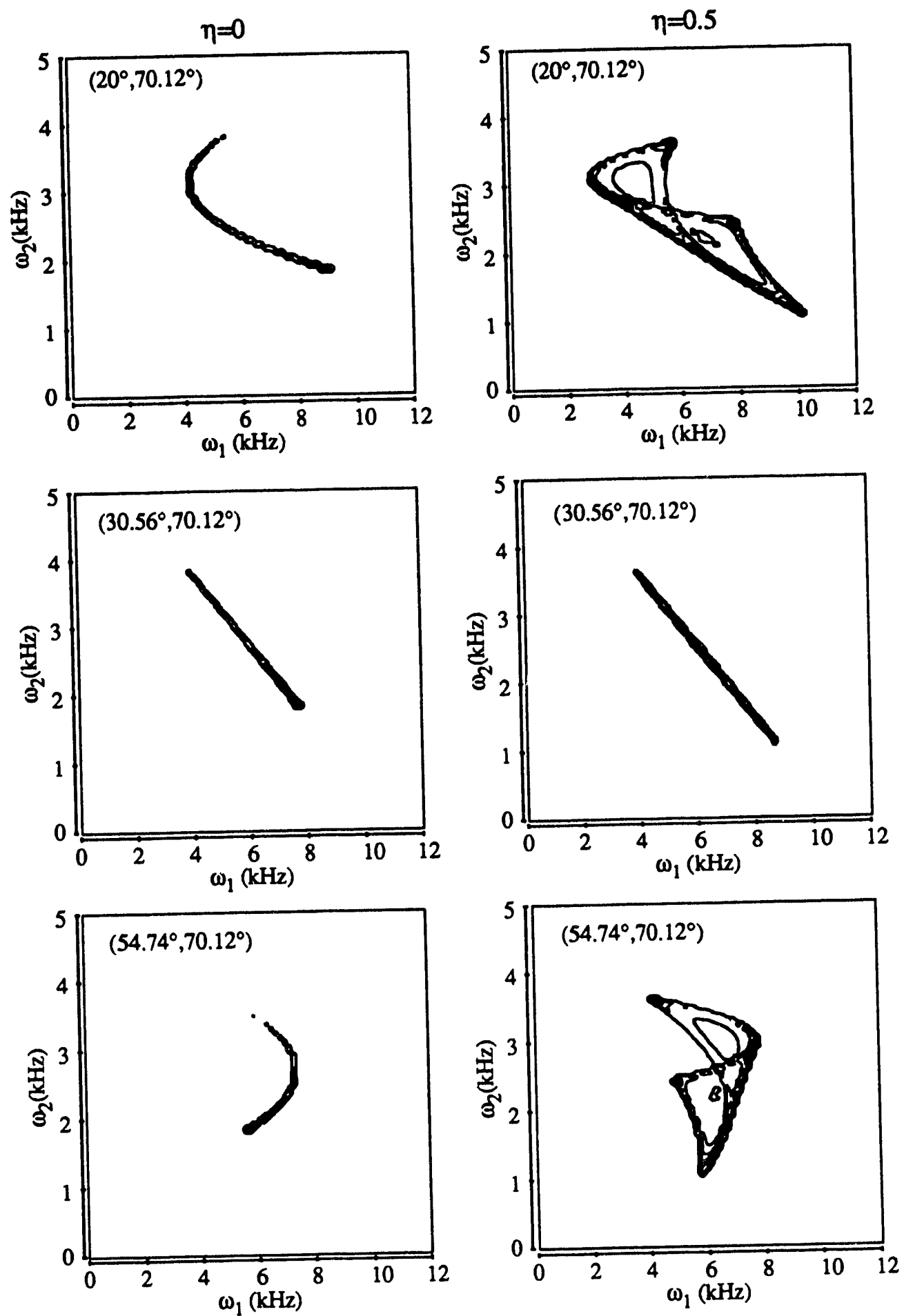

Figure 3.19: Two dimensional lineshapes of central transition with half integer spin nuclei show high resolution features, especially for the $\eta=0$ case. $\theta_{2}$ is $70.12^{\circ}$ for all six graphs, and theta $a_{1}$ is, $\theta_{1}=20^{\circ}$ (top row), $30.56^{\circ}$ (middle), and $54.74^{\circ}$ (bottom) for $\eta=0$ (left column) and 0.5 (right column), where $\omega_{Q}=400 \mathrm{kHz}, \omega_{0}=100 \mathrm{M} \mathrm{Hz}$, and $I=\frac{3}{2}$. 
shift anisotropy interaction. It is well known that the resonance frequency with a chemical shift anisotropy interaction (CSA) for any spin nuclei can be represented as

$$
\omega_{C S}=\omega_{c}\left[\mathcal{D}_{0,0}^{(2)}(\beta)+\frac{1}{2} \eta_{c}\left(\mathcal{D}_{2,0}^{(2)}(\alpha, \beta)+\mathcal{D}_{-2,0}^{(2)}(\alpha, \beta)\right)\right],
$$

where $\omega_{c}$ is the anisotropy of the CSA tensor, and $\eta_{c}$ is the asymmetry parameter. After the sample is subjected to a fast spinning around a fixed axis inclined at the angle, $\theta$, with respect to the external field, $\omega_{C S}$ is scaled down by a factor of $P_{2}(\cos \theta)$, that is

$$
\omega_{C S}(\theta)=P_{2}(\cos \theta) \omega_{C S}(0)
$$

Consider a DAS-type experiment involving only CSA interactions, in which the sample rotates around an axis inclined at $\theta_{1}$ during the evolution time $t_{1}$ and then flips to the second angle $\theta_{2}$ during evolution time $t_{2}$. After implementing a two-dimensional Fourier transformation on the FID signals, the relationship of the frequencies between the first and the second domain is

$$
\omega_{2}=\frac{P_{2}\left(\cos \theta_{2}\right)}{P_{2}\left(\cos \theta_{1}\right)} \omega_{1}+\left[1-\frac{P_{2}\left(\cos \theta_{2}\right)}{P_{2}\left(\cos \theta_{1}\right)}\right] \omega_{i s o}
$$

where $\omega_{i s o}$ is the isotropic shift. Eq.(3.36) tells us that after the powder lineshape determined by Eq.(3.34) is represented in two dimensional space, the contour of the lineshape will be a straight line. The slope of the line is determined by the ratio of $P_{2}\left(\theta_{2}\right) / P_{2}\left(\theta_{1}\right)$. The center of the line is given by the isotropic shift multiplied by $\left[1-\frac{P_{2}\left(\cos \theta_{2}\right)}{P_{2}\left(\cos \theta_{1}\right)}\right]$, and the length of the line relates to the strength of the CSA tensor. Spectra with different isotropic chemical shift will be separated with each other in this two dimensional space, and high resolution one dimensional spectra can therefore be obtained from these projection along the axis at the angle of $\tan ^{-1}\left[\frac{P_{2}\left(\theta_{2}\right)}{P_{2}\left(\theta_{1}\right)}\right]$ with respect to the $f_{1}$ domain. The chemical shift measured by the projection spectra obviously is scaled according to the slope of the contour line, and the anisotropy and asymmetry parameter of the CSA tensor may be obtained from the lineshape along the contour 
line. Another way to obtain two-dimensional spectra is to redefine $P_{2}(\cos \theta) t$ as a new variable and then to linearize this variable by properly choosing the sample spinning axis angle. The two-dimensional FID signal becomes a function of $t$ and $P_{2}(\cos \theta) t$, that is $G\left(t, P_{2}(\cos \theta)\right) t$. After two-dimensional Fourier transformation, one dimension will be high resolution spectra whose peaks correspond to isotropic chemical shifts, and the other dimension shows CSA powder lineshapes. This experiment is called MYDAS proposed by L. Frydman and his co-workers [89].

In the case of the second order effects of the quadrupolar interactions, the twodimensional powder lineshapes of the central transitions become ambiguous as can be seen from Figure (3.19). Only for $\eta=0$, the contour of the lineshape keeps the high resolution structure in two dimensional space and only when the two angles satisfies condition Eq.(3.25), the contour line is straight (of course this case is still true for any asymmetry parameter). For other angles, powder lineshapes show a smooth curve. From Eq.(3.29), under the fast spinning condition around an axis inclined at the angle of $\theta$ with respect to the external field, the second order shift for central transition is

$$
\omega_{\frac{1}{2} \leftrightarrow-\frac{1}{2}}^{(2)}(\theta)=C_{2} P_{2}(\theta) A_{2,0}+C_{4} P_{4}(\theta) A_{4,0}
$$

where $C_{l}$ is given in Eq.(3.28) and $P_{l}(\theta)$ is the $l$-th order Legendre polynomial. From Eq. (3.13), the angular dependence of $A_{l, 0}$ is only determined by the Legendre polynomials, that is,

$$
A_{l, 0}=P_{l}(\beta) \rho_{l, 0},
$$

where $\rho_{l, 0}$ is given in Eq.(3.12). The contour of the two dimensional lineshapes of the central transition is therefore determined by the solution of the following two simultaneous equations.

$$
\begin{aligned}
& \omega_{1}=C_{2} \rho_{2,0} P_{2}\left(\theta_{1}\right) P_{2}(\beta)+C_{4} \rho_{4,0} P_{4}\left(\theta_{1}\right) P_{4}(\beta) \\
& \omega_{2}=C_{2} \rho_{2,0} P_{2}\left(\theta_{2}\right) P_{2}(\beta)+C_{4} \rho_{4,0} P_{4}\left(\theta_{2}\right) P_{4}(\beta) .
\end{aligned}
$$


The solution of this equation is

$$
\begin{aligned}
& \omega_{2}=B+k \omega_{1}+A \sqrt{C+D \omega_{1}} \text { for } \omega_{\min } \leq \omega_{1} \leq \omega_{\text {mid }} \\
& \omega_{2}=B+k \omega_{1}-A \sqrt{C+D \omega_{1}} \text { for } \omega_{\min } \leq \omega_{1} \leq \omega_{\max }
\end{aligned}
$$

where

$$
\begin{aligned}
k & =\frac{P_{4}\left(\theta_{2}\right)}{P_{4}\left(\theta_{1}\right)} \\
k^{\prime} & =\frac{C_{2} \rho_{2,0} P_{2}\left(\theta_{1}\right)}{C_{4} \rho_{4,0} P_{4}\left(\theta_{1}\right)} \\
A & =\frac{3}{35} C_{2} \rho_{2,0}\left[P_{2}\left(\theta_{2}\right)-P_{2}\left(\theta_{1}\right) k\right] \\
B & =\frac{4}{3}\left(5-9 k^{\prime}\right) A \\
C & =30-10 k^{\prime}+9 k^{\prime 2} \\
D & =\frac{70}{C_{4} \rho_{4,0} P_{4}\left(\theta_{1}\right)} \\
\omega_{\min } & =\frac{1}{7}\left[C_{2} \rho_{2,0} P_{2}\left(\theta_{1}\right)-3 C_{4} \rho_{4,0} P_{4}\left(\theta_{1}\right)\right]+\frac{9 k^{\prime 2}}{D}+C_{0} \rho_{0,0} \\
\omega_{\text {mid }} & =-\frac{1}{2} C_{2} \rho_{2,0} P_{2}\left(\theta_{1}\right)+\frac{3}{8} C_{4} \rho_{4,0} P_{4}\left(\theta_{1}\right)+C_{0} \rho_{0,0} \\
\omega_{\text {max }} & =C_{0} \rho_{0,0}+C_{2} \beta_{2,0} P_{2}\left(\theta_{1}\right)+C_{4} \rho_{4,0} P_{4}\left(\theta_{1}\right) .
\end{aligned}
$$

In the above equations, we assume the isotropic frequency is zero; otherwise the frequency variables have to be replaced by $\omega_{i}-\omega_{i s o}$. In Eq. (3.40), $\omega_{2}$ is doublevalued only in the region $\omega_{\min } \leq \omega_{1} \leq \omega_{\operatorname{mid}}$, where the $\omega_{\min }, \omega_{\operatorname{mid}}$, and $\omega_{\max }$ are the frequencies of the singularities observed in the one dimensional powder lineshapes (see Figure (3.2) and Table (3.4)). The curvature of the contour may be obtained from the derivative of Eq.(3.40). Since the coefficient $D$ depends on the anisotropy of the quadrupolar tensor, the curvature of the contour will in general depend on the anisotropy except when $A=0$, which is again the DAS trajectory. This means that although the lineshape of the central transition with $\eta=0$ is highly resolved in two dimensional space, it is not easy to obtain a projection spectrum in which the position of peaks only relates to the isotropic shift of the spin nuclei. However the 
extraction of the anisotropic information still may be implemented by simulating the two dimensional contour line by use of Eq.(3.40).

To overcome the ambiguity of these two dimensional lineshapes of the central transitions, it is necessary to extend the dimensionality of the observing space. As we will see, the lineshapes will, indeed, become resolved planes in three dimensional space. Actually, from Eq.(3.37), the resonance frequencies including isotropic shifts in tree dimensional space are

$$
\begin{aligned}
& \omega_{1}-\omega_{\text {iso }}=C_{2} P_{2}\left(\theta_{1}\right) A_{2,0}+C_{4} P_{4}\left(\theta_{1}\right) A_{4,0} \\
& \omega_{2}-\omega_{\text {iso }}=C_{2} P_{2}\left(\theta_{2}\right) A_{2,0}+C_{4} P_{4}\left(\theta_{2}\right) A_{4,0} \\
& \omega_{3}-\omega_{\text {iso }}=C_{2} P_{2}\left(\theta_{3}\right) A_{2,0}+C_{4} P_{4}\left(\theta_{3}\right) A_{4,0} .
\end{aligned}
$$

It is easy to obtain the solution of Eq.(3.42) which is

$$
\omega_{1}=A^{\prime} \omega_{2}+B^{\prime} \omega_{3}+\left(1-A^{\prime}-B^{\prime}\right) \omega_{i s o}
$$

where

$$
\begin{aligned}
A^{\prime} & =\frac{P_{2}\left(\theta_{1}\right) P_{4}\left(\theta_{3}\right)-P_{2}\left(\theta_{1}\right) P_{4}\left(\theta_{1}\right)}{P_{2}\left(\theta_{2}\right) P_{4}\left(\theta_{3}\right)-P_{2}\left(\theta_{3}\right) P_{4}\left(\theta_{2}\right)} \\
B^{\prime} & =\frac{P_{2}\left(\theta_{2}\right) P_{4}\left(\theta_{1}\right)-P_{2}\left(\theta_{1}\right) P_{4}\left(\theta_{2}\right)}{P_{2}\left(\theta_{2}\right) P_{4}\left(\theta_{3}\right)-P_{2}\left(\theta_{3}\right) P_{4}\left(\theta_{2}\right)} .
\end{aligned}
$$

Eq.(3.43) defines a plane in the three dimensional space, and the coefficients, $A^{\prime}$ and $B^{\prime}$, are related only to the angles of the three sample spinning axes. The center of the plane is determined by the isotropic shift multiplied by a factor of $\left(1-A^{\prime}-B^{\prime}\right)$ and the area of the plane is related to the strength of the quadrupolar interaction.

\subsection{Double Rotation (DOR)}

In the sections 3.5 and 3.6 , we have described the principles and the experiments of the dynamic angle spinning (DAS). Now we turn to discuss another main method for obtaining high resolution spectra for central transition with quadrupolar interaction, 
named double rotation (DOR). After applying a rotation around a fixed axis at a particular angle with respect to the external field to, the Hamiltonian will be truncated along the rotation axis if the spinning speed is fast enough. The residual parts then are determined by the Legendre polynomial $P_{l}(\cos \theta)$ (in our case, $l=2,4$ ). For example, if the applied rotation is MAS, the residual lineshape of the central transition for a powder sample is uniquely determined by the fourth order Legendre polynomial, $P_{4}(\cos \theta)$. The linewidth of the lineshape will be scaled down by a factor of $1 / P_{4}\left(\cos \theta_{m}^{(2)}\right)=2.57$ where $\theta_{m}^{(2)}=54.74^{\circ}$, the magic angle of the second order Legendre polynomial $P_{2}(\cos \theta)$. In the variations of $P_{2}(\cos \theta)$ and $P_{4}(\cos \theta)$ with $\theta$, there is no cross point at which the values of $P_{2}(\cos \theta)$ and $P_{4}(\cos \theta)$ are equal to zero simultaneously (see Figure 3.8). However, the linewidth of the powder pattern for a powder sample varies as the angle of the rotation axis changes (see Figure 3.9 ), and can be further narrowed by applying another rotation relative to the first rotation. In other words, the linewidths of the powder patterns can be continuously narrowed to any degree by properly applying several rotations in same time. A trivial solution can be found to eliminate completely the second order broadening with two rotations. This corresponds to the double rotation (DOR) method: the first rotation is performed along the magic angle $\theta_{m}^{(2)}$ with respect to the external magnetic field while the second rotation occurs at one of the magic angles $\theta_{m}^{(4)}$ of the fourth order Legendre polynomial $P_{\mathbf{4}}(\cos \theta)$.

After applying two rotations to Eq.(3.20) and by using Eq.(3.12) twice, the transition frequency between two central levels $\left(m= \pm \frac{1}{2}\right)$ becomes

$$
\omega_{\frac{1}{2} \leftrightarrow-\frac{1}{2}}^{(2)}(t)=\sum_{l=0,2,4} C_{l} \sum_{m_{1}, m_{2}=-l}^{l} \mathcal{D}_{m_{1}, 0}^{(l)}\left(\Omega_{r_{1}}(t)\right) \mathcal{D}_{m_{2}, m_{1}}^{(l)}\left(\Omega_{r_{2}}(t)\right) A_{l m_{2}}
$$

where $C_{l}$ is given by Eq.(3.26) and $\Omega_{r_{1}}(t), \Omega_{r_{2}}(t)$ are two sets of Euler angles which define the transformations from the laboratory frame to the outer rotor frame (ORF) (the first rotor frame) and from the outer rotor frame to the inner rotor frame (IRF) (the second rotor frame) respectively. We assume that the spinning speeds of the 
outer and inner rotors are $\omega_{r_{1}}$ and $\omega_{r_{2}}$, the first rotation axis angle is $\beta_{r_{1}}$ with respect to the external field while the second rotation axis is at the angle of $\beta_{r_{2}}$ relative to the first rotation axis. The initial phases of the first and second rotations are $\gamma_{r_{1}}$ and $\gamma_{r_{2}}$ respectively. The explicit expression of the two sets of Euler angle are therefore given by

$$
\left\{\begin{array}{l}
\Omega_{r_{1}}=\left(\omega_{r_{1}} t+\alpha_{r_{1}}, \beta_{r_{1}}, \gamma_{r_{1}}\right) \\
\Omega_{r_{2}}=\left(\omega_{r_{2}} t+\alpha_{r_{2}}, \beta_{r_{2}}, \gamma_{r_{2}}\right)
\end{array}\right.
$$

As in MAS, all spins have cylindrical symmetry around the external magnetic field $H_{0}$ so that $\gamma_{r_{1}}$ can be set to zero. For a powder sample, the absolute orientation of individual spin in the IRF is not important because spins have equal probability to be at each orientation. Thus we can also set $\alpha_{r_{2}}$ to zero. Finally, $\alpha_{r_{1}}$ and $\gamma_{r 2}$ determine the relative phase $\gamma_{r}$ between outer and inner rotors (say $\gamma_{r}=\alpha_{r_{1}}+\gamma_{r_{2}}$, see Figure (3.20)).

After expanding Eq.(3.45), three terms result: the first term $(l=0)$ is a scalar, independent of the orientation and of time; the second term is only time independent; and the third part depends on both orientation and time. Furthermore since the Hamiltonian commutes with itself at all time, the total phase evolved after application of a $90^{\circ}$ pulse is

$$
\varphi(t)=C_{0} A_{0,0} t+\sum_{l=2,4} C_{l} \omega_{l} t+\sum_{l=2,4} C_{l} \varphi_{l}(t)
$$

where

$$
\omega_{l}=\sum_{m=-l}^{l} d_{m, 0}^{(l)}\left(\beta_{r_{1}}\right) d_{-N m, m}^{(l)}\left(\beta_{r_{2}}\right) A_{l,-N m}
$$

and

$$
\varphi_{l}(t)=\sum_{m_{1}, m_{2} \neq 0} \frac{\imath d_{m_{1}, 0}^{(l)}\left(\beta_{r_{1}}\right) d_{m_{2}, m_{1}}^{(l)}\left(\beta_{r_{2}}\right)}{m_{1} \omega_{r_{1}}+m_{2} \omega_{r_{2}}} A_{l m_{2}} e^{-\imath m_{1} \gamma_{r}}\left(e^{-\imath\left(m_{1} \omega_{r_{1}}+m_{2} \omega_{r_{2}}\right) t}-1\right)
$$

and $N=\frac{\omega_{r_{1}}}{\omega_{r_{2}}}$. The first term in Eq.(3.49) corresponds to the isotropic shift, the second term determines the linewidth and also the lineshape for powder samples, and the third term generates a set of sidebands. 


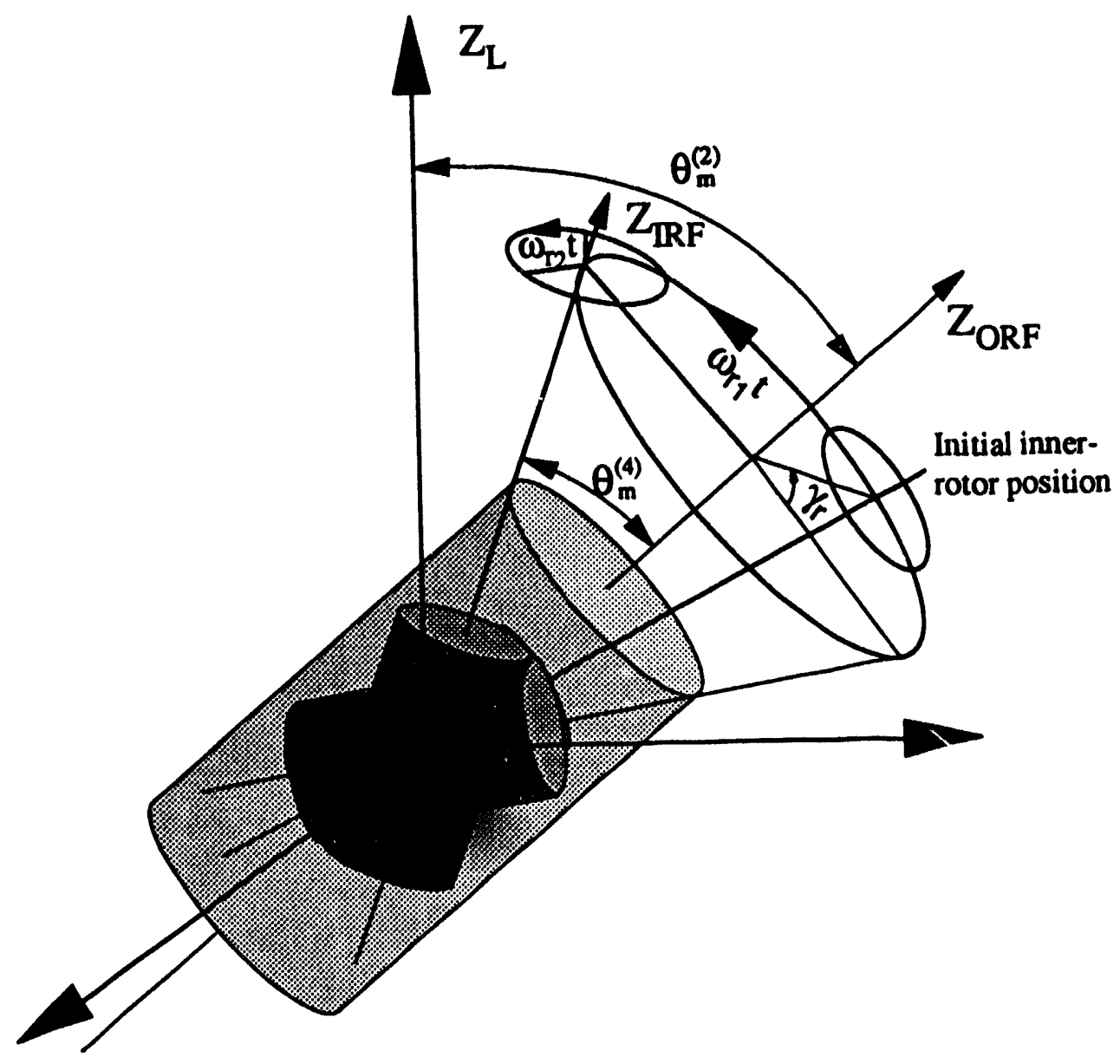

Figure 3.20: Scheme of the coordinate transformation among the laboratory frame, the outer rotor frame, and the inner rotor frame. The rotation axis of the outer rotor is titled at the magic angle, $\theta_{m}^{(2)}=54.74^{\circ}$, of the second order Legendre polynomial, while the inner rotor spins around $\theta_{m}^{(4)}=30.56^{\circ}$, one of the magic angles of the fourth order Legendre polynomial. 
If we ssume that the spinning speeds of both rotations are much larger than the amplitudes of the spin Hamiltonian $\mathcal{H}^{(1)}$ of the central transition, the third term in Eq. (3.49), $\varphi_{l}(t)$, becomes small enough to be neglected at all time. Since from the properties of Wigner rotation matrices, $d_{0,0}^{(l)}(\beta)=P_{l}(\cos (\beta))$, the anisotropic shift in Eq.(3.49) $\omega_{l^{\prime}}$ can be eliminated if

$$
\left\{\begin{array}{l}
P_{2}\left(\cos \beta_{r_{1}}\right) P_{2}\left(\cos \beta_{r_{2}}\right)=0 \\
P_{4}\left(\cos \beta_{r_{1}}\right) P_{4}\left(\cos \beta_{r_{2}}\right)=0
\end{array}\right.
$$

and $N=\frac{\omega_{r_{1}}}{\omega_{r_{2}}}$ is not an integer less than five. The solution of Eq.(3.50) is $\beta_{r_{1}}=$ $\theta^{(2)}=54.74^{\circ}$ and $\beta_{r_{2}}=\theta^{(4)}=30.56^{\circ}, 70.12^{\circ}$, or vice versa. If $N$ is an integer, the residual broadening cannot be totally suppressed due to the interference between two spinning speeds. Figure (3.21) shows the variation of the residual line broadenings in the central transition of a powder sample after application of DOR, for different ratios $N$. From this figure it can be seen that when the two spinning speeds are equal, $N=1$, the linewidth of centerband is about one tenth of the static linewidth, and will narrow with increasing ratios between the two spinning speeds. The linewidth reaches the order of the relaxation broadening, or of high order residual broadenings when the ratio between the two spinning speed is larger than three. This means that the condition we gave at the beginning is not very crucial and therefore the design of a double-rotor probe should concentrate on increasing the spinning speed of the outer rotor. When the ratio between the two spinning speeds are not an integer, the lineshapes shown in Figure (3.21) will be the envelop of the sidebands which come from the interference between the two spinning speeds.

\subsection{DOR Experiments}

DOR experiments were carried out on Bruker $\mathrm{AM}-400 \mathrm{MHz}$ spectrometer with a homemade DOR probe $[79,104]$. The static magnetic field was shimmed using the deuterium resonance of $\mathrm{D}_{2} \mathrm{O}$; linewidths are below $10 \mathrm{~Hz}$. 


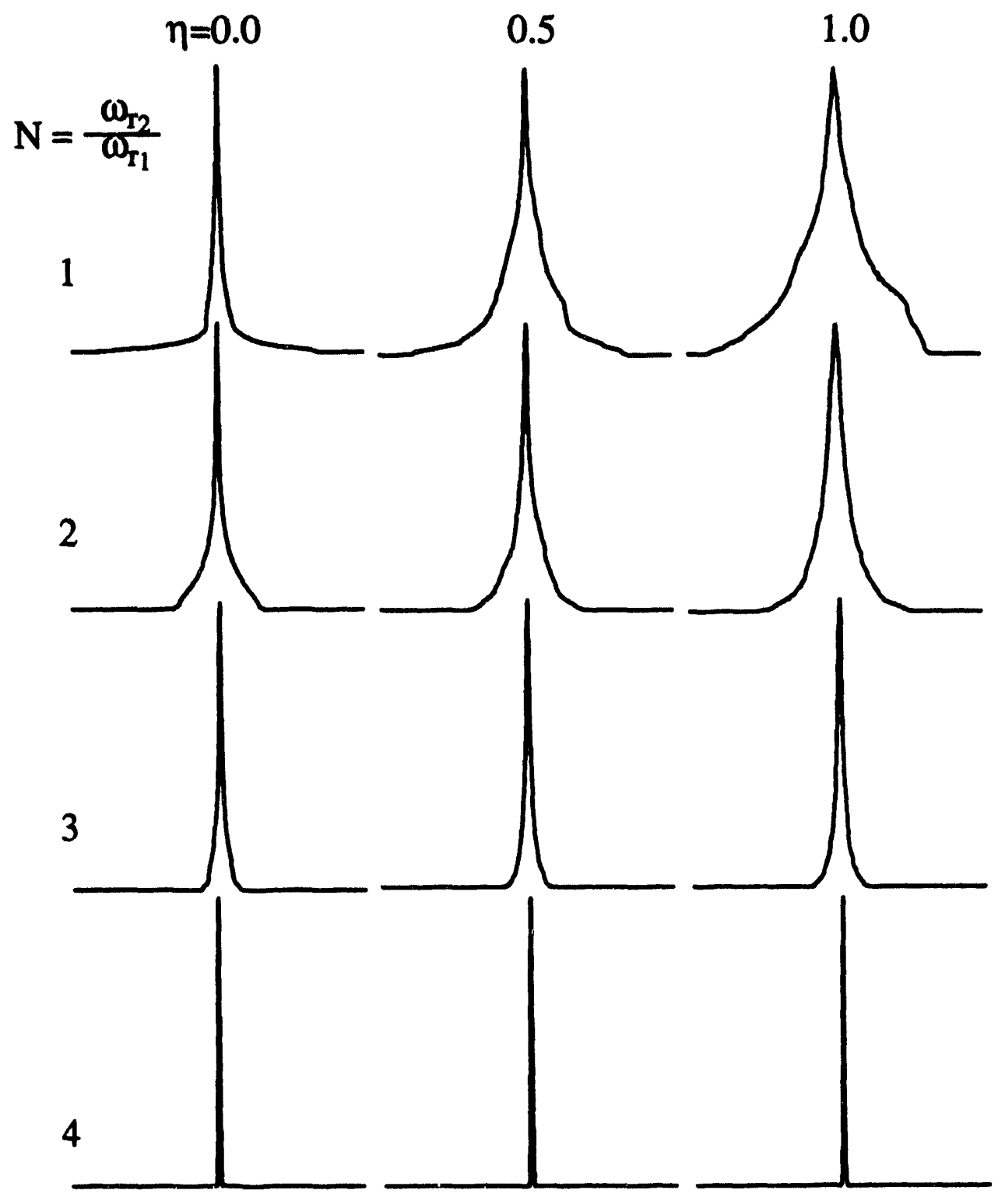

Figure 3.21: Residual lineshapes of the centerband of the central transition with quadrupolar coupling constant $2.6 \mathrm{MHz}$ of spin $I=\frac{3}{2}$ nuclei after double rotation (DOR) varying with the ratio of the two spinning speeds, $\frac{\omega_{r_{2}}}{\omega_{r_{1}}}$, and the asymmetry parameter $\eta$. 
In the DOR probe[79, 104], the sample is contained in a small rotor which is embedded in the body of a large rotor spinning at the magic angle $\beta_{1}=54.74^{\circ}$ of the second order Legendre polynomial with respect to the static magnetic field. Henceforth, the small rotor is named inner rotor and the large rotor as outer rotor. The angle $\beta_{2}$ between the spinning axes of the inner and outer rotors is $30.56^{\circ}$, one of the magic angles of the fourth order Legendre polynomial. To design an efficient double-rotor many of the requirements for a conventional high performance MAS system have to be met[91]. A further complication with double-rotor systems arises from the fact that a spinning object, like the inner rotor, has a tendency to maintain its spinning orientation unless a torque is exerted on it. Torque is obviously not desirable here because it imposes an extra burden on the limited load capacity of bearing system. Fortunately, as will be illustrated below, the torque can be reduced to a tolerably small value when the ratio of the two spinning speeds, $\omega_{2} / \omega_{1}$, approaches a certain fixed value that depends on the structure of the inner rotor. As illustrated in Figure (3.22), the motion of the inner rotor can be visualized as the motion of a rigid cylinder with a fixed point, in this case the cross-point of the two rotation axes; the pattern of the motion is the well-known precession motion but without nutation. The equation relevant to such motion is

$$
\frac{d \mathbf{J}}{d t}=\boldsymbol{\tau}
$$

where $\mathbf{J}$ is the angular momentum of the inner-rotor and $\tau$ is the torque applied to the inner rotor through the bearing. The angular momentum can be expressed in terms of the spinning speed $\boldsymbol{\omega}$ as

$$
\mathbf{J}=\mathbf{i} I_{x} \omega_{x}+\mathbf{j} I_{y} \omega_{y}+\mathbf{k} I_{z} \omega_{z}
$$

where $\mathbf{i}, \mathbf{j}$ and $\mathbf{k}$ are the unit vectors of the principal axis system $(x, y, z)$ of the moment of inertia tensor of the inner-rotor with principal values $I_{x}, I_{y}$ and $I_{z}$. In our case this tensor is symmetric, $I_{x}=I_{y}$. There are two contributions to the inner-rotor 


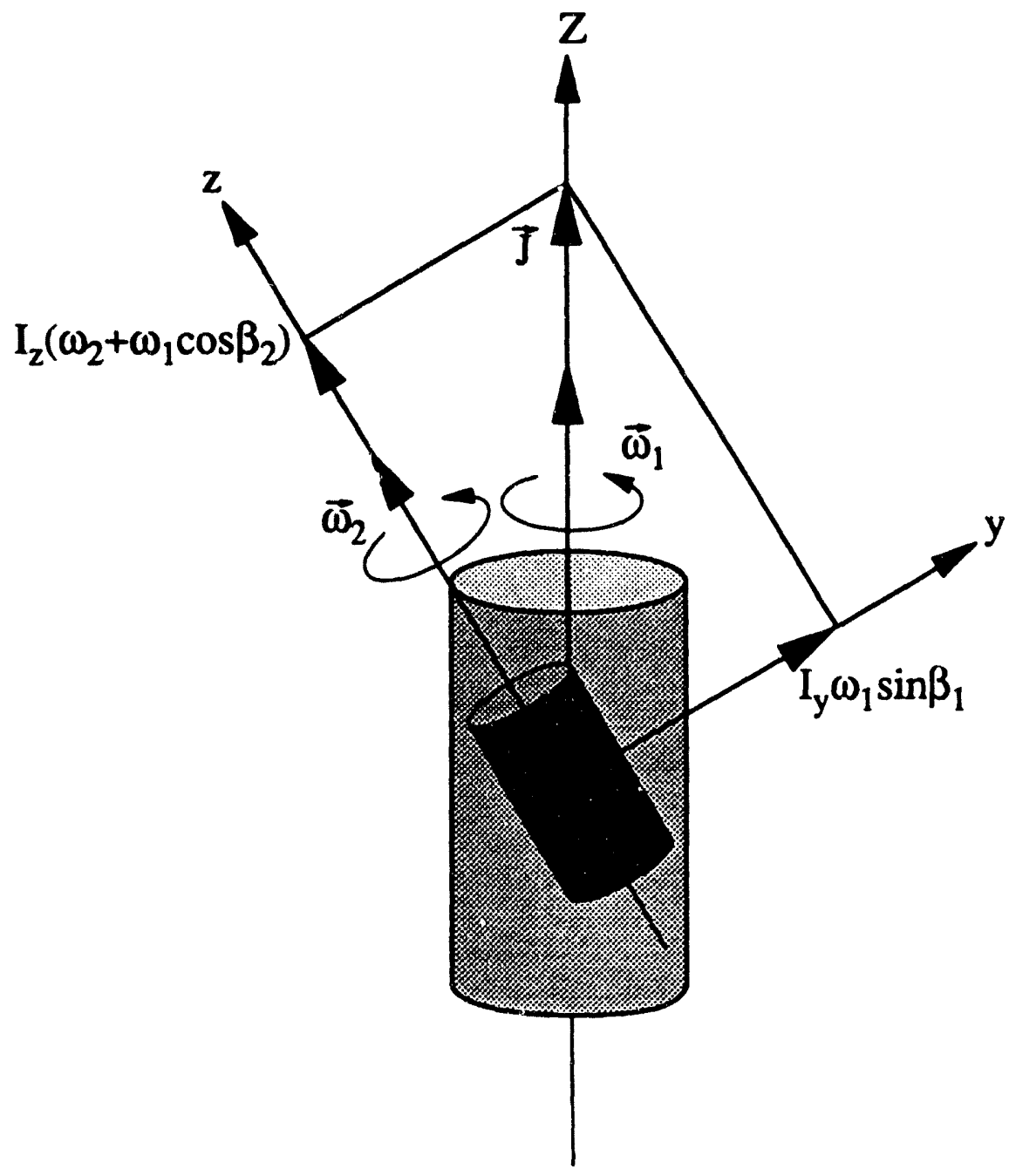

Figure 3.22: By adjusting the ratio of moments of inertia $\left(I_{y} / I_{z}\right)$, the addition of the two vector components of the angular momentum along the inner-rotor axis $z$ and the $y$ axis can be made to poirit along the outer-rotor axis $Z$, so that spinning the outer-rotor will not affect the orientation of the total angular momentum of the inner-rotor. 
spinning speed, the spinning speed of the inner-rotor, $\omega_{2}$, spinning around its own axis and the spinning speed $\omega_{1}$ imposed by the outer-rotor. This second contribution can be decomposed into two components, $\omega_{1} \cos \beta_{2}$ along the z-axis, the spinning axis of the inner-rotor, and $\omega_{1} \sin \beta_{2}$ along the $y$-axis which is chosen to be in the plane, $\mathrm{zZ}$, where the $\mathrm{Z}$ direction is along the outer-rotor spinning axis. Thus we have

$$
\omega_{x}=0, \quad \omega_{y}=\omega_{1} \sin \beta_{2}, \quad \omega_{z}=\omega_{2}+\omega_{1} \cos \beta_{2}
$$

Viewed from the laboratory frame, the only way to make $\mathrm{J}$ time-independent (i.e. zero torque) is to design the system in such a way that $\mathbf{J}$ points along the $\mathbf{Z}$-axis as depicted in Figure (3.22). This can be achieved if

$$
\tan \beta_{2}=\frac{I_{y} \omega_{2} \sin \beta_{2}}{I_{z}\left(\omega_{1}+\omega_{2} \cos \beta_{2}\right)}
$$

or

$$
\omega_{2} / \omega_{1}=\cos \beta_{2}\left(\frac{I_{y}}{I_{z}}-1\right) \equiv k
$$

Clearly, from this equation, for a fixed ratio of $I_{y} / I_{z}$, determined by the dimensions of the inner-rotor, the ratio $\omega_{2} / \omega_{1}$ is fixed for torque-free conditions.

For a ratio $\omega_{2} / \omega_{1}$ of 5 , when the outer rotor speed reaches $1 \mathrm{kHz}$, the inner-rotor speed must be $5 \mathrm{kHz}$; this is not hard to achieve with current techniques. Since the inner rotor is inclined at an angle inside the outer-rotor, the dimensions of the inner rotor (both the length and the diameter) will determine the eventual diameter of the outer rotor which is a crucial factor in obtaining high outer rotor speeds. With the goal of producing higher speeds for both the inner and outer rotors, we have designed a new double-rotor[104] with a reduced diameter of the outer rotor and a turbine system for the inner rotor with parameters recommended for MAS operation[91]; the driving jets are placed at both ends of the inner rotor.

An illustrative sketch of the double-rotor probe is shown in Figure (3.23). For the purpose of detailed studies of the inner rotor behavior, we used a two-port air 


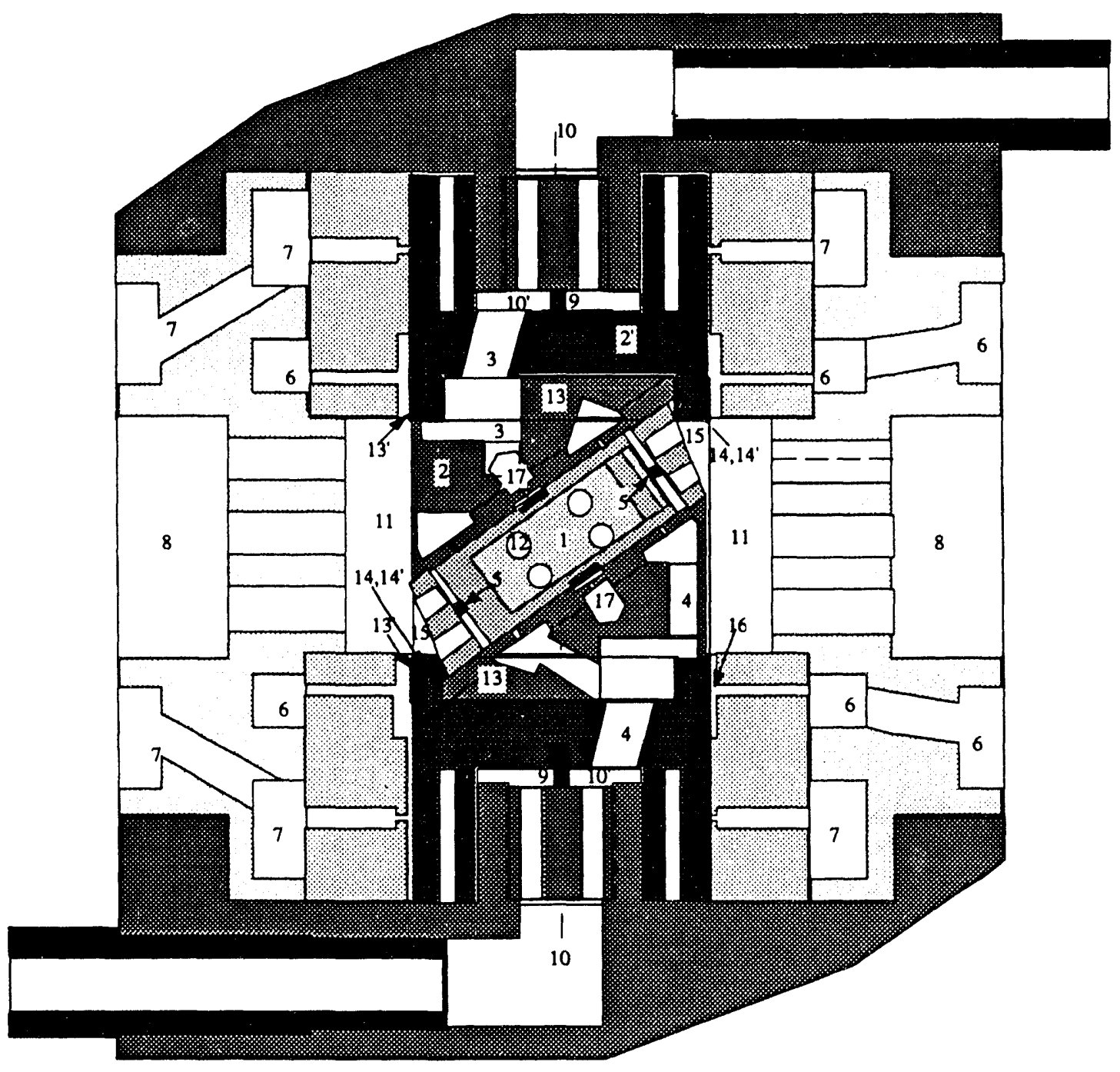

Figure 3.23: New double-rotor probe designs. (1) inner rotor with flutes at both ends; (2 and 2') building blocks of the double rotor held together through the axles (13) with the matched counter parts $\left(13^{\prime}\right)$, and the step-shoulder (14) with the matched counter parts (14'); (3) and (4) air channels and holes for bearing and drive system of the inner rotor; (5) pins to hold the inner rotor in place; (6) and (7) bearing and drive system for the outer rotor; (8) air exhaust holes for the outer rotor; (9) pins to hold the outer rotor in place; (10) air passage for the inner rotor which fits into (10') with small clearance; (11) space for rf coil; (12) air exhaust holes for the inner rotor; (15) caps holding inner-rotor in place and allowing air escape for the inner rotor system; (16) flutes for outer rotor; (17) flutes for inner rotor. 
injection system similar to that used in the original design[79]. The new doublerotor is machined in three pieces held together with the axle (part 13) and the stepshoulders (part 14) on the center piece (part 2) by press-fitting into the matched counter parts (parts $13^{\prime}$ and $14^{\prime}$ ) on the end pieces (part $2^{\prime}$ ). This structure has several advantages. The cylindrical structure of the center piece allows efficient use of the available space within the outer rotor. Moreover, the cylindrical shape also prevents structural deformations; deviations less than $0.015 \mathrm{~mm}$ can be made over the whole length of the outer rotor. In addition, we can now also use more fragile ceramic materials for the rotor body because a tight press-fit of the matching pieces is not necessary here; we can use clamp buttons screwed on top of the axles (part 13) to hold the three pieces together as well. The new structure also prevents any air leakages through mismatched fits. As a result of these modifications, and reducing the inner rotor diameter to $4.6 \mathrm{~mm}$, we were able to reduce the outer-rotor to a diameter of $13 \mathrm{~mm}$ and a length of $46 \mathrm{~mm}$. A further reduction of length and diameter is possible with an one-port air injection system feeding both the bearing and the drive of the inner rotor. The outer rotor described above is able to reach a spinning speed of $2 \mathrm{kHz}$ after careful balancing under conditions of single rotation; a spinning inner rotor has no appreciable influence on the outer-rotor performance when the outer-rotor is spinning below $1 \mathrm{kHz}$.

To operate the double rotor, we start spinning the inner rotor first and then the outer rotor. Initially, the ratio $\omega_{2} / \omega_{1}$ is usually too high to satisfy the torque free condition discussed above. Consequently, a torque is generated, and the friction on the inner rotor from the bearing system slows down the spinning of the inner rotor; at the same time, the ratio $\omega_{2} / \omega_{1}$ decreases and with it the torque and friction, finally stabilizing the inner rotor spinning speed at a lower value that depends on the bearing and drive system. As the outer rotor speed increases, the inner rotor speed must also increase in order to adapt itself to the torque-free condition. In fact, precisely 
at the torque free condition the system is not stable, since any accidental slow-down of the inner rotor can trigger a torque avalanche. To be more specific, any decrease of $\omega_{2}$ increases the torque when the ratio $\omega_{2} / \omega_{1}$ is at the torque free condition; this in turn further decreases $\omega_{2}$ implying a further increase of the torque and leading finally to a crash of the inner rotor motion; the outer rotor system is too large for $\omega_{1}$ to adapt itself quickly to any change of the inner rotor motion. On the contrary, the system is in a stable state when the ratio $\omega_{2} / \omega_{1}$ is close, but higher than the torque free condition, as long as the weak torque generated can be tolerated by the bearing system of the inner rotor. Any accidental slow-down of the inner rotor will now decrease the torque and friction, bringing $\omega_{2}$ up again; similarly, any accidental speed-up of the inner rotor will increase the torque bringing $\omega_{2}$ back down again. This self adjusting mechanism protects the inner rotor against any instabilities. It can be shown[92] that under the influence of a torque the relationship between $\omega_{2}$ and $\omega_{1}$ can be written as

$$
\omega_{2}=\cos \beta_{2}\left(I_{y} / I_{z}-1\right) \omega_{1}+\frac{\tau}{I_{z} \omega_{1} \sin \beta_{2}} \equiv k \omega_{1}+\Delta \omega_{2}
$$

which reduces to Eq.(3.55), the torque free condition, when $\tau$ (the absolute magnitude of $\tau$ in Eq.(3.51)) or $\Delta \omega_{2}$ is set to zero.

Both the inner and outer rotor spinning speeds can be measured experimentally from the sideband positions of ${ }^{23} \mathrm{Na}$ in sodium chloride where the line is broadened by dipole-dipole interactions. The sidebands resulting from motions of the inner and the outer rotors can be clearly distinguished owing to the large $\omega_{2} / \omega_{1}$ ratio which adiabatically decouples the NMR effects of the two rotors. Figure (3.24) is a plot of $\nu_{2}=\omega_{2} / 2 \pi$ versus $\nu_{1}=\omega_{1} / 2 \pi$ obtained for two different inner rotors; $A$ is $13.3 \mathrm{~mm}$ long and $B$ is $15.2 \mathrm{~mm}$ long with $k$ values of 4.5 and 5.1 respectively. The different specific gravities of Vespel and sodium chloride have been taken into account in the calculation of $k$. Points in Figure (3.24) represented by the same kind of solid symbols are obtained under the same conditions for the inner rotor, i.e. the same 


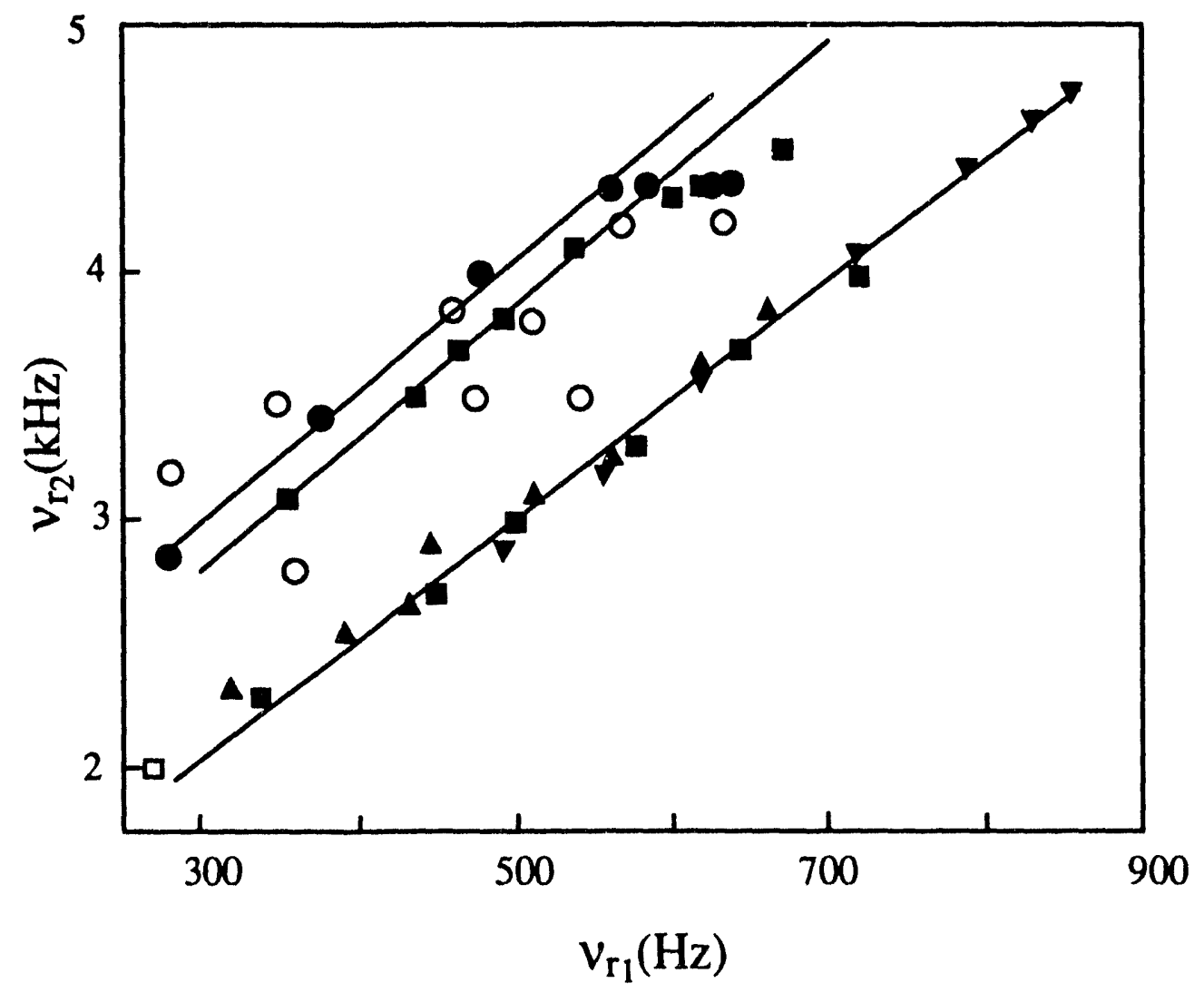

Figure 3.24: The inner rotor spinning speed $\nu_{1}$ is plotted versus the outer rotor spinning speed $\nu_{2}$. The symbols $\Delta, \square$ and $\nabla$ represent data for inner-rotor $A$ (see text) obtained with air pressures of 25,30 and 35 psi respectively. Line 1 is a linear fit for all of these data with $k=4.9$ and $\Delta \nu_{1}=573 \mathrm{~Hz}$ (see Eq.(3.56)). The arrows indicate the highest $\nu_{2}$ speeds before the motion of the inner-rotor crashes. The symbols $\circ, \bullet$ and $\diamond$ represent data for inner-rotor B (see text) for which $\bullet$ were obtained with an air pressure of $30 \mathrm{psi}$ and $\diamond$ with an air pressure of $35 \mathrm{psi}$; $\circ$ were obtained with variable air pressures. Line 2 is a linear fitting for $\bullet$ with $k=5.3$ and $\Delta \nu_{1}=1.4 \mathrm{kHz}$; line 3 is a linear fit for $\diamond$ with $k=5.4$ and $\Delta \nu_{1}=1.2 \mathrm{kHz}$. 
bearing and drive air pressures. The plot shows that the inner rotor speed depends linearly on the outer rotor speed over a wide range of $\nu_{1}$, but tends to deviate from it when $\nu_{1}$ comes closer to the point where the motion of the inner rotor crashes. The experimental slope $k$ obtained from the linear fitting is 4.9 for rotor $\mathrm{A}$ and is 5.4 for rotor B and does not depend on the air pressure used; moreover, these values are quite close to the calculated values. According to Eq.(3.56), this implies that the torque exerted on the inner rotor through the bearing adjusts itself with $\omega_{1}$ until it can no longer be tolerated by the bearing system. The intercept of $\nu_{2}$ at $\nu_{1}=0$ is not zero and is larger for rotor B which makes the deviation from the linearity more significant when $\Delta \nu_{2}=\Delta \omega_{2} / 2 \pi$ starts to change. The nonzero positive value of $\Delta \nu_{2}$ is in agreement with our argument discussed above based on the stability requirement that $\nu_{2}$ must be slightly exceed the value dictated by the torque-free condition. The relationship between $\Delta \nu_{2}$ and the air pressure used to operate the inner rotor depends on the details of the inner rotor bearing and drive system; as seen in Figure (3.24), the inner rotor $\mathrm{A}$ has a smaller $\Delta \nu_{2}$ value than $\mathrm{B}$ for the same air pressure and is less sensitive on the air pressure used.

Figure (3.25) shows ${ }^{23} \mathrm{Na}$ spectra of a sample containing a mixture of sodium sulfate and sodium oxalate of which the molar ratio of sodium atoms is 2:1. The static spectrum shown in Figure $(3.25 \mathrm{a})$ is $10 \mathrm{kHz}$ wide. Under MAS at $5.7 \mathrm{kHz}$ (Figure (3.25b)), the linewidth is reduced to $2.5 \mathrm{kHz}$ and some structure is visible. Under DOR (Figure (3.25c)), the line collapses into sharp lines and the two-peak structure is clearly revealed; the intensity ratio of the two components is $2: 1$ just as expected and the linewidth is $80 \mathrm{~Hz}(0.8 \mathrm{ppm})$ for both lines.

In the above sections, we have described two new methods, DAS and DOR. Both of them can effectively average out second order broadenings in the central transitions of spin half integer nuclei with large quadrupolar interactions; high resolution spectra can therefore be obtained with them. More applications to other spin half 

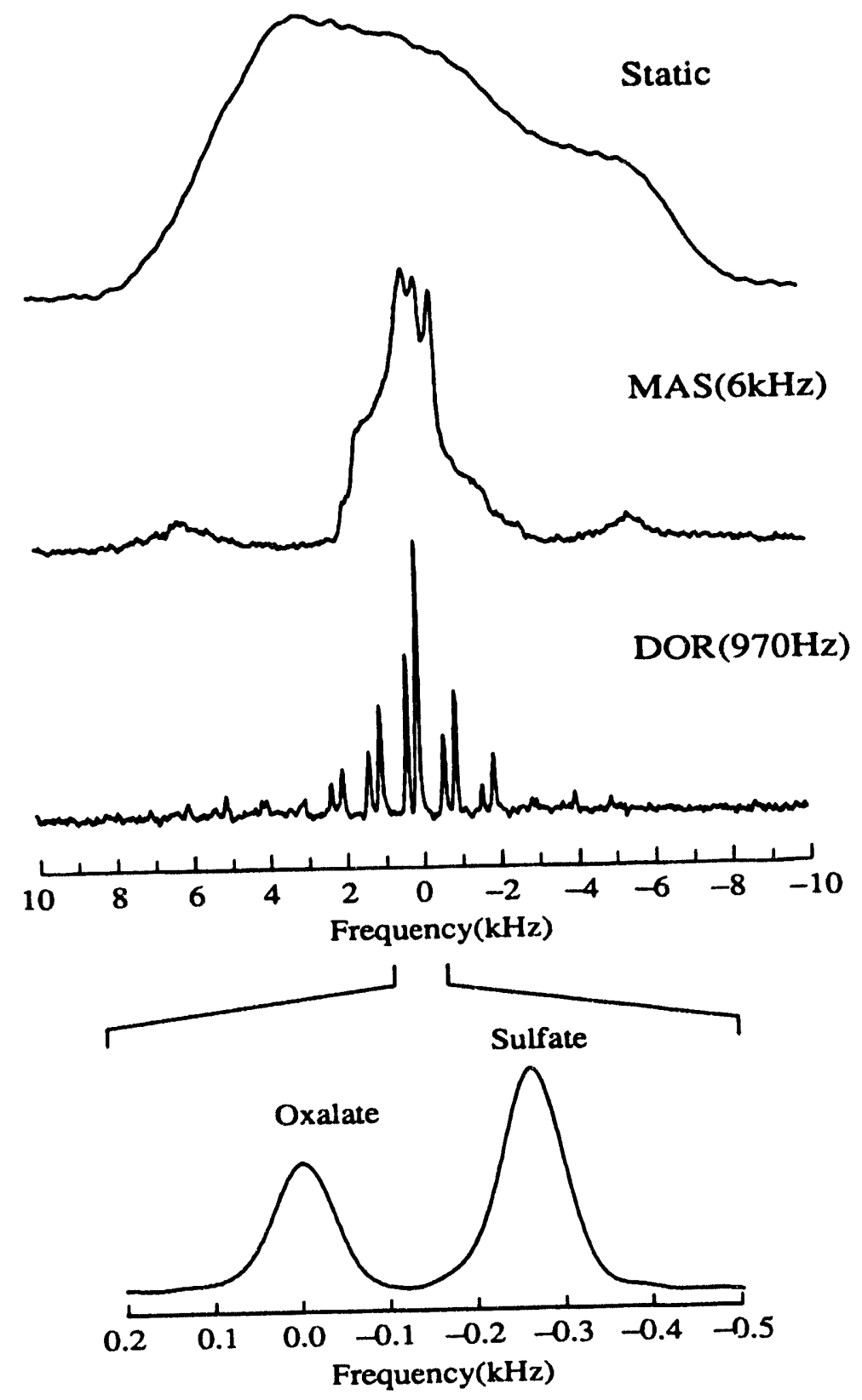

Figure 3.25: NMR spectra of ${ }^{23} \mathrm{Na}$ central transition in a 2:1 polycrystalline mixture of sodium sulfate and sodium oxalate. (a) is the static spectrum and (b) is the MAS spectrum with rotor spinning speed of $5.7 \mathrm{kHz}$. (c) is the DOR spectrum with $\nu_{2}=970 \mathrm{~Hz}$ clearly revealing the two line structure. (d) is a expandea version of (c) showing the centerband peaks originating from the two components of the mixture. 
integer nuclei, especially for ${ }^{17} \mathrm{O}$, can be found in reference [90]. We now turn to the description of the generalization of the DAS and DOR methods.

\subsection{Iterative Averaging of Tensorial Interactions}

In designing suitable averaging techniques, one should realize first that it is often unnecessary to implement full spherical symmetry. Since the anisotropy of the spin interactions is well defined, only a subset of the full rotation group is needed for efficient averaging. The problem then becomes one of determining which trajectories are both adequate and also feasible to implement through bulk macroscopic motions.

The orientational dependence of a nuclear or electronic dipolar transition frequency can be formally represented as a sum of components, each one irreducible under the rotation group $\mathrm{SO}(3)$ :

$$
\omega=\sum_{l} \omega_{l}
$$

The contribution of each component $\omega_{l}$ depends on the $2 l+1$ values of the corresponding tensor $A_{l, m}$ (see Eq.(3.20)), which forms the basis of the representation $D^{(l)}$ of $\mathrm{SO}(3)$. It also depends on the orientation $\Omega^{S F C}=(\alpha, \beta, \gamma)$ of the magnetic field in a sample-fixed coordinate (SFC) system (see Figure 3.3); namely from Eq.(3.25)

$$
\omega_{l}=\sum_{m} C_{l} A_{l, m} \mathcal{D}_{m, 0}^{(l)}\left(\Omega^{S F C}\right)
$$

where $\mathcal{D}_{m, 0}^{(l)}$ is an element of the associated Wigner rotation matrix (which is also a representation of the full rotation group) and $C_{l}$ is a constant given by Eq.(3.26). The particles in a heterogeneous sample are randomly oriented, and hence exhibit different sets of values $A_{l m}$,

$$
A_{l, m}=\sum_{m^{\prime}} \rho_{l, m^{\prime}} \mathcal{D}_{m^{\prime}, m}^{(l)}\left(\Omega^{P A S}\right)
$$

where the $\rho_{l, m^{\prime}}$ denote principal tensor components of rank $l$. For example, the principal components of a quadrupolar interaction tensor are given in Eq.(1.35) while 
the second order tensor of the quadrupolar interaction in the existing of a Zeeman interaction are given by Eq.(3.11). Dispersion in the Euler angles $\Omega^{P A S}$, reflecting different orientations of the sample-fixed coordinate frame relative to the local principal axis system (PAS), is the formal reason behind inhomogeneous line-broadening in NMR (or modulation of the intensity in optical spectroscopy or NQR). The isotropic part of the frequency in (3.57), $\omega_{0}$, is the same for each particle in the sample and thus is potentially measurable with the highest analytical resolution.

Spectroscopic measurement always requires a finite time to establish the differences between energy levels, during which the system continues to evolve and during which one can change the relative direction of the fields and of the sample. The orientation of the SFC, in general, is a function of time, and its motion imposes a time dependence upon the transition frequency. The observed phase of the signal, at some instant $T$, is proportional to

$$
\varphi_{l}(T)=\int_{0}^{T} d t \omega_{l}(t)=C_{l} \sum_{m} A_{l, m} \int_{0}^{T} d t \mathcal{D}_{m, 0}^{(l)}\left(\Omega^{S F C}(t)\right) .
$$

which reflects the cumulative effects of the changing resonant frequency. Stroboscopic measurement with a sampling period of $\mathrm{T}$, followed by a Fourier transformation, gives the average frequency of the transition over the interval. The simplest anisotropic term in the expansion of $\omega$, the dipolar term $\omega_{1}$, can be eliminated by summation over two opposite directions, $\Omega_{1}^{S F C}$ and $\Omega_{2}^{S F C}$, to give a net phase of zero at the sampling point. More generally, the anisotropy described by tensor components $A_{l m \neq 0}$ for a particular $l$ can be averaged away by directing the field at $N=l+1$ or more directions to form a cone (Figure 3.26 ), that is,

$$
\Omega_{k}^{S F C} \equiv\left(\alpha_{k}=\alpha_{0}+\frac{2 \pi}{N} k, \beta, \gamma\right)
$$

because

$$
\sum_{k=1}^{N} \mathcal{D}_{m, 0}^{(l)}\left(\Omega_{k}^{S F C}\right)=N d_{m, 0}^{(l)}(\beta) \delta_{m 0}
$$




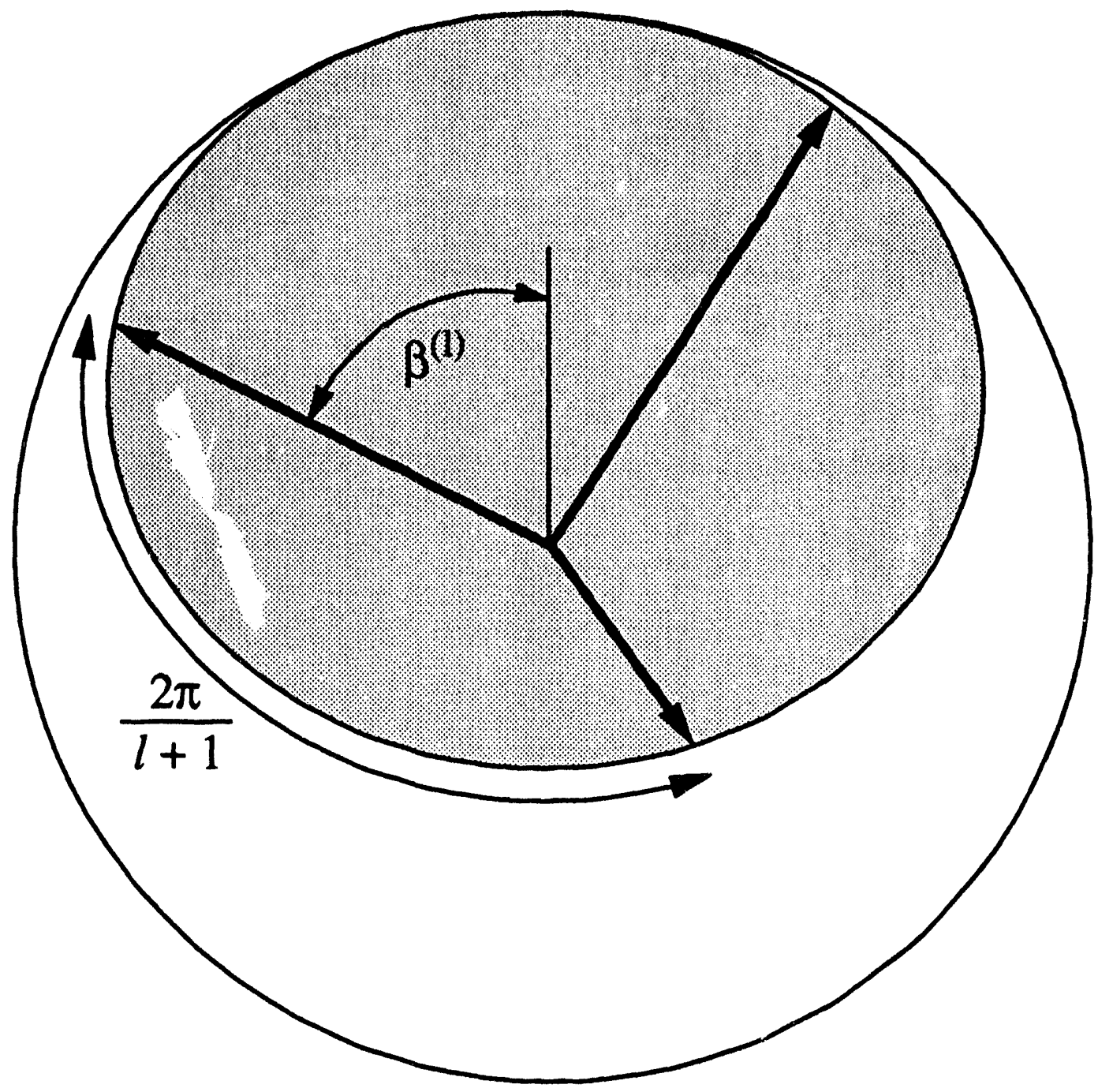

Figure 3.26: A basic succession of field directions that eliminates anisotropy due to a tensor of rank $l$. The magnetic field scans $l+1$ directions, forming a cone with opening $2 \beta^{(l)}$. 
in Eq.(3.60). The remaining term, proportional to $A_{t, 0}$, then can be eliminated by a suitable choice of the apex angle $\beta^{(l)}$

$$
d_{0,0}^{(l)}\left(\beta^{l}\right) \equiv P_{l}\left(\cos \beta^{(l)}\right)=0
$$

Values of $\beta^{(l)}$ for tensors up to rank $l=10$ are given in Table (3.7). In real space this Table 3.7: Roots of Legendre polynomials of $P_{l}\left(\cos \beta^{(l)}\right)$.

\begin{tabular}{|l|lllll|}
\hline \hline$l$ & \multicolumn{5}{|c|}{$\beta_{u}^{(l)}$} \\
\hline 1 & 90.00 & & & & \\
2 & 54.74 & & & & \\
3 & 39.23 & 90.00 & & & \\
4 & 30.56 & 70.12 & & & \\
5 & 25.02 & 57.42 & 90.00 & & \\
6 & 21.18 & 48.61 & 76.19 & & \\
7 & 18.36 & 42.14 & 66.06 & 90.00 & \\
8 & 16.20 & 37.19 & 58.30 & 79.43 & \\
9 & 14.50 & 33.28 & 52.17 & 71.08 & 90.00 \\
10 & 13.12 & 30.11 & 47.20 & 64.32 & 81.44 \\
\hline \hline
\end{tabular}

approach has been demonstrated in experiments involving the averaging of chemical shift anisotropy $l=2[87]$. In this work, the sample was mounted on a goniometer and reoriented so that the magnetic field assumed three orthogonal directions during the experiment. As the reorientation process was relatively slow, the magnetization was stored along the field direction during the reorientation. Magic angle spinning can be regarded as a continuous version of this (cubic) symmetry, in which the magnetic field traces out the continuous circle on the sphere, with the apex angle of the underlying cone determined by (3.63). This trajectory forms the basis of the MAS technique. 
Frequently, however, anisotropic interactions are determined by more than a single rank tensor, for example, the second order effect of the quadrupolar interaction in a magnetic field which is not strong enough to truncate the quadrupolar interaction. Under these condition the line broadening caused by combining several tensors simultaneously cannot be suppressed by rotation about a single axis, but rather only scaled and altered. For example, the lineshape of the central transition for half integer spin quadrupole nucleus is determined by the second- and fourth-rank tensors that describe the second order effect of the quadrupolar interaction. Figure (3.4) shows various calculated powder patterns of the central transition for spin $\left(I=\frac{3}{2}\right)$ in a sample spinning around a single axis at different angles. Figure (3.27) shows an experimental example of the central transition of $\mathrm{Na}-23$ nuclei in sodium oxalate at $105.8 \mathrm{M} \mathrm{Hz}$ under VASS. For standard MAS, the powder pattern is determined by the fourth rank tensor (Figure 3.27c). Spinning at either one of the magic angles of the fourth rank tensor results in spectra with lineshapes characteristic of the second rank tensor (Figure $3.27 \mathrm{~b}$ and $3.27 \mathrm{~d}$ ) while other possibilities give mixed results with some variation in linewidth (Figure 3.4)[103]. It is clear that the anisotropy due to more than one tensorial rank demands a better approximation to spherical symmetry. As an initial possibility we consider point subgroups $\Gamma$ of $\mathrm{SO}(3)$.

Owing to the high symmetries involved, the effect on the spectral components (3.57) can be conveniently analyzed with group theory. We are interested in the average value of each frequency component over all $N$ rotational operations $R$ of the symmetry group $G$ which obviously is a subgroup of the three dimensional rotation group $S O(3)$,

$$
{\overline{\omega_{l}}}^{C}=\sum_{m} A_{l, m} \overline{\mathcal{D}_{m, 0}^{(l)}}
$$

where

$$
\overline{\mathcal{D}_{m, 0}^{(l)}}=\frac{1}{N} \sum_{R \in G} \mathcal{D}_{m, 0}^{(l)}\left(\Omega_{R}\right)
$$



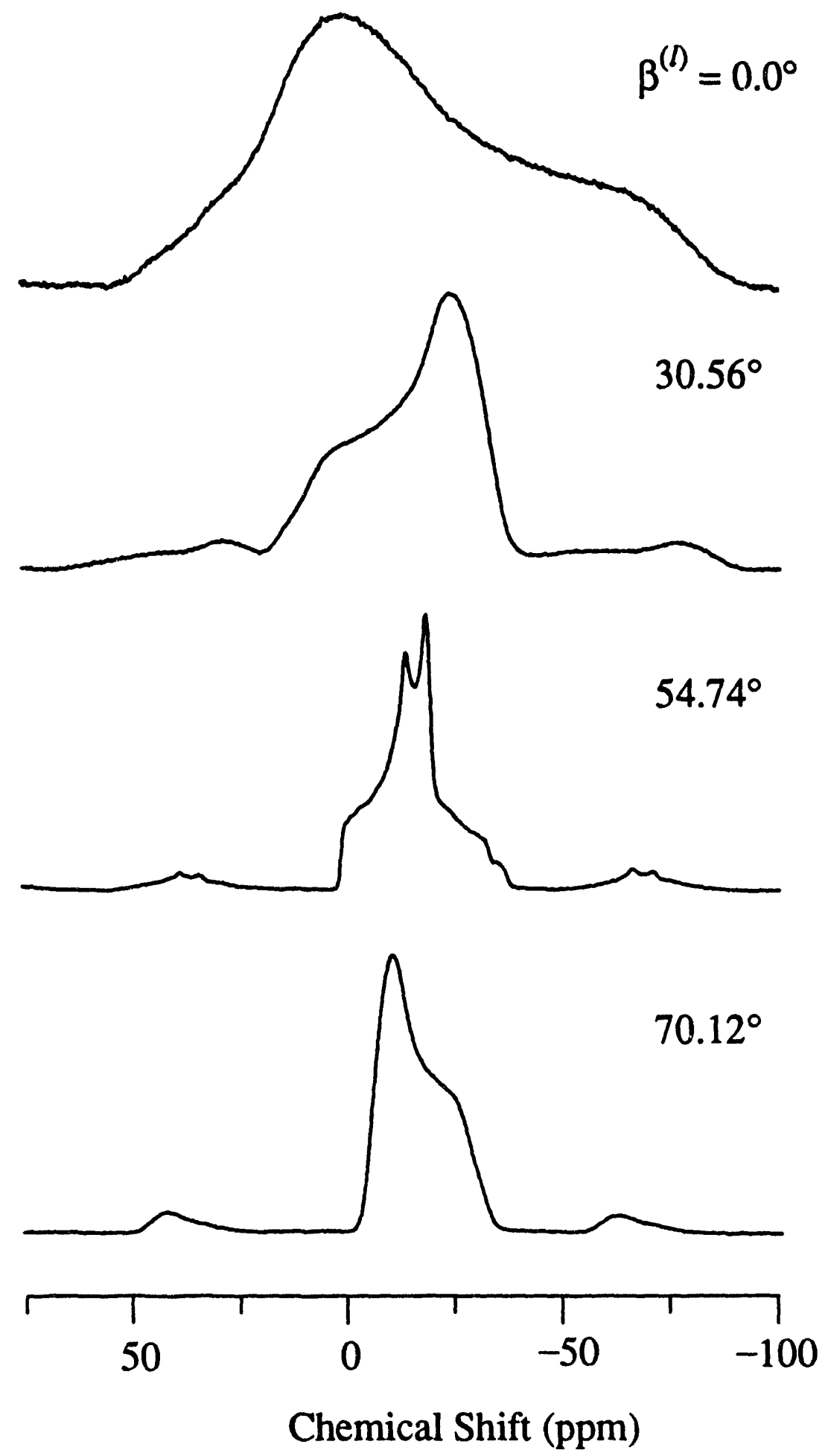

Figure 3.27: NMR spectrum of the central transition of $\mathrm{Na}-23$ in sodium oxalate at $108 \mathrm{MHz}$. a) Static sample, b) Sample spinning around axis at $30.56^{\circ}$, c) Sample spinning around axis at $54.74^{\circ}$, d) Sample spinning around axis at $70.12^{\circ}$. 
The representations of the group $\mathrm{SO}(3)$ reduce under subgroup $G$ as

$$
\mathcal{D}_{m^{\prime}, m}^{(l)}\left(\Omega_{R}\right)=\sum_{\Gamma} a^{(l \Gamma)} \mathcal{D}_{m^{\prime}, m}^{(l \Gamma)}\left(\Omega_{R}\right)
$$

The average of the transformation matrices (3.65) and also the averaged frequencies $\bar{\omega}_{l}^{\Gamma}$ can be different from zero only if expansion (3.66) contains the totally symmetric representation $A_{1}$. This follows directly from general orthogonality properties with respect to summation over group elements [68], namely from orthogonality with the symmetric identity representation, while

$$
\mathcal{D}_{m^{\prime}, m}^{\left(A_{1}\right)}(G)=\delta_{n^{\prime}, m}
$$

The multiplicity $a^{(l \Gamma)}$ of any irreducible representation $\Gamma$ can be evaluated from the general expression for traces

$$
a^{(l \Gamma)}=\frac{1}{N} \sum_{R} \chi^{(l)}(R) \chi^{(l \Gamma)}(R)
$$

and the characters calculated from

$$
\chi^{(l)}(R)=\sum_{m} \mathcal{D}_{m, m}^{(l)}\left(\Omega_{R}\right)=\frac{\sin \left(l+\frac{1}{2}\right) \zeta_{R}}{\sin \frac{1}{2} \zeta_{R}}
$$

where $\zeta_{R}$ is an angle of rotation of the corresponding symmetry operation, related to Euler angles by

$$
\zeta_{R}=2 \cos ^{-1}\left[\cos \frac{\beta_{R}}{2} \cos \frac{\left(\alpha_{R}+\gamma_{R}\right)}{2}\right]
$$

and the characters of the identity representation are

$$
\chi^{\left(l A_{1}\right)}(R) \equiv 1
$$

In Table (3.8) are shown characters of reducible $\mathcal{D}_{m^{\prime}, m}^{(l)}$ for all physically different symmetry operations (classes) of the most symmetrical, icosahedral finite rotation group $G=I$. In the last column we give the multiplicity of the symmetric representation $A_{1}$ after reduction of the original representation $\mathcal{D}_{m^{\prime}, m}^{(l)}$. Of the first ten anisotropic spectral components, only two $(l=6$ and $l=10)$ survive averaging under 
Table 3.8: Characters of $D_{m n}^{(l)}$ representations of the group $S O(3)$ after decomposition by the icosahedral group.

\begin{tabular}{|c|ccccc|c|}
\hline \hline $\mathrm{R}$ & $\mathrm{E}$ & $12 C_{5}$ & $12 C_{5}^{2}$ & $20 C_{3}$ & $15 C_{2}$ & \\
$\zeta_{R}$ & 0 & 72 & 144 & 120 & 180 & \\
\hline$l$ & & & & & & $a^{l A_{1}}$ \\
\cline { 1 - 3 } 0 & 1 & 1 & 1 & 1 & 1 & 1 \\
1 & 3 & $r$ & $\bar{r}$ & 0 & -1 & 0 \\
2 & 5 & 0 & 0 & -1 & 1 & 0 \\
3 & 7 & $-r$ & $-\bar{r}$ & 1 & -1 & 0 \\
4 & 9 & -1 & -1 & 0 & 1 & 0 \\
5 & 11 & 1 & 1 & -1 & -1 & 0 \\
6 & 13 & $r$ & $\bar{r}$ & 1 & 1 & 1 \\
7 & 15 & 0 & 0 & 0 & -1 & 0 \\
8 & 17 & $-r$ & $-\bar{r}$ & -1 & 1 & 0 \\
9 & 19 & -1 & -1 & 1 & -1 & 0 \\
10 & 21 & 1 & 1 & 0 & 1 & 1 \\
\hline \hline
\end{tabular}

icosahedral symmetry. A summary of the averaging of the tensor components under subgroups of $S O(3)$ is shown in Figure (3.28). Although icosahedral symmetry is very powerful, it has not yet found any widespread use in NMR except as a special case of dynamic angle spinning (DAS), a point to which we have discussed in section 3.4 and will return later for more.

Selective averaging of interactions with different ranks can be reconstructed iteratively. Given a certain set of directions $\Omega_{k}^{\prime}$ of the magnetic field in the sample-fixed coordinate (SFC) system, an additional splitting of directions around the original 
Symmetry

T2 $\left\langle\mathrm{A}_{l \mathrm{~m}}>=0\right.$

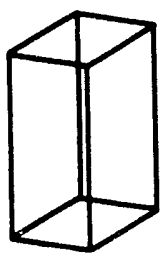

Tetragonal $\left(D_{4}\right)$

$$
l=\begin{array}{|l|l|l|l|l|l|l|l|l|l|l|}
\hline 0 & 1 & 2 & 3 & 4 & 5 & 6 & 7 & 8 & 9 & 10 \\
\hline
\end{array}
$$

Tetrahedral (T)

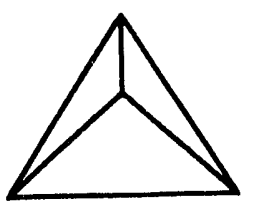

$$
l=\begin{array}{|l|l|l|l|l|l|l|l|l|l|l|}
\hline 0 & 1 & 2 & 3 & 4 & 5 & 6 & 7 & 8 & 9 & 10 \\
\hline
\end{array}
$$

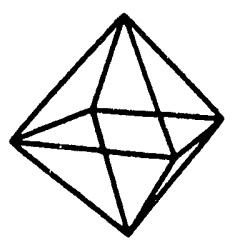

Octahedral (0)

$$
l=\begin{array}{|l|l|l|l|l|l|l|l|l|l|l|}
\hline 0 & 1 & 2 & 3 & 4 & 5 & 6 & 7 & 8 & 9 & 10 \\
\hline
\end{array}
$$

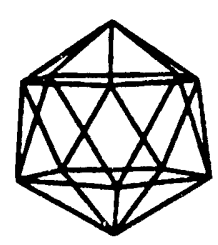

Icosahedral (I)

$$
l=\begin{array}{|l|l|l|l|l|l|l|l|l|l|l|}
\hline 0 & 1 & 2 & 3 & 4 & 5 & 6 & 7 & 8 & 9 & 10 \\
\hline
\end{array}
$$

\section{Rotation (SO(3))}

$$
l=\begin{array}{|l|l|l|l|l|l|l|l|l|l|l|}
\hline 0 & 1 & 2 & 3 & 4 & 5 & 6 & 7 & 8 & 9 & 10 \\
\hline
\end{array}
$$

Figure 3.28: Averaging of spherical harmonics under subgroups, (for our interest, symmetry point groups), $\left.D_{4}, T, O, I\right)$ of $\mathrm{SO}(3)$. 
ones, as shown in Figure (3.29) will give a stroboscopic phase of

$$
\varphi_{l}(T)=\frac{T}{N^{\prime} N^{\prime \prime}} \sum_{m^{\prime}, m} A_{l, m^{\prime}} \sum_{k}^{N^{\prime}} \mathcal{D}_{m^{\prime}, m}^{(l)}\left(\Omega_{k}^{\prime}\right) \sum_{j}^{N^{\prime \prime}} \mathcal{D}_{m, 0}^{(l)}\left(\Omega_{j}^{\prime \prime}\right),
$$

After averaging over the first set, $\Omega_{k}^{\prime}$, this can be expanded as

$$
\begin{aligned}
\varphi_{l}(T)= & \frac{T}{N^{\prime \prime}} \sum_{m=-l}^{l}\left(A_{l, 0} d_{0, m}^{(l)}\left(\beta^{\prime}\right) d_{m, 0}^{(l)}\left(\beta^{\prime \prime}\right) \sum_{j}^{N^{\prime \prime}} e^{-\imath m\left(\gamma^{\prime}+\alpha_{j}^{\prime \prime}\right)}\right. \\
& +\frac{1}{N^{\prime}} \sum_{m^{\prime}=N^{\prime}}^{l} A_{l, m^{\prime}} \sum_{k}^{N^{\prime}} \mathcal{D}_{m^{\prime}, m}^{(l)}\left(\Omega_{k}^{\prime}\right) \sum_{j}^{N^{\prime \prime}} \mathcal{D}_{m, 0}^{(l)}\left(\Omega_{j}^{\prime \prime}\right) \\
& \left.+\frac{1}{N^{\prime}} \sum_{m^{\prime}=-l}^{-N^{\prime}} A_{l, m^{\prime}} \sum_{k}^{N^{\prime}} \mathcal{D}_{m^{\prime}, m}^{(l)}\left(\Omega_{k}^{\prime}\right) \sum_{j}^{N^{\prime \prime}} \mathcal{D}_{m, 0}^{(l)}\left(\Omega_{j}^{\prime \prime}\right)\right)
\end{aligned}
$$

where the last two sums disappear if the multiplicity of the first splitting is $N^{\prime}>l$.

A tensor of any rank $l$ thus can be averaged if either $\beta^{\prime}$ or $\beta^{\prime \prime}=\beta^{(l)}$, and if the multiplicity of both splittings exceeds the rank, $N^{\prime},{ }^{\prime \prime}>l$. Two possibilities for selecting $\beta$ allow for simultaneous averaging of two tensors of different ranks. As can be seen from (3.73), the multiplicity of the first splitting can be reduced to $l_{1}+1$ if $l_{1}$ is the lowest of the two ranks. In that case the ordering of the apex angles also becomes important: $\beta^{\prime \prime}=\beta^{\left(l_{2}\right)}$, otherwise terms $A_{l, m^{\prime}} \mathcal{D}_{m^{\prime}, 0}^{\left(l_{2}\right)}\left(\beta^{\left(l_{2}\right)}\right) \mathcal{D}_{0,0}^{\left(l_{2}\right)}\left(\beta^{\left(l_{1}\right)}\right)$, where $m^{\prime}=-l_{2},-l_{2}+1, . .,-N^{\prime}, N^{\prime}, . ., l_{2}$, will not be averaged. Extension of this iterative procedure to further terms of different rank is obvious.

Straightforward extension of the multiple splitting to a continuous trajectory, involving multiple rotations, would also require multiple time dimensions. The ambiguity of the phase parameters $\gamma^{\prime}$ in (3.72), however offers possibilities for a onedimensional trajectory. Introducing a time dependence

$$
\begin{aligned}
\gamma_{i}^{\prime}+\alpha_{j}^{\prime \prime} & =\gamma_{0}+\omega_{r} t \text { and } \\
\alpha_{i}^{\prime} & =N \omega_{r} t,
\end{aligned}
$$

we can replace (3.72) by its continuous counterpart

$$
\varphi_{l_{1}, l_{2}}(T)=\sum_{l}^{l_{1}, l_{2}} \sum_{m^{\prime}, m} A_{l, m^{\prime}} d_{m^{\prime}, m}^{(l)}\left(\beta^{\left(l_{1}\right)}\right) d_{m, 0}^{(l)}\left(\beta^{\left(l_{2}\right)}\right) \int_{0}^{T} e^{-\imath \omega_{r} t\left(m^{\prime} N+m\right)+i n \gamma_{0}} d t .
$$




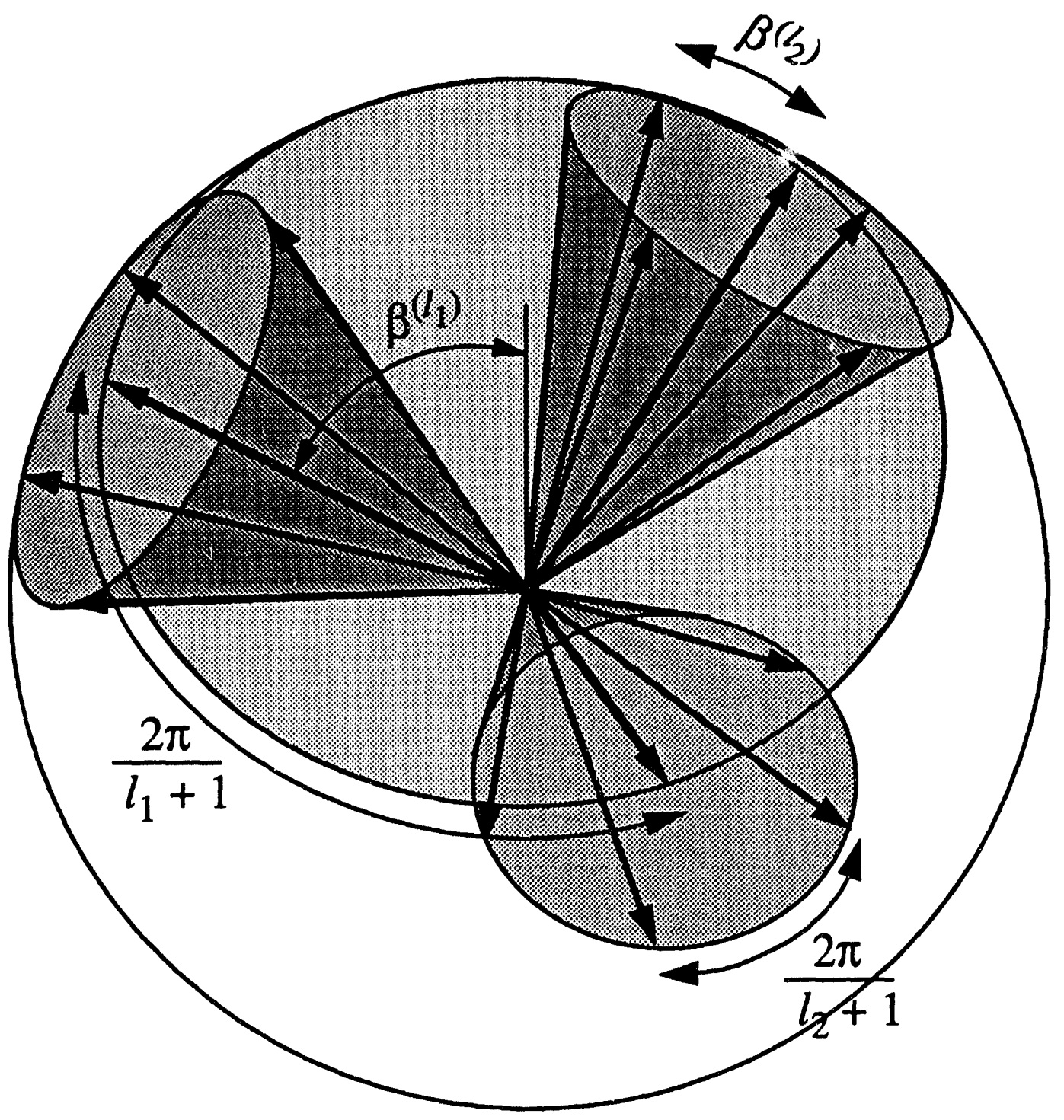

Figure 3.29: Tensor interactions of two ranks, $l_{1}$ and $l_{2}$, can be eliminated with an additional, or secondary splitting, of the field directions under which the interactions are averaged. The symmetry axes of the new cones retain the original symmetry of the primary splitting. 
If

$$
\omega_{r} T\left(m^{\prime} N+m\right)=k 2 \pi, \quad k=1,2, \ldots
$$

then expression (3.75) reduces to

$$
\varphi_{l_{1}, l_{2}}(T)=T \sum_{l} A_{l, 0} d_{0,0}^{(l)}\left(\beta^{\left(l_{1}\right)}\right) d_{0,0}^{(l)}\left(\beta^{\left(l_{2}\right)}\right)=0
$$

which describes a rotation of the field direction with a slow change in the rotation axis (Figure 3.30). Vertices of polygons with opening $2 \beta^{\left(l_{2}\right)}$ exactly fit this trajectory, while the symmetry axes of the polygons trace out a cone with opening angle $2 \beta^{\left(\ell_{1}\right)}$.

The iterative geometry described above was first applied to the averaging of second and third rank interactions in spin space, using double modulation of the $\mathrm{CW}$ irradiation [71]. In real space, applications based on an iterative scheme were used to average second order quadrupole line-broadening $[72,104]$. The fast reorientation is performed with a double rotor assembly (DOR), in which a powder sample is placed in a small rotor (inner rotor), spinning inside a larger rotor (outer rotor) (see Figure 3.23)[79]. The spinning axis is inclined at the angle $\beta^{\left(l_{4}\right)}$ relative to the symmetry axis of the outer rotor, which is itself spinning at $\beta^{\left(l_{2}\right)}$ relative to the magnetic field direction .

The third (and probably most general) averaging scheme, dubbed dynamic angle spinning (DAS) [76], is suggested by the patterns in Figure 3.27. Note that two of the lineshapes (Figure 3.27b an 3.27d) are in fact mirror images with different scaling factors. The reflection symmetry about an isotropic shift value is caused by a sign inversion of $P_{2}\left(\cos \beta^{(4)}\right)$, and the scaling ratio is given by

$$
\left|\frac{P_{2}\left(\cos \beta_{1}^{(4)}\right)}{P_{2}\left(\cos \beta_{2}^{(4)}\right.}\right|=1.87
$$

where $\beta_{1}^{(4)}=30.56^{\circ}$ and $\beta_{2}^{(4)}=70.12^{\circ}$, respectively. Since the shift reflection occurs with the same scaling ratio for each particle in the sample, it is possible to rephase the signal of all particles simultaneously at some time $T$. If evolution for a period 


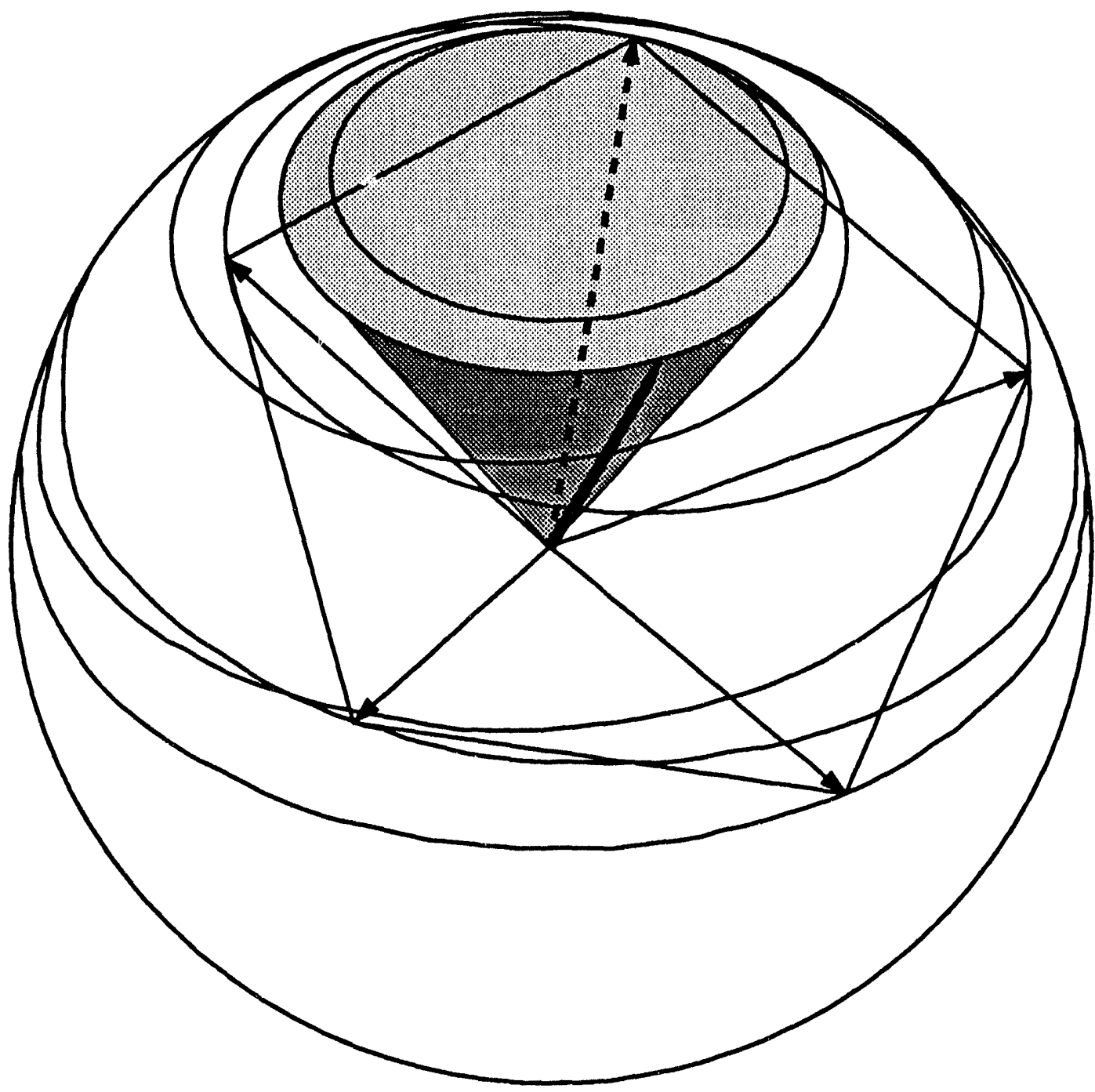

Figure 3.30: A continuous one-dimensional trajectory to eliminate two tensors. A complementary set of points (commensurate with the basic splitting) can be found at every point on the trajectory, to average one of the tensors. Such a set is shown here as a pentagon. The centers of the pentagons form a continuous cone with apex angle such that another tensor will be averaged. 
$\tau T$ proceeds at the angle $\beta_{1}$, and subsequently for a period $(1-\tau) T$ at $\beta_{2}$, the accumulated anisotropic phase will be

$$
\phi(T)=\int_{0}^{T} \omega(\beta(t)) d t=\left(\omega\left(\beta_{1}\right) \tau+\omega\left(\beta_{2}\right)(1-\tau)\right) T .
$$

The modulation (3.79) disappears for each interaction of rank $n$ if

$$
P_{n}\left(\cos \beta_{1}\right) \tau+P_{n}\left(\cos \beta_{2}\right)(1-\tau)=0
$$

which results in $\phi(T)=0$. A signal recorded at constant intervals of time $T, 2 T, 3 T, . .$, then will be independent of all anisotropic terms, and carry information only about the available isotropic shifts.

Many variations of this approach are possible because motion of the spinning axis is not limited to discrete positions and equal probability. Any function of time $\beta(t)$, satisfying the condition

$$
\int_{0}^{T} \omega(\beta(t)) p(t) d t=0
$$

where $p(t)$ is a weight function, will periodically refocus the phase. For example, linear sweep of the spinning axis between the angles $19.05^{\circ}$ and $99.19^{\circ}$ is a solution to (3.81). Similarly, the above DOR trajectory can be understood as

$$
\cos [\beta(t)]=\cos \left(\beta^{(2)}\right) \cos \left(\beta^{(4)}\right)+\sin \left(\beta^{(2)}\right) \sin \left(\beta^{(4)}\right) \cos \left(\omega_{r} t\right)
$$

and $p(t)=1$, where $\omega_{r}$ is the spinning speed of the outer spinner, and $\beta^{(2)}$ and $\beta^{(4)}$ are the magic angles of the Legendre polynomials $P_{2}(\cos \beta)$ and $P_{4}(\cos \beta)$. The DAS trajectories can also follow the symmetry of regular polyhedra. The solutions of (3.80) generally describe surfaces in three dimensions space $\left(\beta_{1}, \beta_{2}, \tau\right)$, as shown in Figure (3.31). Since the spinning angles can be interchanged, the surfaces exhibit inversion symmetry. A simultaneous solution for two equations of the form (3.80) is obtained at any point on a crossing line of two surfaces (Figure 3.31). Two solutions, $\left(37.38^{\circ}, 79.19^{\circ}, 1 / 2\right)$ and $\left(0^{\circ}, 63.43^{\circ}, 1 / 6\right)$, describe circles which traverse the vertices of an icosahedron (Figure 3.32) and thus present continuous extensions of icosahedral 

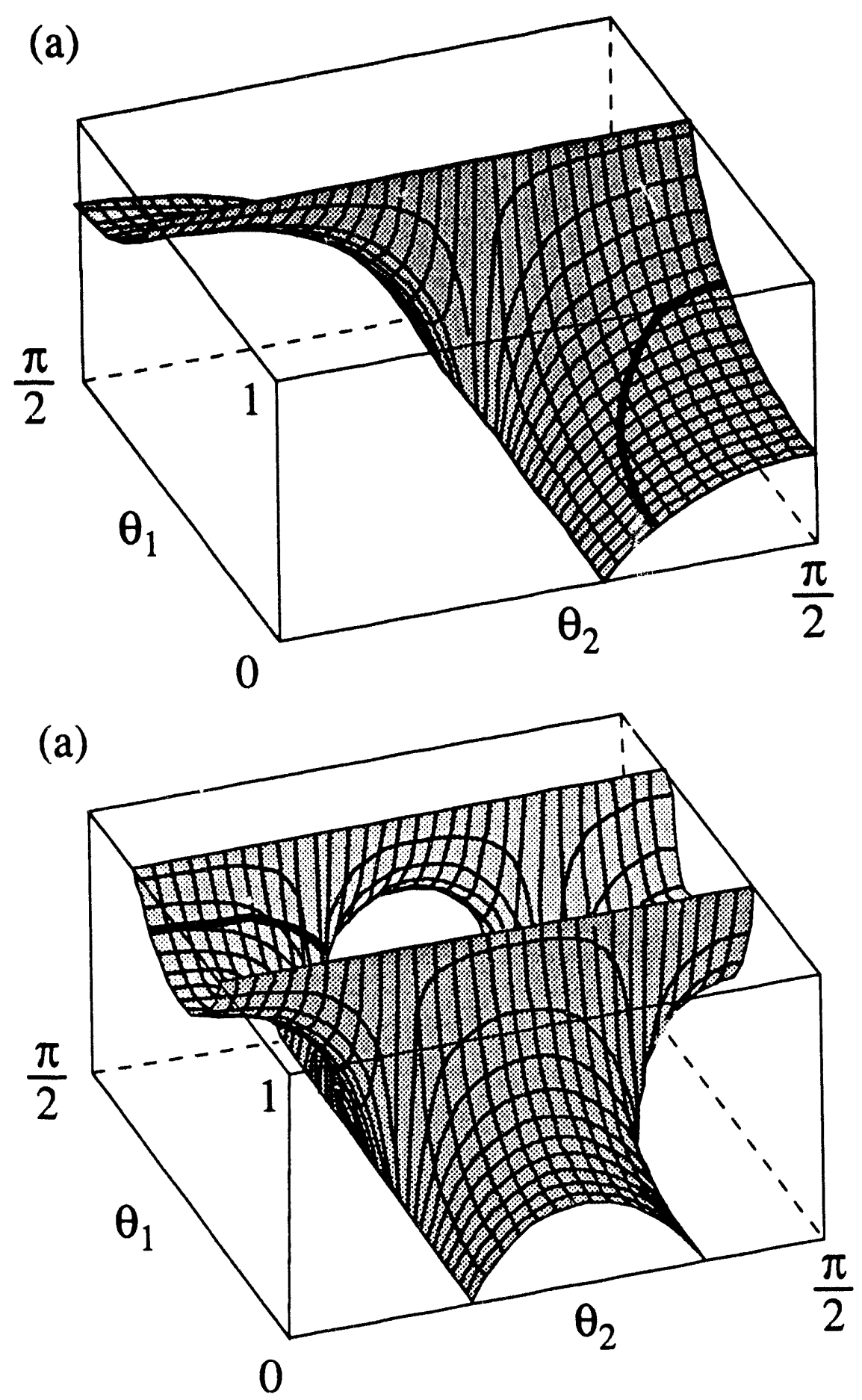

Figure 3.31: Solution of Eq.(3.59) for refocusing of tensor interactions of (a) second rank and (b) fourth rank, in a polycrystalline or amorphous sample. The line of intersection of the two surfaces, shown in bold, gives the simultaneous solution for anisotropies of both ranks. 

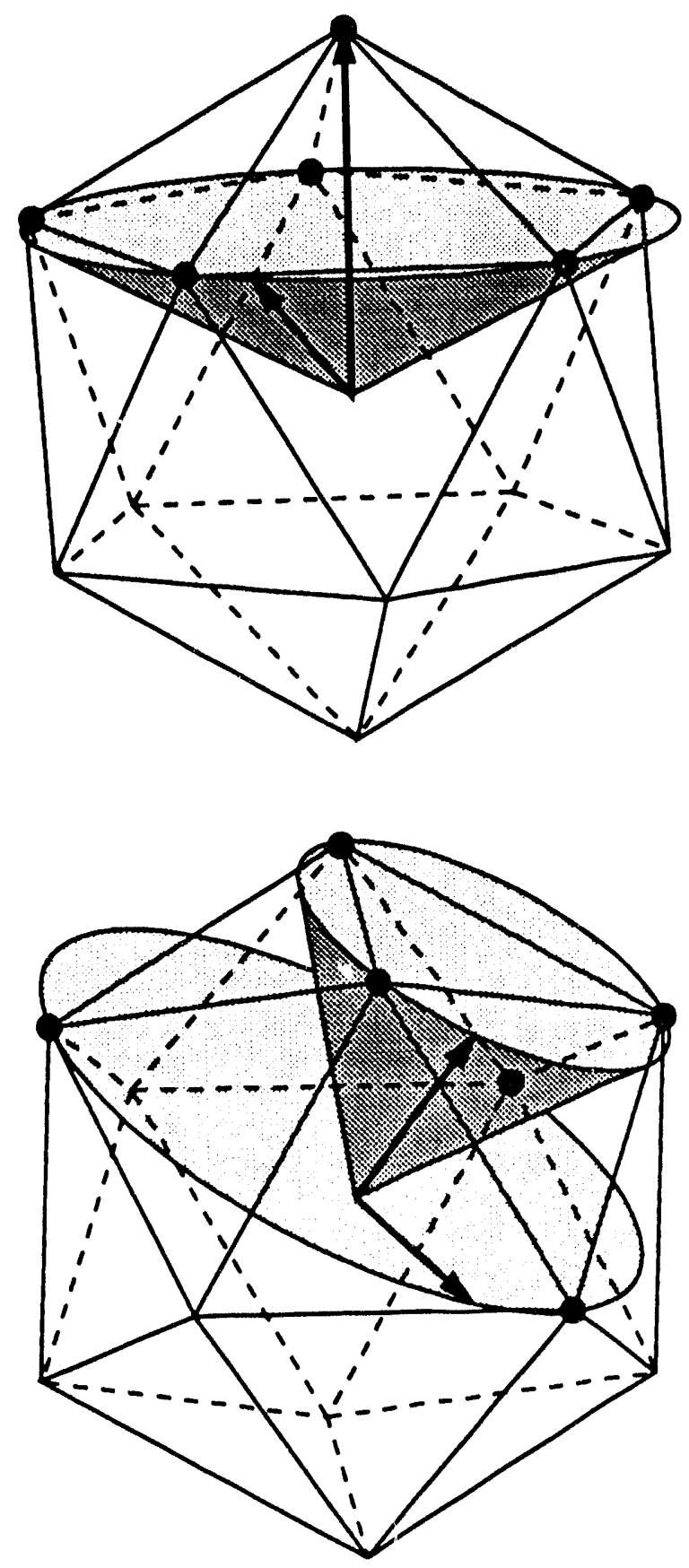

Figure 3.32: Icosahedral symmetry can be implemented with just two continuous trajectories in cases where tensors of rank two and four are to be eliminated. In the laboratory frame, this appears as a fast rotation of the sample at two different angles in succession. Time spent along one particular trajectory is proportional to the number of vertices. In top the vertices are those of an icosahedron, the two spinning axes are $\beta_{1}^{(l)}=0^{\circ}$ and $\beta_{2}^{(l)}=63.43^{\circ}$ and the ratio of times spinning at the two angles is 1:5. In bottom the vertices are those of either an icosahedron or a dodecahedron, the angles are $\beta_{1}^{(l)}=37.38^{\circ}$ and $\beta_{2}^{(l)}=79.12^{\circ}$ and the ratio of times spinning at the two angles is $1: 1$. 
point symmetry. One of the solutions may be converted to the other just by inversion of one direction. Equation (3.79) also can be extended to give solutions for averaging three or more tensors, with the addition of controlled time delays and spinning angles. Such experiments will be successful if the motion of the spinning axis is fast compared to the transverse relaxation rate, or if the transient magnetization can be stored along the polarizing field. The latter method was actually used in dynamic angle spinning (DAS) experiments to remove second order quadrupole line broadening in spectra of $0-17[78]$. We close by mentioning that averaging by icosahedral symmetry (or double rotation) in both spin and spatial coordinates simultaneously is an optimal solution for zero-field NMR in high field (see chapter 3)[50]. 


\section{Chapter 4}

\section{Calculation of Sideband Intensities in MAS, DAS, and DOR NMR}

\subsection{An Approximate Method of MAS Sideband Intensity Calculation}

\subsubsection{Introduction}

Magic angle spinning (MAS) in solid-state NMR as a powerful method for eliminating the dispersion in spectra caused by the orientation distribution of the resonance frequencies of the magnetization in polycrystalline or amorphous samples is obtaining more and more applications for studying molecular structure and dynamics. In contrast to the high resolution spectra in liquid NMR which results from the averaging under rapid incoherent tumbling of particles, the Zeeman transition frequencies of spin nuclei in the polycrystalline or amorphous samples are severely broadened owing to the lack of these random motions $[5,59]$. Such motions, where present, act to average anisotropic interactions (for example, chemical shift anisotropy(CSA), dipolar, and quadrupolar couplings between spin nuclei) to zero. Where the necessary averaging over the internal random motions of a spin system does not occur, macroscopic motions which are, in most of cases, coherent, have to be applied to the sample to improve spectral resolution. MAS, in which the sample rotates around an 
axis inclined at the magic angle $\left(\theta_{m}=54.74^{\circ}\right)$ with respect to the external static magnetic field $H_{0}$ is today one of the most common methods used for this purpose. This technique was first proposed by Andrew and Lowe in the late 1950s to suppress homogeneous broadening due to the dipolar interaction[56, 57, 58]. Later, Schaefer and Stejskal showed that CSA also can be averaged to zero at relative low fields [93]. In the extreme where the spinning speed far exceeds the breadth of the anisotropy, MAS yields the isotropic shift at the expense of any information concerning the anisotropy. This condition must be met for homogeneous broadening, for example, which is caused by homonuclear dipolar interactions. However, for inhomogeneous broadening (e.g. CSA and the first order quadrupolar interaction) sidebands develop around the isotropic peak if the spinning speed is smaller than the anisotropy[94]. Maricq and Waugh[95] subsequently proposed that the free induction decay (FID) signal be expanded as a series of moments in order to extract the anisotropic information from the sidebands. Herzfeld and Berger[96] also developed a general method, involving Bessel functions, to calculate sideband intensities. The anisotropic information is extracted by time-consuming simulations of the intensities of the individual sidebands. In this section we propose a new method to calculate approximate sideband intensities, in real time without using a large data base which is necessary in Herzfeld and Berger's method for time efficiency. The new method involves expanding the FID signal in a basis of irreducible spatial tensors in such a way, when averaged over all orientations, only zero rank irreducible tensors (scalar operators) contribute to the sideband intensities. Symmetry properties of the sidebands can be seen clearly in this expansion, and an approximate formula up to ninth-rank irreducible tensors is obtained by truncating the series. The dependence of sideband intensities on anisotropic parameters $(\delta, \eta)$ can then be expressed explicitly. With least square fitting programs, the extraction of the principal values of the chemical shift anisotropy from the sideband intensities obtained from MAS spectra can be 
quickly and easily performed.

\subsubsection{Theory}

We start with a rare spin nuclear system (such as ${ }^{13} \mathrm{C}$ ) in which nuclear spins interact with the external static magnetic field $H_{0}$ via anisotropic chemical shielding. According to the notation and conventions given by Mehring[19], we can represent the spin Hamiltonian as

$$
\mathcal{H}=\omega_{0} I_{z}+\omega_{0} \bar{\sigma} I_{z}+\omega_{0} \sum_{m=-2}^{2}(-1)^{m} A_{2-m} T_{2 m},
$$

where the $\omega_{0}$ is the Larmor frequency of the spin nuclear species involved, $\bar{\sigma}$ is the isotropic chemical shielding, $I_{z}$ is the spin operator, and $A_{2-m}$ and $T_{2 m}$ are the components of the second rank irreducible spatial and spin tensors respectively. In Eq.(4.1), the first term represents the Zeeman interaction, and the second term is the isotropic chemical shift while the third term the chemical shift anisotropy (CSA). The principal values, $\rho_{2 m}$, of the CSA tensor is given by

$$
\rho_{20}=\sqrt{\frac{3}{2}} \delta, \quad \rho_{ \pm 2}=\frac{1}{2} \eta \delta, \quad \rho_{ \pm 1}=0 .
$$

By using the properties of the irreducible tensors and the Wigner rotation matrices, the $A_{2, m}$ in Eq. (4.1), reflecting orientation dependence of the CSA Hamiltonian, can be expressed as

$$
A_{2 m}=\sum_{m=-2}^{2} D_{m m m}^{(2)}(\Omega) \rho_{2 m u}
$$

where $D_{m / m}^{(2)}(\Omega)$ are the Wigner rotation matrices and $\Omega=\Omega(\alpha, \beta, \gamma)$ are Euler angles.

After the spin Hamiltonian given in Eq.(4.1) is transformed into the rotating frame by the unitary operator $e^{-i \omega_{0} I_{z} t}$, the time dependent terms in the total Hamiltonian can be neglected under the first order perturbation approximation, and the total Hamiltonian becomes

$$
\mathcal{H}=\omega_{0} A_{20} \mathcal{T}_{20}
$$

assuming that the isotropic chemical shift $\left(\omega_{0} \bar{\sigma}\right)$ is zero in this particular case. 
After the sample rotates around a fixed axis inclined at an angle, $\theta$, with respect to the external magnetic field, viewed in the rotor frame fixed on the sample, the magnetic field, $H_{0}$, actually journeys on a cone with half apex angle $\theta$ (see Figure 3.13)). In order words, the magnetization traverses the spinning trajectory of the sample, and the local fields determined by the CSA tensor change periodically. Henceforth, the component of the second rank irreducible spatial tensor, $A_{20}$, becomes time dependent. By using the Wigner rotation matrices, the spin Hamiltonian in the rotating frame becomes

$$
\mathcal{H}=\gamma T_{20} \sum_{m=-2}^{2} D_{m, 0}^{(2)}\left(\Omega_{r}\right) A_{2 m},
$$

where $\Omega_{r}=\Omega_{r}\left(0, \theta, \omega_{r} t\right), \theta$ is the angle between the rotor axis and $H_{0}$, and $\omega_{r} t$ is the azimuth of the $x$-axis of the rotor frame with respect to $H_{0}$. In Eq. (4.5), the time-independent part, corresponding to $m=0$, disappears when $\theta=\theta_{m}$, the magic angle of the second order Legendre polynomial. The remaining components in Eq. (4.5) are time dependent. Since the sample spinning is only applied on the spatial parts of the spin Hamiltonian, the spin Hamiltonian always commutes with itself at all times. This means that the eigenvectors of the spin Hamiltonian remain unchanged at all times, but the eigenvalues are modulated by a set of harmonics. Hence the resonance frequency becomes time dependent, and the FID signal for a spin $I=\frac{1}{2}$ system can be written as

$$
g(t)=\exp \{-i \varphi(t)\}
$$

where

$$
\varphi(t)=\sqrt{\frac{2}{3}} \frac{\omega_{0}}{-i \omega_{r}} \sum_{m=-2}^{2} \frac{d_{m, 0}^{(2)}\left(\theta_{m}\right)}{m} A_{2, m} \phi_{m}
$$

and

$$
\phi_{m}=\left(\exp \left\{-i m \omega_{r} t\right\}-1\right) .
$$

In general, Eq. (4.6) describes a phase-modulated signal with the associated Fourier spectrum showing a band structure. Each oriented single crystal contributes 
a particular sideband pattern, and what we see is the average over all orientations for a powder sample. Such an averaged sideband pattern is not related simply to the anisotropies and asymmetry parameters of the CSA tensors, however. In order to extract these parameters from the experimental results obtained under MAS, Maricq and Waugh expanded Eq. (4.6) in a multiple moment series, and found that the second and third moments of the MAS NMR spectra are indeed related fairly simply to $\delta$ and $\eta$. In practical applications of the moment analysis method, the second and third moments are first calculated from the sideband intensities and the spinning speeds of the sample obtained from the experimental MAS NMR spectra, and then, using the relationships between the moments and the prir.cipal vilues of the CSA tensors, the anisotropies and asymmetry parameters can be calcuiated. Since the intensity of the $N$-th order sideband, in general, decays, but the frequency increases as the order of the sideband, $N$, increases, the contribution of small sideband intensity to the moments can not be ignored, and therefore, this method requires very accurate measurement of all sideband intensities, which is difficult to do. Moreover, the method fails when sidebands originating from different site in a spin system overlap. To overcome these problems, Herzfeld and Berger first expanded Eq. (4.6) using Bessel functions and subsequently converted it to a Fourier series. The $N$-th coefficient in the Fourier expansion then corresponds to the $N$-th sideband intensity. Nevertheless, the intensity of each sideband has a very complicated dependence on the anisotropic parameters, and the problem can be inverted only by time-consuming numerical simulations.

The dependence of the intensities on anisotropic parameters is complicated because the integrals over all orientations in Eq. (4.6) cannot be solved analytically. Here, instead, we will expand the FID signal into a Taylor series. By virtue of the properties of the products of two irreducible tensors, the FID signal is recast in a basis of irreducible tensors from rank zero to rank infinity. The rotational transfor- 
mation properties of irreducible tensors yield analytical solutions for the integrals up to any order.

The first step in this new method is to expand Eq. (4.6) into a Taylor series to obtain

$$
g(t)=\sum_{k} \frac{1}{k !}[\varphi(t)]^{k}
$$

Substituting Eq. (4.7) into (4.9), we then have

$$
g(t)=\sum_{k} \frac{1}{k !}\left(\sqrt{\frac{2}{3}} \frac{\omega_{0} \delta}{\omega_{r}}\right)^{k} f_{k}(t)
$$

where

$$
f_{k}(t)=\sum_{m_{1} \neq 0} \sum_{m_{2} \neq 0} \ldots \sum_{m_{k} \neq 0} B\left(m_{1}, m_{2}, \ldots, m_{k}\right) \phi_{m_{1}} \phi_{m_{2}} \ldots \phi_{m_{k}} A_{2, m_{1}} A_{2, m_{2}} \ldots A_{2, m_{k}}
$$

and

$$
B\left(m_{1}, m_{2}, \ldots, m_{k}\right)=\frac{d_{m_{1}, 0}^{(2)}\left(\theta_{m}\right) d_{m_{2}, 0}^{(2)}\left(\theta_{m}\right) \ldots d_{m_{k}, 0}^{(2)}\left(\theta_{m}\right)}{m_{1} m_{2} \ldots m_{k} \delta^{k}}
$$

Later, we will see that the function $f_{k}(t)$ only depends on the asymmetry parameter $\eta$.

In the next step, we introduce the product of two irreducible tensors given by[20]

$$
A_{l_{1}, m_{1}} A_{l_{2}, m_{2}}=\sum_{l=\left|l_{1}-l_{2}\right|}^{l_{1}+l_{2}} C\left(l_{1}, l_{2}, l ; m_{1}, m_{2}, m_{1}+m_{2}\right) A_{l, m_{1}+m_{2}}
$$

where $C\left(l_{1}, l_{2}, l, m_{1}, m_{2}, m_{1}+m_{2}\right)$ are the Clebsch-Gordan coefficients. Iterating using Eq. (4.13), we can represent the product of $k$ second-rank irreducible tensors as

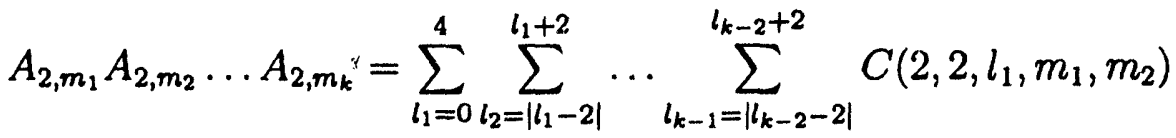

$$
\begin{aligned}
& C\left(l_{1}, 2, l_{2}, m_{1}+m_{2}, m_{3}\right) \ldots C\left(l_{k-2}, 2, l_{k-1}, \sum_{i=1}^{k-1} m_{i}, m_{k}\right) A_{l_{k-1}, \sum_{i=1}^{k} m_{i}} .
\end{aligned}
$$

With the orientation dependence expressed in terms of the Wigner rotation matrices, the average of the $l$-th rank irreducible spatial tensor over all orientations is

$$
\overline{A_{l, m}}=\left\{\begin{array}{lc}
A_{0,0}\left(l_{1}, \ldots, l_{k-3}\right) & \text { if } l=0 \text { and } m=0 \\
0 & \text { otherwise }
\end{array}\right.
$$


Hence after the powder average, only the scalar part in Eq. (4.11) remains:

$$
\begin{gathered}
A_{0,0}\left(l_{1}, \ldots, l_{k-3}\right)=\sum_{n_{1}, \ldots, n_{k-1}=-2}^{2} C\left(2,2, l_{1}, n_{1}, n_{2}\right) C\left(l_{1}, 2, l_{2}, n_{1}+n_{2}\right) \ldots \\
C\left(2,2,0, \sum_{i=1}^{k-1} n_{i},-\sum_{i=1}^{k-1} n_{i}\right) \rho_{2, n_{1}} \rho_{2, n_{2}} \ldots \rho_{2, n_{k-1}} \rho_{2,-} \sum_{i=1}^{k-1} n_{i} .
\end{gathered}
$$

In this equation, the product of $k$ components $\rho_{2, n_{i}}(i=1 \ldots k)$ is of order $\delta^{k}$, and will cancel with the $\delta^{k}$ in coefficient $B$ of Eq. (4.11). Thus $f_{k}(t)$ is only a function of the asymmetry factor $\eta$. From Eq. (4.2), the power of the asymmetry factor in $f_{k}(t)$ is determined by

$$
n=\left[\frac{\left|n_{1}\right|}{2}\right]+\left[\frac{\left|n_{2}\right|}{2}\right]+\ldots+\left[\frac{\left|n_{k}\right|}{2}\right] .
$$

The result of the square-brackets, representing the truncation of each individual term in Eq. (4.17), is an integer with the value zero or one. Since from Eq.(4.3), $\rho_{n_{i}}$ are not equal zero only if $n_{i}=0, \pm 2$, and since the sum over all indices, $\sum_{i=1}^{k} n_{i}$, has to be zero after the powder average according to Eq.(4.15), the number of indices which has value 2 must equal the number of indices with value -2 . Thus Eq. (4.17) can only result in an even integer number. This means that the power of $\eta$ must be even and, consequently, the sideband intensities are not sensitive to the sign of the asymmetry factor. This feature follows, then, that the sample rotation does not change the symmetry of the spin system, in agreement with the relationship between the static powder lineshapes and the asymmetry parameters $\eta$, (usually we can take $0 \leq \eta \leq 1$.).

The symmetry property of coefficients B can be easily found after the powder average by use of the properties of reduced Wigner rotation matrices:

$$
B\left(m_{1}, m_{2}, \ldots, m_{k}\right)=(-1)^{k} B\left(-m_{1},-m_{2}, \ldots,-m_{k}\right) .
$$

In the final step, from Eqs. (4.14) and (4.15) we have $m_{k}=\sum_{i=1}^{k-1} m_{i}$. Inserting this condition into Eq. (4.11), we thus obtain

$$
\phi_{m_{1}} \quad \ldots \phi_{m_{k-1}} \phi_{-\sum_{i=1}^{k-1} m_{i}}=\frac{1+(-1)^{k}}{2}+2 \sum_{n=1}^{k}(-1)^{n} \sum_{j_{1}=1}^{k} \ldots \sum_{j_{n}=j_{n-1}+1}^{k}
$$




$$
\begin{cases}\cos \left[\left(m_{j_{1}}+\ldots+m_{j_{n}}\right) \omega_{r} t\right] & \text { for even } k \\ i \sin \left[\left(m_{j_{1}}+\ldots+m_{j_{n}}\right) \omega_{r} t\right] & \text { for odd } k\end{cases}
$$

In Eq. (4.19), when $k$ is an even number, the product of $k$ functions $\phi_{i}(i=1 \ldots k)$ is also an even function about $N \omega_{r} t$; otherwise, it is an odd. This means that even order terms in the Taylor expansion symmetrically correct the intensities of sidebands whereas the odd terms result in the differences of sideband intensities about the centerband. Because the odd terms are antisymmetric, they make no contribution to the centerband.

With substitution of Eqs. (4.15),(4.16), and (4.19) into Eq.(4.11), $\overline{f_{k}(t)}$ can be expressed by

$$
\overline{f_{k}(t)}=\sum_{m=-2 N}^{2 N} \sum_{n=0}^{N} I_{k, m, n} \eta^{2 n} \exp \left(-i m \omega_{r} t\right)
$$

where $N=[k / 2]$ and

$$
\begin{aligned}
& I_{k, m, n}=\sum_{j=0}^{k}(-1)^{j} \sum_{l_{1}, \cdots, l_{k-3}} \sum_{m_{1}, \cdots, m_{k-1} \neq 0} \sum_{n_{1}, \cdots, n_{k-1}} B^{\prime}\left(m_{1}, \cdots, m_{k-1},-\sum_{i=1}^{k-1} m_{i}\right) \\
& \times C\left(2,2, l_{1} ; m_{1}, m_{2}\right) C\left(l_{1}, 2, l_{2} ; m_{1}+m_{2}, m_{3}\right) \cdots C\left(2,2,0, \sum_{i=1}^{k-1} m_{i},-\sum_{i=1}^{k-1} m_{i}\right) \\
& \times C\left(2,2, l_{1} ; n_{1}, n_{2}\right) C\left(l_{1}, 2, l_{2} ; n_{1}+n_{2}, n_{3}\right) \cdots C\left(2,2,0, \sum_{i=1}^{k-1} n_{i},-\sum_{i=1}^{k-1} n_{i}\right) \\
& \times \rho_{2, n_{1}}^{\prime} \cdots \rho_{2,-\sum_{i=1}^{k-1} n_{i}}\left[\delta\left(m-\sum_{i=1}^{j} m_{i}\right)+(-1)^{k} \delta\left(m+\sum_{i=1}^{j} m_{i}\right)\right] \delta\left(2 n-\sum_{i=1}^{k-1} \frac{\left|n_{i}\right|}{2}\right)
\end{aligned}
$$

and $B^{\prime}=\delta^{k} B$, and $\rho_{2 m}^{\prime}$ are equal to $\rho_{2, m}$ with $\delta=1$ and $\eta=1$ given in Eq.(4.3), that is, they are no longer functions of $\delta$ and $\eta$.

\subsubsection{Results}

In the last section, we have solved the powder average up to infinitive order in the Taylor expansion of the FID signal. After substitution of Eqs. (4.11), (4.16), and 
(4.17) into (4.9), however, the FID signa! can be represented as a Fourier series again (see Eq. (4.20)) and the sideband intensities can be obtained by evaluating the Fourier coefficients. As the order in the Taylor expansion increases, the number of summations over all Clebsch-Gordan coefficients also increases as $(k-3)$, where $k$ is the $k$-th order in the expansion. Using a computer, it is easy to determine the coefficients of the first ten orders in the Taylor expansion, but calculation of higherorder coefficients becomes very time consuming. Fortunately, though, in practice the spinning speed is not much smaller than the CSA (especially for ${ }^{13} \mathrm{C}$ ), and in these circumstances the approximation up to ninth order, as we will see, is already very good for the calculation of sideband intensities.

Here we only list the coefficients of the first four orders in the Taylor expansion and use them to draw some general properties of the sideband intensities under MAS. All other coefficients can be obtained from Eqs. (4.11), (4.14), (4.16), and (4.19). Thus,

$$
\begin{aligned}
& \overline{f_{0}(t)}=1 \\
& \overline{f_{1}(t)}=0 \\
& \overline{f_{2}(t)}=\frac{1}{5}\left(3+\eta^{2}\right)\left[-\frac{3}{4}+\frac{2}{3} \cos \left(\omega_{r} t\right)+\frac{1}{12} \cos \left(2 \omega_{r} t\right)\right] \\
& \overline{f_{3}(t)}=\frac{-3 i}{35} \sqrt{\frac{3}{2}}\left(-1+\eta^{2}\right)\left[2 \sin \left(\omega_{r} t\right)-\sin \left(2 \omega_{r} t\right)\right]
\end{aligned}
$$

First, it can be seen from Eq. (4.22) that the zero order term is always equal one while the first order term is zero. This implies that the first order term has no correction to the first order $( \pm 1)$ sidebands due to the asymmetry parameter. This explains that once the spinning speed is in the regime of the linewidth of the static powder pattern the sideband intensities measured experimentally become more or less symmetric around the centerband. Such a distribution of the sideband intensities is no longer sensitive to the asymmetry parameter and therefore cannot be used to extract the anisotropic information. Second, when $\eta=1$, the value of $\overline{f_{3}(t)}$ is zero. 

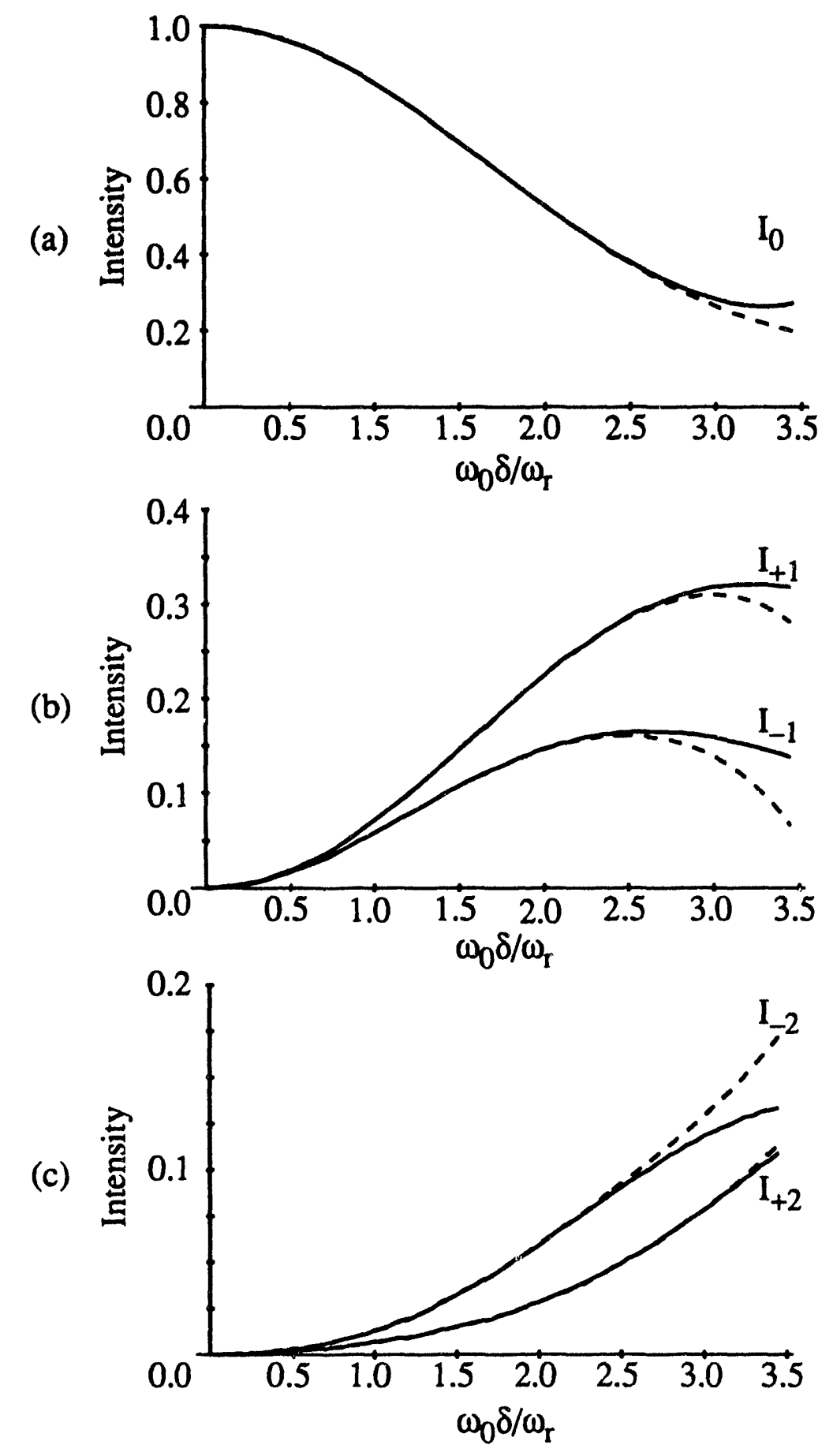

Figure 4.1: Variation of sideband intensities with the ratio of chemical shift anisotropy $\left(\omega_{0} \delta\right)$ to spinning speed $\left(\omega_{r}\right)$, computed for the case $\eta=0.5$. Solid lines are calculated by numerically integrating Eq. (6) over oll orientations, and dashed lines are obtained by our approximate method (up to the ninth order). (a) Centerband intensities. (b) First-order sidebands $( \pm 1)$. (c) Second-order sidebands $( \pm 2)$. 
This conclusion can be extended to all odd terms in accordance with the symmetry properties of odd and even orders in the Taylor expansion as discussed at the end of the last section. Intuitively, the powder pattern is symmetric around the isotropic frequency once $\eta=1$. Thus the sidebands should also be symmetric around the centerband. Third, the sum of all coefficients of sidebands in $\overline{f_{k}(t)}$ is always zero except for $k=0$. This means that the correction of each order just redistributes each sideband intensity over the whole set of sidebands, and the FID signal is always normalized.

In order to see how good the approximate method is, we have to evaluate the sideband intensities exactly from Eq. (4.6). According to the result given by Herzfeld and Berger[96], the intensity of the $N$ th sideband is

$$
I_{N}=\frac{1}{16 \pi^{4}} \int_{0}^{\pi} \int_{0}^{2 \pi} \sin (\beta) d \beta d \gamma\left|\int_{0}^{2 \pi} \exp -i[N \theta+\psi(\theta)] d \theta\right|^{2}
$$

where

$$
\psi(\theta)=\sqrt{\frac{2}{3}} \frac{\omega_{0}}{-i \omega_{r}} \sum_{m=-2}^{2} \frac{d_{m, 0}^{(2)}\left(\theta_{m}\right)}{m} A_{2, m} \exp (-i m \theta)
$$

Composite ten-point Gaussian (Gauss-Legendre) quadruture has been used to evaluate the three-dimensional integral in Eq. (4.24). To calculate the intensities of a set of sidebands between one half and five minutes on Micro VAX II depending on the accuracy needed. Figure (4.1) shows that a comparison of sideband intensities computed through the first ten orders of the Taylor expansion (dashed line) and the exact solution (solid line) obtained by numerical simulation[96]. Since the ratio of the number of multiplications involved in the numerical integration of the exact solution given in Eq. (4.23) to that in the approximate method is at least in the order of ten thousands, the computing time reduces from about six hours for the exact result to a few seconds for the approximate method. Both results are very close when the ratio of the anisotropy to the spinning speed, $\frac{\omega_{0} \delta}{\omega_{r}}$, is smaller than three. For $\frac{\omega_{0} \delta}{\omega_{r}}>3$, the sideband intensities calculated by the approximate method, however, tend to diverge. Figure (4.2) shows the convergence under approximations 
(a)

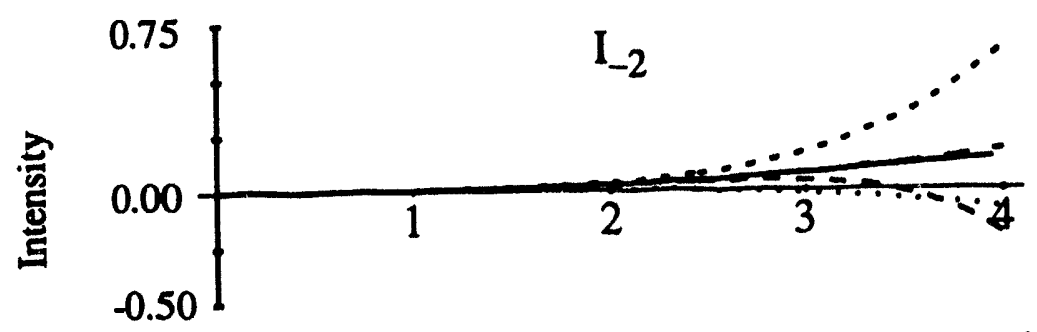

(b)

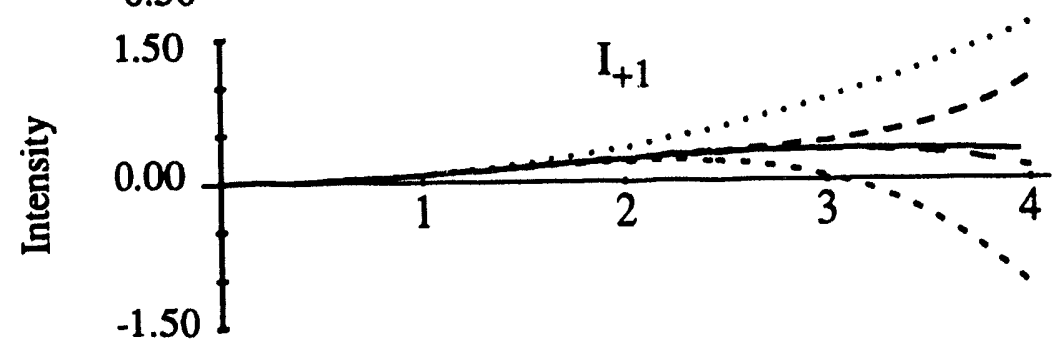

(c)

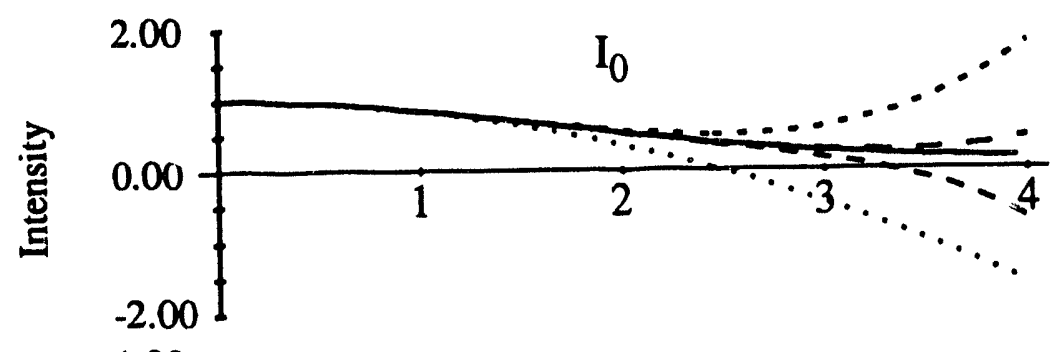

(d)

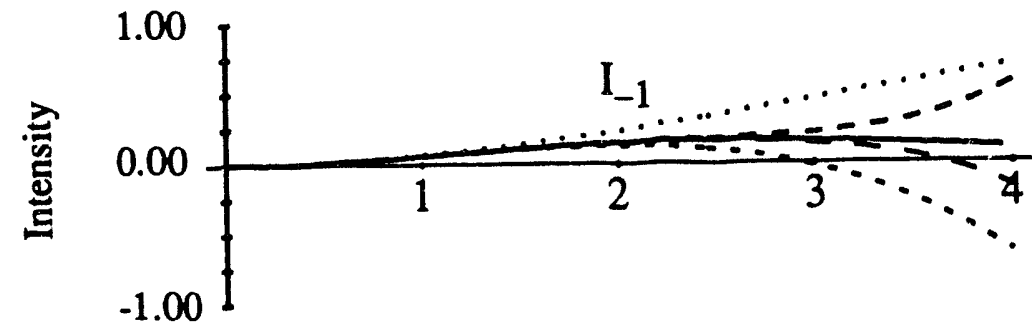

(e)

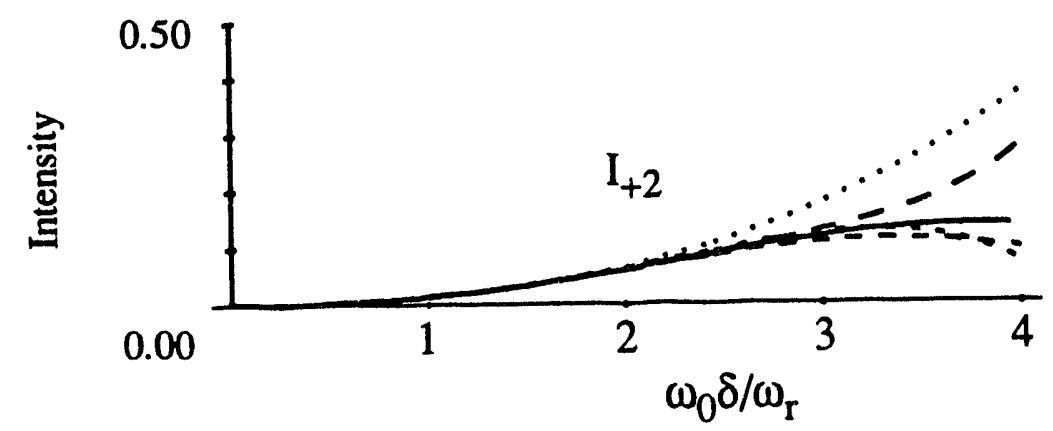

Figure 4.2: Variation of sideband intensities with the ratio of chemical shift anisotropy $\left(\omega_{0} \delta\right)$ to spinning speed $\left(\omega_{r}\right)$ under the approximate method, with first four (dotted lines), six (short dashed lines), eight (long dashed lines), and ten (solid lines) orders. $\eta=0.5$. (a,e) Second-order sideband. (b,d) First-order sideband. (c) centerband. 
of different order. We see that the sideband intensities converge at higher orders, and that the rate of convergence is much the same as for the sinusoidal functions. The total intensity obtained from the sum over all sideband intensities is always one, even though each sideband itself diverges. This is because the higher order sideband intensities are given by redistributing the lower order sideband intensities and keeping the whole intensity of the spectrum at unity. So, the divergence comes from the incorrect intensity partition of each sideband for large value of $\frac{\omega_{0} \delta}{\omega_{r}}$.

In order to extract anisotropic information, the experimental sideband intensities have to be normalized for comparison with the theoretical values. Experimental signal-to-noise therefore must be good enough to permit accurate measurement and summation of all sideband intensities. To overcome this requirement, Herzfeld and Berger proposed an alternative method in which the anisotropic information is extracted by measuring the ratios of the sideband intensities to the centerband intensity. Furthermore, as mentioned above, the differences of sideband intensities around the centerband are relatively sensitive to the asymmetry factor $(\eta)$, whereas the averages of these sideband intensities are sensitive only to the anisotropy $(\delta)$ (see Figure 4.3). Although there is a maximum difference of the $N$-th order sideband intensities around centerband, the change of the difference is the minimum at axial and near-axial situations. This method therefore is only slightly better than the method used by Herzfeld and Berger. We use the ratios of the differences (averages) to the centerband intensity to extract the anisotropic parameters by least squares fitting. In the fitting program (MASFIT), the initial values of the anisotropic parameters are calculated by Eq. (4.22), and then by use of the Davidon-Fletcher-Powell (DFP) algorithm[98], the anisotropic parameters can be extracted in a few seconds. Figure (4.4) shows contours of the surface used in the fitting program. We can clearly see that there does exist a unique minimum, but that the surface is very smooth in the dimension of the asymmetry factor $\eta$. As a result, the determination of $\eta$ is relatively 

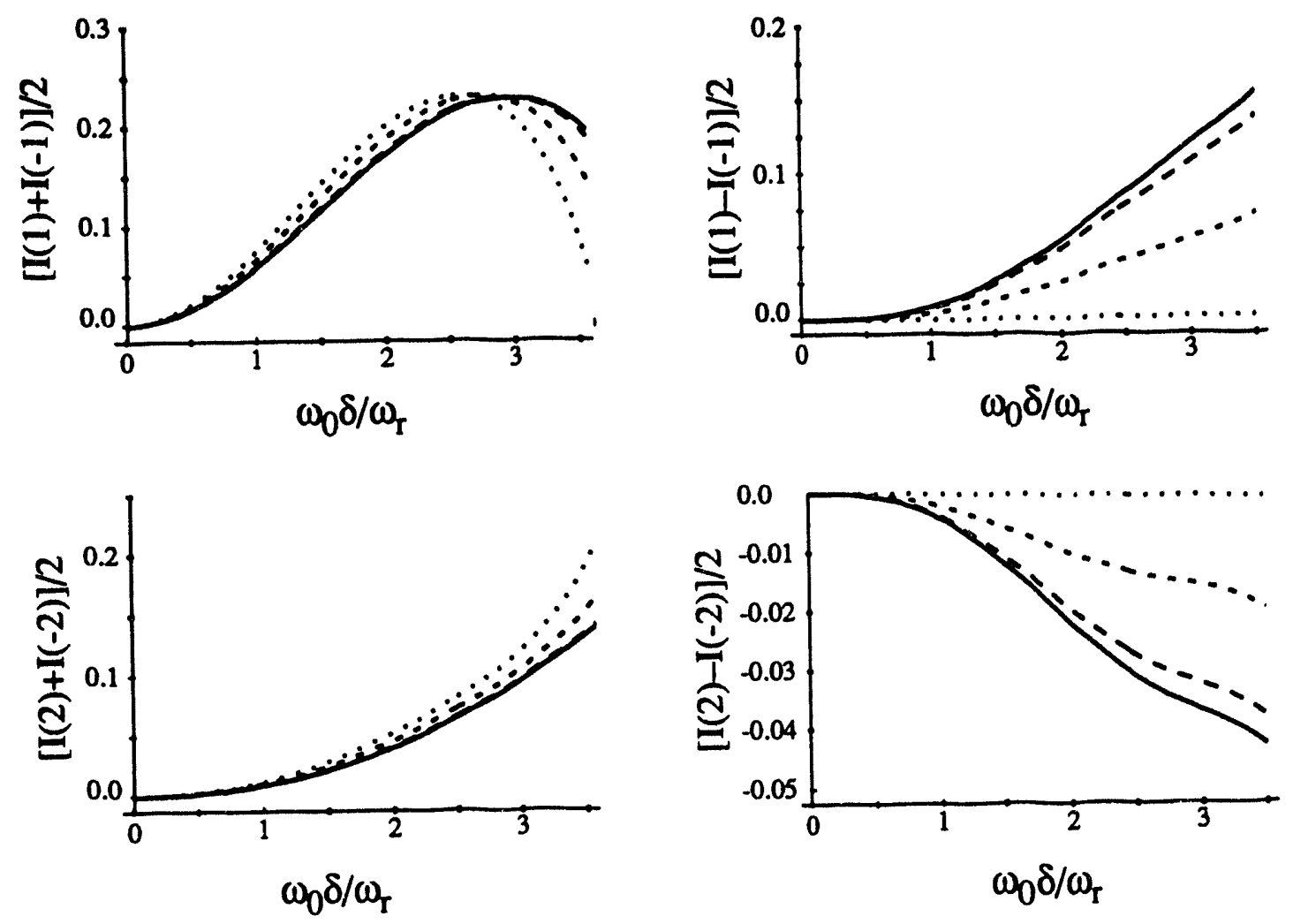

Figure 4.3: Variation of ratios of the differences and averages of the sideband intensities to the centerband intensity with the ratio of the anisotropy $(\delta)$ to spinning speed, $\frac{\omega_{0} \delta}{\omega_{r}}$, and the asymmetry factor $(\eta)$. The four curves correspond to $\eta=0$ (solid line), $\eta=0.3$ (long dashed line), $\eta=0.7$ (short dashed line), and $\eta=1.0$ (dotted line). 
less accurate than that of the anisotropy $\delta$.

The overall quality of the fitting can be seen in Table (4.1). One severe problem appears when the asymmetry factor $\eta$ is very small (that is, in the near-axial regime). Here the differences of the sideband intensities for different $\eta$ are so small (see also

Table 4.1: Results of sideband analysis

\begin{tabular}{lcc}
\hline \hline & $\delta(\mathrm{ppm})$ & $\eta$ \\
\hline Lead Nitrate $\left(\mathrm{PbNO}_{3}\right)\left({ }^{207} \mathrm{~Pb}\right)$ & & \\
$\quad$ Reported & $35.4 \pm 3$ & $0.0 \pm 0.16$ \\
Powder Lineshape & $35.9 \pm 5$ & 0.0 \\
$\quad$ Sideband & $34.6 \pm 5$ & $0.08 \pm 0.1$ \\
Benzoic Acid $\left({ }^{13} \mathrm{C}\right)$ & & \\
Reported & $71.0 \pm 4$ & $0.6 \pm 0.12$ \\
Sideband & $63.4 \pm 5$ & $0.8 \pm 0.1$ \\
Phoshous Pentaoxide $\left({ }^{31} \mathrm{P}\right)$ & & \\
Reported & $218.0 \pm 20$ & $0.0 \pm 0.18$ \\
Sideband & $190.6 \pm 5$ & $0.06 \pm 0.1$ \\
Powder Lineshape & $193.0 \pm 5$ & 0.0 \\
\hline
\end{tabular}

Figure 4.3) that extraction of $\eta$ becomes quite difficult. Such a problem exists both for moment analysis and powder lineshape simulation[97] methods.

This approximate method also can be applied to calculate the centerband intensity after all sidebands are suppressed by a TOSS pulse sequence[99]. The FID signal of a spectrum with sidebands contains a series of rotational echoes. Moreover the rotational echo results from the periodicity of the phase, which runs from 0 to $2 \pi$ over time. After TOSS, however, this period no longer exists[100], and then Eq. (4.8) must be replaced by

$$
\phi_{m}=\exp \left(-i m \omega_{r} t\right)
$$




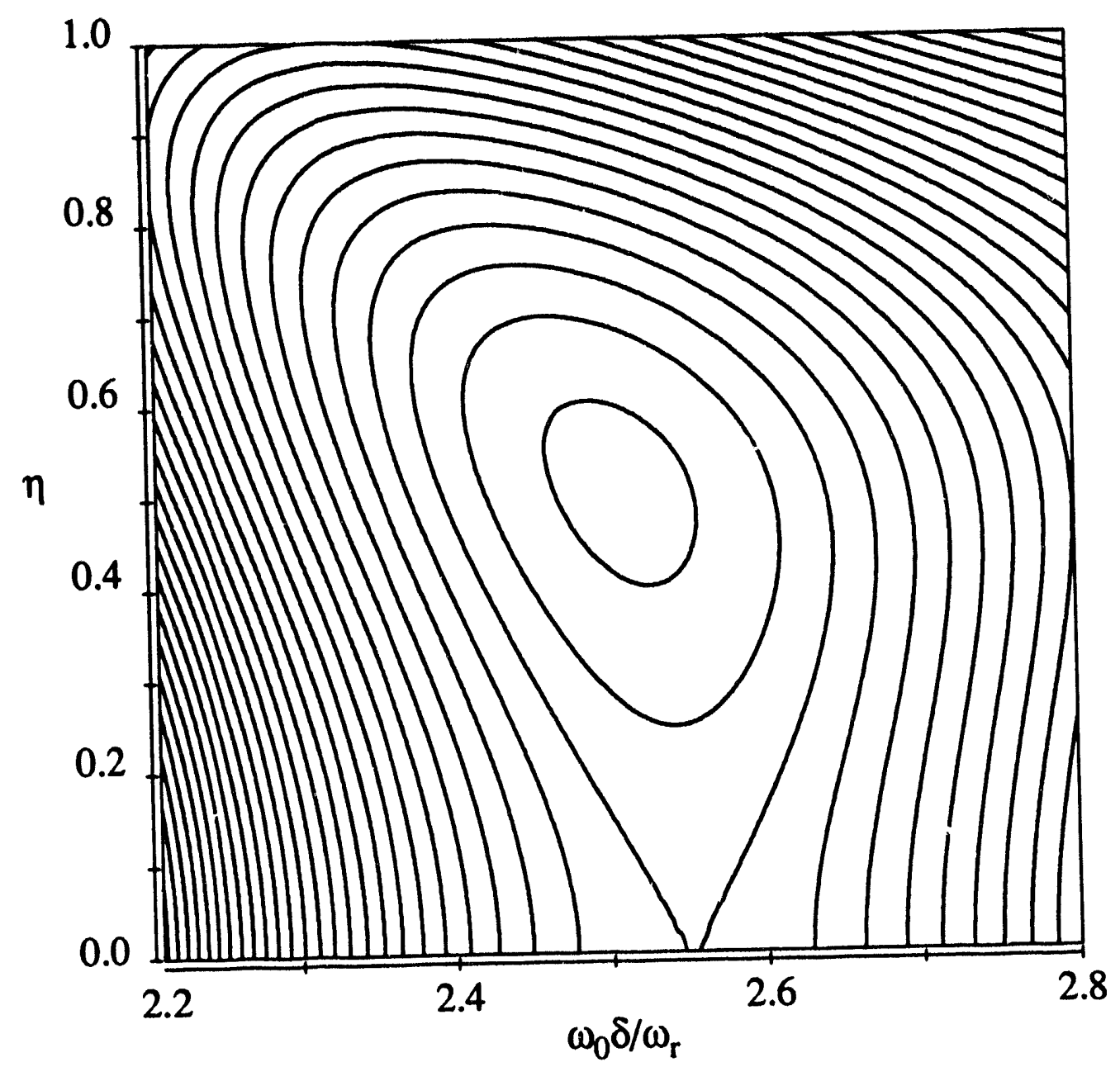

Figure 4.4: Contour of the $\delta-\eta$ surfacs used in the least square fitting program, varying with the ratio of the anisotropy $(\delta)$ to spinning speed, $\frac{\omega_{0} \delta}{\omega_{r}}$, and the asymmetry factor $(\eta)$. The minimum corresponds to $\frac{\omega_{0} \delta}{\omega_{r}}=2.5$, and $\eta=0.5$. 


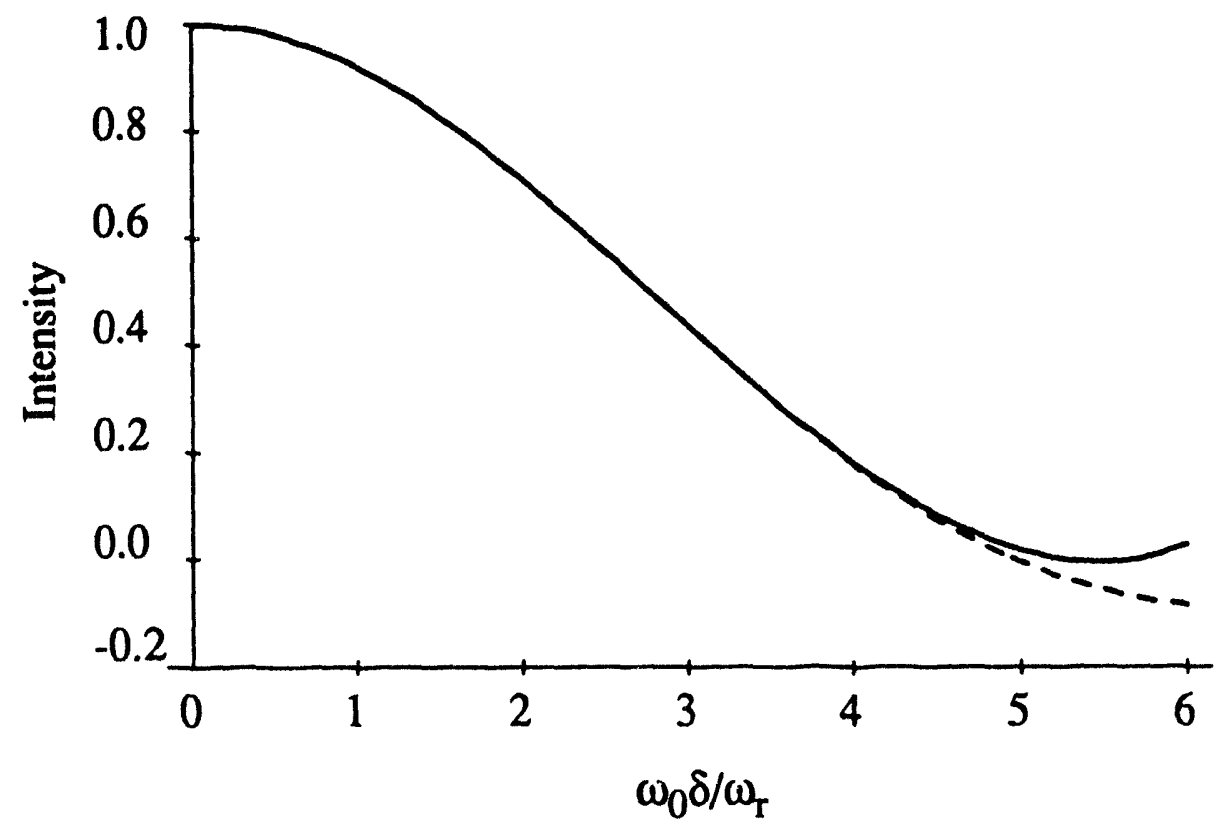

Figure 4.5: Variation of centerband intensity with the ratio of the anisotropy $(\delta)$ to spinning speed, $\frac{\omega_{0} \delta}{\omega_{r}}$, at $\eta=0.5$ after application of a TOSS pulse sequence. 
This expression becomes $\phi_{m_{1}} \phi_{m_{2}} \ldots \phi_{m_{k}}=\exp \left(m_{1}+m_{2}+\ldots+m_{k}\right)=1$ after the powder average. Thus $\overline{f_{k}(t)}$ becomes time independent, and $\overline{g(t)}$ gives the intensity of the centerband. From Eq. (4.18) and the properties of the Clebsch-Gordan coefficients, it can be found that $\overline{f_{k}(t)}$ are zero if $\mathrm{k}$ is odd number. Figure (4.5) shows the variation of the centerband intensity with the ratio of the anisotropy to the spinr

speed, $\frac{\omega_{0} \delta}{\omega_{r}}$, at $\eta=0.5$ in a TOSS experiment. It can be seen that, after TOSS, centerband intensity converges much faster than MAS centerband intensity.

\subsubsection{Conclusions}

We have shown that the FID signal under MAS can be expanded into a Taylor series which contains products of $k$ irreducible spatial tensors $(k=0 \ldots \infty)$. The properties of irreducible tensors permit the integrals over all orientations to be solved analytically. The FID signal then becomes an expansion in a set of basis scalar operators, which are uniquely determined by the tropic parameters of the CSA tensors, and the coefficients in the expansion art $\quad y$ a series of Clebsch-Gordan coefficients. After all the coefficients and the scalar operators are evaluated, the sideband intensities are functions of $\eta^{2}$, and the total pattern of sidebands can be understood as a sum of symmetric and antisymmetric parts about the centerband. The odd terms in the expansion determine the antisymmetric pattern, and make no contribution to the centerband intensity, while the even terms contribuie to the symmetric part. After manipulation of the Clebsch-Gordan coefficients, we obtain an approximate formula up to the ninth order in the Taylor expansion of the FID signal. Sideband intensities can be easily calculated within real time using this formula even for the spectra consisting of many deferent sites whose sidebands overlap with each other. The results are in satisfactory agreement with the exact solution obtained by numerical simulation if the ratio of the anisotropy to the spinning speed, $\frac{\omega_{0} \delta}{\omega_{r}}$, is smaller than three. The anisotropic parameters can be extracted very efficiently using this method combining with least-squares fitting methods. We also apply this 
method to calculate the centerband intensity after eliminating the sidebands using TOSS pulse sequence in MAS. The results show that the centerband intensity after TOSS converges to the exact value much faster than MAS centerband intensity. This approximate method also can be applied to sideband intensity calculation in double rotation (DOR) [72, 104, 105], and dynamical angle spinning (DAS)[76] NMR

\subsection{Sidebands in Double Rotation (DOR) NMR}

\subsubsection{Introduction}

For half integer spin nuclei, the dispersion of the central transitions $\left(\frac{1}{2} \leftrightarrow-\frac{1}{2}\right)$ for a polycrystalline or amorphous sample mainly comes from the second order effects of the quadrupolar interactions[101, 102, 103]. Such a dispersion makes NMR spectra featureless owing to the overlap of lineshapes resulting from different sites in the sample, leading to a major obstacle in the applications of high resolution solid state NMR to a large class of these nuclei. Theoretically the dispersion of a particular nuclear transition is determined by the orientation-dependent resonance frequencies of the magnetization with respect to the external magnetic field. The orientation dependence of the central transition frequencies arising from the second order effects of the quadrupolar interactions cab be described by the linear combination of a second- and a fourth-rank spatial tensor (see Eq. 3.8 or 3.20), and thus cannot be removed completely by the conventional magic angle spinning (MAS) method[56, 58] (see Figure 3.4). Recently, it has been proven experimentally that double rotation (DOR) $[72,78,104]$ as well as dynamical angle spinning (DAS) [76, 73] are the right solutions to suppress the second-order anisotropic broadening and yield high resolution spectra. In DOR, a small inner rotor is embedded in a large outer rotor (see Figure 3.23). The outer rotor spin around an axis inclined at $\theta^{(2)}=54.74^{\circ}$ (the magic angle of the second-order Legendre polynomial) with respect to the external magnetic field while the inner rotor rotates around another axis tilted at $\theta^{(4)}=30.56^{\circ}$ 
(one of the magic angles of the fourth-order Legendre polynomial) relative to the rotation axis of the outer-rotor.

From section 3.8, all the anisotropies of the interactions can be effectively averaged to zero by DOR if the ratio of the inner rotor spinning speed to outer rotor spinning speed is larger than four, and if the spinning speeds of both rotations are much larger than the amplitude of the internal anisotropic interactions (chemical shift anisotropy, quadrupole, and dipole). However, owing to the mechanical limitation of sample spinning system, the typical spinning speed of the outer rotor is about one kilo-Herz or less while the spinning speed of the inner rotor reaches five kilo-Herz. It is obvious that the spinning speeds of both the inner and outer rotors are not in the fast spinning region. Since the second order quadrupolar broadening is still inhomogeneous, using DOR will, therefore, yield high resolution spectra accompanied by a train of sidebands. Although the appearance of the DOR sidebands makes the assignment of the spectra difficult, a cheap way to extract anisotropic information from the DOR sidebands is provided through the analysis of the DOR sideband intensities $[96,106]$.

In this section, the sidebands in the DOR spectra are analyzed by the moment method proposed by Maricq and Waugh and also the Bessel function method used by Herzfeld and Berger in MAS sideband analysis. General formulae for the calculations of the moments and sideband intensities are derived. Unlike in MAS, the sideband intensities depend on not only the anisotropic parameters $(\delta$ and $\eta)$ and the spinning speed of the outer rotor, but also the ratio of spinning speeds of the inner rotor to the outer rotor as well as the relative rotor phase between the inner and outer rotors. The sideband patterns with a particular rotor phase is more sensitive than the average over the rotor phases, similar to the difference between the sidebands arising from a single crystal and those from a powder sample. Finally, numerical simulations are implemented and shown to agree with experimental results. Anisotropy information 
of the quadrupolar interaction therefore can be recovered by simulating the sideband intensities or the rotor phase dependence of the sideband intensities.

\subsubsection{Experimental Results}

Figure (4.6) shows the experimental spectra of sodium-23 nuclei in sodium oxalate with quadrupolar coupling constant $2.43 \mathrm{MHz}$ and asymmetry parameter 0.72 under DOR. It can be clearly seen that the spectra consist of high-resolution isotropic peaks accompanied with a train of sidebands like those under MAS. However the envelop of sidebands does not mimic the static powder pattern (a feature observed in a MAS spectra under slow spinning condition). This can be explained as follows. The envelop of sidebands of the first rotation spinning at the magic angle $\theta^{(2)}$, forms a $P_{4}$ lineshape meanwhile the envelop of sidebands of the second rotation spinning at $\theta^{(4)}$ forms a $P_{2}$ lineshape. The total envelop of all sidebands is the convolution of these two lineshapes. When the inner rotor does not spin fast enough, the total envelop will be severely distorted.

In addition, the frequency difference between two nearest sidebands in a MAS spectrum is uniquely determined by the spinning speed $\omega_{r}$ of the sample. However in DOR spectra there are total eighty-one different sets of sidebands in principle. Each of them has the frequency difference of $m_{1} \omega_{r 1}+m_{2} \omega_{r 2}$ corresponding to a particular pair of $m_{1}, m_{2}$ values from -4 to 4 . All sets of sidebands overlap each other and make the pattern of sidebands complicated. Experimentally, since the inner-rotor spins much faster than the outer rotor, the intensity of sidebands are mainly determined by the outer rotor spinning speed.

\subsubsection{FID Signal under DOR}

After applying two simultaneous rotations to the sample with half integer spin nuclei, the time dependence of the central transition frequencies arising from the second 

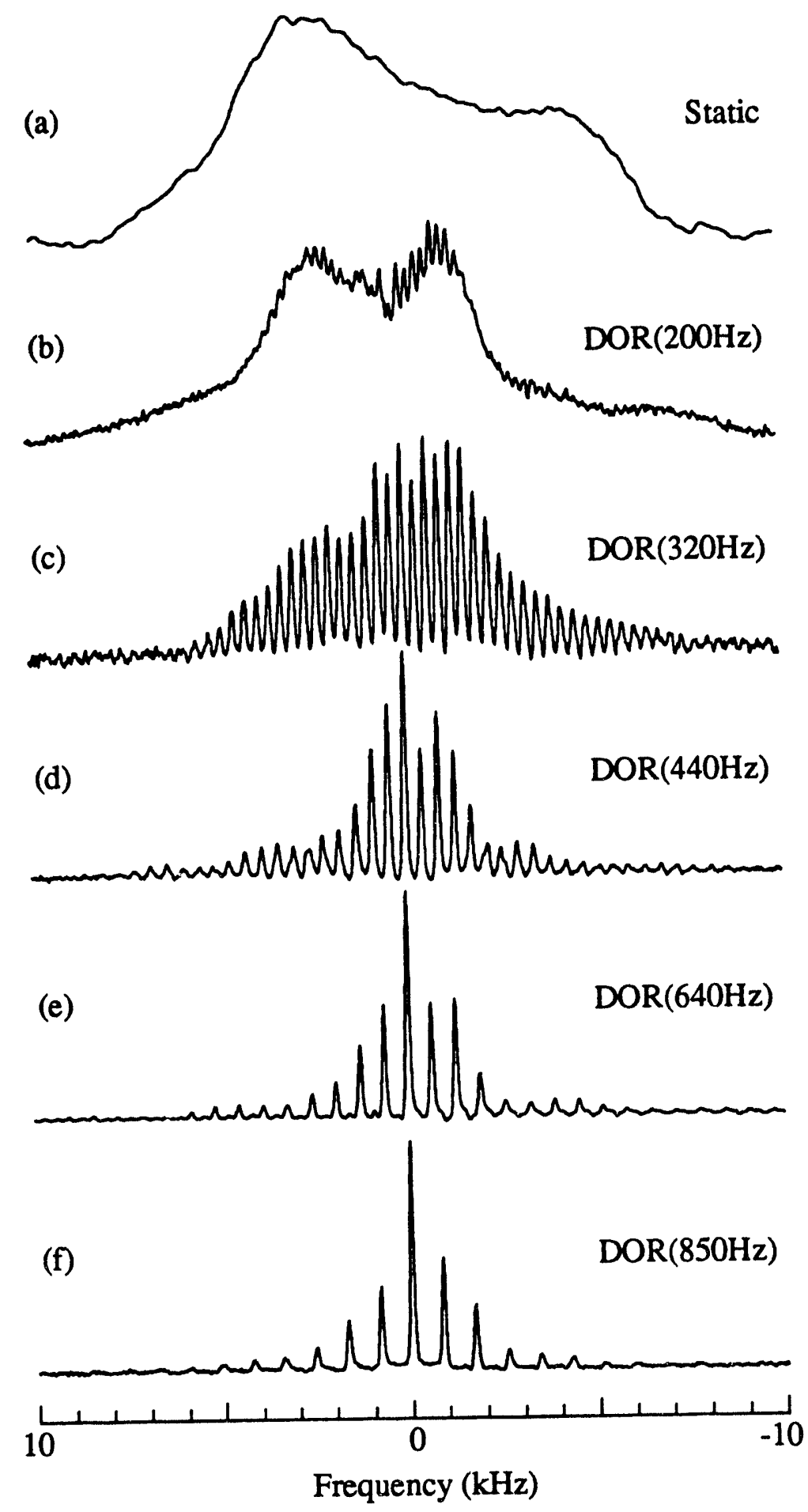

Figure 4.6: Variation of sidebands measured experimentally in sodium-23 spectra of sodium oxalate with different spinning speed under double rotation (DOR). The quadrupolar coupling constant is $\omega_{Q}=405 \mathrm{kHz}$, the asymmetry parameter, $\eta$, is 0.72 , and the Larmor frequency of the sodium nuclei is $105.8 \mathrm{M} \mathrm{Hz}$. 
order effects of the quadrupolar interactions is given by Eq.(3.45) or

$$
\omega_{\frac{1}{2} \leftrightarrow-\frac{1}{2}}^{(2)}(t)=\sum_{l=0,2,4} C_{l} \sum_{m_{1}, m_{2}=-l}^{l} \mathcal{D}_{m_{1}, 0}^{(l)}\left(\Omega_{r_{1}}(t)\right) \mathcal{D}_{m_{2}, m_{1}}^{(l)}\left(\Omega_{r_{2}}(t)\right) A_{l m_{2}},
$$

where $C_{l}$ is given by Eq.(3.26) and $\Omega_{r_{1}}(t)$ and $\Omega_{r_{2}}(t)$ are two sets of Euler angles defined by Eq.(3.46). If we assume an on-resonance condition, the total phase of the magnetization at time $t$ after applying a $90^{\circ}$ pulse is

$$
\varphi(t)=\sum_{m_{1}, m_{2}=-4}^{4} B_{m_{1}, m_{2}} e^{-\imath\left(m_{1} \gamma_{r}+m_{2} \alpha\right)}\left(e^{-\imath\left(m_{1} \omega_{r 1}+m_{2} \omega_{r 2}\right) t}-1\right)
$$

where

$$
B_{m_{1}, m_{2}}=\sum_{l=2,4} \imath C_{l} \frac{d_{m_{1}, 0}^{(l)}\left(\beta_{r 1}\right) d_{m_{2}, m_{1}}^{(l)}\left(\beta_{r 2}\right)}{m_{1} \omega_{r 1}+m_{2} \omega_{r 2}} \sum_{m^{\prime}} d_{m^{\prime}, m_{2}}^{(l)}(\beta) \rho_{l m^{\prime}} e^{-\imath m^{\prime} \gamma}
$$

and the Euler angles, $(\alpha, \beta, \gamma)$, are used to describe the orientation dependence of a single spin nucleus in the inner-rotor frame. The FID signal therefore can be simply written as

$$
g(t)=e^{-\imath \varphi(t)}
$$

For a powder sample, the average over all orientations may be implemented by performing a three-dimensional integral over Euler angles, that is

$$
G(t)=\frac{1}{8 \pi^{2}} \int_{0}^{2 \pi} d \alpha \int_{0}^{2 \pi} d \gamma \int_{0}^{\pi} \sin \beta d \beta g(t)
$$

It may be worthwhile to point out that the $\pi$ symmetry for $\alpha$ integral and half $\pi$ symmetry for $\beta$ integral are totally destroyed here due to incomplete truncation of the sample rotation.

\subsubsection{Moment Analysis of DOR Sidebands}

Moment analysis method was first proposed by van Vleck[107] in order to characterize powder lineshapes in NMR spectra arising from homogeneous spin interactions, for example, the dipolar couplings between like-spin nuclei. Later Maricq and Waugh[95] had applied this method to analyze the sidebands in MAS spectra. The moments 
calculated from the sideband intensities and the spinning speeds in MAS spectra can then be used to extract the chemical shift anisotropy parameters $\delta$ and $\eta$. Here we can also adapt this moment analysis method to analyze the DOR sidebands, and to extract, if possible, the anisotropic parameters of the quadrupolar interactions. We first introduce the definition of the $n$-th moment given by

$$
M_{n}=\int_{-\infty}^{\infty} d \omega \omega^{n} G(\omega)=\sum_{N=-\infty}^{\infty} \omega_{N}^{n} I_{N}
$$

where $\omega_{N}$ is the resonance frequency of the $N$-th sidebands, $I_{N}$ is the intensity, and $G(\omega)$ is the spectrum given by $G(\omega)=\sum_{N} I_{N} \delta\left(\omega-\omega_{N}\right)$, resulting from Fourier transformation of the FID signal $G(t)$ without relaxation broadenings. Once the spectrum is measured experimentally, the moments can be calculated by use of Eq.(4.31).

In order to relate the moments with the anisotropic parameters of the quadrupolar interaction, it is possible to derive a relationship between the FID signal and the moments given by [5]

$$
G(t)=\sum_{n=0}^{\infty} \frac{(-\imath t)^{n}}{n !} M_{n} .
$$

The inverse expression of Eq.(4.32) gives the $n$-th moment

$$
M_{n}=\left.\imath^{n} \frac{d^{n}}{d t^{n}} G(t)\right|_{t=0}
$$

Inserting Eq.(4.30) into Eq.(4.33) and then taking an average over all Euler angles yield the first few moments. In order to represent the complicated equations, we first introduce following definition:

$$
\begin{aligned}
& P_{C G}\left[p_{k-3}(L) p_{k}(l) p_{k-1}(m)\right] \equiv C\left(l_{1} l_{2} L_{1}, m_{1}, m_{2}\right) C\left(L_{1} l_{3} L_{2}, m_{1}+m_{2}, m_{3}\right) \cdots \\
& \times C\left(L_{k-3}, l_{k-1}, l_{k}, \sum_{i=1}^{k-2} m_{i}, m_{k-1}\right) C\left(l_{k} l_{k} 0, \sum_{i=1}^{k-1} m_{i},-\sum_{i=1}^{k-1} m_{i}\right) \\
& p_{k}(l)=l_{1} \cdots l_{k}
\end{aligned}
$$

where $C\left(l_{1} l_{2} l_{3}, m_{1} m_{2}\right)$ are the Clebsch-Gordan coefficients. The first four moment can now be written as

$$
M_{0}=1
$$




$$
\begin{aligned}
M_{1} & =0 \\
M_{2} & =\sum_{p_{2}(l)} a_{2}\left[p_{2}(l)\right] \rho_{00}\left[p_{2}(l)\right] \\
M_{3} & =\sum_{p_{2}(l)} a_{3}\left[p_{2}(l)\right] \rho_{00}\left[p_{2}(l)\right]+\sum_{p_{3}(l)} a_{3}\left[p_{3}(l)\right] \rho_{00}\left[p_{3}(l)\right] \\
M_{4} & =\sum_{p_{2}(l)} a_{4}\left[p_{2}(l)\right] \rho_{00}\left[p_{2}(l)\right]+\sum_{p_{3}(l)} a_{4}\left[p_{3}(l)\right] \rho_{00}\left[p_{3}(l)\right] \\
& +\sum_{p_{1}(L) p_{4}(l)} a_{4}\left[p_{1}(L) p_{4}(l)\right] \rho_{00}\left[p_{1}(L) p_{4}(l)\right]
\end{aligned}
$$

there $p_{k}(l)=l_{1} l_{2} \cdots l_{k}$. The scalar operators (or zero rank irreducible tensors), $\rho_{00}\left[p_{k}(l)\right]$, arising from the product of $k$ spatial irreducible tensors, $A_{l_{i}, m_{i}}$ for $i=$ $1 \cdots k$, are given by

$$
\rho_{00}\left[p_{k-3}(L), p_{k}(l)\right]=\sum_{p_{k-1}(m)} P_{C G}\left[p_{k-3}(L) p_{k}(l) p_{k-1}(m)\right] \rho_{l_{1} m_{1}} \cdots \rho_{l_{k-1} m_{k-1}} \rho_{l_{k},-\sum_{i=1}^{k-1} m_{i}},
$$

and $\rho_{l, m}$ are the principal values of the second order quadrupolar coupling tensors shown in Eq.(3.12). The coefficients of the scalar operators in the moment expansion can be expressed as

$$
\begin{aligned}
& a_{k}\left[p_{k-3}(L) p_{k}(l)\right]=\sum_{p_{k}(m) p_{k-1}(n)} d_{k}\left[p_{k-3}(L) p_{k}(l) p_{k}(m) p_{k-1}(n)\right] \\
& a_{3}\left[p_{2}(l)\right]=\sum_{p_{2}(m) p_{1}(n)} 3\left(m_{2} \omega_{r_{1}}-n_{1} \omega_{r_{2}}\right) d_{2}\left[p_{2}(l) p_{2}(m) p_{1}(n)\right] \\
& a_{4}\left[p_{2}(l)\right]=\sum_{p_{2}(m) p_{1}(n)}\left[4\left(m_{1} \omega_{r_{1}}+n_{1} \omega_{r_{2}}\right)+3\left(m_{2} \omega_{r_{3}}-n_{1} \omega_{r_{2}}\right)\right] d_{2}\left[p_{2}(l) p_{2}(m) p_{1}(n)\right] \\
& a_{4}\left[p_{3}(l)\right]=\sum_{p_{3}(m) p_{2}(n)} 6\left[m_{3} \omega_{r_{1}}-\left(n_{1}+n_{2}\right) \omega_{r_{2}}\right] d_{3}\left[p_{3}(l) p_{3}(m) p_{2}(n)\right] \\
& d_{k}\left[p_{k-3}(L) p_{k}(l) p_{k}(m) p_{k-1}(n)\right]=C_{l_{1}} \cdots C_{l_{k}} d_{m_{1}, 0}^{\left(l_{1}\right)}\left(\theta_{1}\right) \cdots d_{m_{k}, 0}^{\left(l_{k}\right)}\left(\theta_{1}\right) \\
& \times d_{n_{1}, m_{1}}^{\left(l_{1}\right)}\left(\theta_{2}\right) \cdots d_{n_{k}, m_{k}}^{\left(l_{k}\right)}\left(\theta_{2}\right) P_{C G}\left[p_{k-3}(L) p_{k}(l) p_{k-1}(n)\right] \\
& n_{k}=\sum_{i=1}^{k} n_{k} .
\end{aligned}
$$

As can be seen from Eqs.(4.35), the $n$-th moment is represented in a set of zero rank irreducible tensors (or scalar operators), $\rho_{00}\left[p_{k-3}(L) p_{k}(l)\right]$, for $k=2 \cdots n$. Each scalar operator arises from the product of $k$ second order quadrupolar coupling 
tensors, $\rho_{l_{i}, m_{i}}$, for $i=2 \cdots k$. Actually, the representation of the $n$-th moment in the set of scalar operator given in Eqs.(4.35) is also true for the static situation, but the $n$-th moment is only related to a single scalar operator which is the product of $n$ second order quadrupolar coupling tensors. The sample spinning therefore results in the mixing between the static moments with the sample spinning speeds.

The anisotropic parameters $\delta$ and $\eta$ of the quadrupolar interaction are encoded in the zero rank tensors. From the definition of the principal values of the second order quadrupolar coupling tensors given by Eq.(3.12), the zero rank tensor can be expanded into

$$
\rho_{00}\left[p_{3-k}(L) p_{k}(l)\right]=\delta^{2 k} \sum_{i=0}^{k} b_{i}\left[p_{3-k}(L) p_{k}(l)\right] \eta^{2 i}
$$

where

$$
\begin{aligned}
b_{i}\left[p_{k-3}(L) p_{k}(l)\right] & =\sum_{p_{k-1}(n), p_{k}(m)} P_{C G}\left[p_{k-3}(L) p_{k}(l) p_{k-1}(n)\right] C\left(22 l_{1}, m_{1}, n_{1}-m_{1}\right) \cdots \\
& \times C\left(22 l_{k}, m_{k}, n_{k}-m_{k}\right) \rho_{2, m_{1}} \rho_{2, n_{1}-m_{1}} \cdots \rho_{2, m_{k}} \rho_{2, n_{k}-m_{k}},
\end{aligned}
$$

with the conditions of

$$
\begin{aligned}
n_{k} & =-\sum_{j=1}^{k-1} n_{j} \\
2 i & =\sum_{j=1}^{k}\left(\left|m_{j}\right|+\left|n_{j}-m_{j}\right|\right),
\end{aligned}
$$

and $\rho_{2, m}$ are principal values of the quadrupolar coupling tensor with $\delta=1$ and $\eta=1$.

The fourth and higher moments do not contain any new information which is not already available from the second and third moments. The anisotropic parameters therefore may be calculated from Eqs.(4.38) after the second and the third moments are obtained from the experimental DOR spectra. It has been shown by Maricq and Waugh[95] that the second and the third moments obtained from the MAS spectra are independent of the spinning speed and the fourth and higher moments increases with $\omega_{r}$. However in the DOR case, only the second or lower moments 
are independent of the spinning speeds and the third or higher moments increase with $\omega_{r_{1}}$ and $\omega_{r_{2}}$. This explains why the envelop of the sidebands does not mimic the static powder lineshape and changes with the spinning speeds because the third moment determines the asymmetry properties around the isotropic frequencies of the lineshapes. In the MAS case, the parameter $\mu=M_{4} / M_{2}^{2}$, which characterizes the lineshapes, increases with $\omega_{r}^{2}$, leading to quasi-Lorentzian lines of decreasing halfwidth of the envelop. In the DOR case, the parameter $\mu$ increases with the spinning speeds $\omega_{r_{1}}$ and $\omega_{r_{2}}$ linearly and bilinearly. This means that the halfwidth of the envelop of the DOR sidebands decreases according to quasi-super-Lorenrzian lines. This feature can be seen from Figure (4.6).

It is general that all moments except the zero and first moments are dependent on the relative rotor phase $\gamma_{r}$. Introduction of this phase dependence in the moment calculation does not bring any new information and makes the whole expression more complicated. Experimentally by randomly taking the relative phase at different times, the relative phase effect may be averaged out. This ?veraging process is equivalent to taking an integral over the relative phase in Eqs.(4.38) to yield $\sum_{i=1}^{k} m_{i}=0$.

\subsubsection{Bessel analysis of DOR sidebands}

The moment analysis may have severe problem in practical cases since in principle an infinite number of sidebands has to be taken into account or else the short time behavior of the rotational spin echoes has to be analyzed very accurately. The solution to overcome this problem is to use Bessel function analysis of the sideband intensities developed first by Herzfield and Berger.

From the properties of Bessel functions $\left\{J_{k}(z)\right\}$, we can derive

$$
e^{-\imath z e^{-\imath \theta}}=\sum_{k} \mathcal{J}(z) e^{-\imath k \theta}
$$


where

$$
\mathcal{J}_{k}(z)=\sum_{k^{\prime}}(-\imath)^{k^{\prime}} J_{k^{\prime}}(z) J_{k-k^{\prime}}(-\imath z)
$$

and $z$ is a complex number. The inverse transformation of Eq. (4.41) is

$$
\mathcal{J}_{k}(z)=\frac{1}{2 \pi} \int_{0}^{2 \pi} e^{z\left(k \theta-z e^{-s \theta}\right)} d \theta .
$$

Now, by use of Eq. (4.41) FID signal arisirg from the central transitions can be expressed

$$
\begin{aligned}
g(t) & =\left\{\prod_{m_{1}, m_{2}=-4}^{4} \sum_{K_{m_{1}, m_{2}}^{1}} \sum_{K_{m_{1}, m_{2}}^{2}} \mathcal{J}_{K_{m_{1}, m_{2}}^{1}}\left(B_{m_{1}, m_{2}}\right) \mathcal{J}_{K_{m_{1}, m_{2}}^{2}}\left(-B_{m_{1}, m_{2}}\right)\right\} \\
& \times e^{\left.-\mathfrak{t}\left(N_{r_{1}} \omega_{r_{1}}+N_{r_{2}} \omega_{r_{2}}\right) t+\gamma_{r}\left(N_{r_{1}}^{1}+N_{r_{1}}^{2}\right)+\alpha\left(N_{r_{2}}^{1}+N_{r_{2}}^{2}\right)\right\}}
\end{aligned}
$$

where

$$
\left\{\begin{array}{l}
N_{r 1}^{i}=\sum_{m_{1}, m_{2}} m_{1} K_{m_{1}, m_{2}}^{i} \\
N_{r 2}^{i}=\sum_{m_{1}, m_{2}} m_{2} K_{m_{1}, m_{2}}^{i},
\end{array}\right.
$$

for $i=1,2$.

Averaging of Eq. (4.44) over $\alpha$ and then using of the inverse transformation in Eq.(4.43) yields

$$
G(t)=\frac{1}{2 \pi^{2}} \sum_{N_{r 1}, N_{r 2}} \int_{0}^{\pi} \sin \beta d \beta \int_{0}^{2 \pi} d \gamma I_{N_{r 1}, N_{r 2}} e^{-\imath\left(N_{r 1} \omega_{r 1}+N_{r 2} \omega_{r 2}\right) t}
$$

where

$$
I_{N_{r 1}, N_{r 2}}=F_{N_{r 1}, N_{r 2}} \sum_{N_{r}} F_{N_{r}, N_{r 2}}^{*} e^{-\imath\left(N_{r 1}-N_{r}\right) \gamma_{r}}
$$

and

$$
F_{N_{r 1}, N_{r 2}}=\frac{1}{(2 \pi)^{2}} \int_{0}^{2 \pi} d \theta_{1} \int_{0}^{2 \pi} d \theta_{2} \exp \left\{-\imath\left[N_{r 1} \theta_{1}+N_{r 2} \theta_{2}+\sum_{m_{1}, m_{2}=-4}^{4} B_{m_{1}, m_{2}} e^{-\imath\left(m_{1} \theta_{1}+m_{2} \theta_{2}\right)}\right]\right\}
$$

From Eq. (4.47) and (4.48), it can be seen that the phase of sidebands is determined by the relative rotor-phase between two applied rotations. Such a property is different from the MAS case where all sidebands are in phase after averaged over the Euler angle $\alpha$. However the sidebands in DOR spectra will be in phase only after 
averaging Eq.(4.44) over the relative rotor-phase. Experimentally this can be done by accumulating FID signal without synchronizing the outer-rotor. The FID signal under DOR then becomes

$$
\overline{G(t)}=\frac{1}{2 \pi^{2}} \sum_{N_{r 1}, N_{r 2}} \int_{0}^{\pi} \sin \beta d \beta \int_{0}^{2 \pi} d \gamma\left|F_{N_{r 1}, N_{r 2}}\right|^{2} \exp ^{-\imath\left(N_{r 1} \omega_{r 1}+N_{r 2} \omega_{r 2}\right) t},
$$

where the overline of the $G(t)$ indicates the average over the relative rotor-phase.

In the limit that the inner rotor spins infinity fast, the coefficients $B_{m_{1}, m_{2}}$ given in Eq.(4.28) are not equal zero only if $m_{2}=0$. This feature leads to the fact that the integration of Eq.(4.48) over $\theta_{2}$ is equal zero except for $N_{r_{2}}=0$, and that the number of integrations of $F$ reduces to one.

Sideband intensities have been evaluated by numerical integrations of Eqs.(4.46), (4.49), for various cases. Composite ten-point Gaussian (Gauss-Legendre) quadruture has to been used to approximate all integrals over $\alpha, \beta, \gamma$. Owing to complicated orientation dependence (compared with the MAS case), the simulations of sideband intensities are much more time consuming than ones in the MAS case. To overcome such a problem, linear interpolation technique may be adapted [111]. Figure (4.7) shows how the sideband intensities varies with the spinning speed of the outer rotor with different ratio of two spinning speeds after averaging over the relative rotor phase.

\subsubsection{Symmetry Properties of DOR Sidebands}

Both the intensities and the phases of the sidebands in DOR spectra depend on the relative rotor phase $\gamma_{r}$ between the outer rotor and the inner rotor. From the experimental results and simulations, even when the relative rotor phase is zero, there are still phase differences among each individual sidebands. Figure (4.8) shows the variation of the sideband intensities, $I_{N_{1}, N_{2}}$ for $N_{2}=0$, and $N_{1}=0, \pm 1, \pm 2$ with the relative phase $\gamma_{r}$. As can be seen from the figure, both the intensities and the phases of all sidebands vary with $\gamma_{r}$. The intensities of real components of the even 


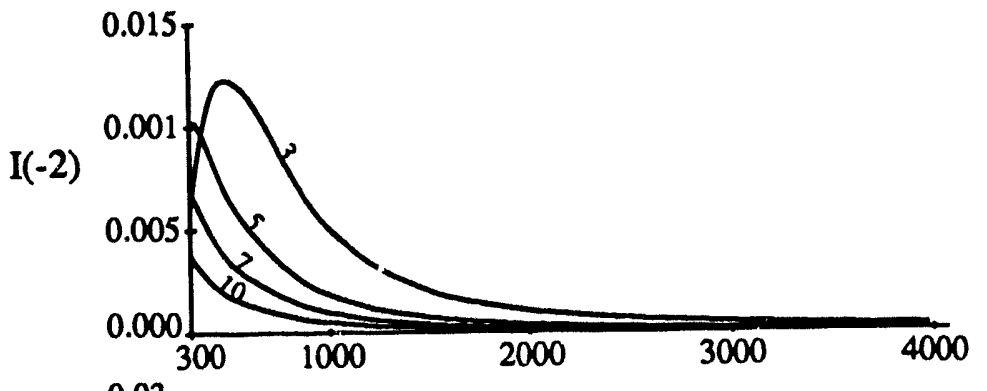

I(-1)
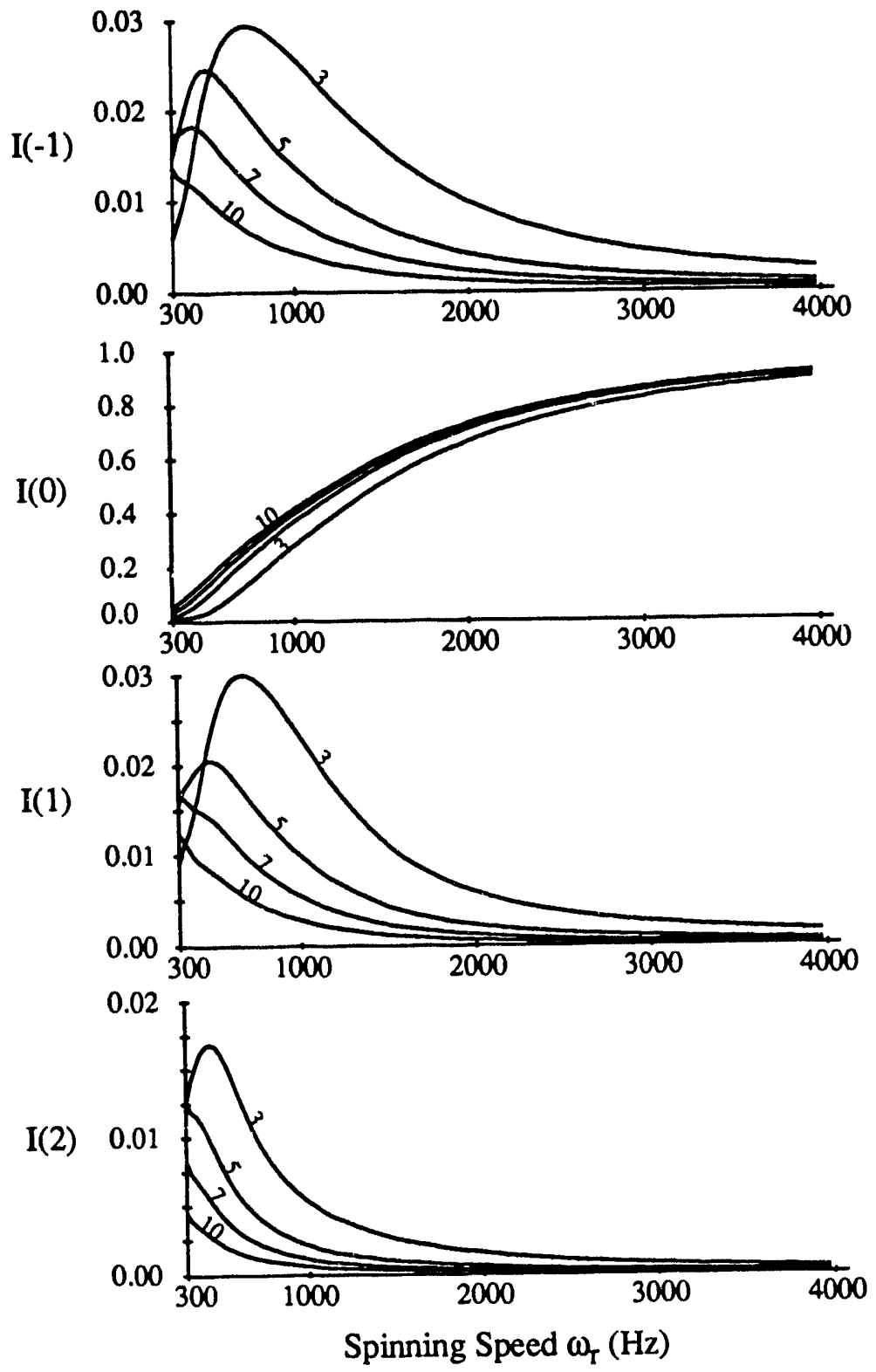

Figure 4.7: Schematic variation of simulated sideband intensities as the spinning speed of the ourter rotor, $\omega_{r_{1}}$, changes with different ratio between the two spinning speeds of the inner and the outer rotors, $\frac{\omega_{r_{2}}}{\omega_{r_{1}}}$, after average over the relative phase $\gamma_{r_{r}}$, where we use the quadrupolar coupling constant and the asymmetry parameter of the sodium-23 nucleus in the sodium oxalate sample, $\omega_{Q}=405 \mathrm{kHz}, \eta=0.72$, and $\omega_{0}=105.8 \mathrm{M} \mathrm{Hz}$. 


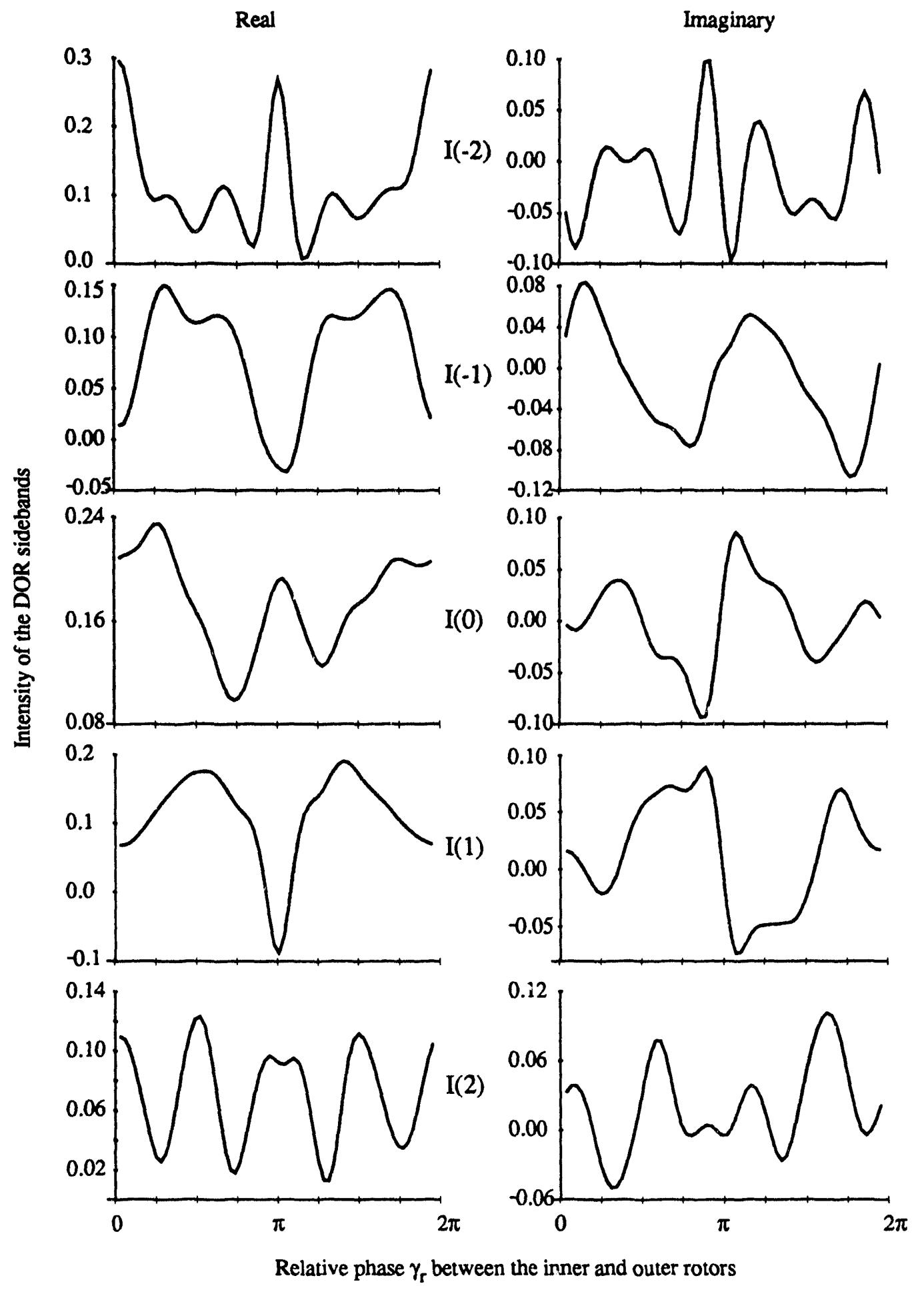

Figure 4.8: Variations of the simulated DOR sideband intensities with different relative rotor phases $\gamma_{r}$ between the inner and outer rotors, where the quadrupolar coupling constant and asymmetry parameter are $\omega_{Q}=405 \mathrm{kHz}$ and $\eta=0.72$, from the sodium oxalate. Larmor frequency is $\omega_{0}=105.8 \mathrm{M} \mathrm{Hz}$, and the outer rotor spinning speed is $700 \mathrm{~Hz}$ while the inner rotor sinning speed is $3.3 \mathrm{kHz}$. 


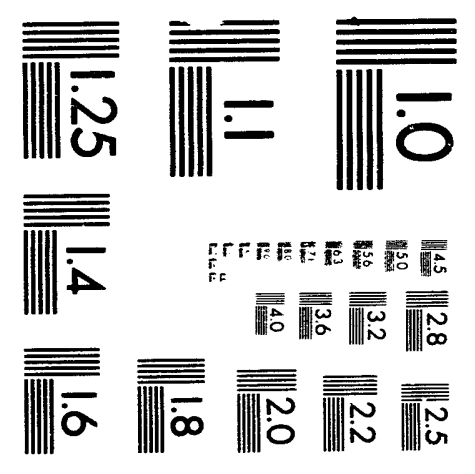



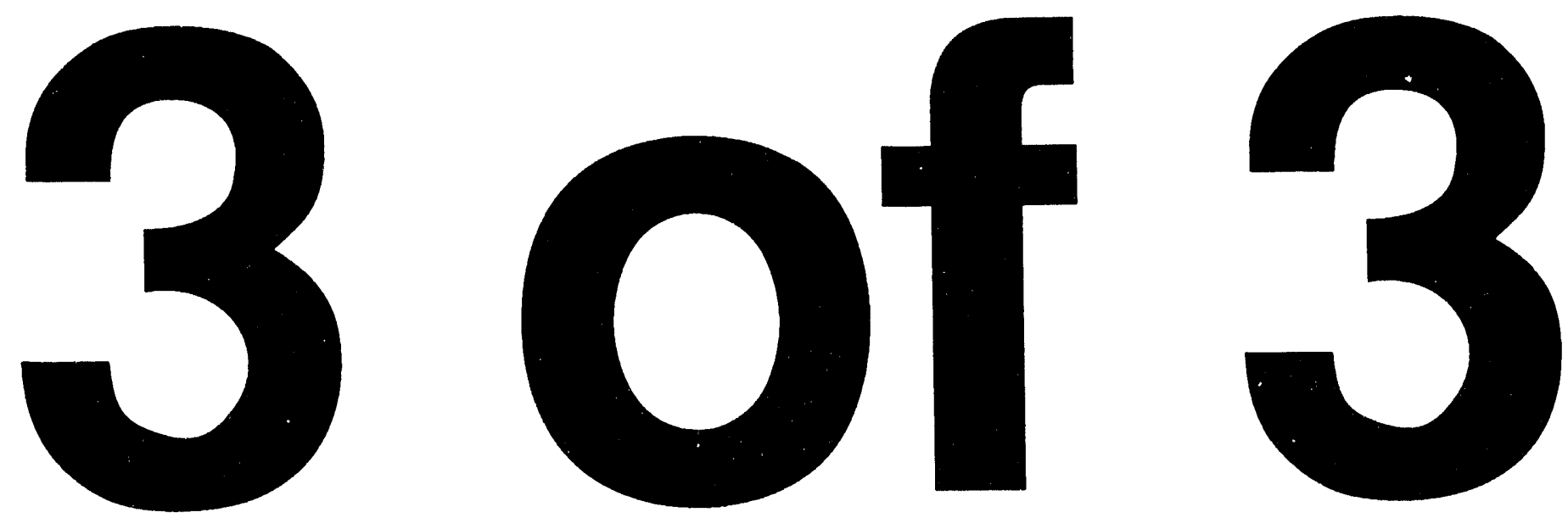


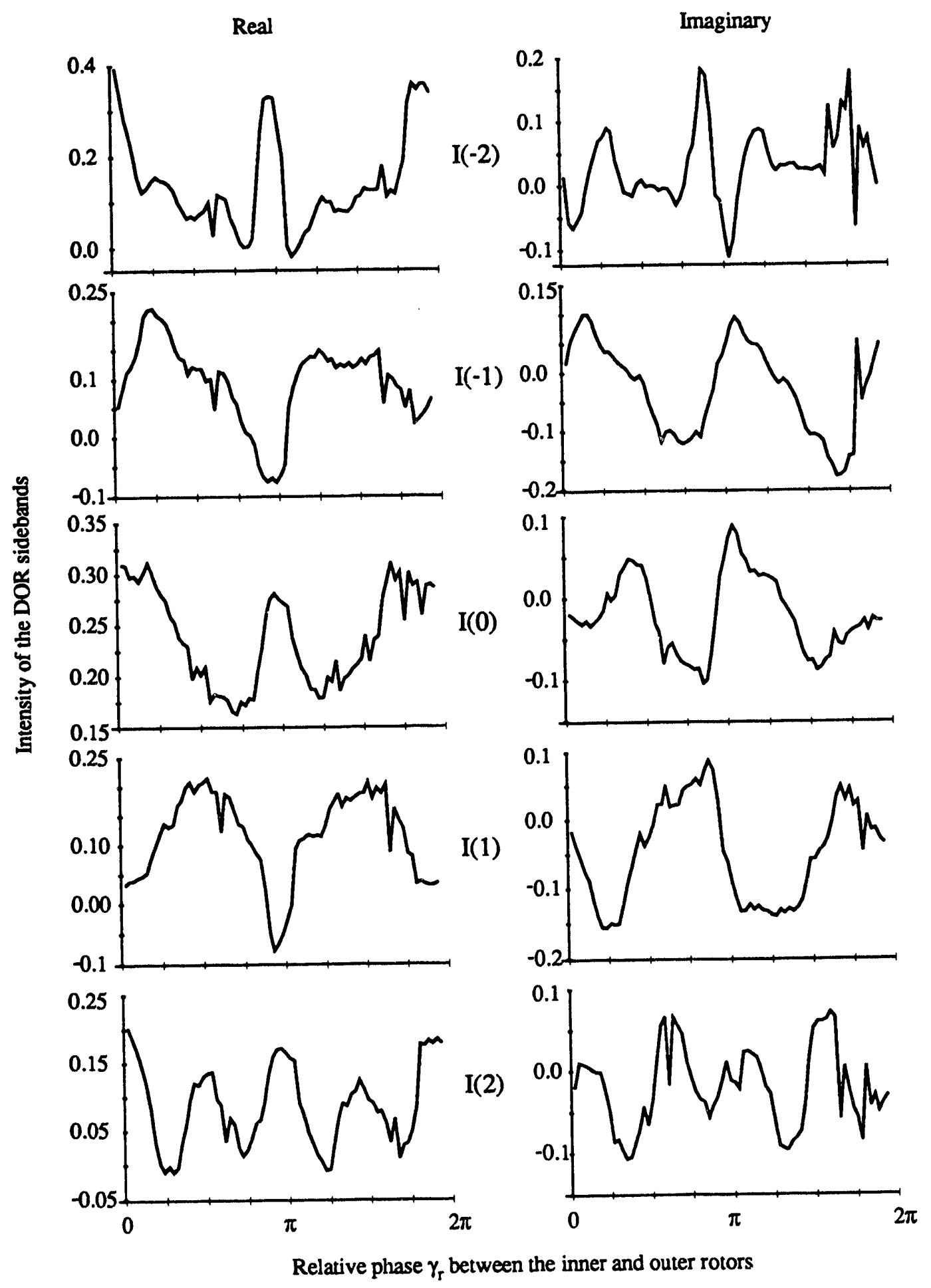

Figure 4.9: Experimental results of the DOR sideband intensities in the sample of sodium oxalate as the relative rotor phase $\gamma_{r}$ between the inner and outer rotors changes. The quadrupolar coupling constant and asymmetry parameter are $\omega_{Q}=405 \mathrm{kHz}$ and $\eta=0.72$; the Larmor frequency is $\omega_{0}=105.8 \mathrm{M} \mathrm{Hz}$; the outer rotor spinning speed is $700 \mathrm{~Hz}$ while the inner rotor rotates at the rate of $3.3 \mathrm{kHz}$. 
order sidebands $\left(N_{1}=0, \pm 2\right.$,etc.) are always positive while the odd order sidebands are oscillating around zero. Especially interesting are the intensities of the odd order sidebands which at $\gamma_{r}=0^{\circ}$ is just opposite to those at $\gamma_{r}=180^{\circ}$. The exact variations of the sideband intensities with the relative rotor phase are determined the anisotropic parameters, $\delta$ and $\eta$, of the quadrupolar interaction as well as the two spinning speeds of the inner and outer rotor. In practice, these variations can be used to extract the anisotropic information by comparing the simulated and experimental results. As an example, Figure (4.9) shows the experimental results of the sideband intensities of the sodium oxalate varying with the relative phase under DOR, in agreement with the simulated results shown in Figure (4.8). The experiment was implemented by synchronizing the outer rotor using a laser sensor. The optical signal detected from the sensor is transferred to TTL pulses using a logical circuit, and then the TTL pulses are used to trigger the pulse program of the spectrometer. By changing the triggering times, we are able to vary the rotor phase from $0^{\circ}$ to $360^{\circ}$. Actually the extraction of the principal values of the quadrupolar interactions can be fulfilled by fitting only the variation of the centerband intensities with the relative rotor phase. The advantage of this method is that the centerband usually contains most of the intensity of the central transition which is necessary to obtain the best accuracy of the fitting between simulations and the experimental results. Figure (4.10) shows the parametric plots of the relative phase $\gamma_{r}$ dependence of the centerband intensities with different asymmetry parameters, $\eta$, of the quadrupolar interactions.

The most interesting feature observed from the variations of the sideband intensities with the relative rotor phase is that near the centerband, odd sidebands have inverse symmetry about the relative phase $\gamma_{r}=0$ and $\gamma_{r}=\pi$. Practically, such an inverse symmetry can be used to eliminate the odd sidebands and then to improve the spectral resolution. To fulfil that, the outer rotor has to be synchronized at 

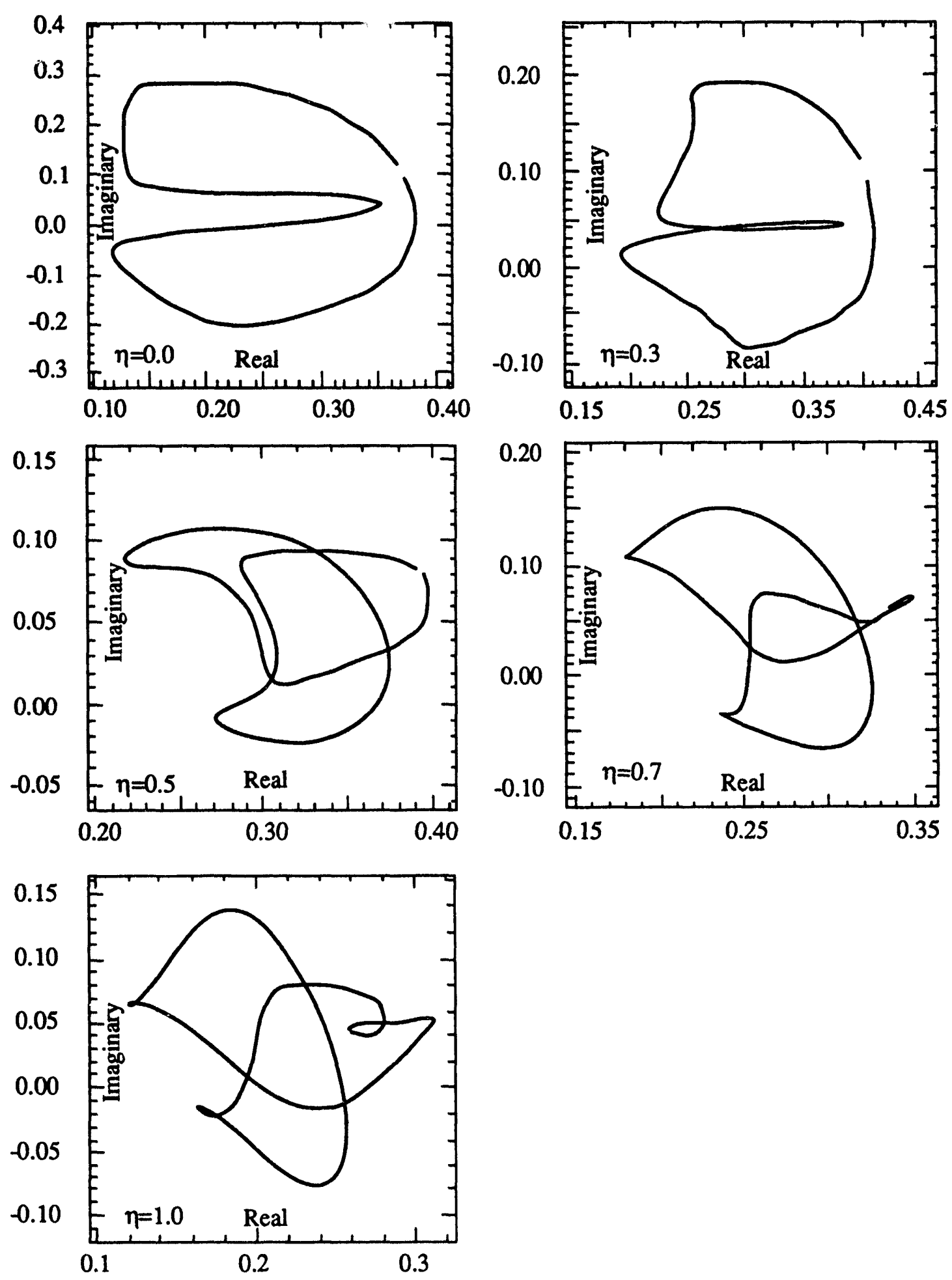

Figure 4.10: Parametric plots of the variation of the DOR centerband intensities as the relative rotor phase $\gamma_{r}$ between the inner and outer rotors changes from $0^{\circ}$ to $360^{\circ}$ with different asymmetry parameters $(\eta)$, where the quadrupolar coupling constant is $\omega_{Q}=433 \mathrm{kHz}$, the Larmor frequency is $\omega_{0}=105.8 \mathrm{M} \mathrm{Hz}$, and the outer rotor spinning speed is $700 \mathrm{~Hz}$ while the inner rotor spins at the rate of $3.3 \mathrm{kHz}$. 
$\gamma_{r}=0^{\circ}$ and $\gamma_{r}=180^{\circ}$. After adding the two spectra together, the odd sidebands near the centerband can be totally suppressed $[112,104]$. Figure (4.11) show the experimental result of sodium oxalate.

In order to see this inverse symmetry from Eq. (4.46), we extend to the ultimate case in which the spinning speed of the inner rotor is much larger than the second order of the quadrupolar interaction. Hence, all terms relative to the $\omega_{r 2}$ in Eq. (4.27) can be ignored, yielding the phase of the magnetization evolved at time $t$ as

$$
\varphi(t)=\sum_{m=1}^{4} B_{m 0}\left[\sin m\left(\gamma_{r}+\omega_{r 1} t\right)-\sin m \gamma_{r}\right]
$$

Now, we can define $\varphi_{e}$ and $\varphi_{o}$ by

$$
\left\{\begin{array}{l}
\varphi_{e}\left(t, \gamma_{r}\right)=\sum_{m=2,4} B_{m 0}\left[\sin m\left(\gamma_{r}+\omega_{r 1} t\right)-\sin m \gamma_{r}\right] \\
\varphi_{o}\left(t, \gamma_{r}\right)=\sum_{m=1,3} B_{m 0}\left[\sin m\left(\gamma_{r}+\omega_{r 1} t\right)-\sin m \gamma_{r}\right] .
\end{array}\right.
$$

Substitute $\gamma_{r}=0^{\circ}$ and $\gamma_{r}=180^{\circ}$ into Eq (4.51), the observing phase can be represented as

$$
\left\{\begin{array}{l}
\varphi\left(t, \gamma_{r}=0^{\circ}\right)=\varphi_{e}\left(t, \gamma_{r}=0^{\circ}\right)+\varphi\left(t, \gamma_{r}=0^{\circ}\right) \\
\varphi\left(t, \gamma_{r}=180^{\circ}\right)=\varphi_{e}\left(t, \gamma_{r}=0^{\circ}\right)-\varphi\left(t, \gamma_{r}=0^{\circ}\right)
\end{array}\right.
$$

If we average the FID signal generated by Eq. (4.52), the total FID for central transition is

$$
\overline{g(t)}=\int_{\Omega} d \Omega \exp ^{-i \varphi_{e}\left(t, \gamma_{r}=0^{\circ}\right)}\left\{e^{-i \varphi_{e}\left(t, \gamma_{r}=0^{\circ}\right)}+e^{i \varphi_{0}\left(t, \gamma_{r}=0^{\circ}\right)}\right\}
$$

Using the property of Bessel function, the FID can be written in a series of Bessel function

$$
\overline{g(t)}=\int_{\Omega} d \Omega \exp ^{-\imath \varphi_{\mathrm{e}}\left(t, \gamma_{\mathrm{r}}=0^{\circ}\right)} \sum_{N, k}\left[1+(-1)^{N}\right] J_{N-3 k}\left(B_{10}\right) J_{k}\left(B_{30}\right) e^{\imath N \omega_{\mathrm{r} 1} t}
$$

In Eq. (4.54), the $\varphi_{e}$ only contributes to even sidebands because the basic harmonic frequency is $2 \omega_{r 1}$. The odd sidebands are then determined by the second part. When $N$ is odd number, the intensities of all odd order harmonics are zero. 
(a)

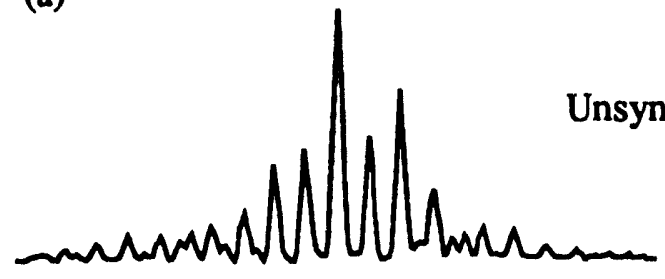

(b)

nized

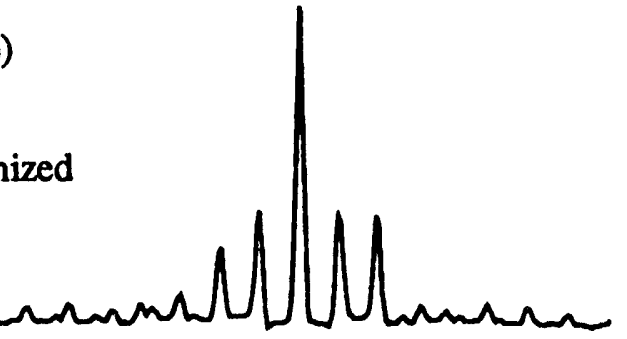

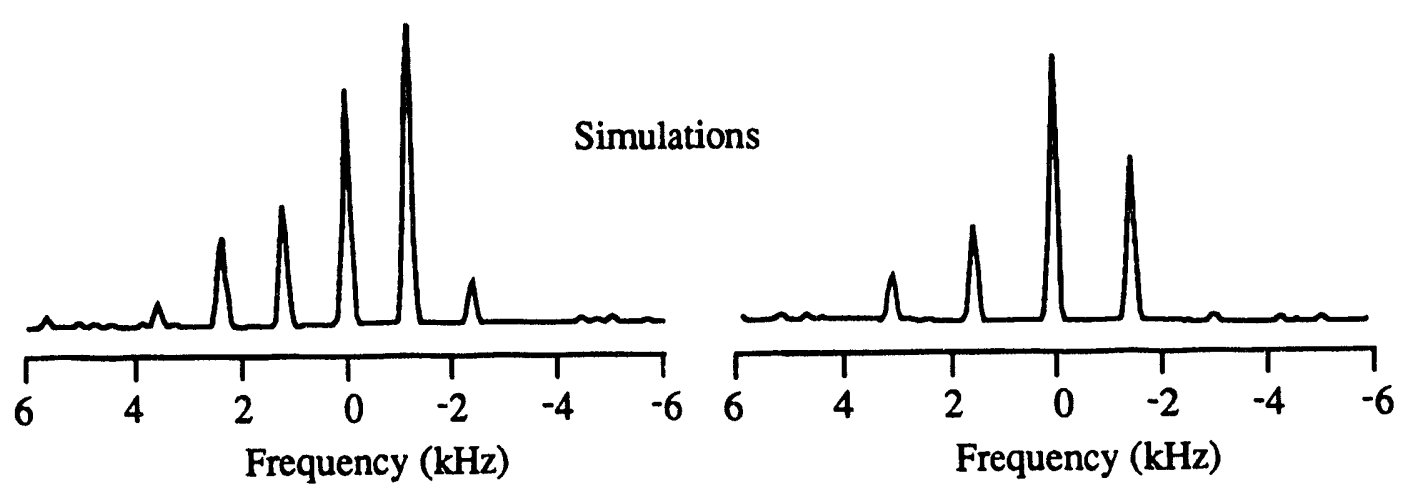

Figure 4.11: Suppression of odd number sidebands by use of the inverse symmetry of the odd number sidebands about the relative phase $\gamma_{r}=0$ and $\gamma_{r}=\pi$. (a) $\omega_{R}=604 \mathrm{~Hz}$, and (b) $\omega_{R}=800 \mathrm{~Hz}$. The computer simulation is shown in the bottom row with $\omega_{Q}=405 \mathrm{kHz}$ and $\eta=0.72$. 


\subsubsection{Conclusions}

Double rotction (DOR) can efficiently suppress the second order broadenings arising from the quadrupolar interactions under the fast spinning speed condition and only if the ratio of the two spinning speeds larger than four. Under slow spinning speed condition, the broadenings still can be narrowed with companion of sidebands. Through the moment analysis of the DOR sidebands, only the second or lower moments are independent of the sample spinning speeds. The dependence of the third moment on the sample spinning speed explains why the envelop of the DOR sidebands does not mimic the $\varepsilon^{+}$atic powder lineshape and changes with the sample spinning speeds. The lineshape characteristic parameter $\mu=M_{4} / m_{2}^{2}$ increases linearly and bilinearly with the sample spinning speeds, The change of the envelop of the DOR sidebands is according to the quasi-supper-Lorentzian. From Bessel function analysis of the DOR sidebands, the variations of the sideband intensities are determined by the sample spinning speeds, anisotropies of the quadrupolar interaction, and the relative rotor phase between the outer and inner rotors. The existence of the inverse symmetry of the odd number sidebands for the relative rotor phase at $\gamma_{r}=0$ and at $\gamma_{r}=\pi$ allows us to completely suppress the odd number sidebands by adding the two spectra and thus to improve the spectral resolution. By the use of average Hamiltonian theory, properties of irreducible tensors, and Bessel functions, the sideband intensities can be exactly evaluated (see Eqs. (4.43), (4.46), and (4.49)). Computer simulations reveal all of the above properties of the sidebands in DOR spectra and results are satisfied with experimental results. Based on these simulations, the anisotropic information of quadrupolar interactions can be extracted.

All of the above results can be applied to any other inhomogeneous interactions. For homogeneous interaction, if $\omega_{r 2}$ is much larger than the amplitude of the interaction, we can average over second rotation first, reorienting spins along the rotation axis. The broadening then becomes approximately inhomogeneous. By applying the 
first rotation without the condition of fast spinning, the broadened line will split to a set of sidebands and the spectral resolution will be much higher than those resulting from the MAS even with the same spinning speed.

\subsection{Sidebands in DAS NMR Experiments}

In the last chapter we described the general principles of the dynamic angle spinning (DAS) based on the assumption that the spinning speed of the sample during the experiment is much larger than the amplitude of the first order average Hamiltonian with the quadrupolar interactions of half integer spin nuclei. In practice, owing to the mechanical properties of the spinning system, the spinning speed usually cannot satisfy the fast spinning condition. Under this circumstance, the time modulated spin Hamiltonian will develop a train of sidebands in DAS spectra like those in MAS spectra. This is because the first order average Hamiltonian of the quadrupolar interaction is still inhomogeneous. In this section we present the general treatment of the DAS sidebands based on the moment analysis and Bessel function analysis methods described in last section. Using these methods, the sideband intensities are numerically evaluated. The results show that the intensities of the DAS sidebands are dependent not only on the spinning speed and the principal values of the quadrupolar tensors, but also on the relative rotor phase and the time ratio between the first and the second evolutions. Both the intensities and the phases of the sidebands vary with the relative phase, but no inverse symmetry like that in DOR has be found in the variations of the sideband intensities with the relative phase in DAS spectra. The dependence on the time ratio between the two evolutions in the DAS experiments results complicated DAS sideband structure. Additional sidebands between one rotational cycle appears, and the number of the additional sidebands in one rotation cycle is determined by the time ratio, $k_{t}=\frac{t_{1}}{t_{2}}$. For example, in the case of $k=1$, there are two sidebands: one is at $\omega_{r} / 2$ and the other is at $\omega_{r}$, where $\omega_{r}$ is 
the sample spinning speed.

\subsubsection{FID Signal of DAS NMR}

In DAS experiments (see Figure (3.15)), the sample rotates around an axis inclined at the angle of $\theta_{1}$ with respect to the external magnetic field $H_{0}$ during evolution time $t_{1}$, and at another angle of $\theta_{2}$ during evolution time $t_{2}$. The time dependence of the central transition frequencies with quadrupolar interactions in a half integer spin nuclear system is given by Eq.(3.25), or

$$
\omega_{\frac{1}{2} \leftrightarrow-\frac{1}{2}}^{(2)}\left[\Omega_{r}(t)\right]=\sum_{l=0,2,4} \sum_{m} C_{l} \mathcal{D}_{m, 0}^{(l)}\left[\Omega_{r}(t)\right] A_{l, m},
$$

where $C_{l}$ is given by Eq.(3.26). At the end of the second evolution time of $t_{2}$, the total evolved phase of the magnetization under the first order average Hamiltonian with the quadrupolar interaction is

$$
\varphi\left(t_{1}, t_{2}\right)=\int_{0}^{t_{1}} \omega_{\frac{1}{2} \leftrightarrow-\frac{1}{2}}^{(2)}\left[\Omega_{r_{1}}(t)\right] d t+\int_{t_{1}}^{t_{1}+t_{2}} \omega_{\frac{1}{2} \leftrightarrow-\frac{1}{2}}^{(2)}\left[\Omega_{r_{2}}(t)\right] d t .
$$

Two sets of Euler angles are defined by

$$
\left\{\begin{array}{l}
\Omega_{r_{1}}(t)=\Omega\left(\omega_{r} t+\phi_{1}, \theta_{1}, \gamma_{1}\right) \\
\Omega_{r_{2}}(t)=\Omega\left(\omega_{r} t+\phi_{2}, \theta_{2}, \gamma_{2}\right)
\end{array}\right.
$$

where we have assumed that the spinning speeds during different evolution times are same, $\phi_{1}$ and $\phi_{2}$ are the initial phases of the azimuth angle at the beginning of the two evolution times, and $\gamma_{1}$ and $\gamma_{2}$ are the initial phases of the rotation axis relevant to the laboratory frame. Owing to the cylindrical symmetry of the magnetization around the external field $H_{0}, \gamma_{1}$ and $\gamma_{2}$ may be set to zero. If we acquire the data at the DAS echo tops, $t_{2}=k_{t} t_{1}$ ( $k_{t}$ is same as $k$ in chapter 3 ) where $\frac{1}{5} \leq k_{t} \leq 5$ from Eq.(3.30). Thus using the representation of the Wigner rotation matrices, inserting Eqs.(4.55) and (4.57) into Eq.(4.56) yields the FID signal in the DAS experiment.

$$
g(t)=\exp \left\{\sum_{m \neq 0} e^{-\imath m \alpha}\left[B_{m}\left(\theta_{2}\right) e^{-\imath m\left(\phi+\omega_{r} t\right)} \psi\left(-k_{t} t\right)-B_{m}\left(\theta_{1}\right) \psi(t)\right]\right\}
$$


where

$$
\begin{aligned}
B_{m}(\theta) & =\sum_{l=2,4} \frac{C_{l} A_{l, m}}{m \omega_{r}} d_{m, 0}^{(l)}(\theta), \quad|m| \leq l \\
\psi(t) & =1-e^{-i \frac{m \omega_{r} t}{1+k_{l}}}
\end{aligned}
$$

and $t=t_{1}+t_{2}$. In Eq.(4.58) we have assumed that the magnetization is on resonance, the $m=0$ term is zero by the requirement of DAS condition and thus should not be included. The powder averaged FID signal may be obtained by inserting Eq.(4.58) into Eq.(4.30). The initial phase $\phi_{1}$ becomes unimportant and therefore is set to zero and $\phi_{2}=\phi$.

\subsubsection{Moment Analysis of DAS FID signal}

The moment analysis method has been described in the last section. After inserting Eq (4.58) into Eq.(4.30) and then applying Eq.(4.33), the expressions of the moments of DAS NMR spectra are same as those of DOR spectra given in Eqs.(4.35). Of course, the coefficients of the scalar operators are different, given by

$$
\begin{aligned}
a_{k}\left[p_{k-3}(L) p_{k}(l)\right] & =\sum_{p_{k-1}(m)} d_{k}\left[p_{k-3}(L) p_{k}(l) p_{k-1}(m), 2\right] \\
a_{3}\left[p_{2}(l)\right] & =\sum_{p_{1}(m)} m_{1} \omega_{r} d_{k}\left[p_{2}(l) p_{1}(m), 3\right] \\
a_{4}\left[p_{3}(l)\right] & =\sum_{p_{2}(m)}\left(m_{1}+m_{2}\right) \omega_{r} d_{k}\left[p_{3}(l) p_{2}(m), 3\right] \\
a_{4}\left[p_{2}(l)\right] & =\sum_{p_{1}(m)} m_{1} \omega_{r} d_{k}\left[p_{2}(l) p_{1}(m), 4\right] \\
d_{k}\left[p_{k-3}(L) p_{k}(l) p_{k-1}(m), n\right] & =C_{l_{1}} \cdots C_{l_{k}} P_{C G}\left[p_{k-3}(L) p_{k}(l) p_{k-1}(m)\right] \\
& \times E_{l_{1}, m_{1}}^{(2)} \cdots E_{l_{k-1}, m_{k-1}}^{(2)} E_{l_{k},-\sum_{i=1}^{(n)} m_{i}}^{k-1}
\end{aligned}
$$

where

$$
E_{l, m}^{(n)}=\frac{1}{\left(1+k_{t}\right)^{n-1}}\left[\left(2^{n-1}-1\right) k_{t} d_{m, 0}^{(l)}\left(\theta_{2}\right) e^{-\imath m \phi}+d_{m, 0}^{(l)}\left(\theta_{1}\right)\right]
$$

and $\rho_{00}(k)$ is the zero rank tensor arising from the product of $k$ second order quadrupolar coupling tensors, defined by Eq.(4.36). The anisotropic parameters $\delta$ and $\eta$ of the 
quadrupolar interaction are encoded in the zero rank tensors which can be explicitly seen in Eq.(4.38). From an experimental spectrum under DAS, the second and third moments may be calculated by using Eq.(4.31) and Eq.(4.60), $\delta$ and $\eta$ may then be extracted in a very manner if we know the initial phase of the ration at the beginning of the second evolution time of $t_{2}$. If the DAS experiment is performed without synchronization of the sample rotation, the average over the initial phase $\phi$ has to be implemented in Eq.(4.60) in order to obtain correct moments.

The fourth and higher order moments do not contain any new information which is not already available from the second and third moments. In the moments of the MAS spectra, both the second and third moments are independent of the spinning speed $\omega_{r}$. However in the moments of the DAS FID signal only the second moment is motion independent and the third and higher moments increases with the spinning speed $\omega_{r}$. This means that the shape of the envelop of the DAS sidebands will change with $\omega_{r}$ which is quite different from the MAS case in which only the halfwidth of the envelop of the MAS sidebands decreases as increasing $\omega_{r}$. In addition, the parameter $\mu=M_{4} / M_{2}^{2}$ increases with both $\omega_{r}$ and $\omega_{r}^{2}$ leading to quasi-super-Lorentzian lines of the envelop of the DAS sidebands.

\subsubsection{Bessel Analysis of DAS Sidebands}

Bessel function analysis method of the sidebands also had been introduced in the last section. From the properties of Bessel functions $J_{k}(z)$ given by Eqs. (4.42) to (4.44), FID signal can be expressed as

$$
\begin{aligned}
g(t) & =\left\{\prod_{m \neq 0} \sum_{i_{m}} \sum_{j_{m}} \sum_{k_{m}} \sum_{l_{m}} \mathcal{J}_{i_{m}}\left[B_{m}\left(\theta_{2}\right)\right] \mathcal{J}_{j_{m}}\left[-B_{m}\left(\theta_{2}\right)\right] \mathcal{J}_{k_{m}}\left[B_{m}\left(\theta_{1}\right)\right] \mathcal{J}_{l_{m}}\left[-B_{m}\left(\theta_{1}\right)\right]\right\} \\
& \times \exp \left\{-\imath\left[N_{\alpha} \alpha+N_{\phi} \phi+\left(N_{r_{1}}+\frac{1}{1+k_{t}} N_{r_{2}}\right) \omega_{r} t\right]\right\}
\end{aligned}
$$

where

$$
N_{\alpha}=\sum_{m=-4}^{4} m\left(i_{m}+j_{m}+k_{m}+l_{m}\right)
$$




$$
\begin{aligned}
& N_{\phi}=\sum_{m=-4}^{4} m\left(i_{m}+j_{m}\right) \\
& N_{r_{1}}=\sum_{m=-4}^{4} m i_{m} \\
& N_{r_{2}}=\sum_{m=-4}^{4} m\left(k_{m}+j_{m}\right)
\end{aligned}
$$

After averaging Eq.(4.62) over $\alpha$, the FID signal becomes

$$
\overline{g(t)}=\sum_{N_{\phi}} g_{N_{\phi}}\left(\theta_{1}, t\right) g_{-N_{\phi}}\left(\theta_{2}, t\right) e^{-\imath N_{\phi} \phi}
$$

where

$$
g_{N_{\phi}}(\theta, t)=\prod_{m \neq 0} \sum_{i_{m}} \sum_{j_{m}} \mathcal{J}_{i_{m}}\left[B_{m}(\theta)\right] \mathcal{J}_{j_{m}}\left[-B_{m}(\theta)\right] e^{-\imath N_{r} \omega_{r} t}
$$

and

$$
N_{r}= \begin{cases}\frac{1}{1+k_{t}} \sum_{m} m i_{m}=\frac{1}{1+k_{t}}\left[N_{\phi}-\sum_{m} m j_{m}\right] & \text { for } \theta=\theta_{1} \\ \sum_{m} m\left(i_{m}+\frac{1}{1+k_{t}} j_{m}\right)=\frac{1}{1+k_{t}}\left[k_{t} \sum_{m} m i_{m}+N_{\phi}\right] & \text { for } \theta=\theta_{2} .\end{cases}
$$

Using Eq.(4.43), the FID signal becomes

$$
\overline{g(t)}=\sum_{N_{\phi}} \sum_{N_{1}, N_{2}} I_{N_{1}}^{*}\left(\theta_{1}\right) I_{N_{1}-N_{\phi}}\left(\theta_{1}\right) I_{N_{2}}\left(\theta_{2}\right) I_{N_{2}-N_{\phi}}^{*}\left(\theta_{2}\right) e^{-\mathfrak{z}\left[N_{\phi} \phi+\left(N_{1} \omega_{r_{1}}+N_{2} \omega_{r_{2}}\right) t\right]}
$$

where

$$
I_{N}(\theta)=\frac{1}{2 \pi} \int_{0}^{2 \pi} d \alpha e^{z\left(N \alpha-\sum_{m \neq 0} B_{m}(\theta) e^{-\imath m \alpha}\right)}
$$

and

$$
\omega_{r_{1}}=\frac{1}{1+k_{t}} \omega_{r}, \quad \omega_{r_{2}}=\frac{k_{t}}{1+k_{t}} \omega_{r} .
$$

After averaging Eq.(4.67) over a powder sample, the total FID signal is given by

$$
G(t)=\sum_{N_{1}, N_{2}} I_{N_{1}, N_{2}} e^{-\imath\left(N_{1} \omega_{r_{1}} t+N_{2} \omega_{r_{2}}\right) t}
$$

where

$$
I_{N_{1}, N_{2}}=\frac{1}{4 \pi} \int_{0}^{2 \pi} \int_{0}^{\pi} \sin \beta d \beta d \gamma \sum_{N_{\phi}} I_{N_{1}-N_{\phi}}\left(\theta_{1}\right) I_{N_{1}}^{*}\left(\theta_{1}\right) I_{N_{2}}\left(\theta_{2}\right) I_{N_{2}-N_{\phi}}^{*}\left(\theta_{2}\right) e^{-\imath N_{\phi} \phi} .
$$


(a) Real
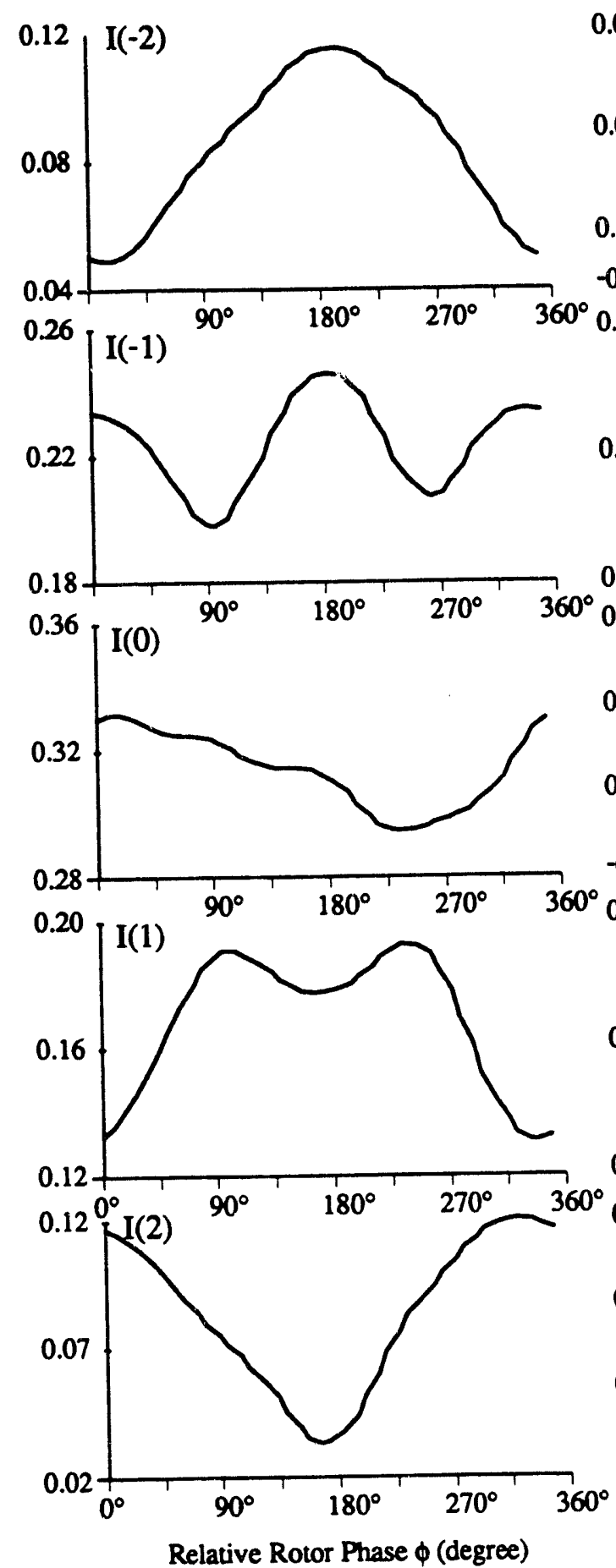

(b) Imaginary
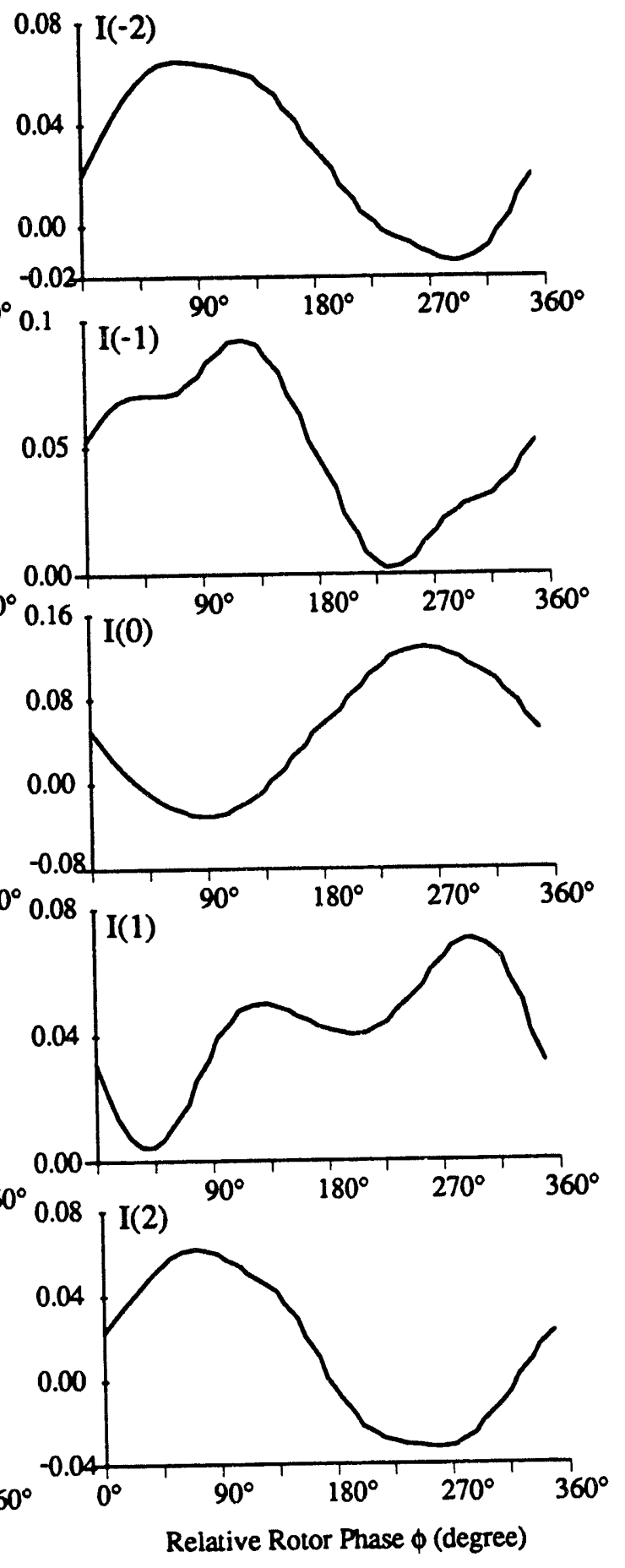

Figure 4.12: Variations of the simulated DAS sideband intensities, $I_{N_{1}, N_{2}}$, as the relative phase, $\phi$ changes for the case that $k=1, \theta_{1}=37.38^{\circ}$ and $\theta_{2}=79.19^{\circ}$. The quadrupolar coupling constant is $\omega_{Q}=405 \mathrm{kHz}$, its asymmetry parameter is $\eta=0.72$, from sodium oxalate; the sample spinning speed is $\omega_{r}=3 \mathrm{KHz}$, and the Larmor frequency is $\omega_{0}=105.8 \mathrm{MHz}$. 
What we can conclude from Eq.(4.64) is that the overall sideband pattern under DAS is sum over a set of the convolutions of two individual sets of sidebands due to the evolution during times $t_{1}$ and $t_{2}$ respectively. After $g_{N_{\phi}}(\theta, t)$ is expanded into a Fourier series, the coefficients (intensities) of each individual harmonic is dependent on all parameters during the whole evolution. The effect of the relative phase $\phi$ is also dependent on the parameters and in general cannot be used to edit the spectra obtained with different relative phase such that some of the sidebands can be suppressed. This feature can also be seen from Eq.(4.59), the symmetry of the coefficients, $B_{m}$ is dependent on the symmetry of $A_{m}$, unlike in the DOR case which $A_{l, m}$ is truncated by the inner rotor spinning. Thus the accumulated phase of the magnetization given by Eq.(4.58) cannot be represented as the form of Eq.(4.51) when $\phi=0$ and $\phi=\pi$. Furthermore, from the numerical simualtions of the variations of the intensities and the phases of the DAS sidebands as the relative phase $\phi$ changes from $0^{\circ}$ to $360^{\circ}$ shown in Figure (4.12), the real components of the DAS sidebands are always positive for the case of $k_{t}=1$, corresponding to $\theta_{1}=37.38^{\circ}$ and $\theta_{2}=79.19^{\circ}$, and the spinning speed is $\omega_{r}=2 k H z$ or higher, where we assume that the quadrupolar coupling constant is $\omega_{Q}=405 \mathrm{kHz}$, the asymmetry parameter is $\eta=0.72$, and the Larmor frequency of the nuclear species involved is $105.8 \mathrm{MHz}$.

Experimentally if the rotor is not synchronized during spinning the relative phase is a random variable for different acquisitions. This is equivalent to taking an average over the relative initial phase, resulting in $N_{\phi}=0$. Using Eq.(4.44), the sideband intensity becomes

$$
\overline{I_{N_{1}, N_{2}}}=\frac{1}{4 \pi} \int_{0}^{2 \pi} \int_{0}^{\pi} \sin \beta d \beta d \gamma\left|I_{N_{1}}\left(\theta_{1}\right)\right|^{2}\left|I_{N_{2}}\left(\theta_{2}\right)\right|^{2} .
$$

Using Eq.(4.72), we have evaluated the sideband intensities varying with the sample spinning speed, $\omega_{r}$, shown in Figure (4.13). In the simulations, we used composite ten-point Gaussian (Gauss-Legendre) quadruture to integrate over $\alpha, \beta, \gamma$. The pa- 

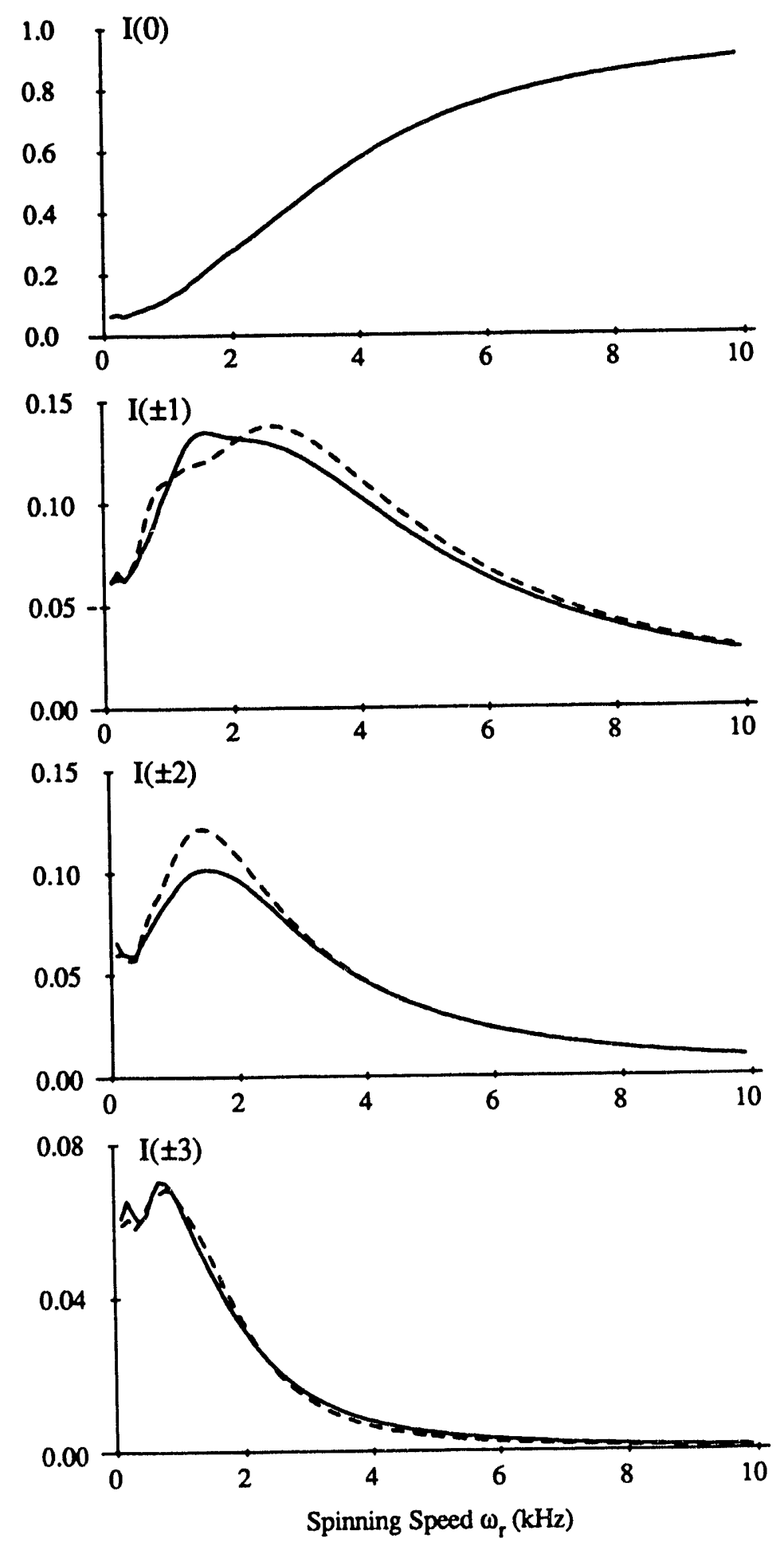

Figure 4.13: Variations of the simulated DAS sideband intensities, $I_{N_{1}, N_{2}}$, with the sample spinning speed for the case that $k=1$, corresponding to $\theta_{1}=37.38^{\circ}$ and $\theta_{2}=79.19^{\circ}$, and $N_{2}=0, N_{1}=0, \pm 1, \pm 2$, where the quadrupolar coupling constant of the sodium-23 nucleus $\omega_{Q}=405 \mathrm{kHz}$, its asymmetry parameter is $\eta=0.72$, and the Larmor frequency is $\omega_{0}=105.8 \mathrm{M} \mathrm{Hz}$. 
rameters used in the simulations are $\omega_{Q}=405 \mathrm{kHz}, \eta=0.72$, and $\omega_{0}=105.8 \mathrm{M} \mathrm{Hz}$. As can be seen from the figure, the sideband intensities around the centerband distribute more symmetric than those in MAS. This feature leads to the sideband intensities are not very sensitive to the asymmetry parameter, $\eta$.

The structure of the sidebands in DAS NMR spectra can be interpreted as follows. If we assume that $k_{t}=\frac{k_{1}}{k_{2}}$ where $k_{1}$ and $k_{2}$ are two integers, the maximum number of sidebands is $k_{1}+k_{2}$ in one rotor cycle $\omega_{r}$. Of course the intensities of the sidebands are different. In general those corresponding to small $N_{1}$ and $N_{2}$ have larger intensities and therefore there are three main sidebands in one cycle whose frequencies are $\omega_{r_{1}}$, $\omega_{r_{2}}$, and $\omega_{r}$ respectively. In the case of $k_{t}=1$, two sidebands in the middle will overlap together and the whole pattern is uniquely determined by the frequency $\omega_{r} / 2$. Figure (4.14) shows the simulated spectra of polycrystalline sodium oxalate using same parameters as in Figure (3.29). The other simplest solution is for $k_{t}=5$. In this case one of the rotation axis is aligned on the external magnetic field $H_{0}$, that is, $\theta_{1}=0$, and then the coefficients of $B_{m}(0)=0$ when $m \neq 0$. In Eq.(4.72) the number of summations furthermore reduces to one. The frequency difference between two nearest sidebands is $\omega_{r_{2}}$. In other words, the maximum spectral resolution can be obtained under this situation.

\subsection{Conclusion}

In this section we described the general theories of the DAS sidebands based on the moment analysis and Bessel function analysis methods. From the moment analysis of the DAS NMR spectra, the third and higher moments increase with $\omega_{r}$, and the envelop of the DAS sidebands changes according to a quasi-super-Lorentzian lineshape as the sample spinning speed increases. Using Bessel function analysis of the DAS sidebands, the structure of the sidebands are a sum over a set of convolutions of two individual sets of sidebands involved in the evolution times of $t_{1}$ and $t_{2}$ respectively, 


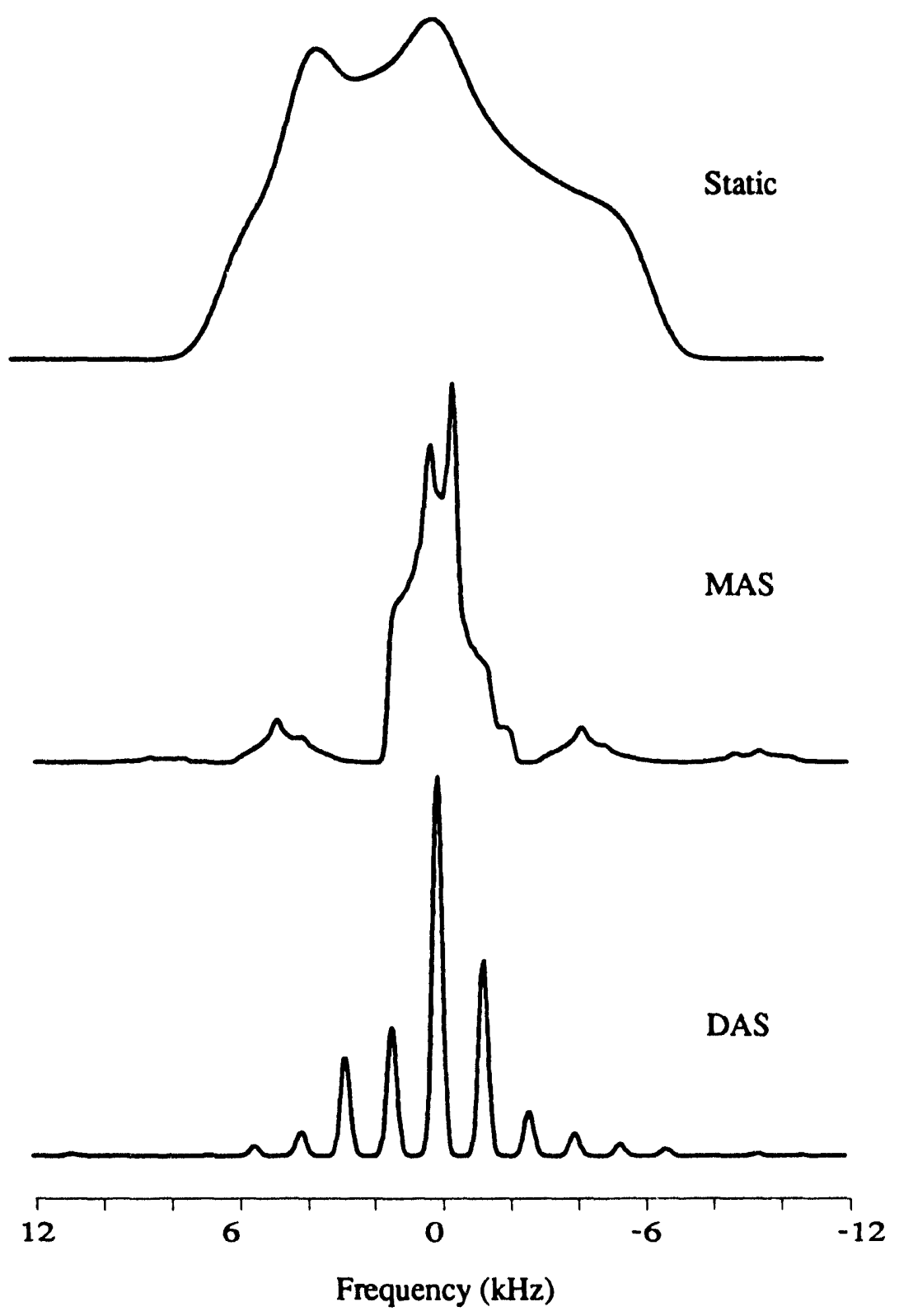

Figure 4.14: Simulated DAS spectra using same parameters in DAS experiment of sodium oxalate, that is, the quadrupolar coupling constant of the sodium-23 nucleus $\omega_{Q}=405 \mathrm{kHz}$, its asymmetry parameter is $\eta=0.72$, and the Larmor frequency is $\omega_{0}=105.8 M H z$. (a) Static powder lineshape; (b) residual lineshape after MAS; (c) DAS spectrum obtained with $\omega_{r}=3.36 \mathrm{kHz}$ 
and the sideband intensities are numerically evaluated. The results show that the intensities of the DAS sidebands are dependent not only on the spinning speed and the principal values of the quadrupolar tensors, but also on the relative phase and the time ratio between the first and the second evolutions. Both the intensities and the phases of the sidebands vary with the relative phase, but no inverse symmetry like that in DOR has be found in the variations of the sideband intensities with the relative phase in DAS NMR spectra. After averaging the sideband intensities over the relative phase, the sideband intensities become more or less symmetric around the centerband, especially when the sample spinning speed is in the fast region, and thus, they not very sensitive to the asymmetry parameter, $\eta$. The dependence on the time ratio between the two evolutions in the DAS experiments results in that the structure of the DAS sidebands becomes very complicated. Additional sidebands between one rotation cycle appears, and the number of the additional sidebands in one rotation cycle is $k_{1}+k_{2}$, assuming the time ratio is $k_{t}=\frac{k_{1}}{k_{2}}$, where $k_{1}$ are $k_{2}$ are integers. For example, in the case of $k_{t}=1$, leading to $k_{1}=k_{2}=1$, there are three sidenabds: two are at $\omega_{r} / 2$ and the other is at $\omega_{r}$, where $\omega_{r}$ is the sample spinning speed. We also show the possibility of the extraction of the principal values of the quadrupolar interactions by simulating the sideband intensities. 


\section{Chapter 5}

\section{Motions in Solid $C_{60}$}

\subsection{Introduction}

A sixty carbon $\left(C_{60}\right)$ molecule having a molecular structure with icosahedral symmetry, was first proposed theoretically by Kroto and his coworkers in the early $1980 \mathrm{~s}[125,124]$, and has been named buckminsterfullerene. Subsequent to the early mass spectroscopy experiments, some micro-quantities of this molecule was obtained in cluster beam experiments by use of a polarized laser to vaporize the graphite [126]. More recently, macroscopic amounts of $C_{60}$ molecules [127] have been synthesized, stimulating intense interest and activity. A number of spectroscopic studies of this molecule have been carried out confirming to the icosahedral symmetry of $C_{60}$ molecules $[135,136]$. Further studies reveal that materials arising from the $C_{60}$ molecules intercalated with alkali metal atoms to form the 'fullerides' $A_{x} C_{60}$ become superconductors [128] below the critical temperatures $T_{c}$. The critical temperatures $T_{c}$ depend on the alkali metal atoms used in the intercalation, and for $A=K[130,129] T_{c}=18 K$, while $T_{c}=28 K$ when $A=R b[131]$.

NMR spectroscopy experiments of $C_{60}$ molecules were performed first by Tycko and his coworkers[137] as well as Yannoni and his coworkers $[139,140]$. The spectra obtained from their variable temperature solid-state NMR experiments showed some interesting lineshapes. These lineshapes consist of a sharp peak at the center indicating the isotropic chemical shift, and a CSA-type powder pattern. Based on 
these spetra, they concluded that the sharp peaks are due to some residual carbon clusters whose motions are relatively fast when compared to the $C_{60}$ molecules. In this chapter, we assume that the motions of $C_{60}$ molecules in the solid state consist of icosahedral jumps. By assuming this model, we have been able to simulate the powder lineshapes measured by Tycko and Yannoni et al. The results show that the samples of solid $C_{60}$ are indeed not homogenous, resulting the sharp peak which indicates that the carbon clusters have different motions from the $C_{60}$ molecules.

We will use a chemical exchange model to study the jump motions (molecular reorientations) in $C_{60}$ molecules. The random jump motion of the $C_{60}$ molecules is physically different from a chemical exchange process. Chemical exchange is defined by migration of atoms or groups of atoms from one molecule to another or from one part of a molecule to another which can be distinguished by the different resonance frequencies. Such exchanges can happen in both inter- and intra-molecule. In the $C_{60}$ molecules, atoms are relatively fixed and cannot move around physically. Since the molecules themselves have very high symmetry, there are sitting in potential barriers which are determined by the crystal symmetry. The motions of the $C_{60}$ molecules from one configuration to another have to be very quick, and look like sudden jumps from one configuration to another. Thus, the jumps of the atoms in a molecule is via the jumps of the molecule itself. For this reason, the chemical exchange theory will be reviewed in the first few sections. However, we do not just restrict ourself to the literature, but will emphasize the lineshape changes for different cases based on our own simulation results.

\subsection{Theory of Chemical Exchange}

As a simple example, let us first consider a chemical exchange process of two-site jumps. We assume that the jump from one frequency to another is a stationary Markov process[5, 142]. From this assumption, one can conclude that the jumping 
rate from one site to another is time independent. Then from the modified Bloch equation, the evolution of the magnetization at two sites is determined by

$$
\begin{aligned}
& \frac{d}{d t} M_{1}(t)=w \omega_{1} M_{1}(t)+\kappa\left[M_{2}(t)-M_{1}(t)\right] \\
& \frac{d}{d t} M_{2}(t)=w \omega_{2} M_{2}(t)-\kappa\left[M_{2}(t)-M_{1}(t)\right]
\end{aligned}
$$

where $M_{1}$ and $M_{2}$ are the magnetizations, $\omega_{1}$ and $\omega_{2}$ are the frequencies at site 1 and 2 respectively, and $\kappa$ is the jumping rate from site 1 to site 2 or vice visa. By use of the standard method for solving a linear, first order differential equations. we find the solution of Eq. (5.1) to be

$$
\begin{aligned}
& M_{1}(t)=c_{1+} e^{\lambda_{+} t}+c_{1-} e^{\lambda-t} \\
& M_{2}(t)=c_{2+} e^{\lambda_{+} t}+c_{2-} e^{\lambda_{-} t}
\end{aligned}
$$

where

$$
\lambda_{ \pm}=\frac{1}{2}\left\{\imath\left(\omega_{1}+\omega_{2}\right)-2 \kappa \pm \sqrt{4 \kappa^{2}-\left(\omega_{1}-\omega_{2}\right)^{2}}\right\},
$$

and

$$
c_{i \pm}= \pm \frac{\left(\lambda_{ \pm}-\omega_{i}\right) M_{i}(0)+\kappa\left[M_{1}(0)+M_{2}(0)\right]}{\lambda_{+}-\lambda_{-}}
$$

for $i=1,2$ and $M_{i}(0)$ is the initial magnitization of the $i$-th site.

As can be seen from Eq.(5.3), the frequency difference of two sites decreases, and the linewidths of two separated peaks are uniquely determined by the exchange rate $\kappa$ as it increases, but satisfies the condition that $\kappa<\left(\omega_{1}-\omega_{2}\right) / 2$. On the other hand, when $\kappa>\left(\omega_{1}-\omega_{2}\right) / 2$, the two peaks coalesce, but the linewidth decreases according to two exponential functions as the exchange increases. The width of the component corresponding to eigenvalue $\lambda_{+}$decreases to zero as $\kappa$ increases, and the other component becomes very broad (see Fig. (5.1)) while its amplitude tends to zero. After Fourier transforming Eq.(5.2), the lineshape of the spectrum can be represented as

$$
M(\omega)=\frac{1}{2} \frac{\kappa\left(\omega_{1}-\omega_{2}\right)^{2}}{\left[\left(\omega-\omega_{1}\right)\left(\omega-\omega_{2}\right)\right]^{2}+\kappa^{2}\left[2 \omega-\left(\omega_{1}-\omega_{2}\right)\right]^{2}} .
$$


(a)

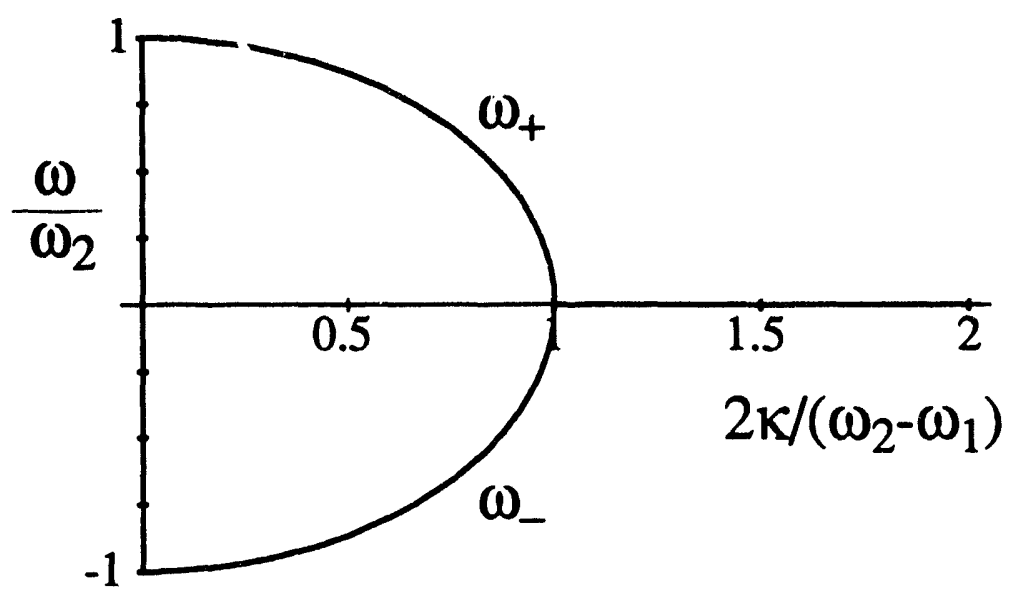

(b)

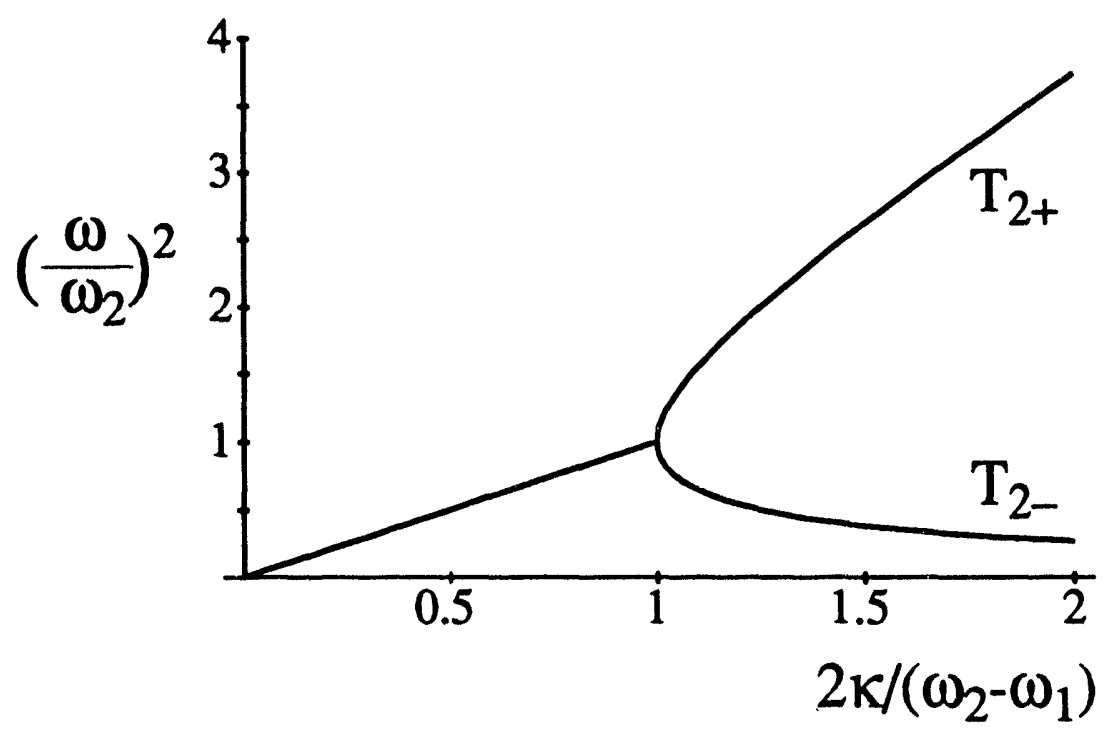

Figure 5.1: Dependences of the resonance frequencies and the linewidths with the exchange rate, $\kappa$, in two-site jump model: (a) frequency dependence; (b) linewidth dependence 
Fig.(5.2) shows variation of the lineshapes of the two-site jump model with exchange rate, $\kappa$.

In order to generalize chemical exchange theory to $n$-site jumps, we introduce the exchange rate matrix $\hat{\pi}$ whose elements $\kappa_{i j}$ represent the exchange rate from site $i$ to $j$. By the population conservation, we have

$$
\sum_{i, j} \kappa_{i j}=0 .
$$

Similarly, we define the magnetization vector as $\mathbf{M}=\left(M_{1}, M_{2}, \cdots, M_{n}\right)$, and the frequency matrix with diagonal elements $\omega=\left(\omega_{1}, \omega_{2}, \cdots, \omega_{n}\right)$. Now the dynamical equation of magnetization for $n$-site jumps can be written as

$$
\frac{d}{d t} \mathbf{M}(t)=[\imath \hat{\boldsymbol{\omega}}+\hat{\boldsymbol{\pi}}] \cdot \mathbf{M}(t)
$$

The formal solution of Eq.(5.7) is

$$
\mathbf{M}(t)=e^{\imath(\hat{\boldsymbol{\omega}}+\hat{\pi}) t} \mathbf{M}(0),
$$

or in frequency domain,

$$
M(\omega)=\mathbf{1} \cdot \hat{\mathbf{A}}^{-1}(\omega) \cdot \mathbf{M}(0)
$$

where $\hat{\mathbf{A}}(\omega)=\omega \hat{\mathbf{1}}-\imath \hat{\boldsymbol{\omega}}+\hat{\boldsymbol{\pi}}, \mathbf{1}=(1,1, \cdots, 1)$, and $\hat{\mathbf{1}}$ is a unit matrix.

For a general chemical exchange process, it is very difficult to solve Eq.(5.8) or (5.9). The general solution also gives very little physical insight. For most cases, one only considers two extreme situations: (i) Nearest neighbor exchange, also called the weak collision approximation, in which $\kappa_{i, i+1}=\kappa_{i, i-1}=\kappa$ and $\kappa_{i, i}=-2$. (ii) All site exchange, the strong collision approximation, with equal rates for all exchanges.

The analytical solution for the strong collision limit was first cbtained by Alexander and his coworkers[144] in 1977 by use of representation theory of the symmetry groups. Later, Wemmer et al. $[145,146]$ applied matrix manipulation to invert the 


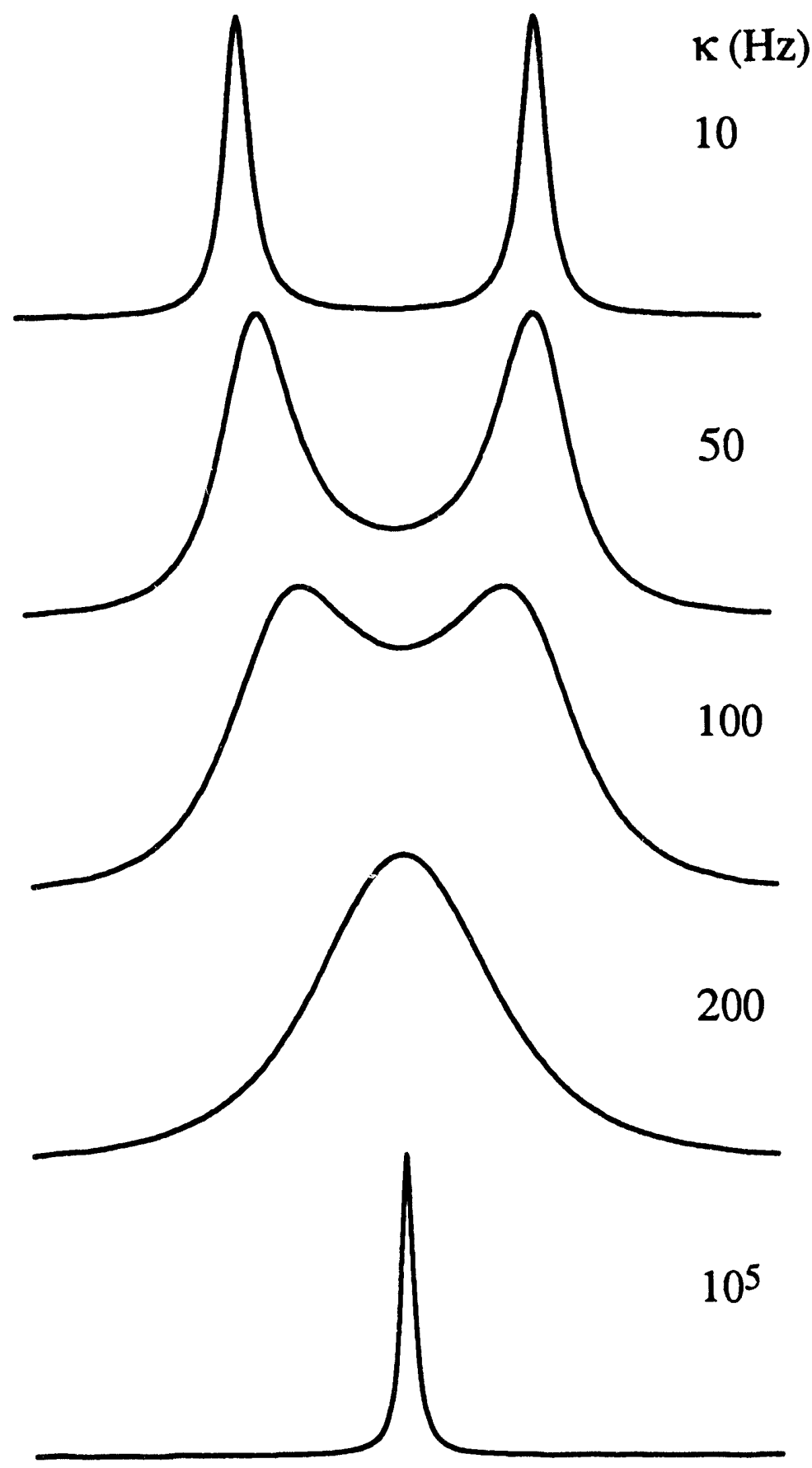

Figure 5.2: Variation of the lineshapes in two-site jumps with different jumping rates, $\kappa$, where $\omega_{1}=-\omega_{2}=200 \mathrm{~Hz}$, and $\kappa$ is in unit of Hertz. 
matrix $\hat{\mathbf{A}}$. Based on this matrix manipulation method, we solve the chemical exchange process in strong collision limit following the convention given by Mehring [19].

According to the strong collision limit, the exchange rate matrix $\hat{\pi}$ has the form

$$
\hat{\boldsymbol{\pi}}=\kappa\left[\begin{array}{ccccc}
-(N-1) & 1 & 1 & \cdots & 1 \\
1 & -(N-1) & 1 & \cdots & 1 \\
\vdots & \vdots & \vdots & \vdots & \vdots \\
1 & \cdots & \cdots & \cdots & -(N-1)
\end{array}\right]=-N \kappa \hat{\mathbf{1}}+\kappa \hat{\mathbf{I}}
$$

where $\hat{\mathbf{I}}$ consists of only ones. For convenience, we define a diagonal matrix $\hat{\mathbf{B}}$ as

$$
\hat{\mathbf{B}}=(\imath \omega+N \kappa) \hat{\mathbf{1}}-\imath \hat{\boldsymbol{\omega}}
$$

and a regular matrix $\hat{\mathbf{C}}$ as

$$
\hat{\mathbf{C}}=-\kappa \hat{\mathbf{I}} \text {. }
$$

Then the matrix $\hat{\mathbf{A}}$ in the formal solution, Eq.(5.9), of chemical exchange becomes

$$
\hat{\mathbf{A}}=\hat{\mathbf{B}}+\hat{\mathbf{C}}
$$

In order to calculate the inverse matrix of $\hat{\mathbf{A}}$, we expand $\hat{\mathbf{A}}^{-1}$ into Taylor series

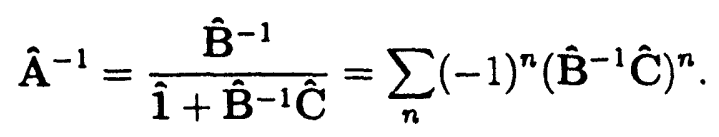

Now we realize that the matrices $\hat{\mathbf{B}}$ and $\hat{\mathbf{C}}$ have the property,

$$
\hat{\mathbf{C}} \hat{\mathbf{B}}^{-1} \hat{\mathbf{C}}=-\kappa L \hat{\mathbf{C}}
$$

where

$$
L=\sum_{j} \frac{1}{2\left(\omega-\omega_{j}\right)+1 / T_{2 j}+N \kappa} .
$$

Insert Eqs.(5.15) and (5.16) into Eq.(5.14), and we obtain

$$
\hat{\mathbf{A}}^{-1}=\hat{\mathbf{B}}^{-1}\left[\hat{\mathbf{1}}+\frac{\hat{\mathbf{C}} \hat{\mathbf{B}}^{-1}}{1-\kappa L}\right] \text {. }
$$


Finally, if we assume that the magnetization at each site is equal, that is $b f M(0)=$ $\frac{1}{N}(1, \cdots, 1)$, the lineshape after chemical exchange can be written as

$$
M(\omega)=1 \cdot \hat{\mathbf{A}}^{-1} \cdot \mathbf{M}(0)=\frac{1}{N} \frac{L}{1-\kappa L} .
$$

Here we assume that all sites have same initial magnetization and $\mathrm{N}$ is number of sites.

So far, we have only delt with different isotropic sites or one specific orientation of a molecule with respect to the laboratory frame. In solid, we usually need to consider the chemical exchange process, or molecular reorientation, in a powder sample, which was first studied by Spiess $[148,149,150]$ in NMR although the lineshape analysis methods were first demonstrated in ESR spectroscopy by Sillescu and co-worders $[151,152]$. Each site is frequently subjected to at least one anisotropic interaction (e.g. chemical anisotropy, dipolar coupling between sites, and quadrupolar coupling if the spin of each site is larger than $1 / 2$, etc.). We are particularly interested in the chemical exchange process in molecules with chemical shift anisotropies. In this case, the resonance frequencies which label the sites in molecules are determined by the orientations of the principle axis systems with respect to the molecular frame, the principal values of chemical shift anisotropy, and the orientations of the molecule frames with respect to the laboratory frame. The transformations between the laboratory frame (LAB) and the molecule frame (MOL), and between the MOL frame and the principle axis system (PAS) are illustrated by Fig.(3.3). We now can represent the frequency of $j$-th site as

$$
\omega_{j}=\sum_{m=-2}^{2} \mathcal{D}_{m, 0}^{(2)}\left(\Omega_{M}\right) \omega_{j, m}\left(\Omega_{j}\right)
$$

where

$$
\omega_{j, m}\left(\Omega_{j}\right)=\sum_{m^{\prime}} \mathcal{D}_{m, 0}^{(2)}\left(\Omega_{j}\right) \rho_{2, m^{\prime}}
$$

and the $\Omega_{j}$ are the three Euler angles from the MOL frame to the PAS and the $\Omega_{M}$ from the LAB frame to the MOL frame, $\mathcal{D}_{m, 0}^{(2)}$ are the components of Wigner rotation 
matrix. The $\rho_{2, m}$ are the principal values of the chemical shift anisotropy with values

$$
\rho_{2,0}=\sqrt{\frac{3}{2}} \delta, \quad \rho_{2, \pm 1}=0, \quad \rho_{2, \pm 2}=\frac{1}{2} \eta \delta,
$$

where $\delta$ is the anisotropy and $\eta$ is asymmetry factor. We have assumed that the principal values are same for all sites.

After chemical exchange, the lineshape given by Eq.(5.9) will be similar to liquid situation except for the orientation dependence of the molecules in the laboratory frame. For a powder sample, the overall lineshape is given by

$$
M(\omega)=\frac{1}{4 \pi} \int M\left(\omega, \Omega_{M}\right) \sin \theta d \theta d \phi .
$$

This lineshape is more compiicated than in liquids and also more interesting. As a simple example let us first consider two-site jumps of a water molecule $\left(\mathrm{H}_{2} \mathrm{O}\right)$ in a hydrate sample. From the measurement of the lineshape of ice with a chemical shift anisotropy by multi-pulse technique [153], it was shown that the proton shielding tensor is axially symmetric about the bond direction. The angle between two bonds is $109.5^{\circ}$. The two protons of the water molecule are assumed to perform $180^{\circ}$ jumps about an axis which bisects the bonding angle. Fig.(5.3) shows the lineshapes with different exchange rates. In the very slow motion limit, the lineshape is almost same as the axial-symmetric lineshape while at fast motion limit, it becomes completely asymmetric. Between these two extremes, the lineshapes are distorted. One thing we can see is that as the exchange rate increases two additional singularities appear and gradually dominate the features of the lineshape.

From the standard method [115] of lineshape analysis, the singularities of a powder lineshape are given by

$$
\frac{\partial \omega(\theta, \phi)}{\partial \theta}=0, \frac{\partial \omega(\theta, \phi)}{\partial \phi}=0,
$$

where $\omega(\theta, \phi)$ is the resonance frequency of the magnetization. Applied to our case, the resonance frequencies have to replaced by the eigenvalues of the evolution matrix 

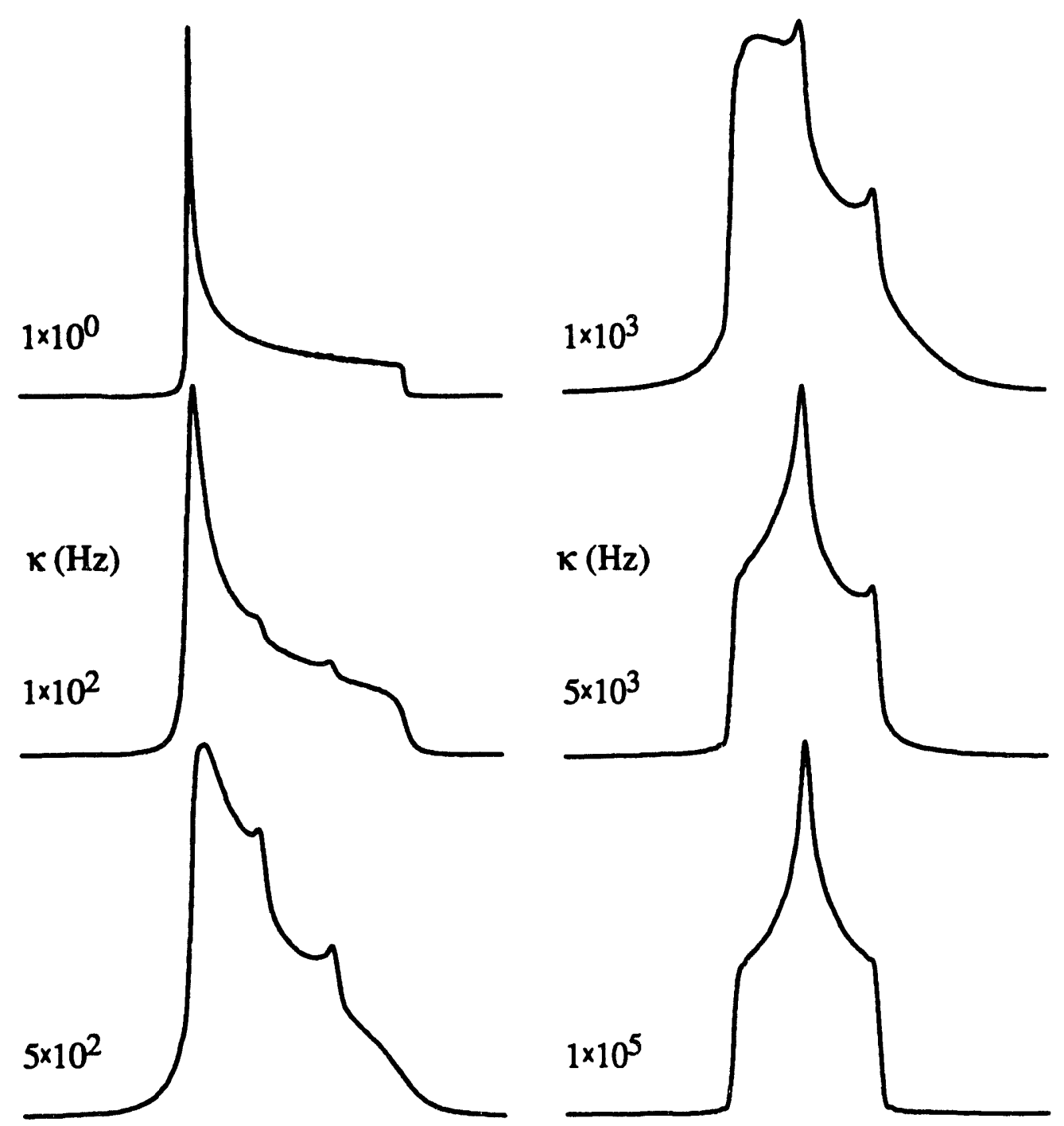

Figure 5.3: Variation of the powder lineshapes with different jumping rate in two-site jumps on a magic angle cone (e.g. two protons jump in $\mathrm{H}_{2} \mathrm{O}$ in solid state). In simulation, the spectrum width is $S W=10 \mathrm{kHz}$, and the principal values of the chemical shift anisotropy are $\delta \omega_{0}=3 k H z$, and $\eta=0$. 
$(\boldsymbol{\imath} \hat{\boldsymbol{\omega}}+\hat{\pi})$. For two-site jumps, the eigenvalues are given by Eq.(5.3) in which the resonance frequencies of the magnetizations of site 1 and 2 in rigid state are

$$
\begin{aligned}
& \omega_{1}(\theta, \phi)=\sum_{m} d_{m, 0}^{(2)}(\theta) e^{-3 m \phi} d_{0, m}^{(2)}\left(\theta^{(2)}\right) \rho_{2,0} \\
& \omega_{2}(\theta, \phi)=\sum_{m}(-1)^{m} d_{m, 0}^{(2)}(\theta) e^{-\imath m \phi} d_{0, m}^{(2)}\left(\theta^{(2)}\right) \rho_{2,0}
\end{aligned}
$$

where the $d_{m, m^{\prime}}^{(2)}$ are the components of reduced Wigner rotation matrix, and $\theta^{(2)}=$ $54.74^{\circ}$, the so-called magic angle. Inserting Eq.(5.24) into Eq.(5.3) yields

$$
\lambda_{ \pm}=\frac{1}{2}\left\{\imath \rho_{2,0} \sin ^{2}(\theta) \cos (2 \phi)-2 \kappa \pm \sqrt{4 \kappa^{2}-2 \rho_{2,0}^{2} \sin ^{2}(2 \theta) \cos ^{2}(\phi)}\right\} .
$$

Substitutes Eq.(5.25) into Eq.(5.23), four singularities can be obtained in agreement with the simulated lineshapes shown in Figure (5.3). These four singularities are given in Table (5.1). In each of the first three singularities, there are two components with same imaginary values (resonance frequencies): one does not disperse and the other disperses according to the rate $2 \kappa$ during the chemical exchange. The last singularity only exists in the region $0 \leq \kappa \leq \frac{1}{\sqrt{2}} \rho_{2,0}$. The two components in this singularity have their own frequencies but with common line broadening factor. The variations of the frequencies of the two components with the exchange rate is same as in Figure (5.1).

Table 5.1: Singularities in a powder lineshape of spin nuclei with chemical anisotropic interaction under two-site jumping

\begin{tabular}{|c|c|c|}
\hline \hline 0 & $\phi$ & $\lambda_{p m}$ \\
\hline 0 & any $\phi$ & $-\kappa \pm \kappa$ \\
\hline$\frac{\pi}{2}$ & 0 & $\frac{1}{2}\left[\imath \rho_{2,0}-2 \kappa \pm 2 \kappa\right]$ \\
\hline$\frac{\pi}{2}$ & $\frac{\pi}{2}$ & $\frac{1}{2}\left[-\imath \rho_{2,0}-2 \kappa \pm 2 \kappa\right]$ \\
\hline $\cos ^{2}(2 \theta)=\frac{-4 \kappa^{2}+2 \rho_{2,0}^{2}}{18 \rho_{2,0}^{2}}$ & 0 & $\frac{2}{2}\left(\rho_{2,0} \pm \sqrt{\rho_{2,0}^{2}-2 \kappa^{2}}\right)-\kappa$ \\
\hline
\end{tabular}




\subsection{Symmetry Properties of Discrete Jumps}

So far we have not considered symmetry effects in the chemical exchange process. In many cases, a molecule has a symmetric shape. This symmetry will dominate all the physical process in the molecule if there are no external perturbations to destroy it. Discrete jumps from site to site are therefore restricted by the molecular symmetry. In this section, we briefly summarize the effects of molecular symmetry on the chemical exchange process, using symmetry group theory proposed by Alexander and his coworkers [143].

We first assume that the local symmetry group of an undestorted molecule is G. All jumping sites $\Omega_{i}(i=1,2, \cdots, N)$ are then related by the elements of the symmetry group. The magnetization at site $j$ for fixed orientation $\Omega_{M}$ forms the basis of the representation (reducible) of the group $G$, that is

$$
M\left(\omega_{j}, \Omega_{M}\right)=M\left(\mathbf{R}^{-1} \omega_{i}, \Omega_{M}\right)=\mathbf{R} M\left(\omega_{i}, \Omega_{M}\right),
$$

where $\mathbf{R}$ is an element of the group $G$. Thus each element $\kappa_{i, j}$ of the exchange rate matrix $\hat{\pi}$ can be associated with a definite element of the group, $\kappa_{i, j}=\kappa(\mathbf{R})$ because $\Omega_{j}=\mathbf{R} \Omega_{i}$. Moreover since $\kappa(\mathbf{R})$ is uniquely determined by the group operation $\mathbf{R}$, the jumping rates corresponding to all equivalent elements, that is those belonging to the same class $C$ in the symmetry group $G$, are same. Furthermore, from detailed balance, Eq.(5.7), we also have $\kappa(\mathbf{R})=\kappa\left(\mathbf{R}^{-1}\right)$ so that the conjugate classes always have the same jumping rate $\kappa$. Occasionally $\mathbf{R}$ and $\mathbf{R}^{-1}$ will not belong to the same class. In this case, there should be a higher symmetry group than $G$, and we can use the larger symmetry group to handle the exchange rate matrix. We assume below that this transformation has already been done.

The exchange rate matrix $\hat{\pi}$ can also be used as an operator, and its $i$-th component, after it has been applied to the $i$-th magnetization, can be written

$$
\kappa_{i} M\left(\Omega_{i}, \Omega_{M}\right)=\sum_{j} \kappa_{i, j} M\left(\Omega_{j}, \Omega_{M}\right)=\sum_{c} \kappa_{c} \sum_{\alpha \in c}\left(\mathbf{R}_{\alpha}-1\right) M\left(\Omega_{i}, \Omega_{M}\right)
$$


where $\kappa_{i}=\left(\kappa_{i, 1}, \kappa_{i, 2}, \cdots, \kappa_{i, N}\right)$, and $\kappa_{c}$ is the jumping rate for the class $C$ of the symmetry group $G$. Now we assume that the basis functions for the irreducible representation of the symmetry group $G$ are $\Psi_{\mu}^{\lambda}\left(\Omega_{M}\right)$ where $\lambda$ means the $\lambda$-th irreducible representation, and $\mu$ is an index of the row in a multi-dimensional representation. These basis functions still depend on the orientations of the molecule frames relative to the LAB frame because all orientations of the molecules have equal probability in a powder sample. This symmetry is determined by the group, $S O(3)$, and is not relevant to the current problem. The magnetization at site $i$ then can be expanded in terms of the basis functions of $G$.

$$
M\left(\Omega_{i}, \Omega_{M}\right)=\sum_{\lambda \mu} a_{i, \mu}^{\lambda} \Psi_{\mu}^{\lambda}\left(\Omega_{M}\right)
$$

The coefficients $a_{i, \mu}^{\lambda}$ are uniquely determined by the symmetry group $G$, e.g. in terms of the projection operators onto the group elements. From the theory of representations of a symmetry group, we have

$$
\sum_{\alpha \in c} \mathbf{R}_{\alpha} \Psi_{\mu}^{\lambda}\left(\Omega_{M}\right)=n_{c} \frac{\chi_{c}^{\lambda}}{\nu_{\lambda}} \Psi_{\mu}^{\lambda}\left(\Omega_{M}\right)
$$

where $n_{c}$ is the order of the class $C, \chi_{c}^{\lambda}$ is the character, and $\nu_{\lambda}$ is the dimensionality of the $\lambda$ irreducible representation of the group G. Applying Eq.(5.29) to Eq.(5.27) yields

$$
\kappa_{i} M\left(\Omega_{i}, \Omega_{M}\right)=-\sum_{\lambda} \kappa_{\lambda} \sum_{\mu} a_{i, \mu}^{\lambda} \Psi_{\mu}^{\lambda}
$$

where

$$
\kappa_{\lambda}=\sum_{c} n_{c} \kappa_{c}\left(1-\frac{\chi_{c}^{\lambda}}{\nu_{\lambda}}\right) .
$$

Now we wish to apply these results of group theory to find the steady state solution of Eq.(5.17). The first step is to transform Eq.(5.7) from the time domain into the frequency domain by use of the Fourier transformation. Each component of the magnetization vector $M$ satisfies

$$
\left[\imath\left(\omega-\omega_{i}\right)-\kappa_{i}\right] M_{i}\left(\Omega_{i}, \Omega_{M}\right)=\imath M_{i}(0) .
$$


From Eqs. (5.30) and (5.28), we have

$$
\sum_{\lambda}\left[2\left(\omega-\omega_{i}\right)-\kappa_{\lambda}\right] M_{i, \lambda}=\imath M_{i}(0)
$$

where

$$
M_{i, \lambda}=\sum_{\mu} a_{i, \mu}^{\lambda} \Psi_{\mu}^{\lambda} .
$$

The lineshape observed experimentally is actually determined by the totally symmetric representation $\left(A_{1}\right)$ of the group $G$ because only the $A_{1}$ representation is orientation independent. Therefore we only need to find the projection of the total magnetization onto the totally symmetric representation, that is $M_{A_{1}}$. To solve Eq.(5.33) for $M_{A_{1}}$ is still very complicated because the left side of Eq.(5.33) is not, in general, block diagonal in the basis of the irreducible representations of the group $G$. This point will be seen more clearly later. However, in many cases, the frequency $\omega_{i}$ of the $i$-th site has its own local symmetry properties. For example, the CSA tensor of the chemical interaction always has a $D_{2}$ symmetry. Once the local symmetry group $S$ for each site is a subgroup of the molecular symmetry group $G$, the calculation of $M_{A_{1}}$ from Eq.(5.33) can possibly be simplified. This simplification occurs is because we need to consider only those irreducible representations which contain the totally symmetric representation of the subgroup $S$. These representations are called the relevant representations, and all others are irrelevant. The restriction to the relevant representations often considerably simplifies the whole calculation. If $S$ contains just the identity of $G$ then no simplification can be done. On the other hand, if $S$ is same as $G$, then the spectrum is totally invariant to the motion, and no parameters are needed because $\kappa_{A_{1}}=0$ always.

As a simple example to illustrate the above statements, let us consider that only one relevant representation in $M_{i}$ beyond the identity representation $\left(A_{1}\right)$, that is

$$
M_{i}\left(\Omega_{M}\right)=M_{A_{1}}+M_{i, \lambda}
$$


After we find the relevant representation $M_{i, \lambda}$, and insert it into Eq.(5.33), we have

$$
\imath\left(\omega-\omega_{i}\right) M_{i}\left(\Omega_{M}\right)-\kappa_{\lambda}\left(M_{i}\left(\Omega_{M}\right)-M_{A_{1}}\left(\Omega_{M}\right)\right)=\imath M_{i}(0)
$$

or

$$
M_{i}\left(\Omega_{M}\right)=\frac{\imath M_{i}(0)-\kappa_{\lambda} M_{A_{1}}\left(\Omega_{M}\right)}{\imath\left(\omega-\omega_{i}\right)-\kappa_{\lambda}} .
$$

The the total magnetization is

$$
M\left(\omega, \Omega_{M}\right)=\sum_{i} M_{i}\left(\omega, \Omega_{M}\right)=N M_{A_{1}}\left(\Omega_{M}\right)=\sum_{i} \frac{2 M_{i}(0)-\kappa_{\lambda} M_{A_{1}}\left(\Omega_{M}\right)}{2\left(\omega-\omega_{i}\right)-\kappa_{\lambda}}
$$

and we may finally obtain a solution identical to Eq.(5.18) which was obtained by using matrix manipulations in the strong collision limit. As can be seen, this procedure is much simpler than the matrix manipulation. Moreover, in many cases, even if there are more than one relevant representation, a group theoretical treatment can still simplify the calculation of the chemical exchange process.

In order to demonstrate the above statements more clearly, we now derive an explicit general equation for $M_{A_{1}}$ that eventually will results in the lineshape of a powder sample. We follow the expansion method given by Freed, Bruno and Polnaszek (FBP)[154], but use our own notation. According to FBP theory, we can explicitly represent the orientation dependence of the $i$-th magnetization $M_{i}\left(\Omega_{i}, \Omega_{M}\right)$ by using a complete set of Wigner rotation matrix elements.

$$
M_{i}\left(\Omega_{i}, \Omega_{M}\right)=\sum_{l, m, m^{\prime}} \frac{(2 l+1)^{\frac{1}{2}}}{8 \pi^{2}} A_{m, m^{\prime}}^{l, i} \mathcal{D}_{m, m^{\prime}}^{(l)}\left(\Omega_{M}\right)
$$

where $A_{m, m^{\prime}}^{l, i}$ is function of $\omega_{i}$ and its value is given by the inverse transformation.

$$
A_{m, m^{\prime}}^{l, i}=\frac{(2 l+1)^{\frac{1}{2}}}{8 \pi^{2}} \int d \Omega_{M} \mathcal{D}_{m, m^{\prime}}^{(l)} \dagger^{\dagger}\left(\Omega_{M}\right) M_{i}\left(\Omega_{i}, \Omega_{M}\right)
$$

The exchange rate matrix $\hat{\pi}$ then couples the $A_{m, m^{\prime}}^{l, i}$. This procedure does not take into account the symmetry of the problem. For $N$ different sites, there will be a total of $N(2 l+1)$ equations for each $l$. In order to take advantage of the symmetry properties of the problem, we notice that $\Omega_{i}$ and $\Omega_{j}$ are related by the jump $\mathbf{R}_{i j}$ from 
site $i$ to $j$. Such a process can also be implemented by rotating the molecular frame from $\Omega_{M}$ to $R_{i j}^{-1} \Omega_{M}$. In other words, Euler angles $\Omega_{i}$ themselves are determined by the orientation of the molecular frame with respect to the laboratory frame, that is $\Omega_{i}=\Omega_{i}\left(\Omega_{M}\right)$. Now we can expand the $i$ th magnetization $M_{i}\left(\Omega_{i}, \Omega_{M}\right)$ in terms of $\Omega_{i}\left(\Omega_{M}\right)$.

$$
M_{i}\left(\Omega_{i}, \Omega_{M}\right)=\sum_{l, m, m^{\prime}} \frac{(2 l+1)^{\frac{1}{2}}}{8 \pi^{2}} A_{m, m^{\prime}}^{l} \mathcal{D}_{m, m^{\prime}}^{(l)}\left(\Omega_{i}\right)
$$

Obviously

$$
\mathcal{D}_{m, m^{\prime}}^{(l)}\left(\Omega_{i}\right)=\sum_{m^{\prime \prime}} \mathcal{D}_{m, m^{\prime \prime}}^{(l)}\left(\Omega_{j}\right) \mathcal{D}_{m^{\prime \prime}, m^{\prime}}^{(l)}\left(\Omega_{M}\right)
$$

Substituting it in Eq.(5.26) gives

$$
\kappa_{i} M_{i}\left(\Omega_{i}, \Omega_{M}\right)=\sum_{c} \kappa_{c} \sum_{l, m, m^{\prime}} \frac{(2 l+1)^{\frac{1}{2}}}{8 \pi^{2}} A_{m, m^{\prime}}^{l} \sum_{\alpha \in c}\left(\mathbf{R}_{\alpha}-1\right) \mathcal{D}_{m, m^{\prime}}^{(l)}\left(\Omega_{i}\right) .
$$

We now expand the $\mathcal{D}_{m, m^{\prime}}^{(l)}\left(\Omega_{i}\right)$ in the basis function $\Psi_{\mu, m}^{\lambda, l}\left(\Omega_{i}\right)$ of the group $G$.

$$
\mathcal{D}_{m, m^{\prime}}^{(l)}\left(\Omega_{i}\right)=\sum_{\lambda, \mu} a_{\mu, m}^{\lambda, l} \Psi_{\mu, m^{\prime}}^{\lambda, l}\left(\Omega_{i}\right) .
$$

Inserting Eq.(5.44) into Eq.(5.43) and using of Eq.(5.29) gives

$$
\kappa_{i} M_{i}\left(\Omega_{i}, \Omega_{M}\right)=\sum_{c} n_{c} \kappa_{c} \sum_{l, m, m^{\prime}} \frac{(2 l+1)^{\frac{1}{2}}}{8 \pi^{2}} A_{m, m^{\prime}}^{l}\left[\sum_{\lambda, \mu} a_{\mu, m}^{\lambda, l} \frac{\chi_{c}^{\lambda}}{\nu_{\lambda}} \Psi_{\mu, m^{\prime}}^{\lambda, l}\left(\Omega_{i}\right)-\mathcal{D}_{m, m^{\prime}}^{(l)}\left(\Omega_{i}\right)\right] .
$$

The coefficients, $a_{\mu, m}^{\lambda, l}$ are the coupling coefficients between two sets of representations (like those between product space and coupled space in the quantum mechanics of angular momentum), and they satisfy orthogonality condition. From Eq.(5.44), we have

$$
\Psi_{\mu, m^{\prime}}^{\lambda, l}\left(\Omega_{i}\right)=\sum_{m} a_{\mu, m}^{\lambda, l} \dagger \mathcal{D}_{m, m^{\prime}}^{(l)}\left(\Omega_{i}\right)
$$

Furthermore, the frequency of the $i$-th site can also be expanded in terms of Wigner rotation matrices.

$$
\omega_{i}\left(\Omega_{i}\right)=\sum_{m} \rho_{2, m} \mathcal{D}_{m, 0}^{(2)}\left(\Omega_{i}\right)
$$


and

$$
\omega_{i}\left(\Omega_{i}\right) M_{i}\left(\Omega_{i}, \Omega_{M}\right)=\sum_{l, m, m^{\prime}, m^{\prime \prime}} \rho_{2, m^{\prime \prime}} A_{m, m^{\prime}}^{l} \mathcal{D}_{m^{\prime \prime}, 0}^{(2)}\left(\Omega_{i}\right) \mathcal{D}_{m, m^{\prime}}^{(l)}\left(\Omega_{i}\right) .
$$

From the product properties of two Wigner rotation matrices, we have

$$
\mathcal{D}_{m_{1}, n_{1}}^{l_{1}} \mathcal{D}_{m_{2}, n_{2}}^{l_{2}}=\sum_{l=\left|l_{1}-l_{2}\right|}^{l_{1}+l_{2}} C\left(l_{1}, l_{2}, l, m_{1}, m_{2}\right) C\left(l_{1}, l_{2}, l, n_{1}, n_{2}\right) \mathcal{D}_{m_{1}+m_{2}, n_{1}+n_{2}}^{l},
$$

where $C\left(l_{1}, l_{2}, l, m_{1}, m_{2}\right)$ is Clebsch-Gordan coefficients. By use of equations from (5.45) to (5.49), Eq.(5.32) becomes

$$
\left(\imath \omega+\frac{1}{T_{2}}\right) A_{m, m^{\prime}}^{l}+\sum_{k} \kappa_{k, m, m^{\prime}}^{l} A_{k, m^{\prime}}^{l}+\sum_{l^{\prime}, m^{\prime \prime}} B_{m-m^{\prime \prime}, m^{\prime}}^{l, l^{\prime}} A_{m-m^{\prime \prime}, m^{\prime}}^{l^{\prime}}=M_{i}(0) \delta(l),
$$

where

$$
\kappa_{k, m, m^{\prime}}^{l}=\sum_{c} n_{c} \kappa_{c}\left[\sum_{\lambda, \mu} \frac{\chi_{c}^{\lambda}}{\nu_{\lambda}} a_{\mu, m}^{\lambda, l} a_{\mu, m}^{\lambda, l} \dagger-\delta_{k, m}\right],
$$

and

$$
B_{m-m^{\prime \prime}, m^{\prime}}^{l, l^{\prime}}=\rho_{2, m^{\prime \prime}} C\left(2, l^{\prime}, l, m^{\prime \prime}, m-m^{\prime \prime}\right) C\left(2, l^{\prime}, l, m^{\prime}, 0\right) .
$$

In the left side of Eq.(5.50), the first and second terms are obviously diagonal, and the second term, which contains the chemical exchange rates, mixes with different elements in the same irreducible representation. The third terms are no longer diagonal and mix different irreducible representations because of the coupling between magnetization and its resonance frequency. If there is no local symmetry group for each site, Eq.(5.50) cannot be reduced further to diagonal or block diagonal form because the basis functions are already irreducible. On the other hand, if the local symmetry group is a subgroup of the group $G, \mathrm{Eq} .(5.50)$ can be rewritten in the basis functions of the subgroup $S$. We assume that this has already been done before the derivation of the Eq.(5.50), and therefore, $A_{m, m^{\prime}}^{l}$ can be found from this equation. In other words, in the Eq.(5.50), the summation over $\lambda$ is only for the relevant representations. Furthermore, one more relevant representation can be removed after rearranging the jump term. As it can be seen from Eq.(5.50), there is no index to specify a particular site. This tells us that the information about the jumps is completely contained in the coefficients $a_{\mu, m}^{\lambda, l}$. In practice, these coefficients therefore have 
to be explicitly computed. Moreover, the lineshapes observed in the NMR spectra are proportional to $A_{0,0}^{0}$. This quantity can be calculated from Eq.(5.50) by truncating the series at some suitable value of $l[154]$. On the one hand, the accuracy of the calculation of $A_{0,0}^{0}$ will increases as $l$ increases while the number of mixing terms $A_{k, m^{\prime}}^{l}$ is increases roughly by $l^{2}$. However, the size of the diagonal jump terms does not increase with $l$ so they do not effect convergence. To obtain good convergence it is necessary to have some non-zero rate (e.g. $T_{2}$ ) for the molecular tumbling.

In practical applications of the above procedure for calculating the effects of the chemical exchange, we have to determine the group $G$ and its subgroup $S$. It is relatively easy to assign group $G$ if we know the symmetry of a molecule. For the chemical shift anisotropy (CSA) tensor with a nonzero asymmetry parameter $\eta$ the local symmetry is the $D_{2}$ group as can be seen from the fact that the CSA tensor does not change after applications of $\pi$ rotations around three orthogonal axes ( $x$, $y, z)$ in the PAS. If the asymmetry parameter is zero, the local symmetry therefore is determined by product group of $D_{2}$ and $C_{\infty}(z)$, that is, $D_{2} \otimes C_{\infty}(z)$ at least. The group $S$ then should be a subgroup of both the local symmetry group and the molecule symmetry group $G$. However, sometimes, the group $S$ is determined by the symmetry of the orientations along which the molecule is distorted by the discrete jumps[143]. Once we know the molecular symmetry group $G$, and the subgroup $S$ under which the Hamiltonian is an invariant, the next step is to find out the relevant representations. This can be obtained from the orthogonality theorem of group theory. The number of an irreducible representation of the subgroup $S$, contained in an irreducible representation of the group $G$ is given by

$$
a_{\lambda, \Gamma}=\sum_{\mathbf{R} \in S} \chi_{\lambda}^{G}(\mathbf{R}) \chi_{\Gamma}^{S}(\mathbf{R})
$$

If $a_{\lambda, A_{1}}$ is larger than zero, representation $\lambda$ of $G$ is relevant, otherwise it is irrelevant. Once we know the number, $N_{r}$, of the relevant representations of $G$, the number of rate parameters in the chemical exchange process is also determined, that is $N_{r}-1$ 
since $\kappa_{A_{1}}=0$ always.

The third step is to determine the coupling coefficients $a_{\mu, m}^{\lambda, l}$. Physically, the coupling coefficients represent the projections of the elements $\mathcal{D}_{m, m^{\prime}}^{(l)}$ of the Wigner rotation matrices, which are the irreducible representations of $S O(3)$ group, along the basis functions, $\Psi_{\mu, m^{\prime}}^{\lambda, l}$, of the irreducible representations of the group $G$. In principle, once we known the basis functions of all irreducible representations of $G$ it is straightforward to calculate the coupling coefficients from the inverse transformation of Eq.(5.46). Multiplying both sides of Eq.(5.46) by $\mathcal{D}_{\mu^{\prime}, m^{\prime}}^{\lambda^{\prime}, l^{\prime}}\left(\Omega_{i}\right)$, and then summing over all possible orientations and all $m^{\prime}$ yields

$$
a_{\mu, m}^{\lambda, l}=\sum_{m^{\prime}, i} \mathcal{D}_{m, m^{\prime}}^{l}\left(\Omega_{i}\right) \Psi_{\mu, m^{\prime}}^{\lambda, l}\left(\Omega_{i}\right)
$$

where the orthogonality condition for the basis functions of the group has been used.

$$
\sum_{i \in G, m^{\prime}} \Psi_{\mu, m^{\prime}}^{\lambda, l}\left(\Omega_{i}\right) \Psi_{\mu^{\prime}, m^{\prime}}^{\lambda^{\prime}, l^{\prime}}\left(\Omega_{i}\right)=\delta_{\lambda, \lambda^{\prime}} \delta_{\mu, \mu^{\prime}} \delta_{l, l^{\prime}}
$$

For the $A_{1}$ representation, it is extremely simple to calculate $a_{0, m}^{A_{1, l} l}$ because that the basis functions are equal ones. Thus $\Psi_{0, m^{\prime}}^{A_{1} l}\left(\Omega_{i}\right)$ is proportional to spherical harmonics, that is

$$
a_{0, m}^{A_{1}, l}=B_{N} \sum_{i}(-1)^{m} Y_{m}^{l}\left(\theta_{i}, \phi_{i}\right)
$$

where $B_{N}$ is a normalization constant, and summation is over all possible operations of the group $G$.

However for other representations of $G$, it is difficult to have a complete set of basis functions. Fortunately, for most simple symmetry groups, all the representations are listed in text books on group theory $[68,156]$. A general procedure, introduced by Golding [161], for determining these coefficients with double valued groups is based on the Clebsch-Gordan series for the product of two irreducible representations of a group and the correspondence between the angular momentum and the irreducible representations. We briefly summarize this procedure here. First we realize that the coupling coefficients $a_{\mu, m}^{\lambda, l}$ form the transformation from the $l$ representation $\mathcal{D}_{m^{\prime}, m}^{(l)}$ of 
$S O(3)$ to the $\lambda$ representation of the group $G$. It will be seen that the coupling coefficients are related to the Clebsch-Gordan coefficients or the $V$ coefficients between the coupling of two irreducible representation of the group $G$. In order to compare our results with those of Golding, we use a ket $|\lambda \mu\rangle$ to represent the basis function $\Psi_{\mu}^{\lambda}$ of the irreducible representation $\lambda$ of the group $G$. In order to be general, $|\lambda \mu\rangle$ is usually defined by a complex function.

Now we define the relationship between $\mid \lambda \mu>$ and $\mid l m>$ for a specific $l$ value as

$$
\left|l \lambda \mu>=\sum_{m} a_{\mu, m}^{\lambda, l}\right| l m>.
$$

Since we have defined $|\lambda \mu\rangle$ to be complex, the coupling coefficients can be chosen to be real numbers which depend only on $l, m, \lambda$, and $\mu$, and which satisfy the orthogonality conditions.

$$
\sum_{m} a_{\mu, m}^{\lambda, l} a_{\mu^{\prime}, m}^{\lambda^{\prime}, l}=\delta_{\lambda, \lambda^{\prime}} \delta_{m u, m u^{\prime}}
$$

and

$$
\sum_{\lambda, \mu} a_{\mu, m}^{\lambda, l} a_{\mu, m^{\prime}}^{\lambda, l}=\delta_{m, m^{\prime}}
$$

The inverse form of Eq.(5.57) is

$$
\left|l m>=\sum_{\lambda, \mu} a_{\mu, m}^{\lambda, l}\right| l \lambda \mu>
$$

As it is well known that the coupling between two angular momentum $\mathbf{L}_{1}$ and $\mathbf{L}_{2}$ to form the third angular momentum $\mathbf{L}_{3}$ is defined by $\mathbf{L}_{1}+\mathbf{L}_{2}=\mathbf{L}$. Correspondingly the relationship among kets $\left|i_{1} m_{1}\right\rangle,\left|l_{2} m_{2}\right\rangle$, and $\mid l_{1} l_{2} l m>$ is given by

$$
\left|l_{1}, m_{1}>\right| l_{2}, m_{2}>=\sum_{l} C\left(l_{1}, l_{2}, l, m_{1}, m_{2}\right) \mid l_{1} l_{2} l m>
$$

and

$$
\left|l_{1} l_{2} l m>=\sum_{m_{1}, m_{2}} C\left(l_{1}, l_{2}, l, m_{1}, m_{2}\right)\right| l_{1}, m_{1}>\mid l_{2}, m_{2}>
$$


where $C\left(l_{1}, l_{2}, l, m_{1}, m_{2}\right)$ are Clebsch-Gordan coefficients. It will then follow by substituting the appropriate expressions of the form of Eqs.(5.57) and (5.60) into (5.62) that

$$
\left|l \lambda \mu>=\sum_{m, m_{1}, m_{2}, \lambda_{1}, \lambda_{2}, \mu_{1}, \mu_{2}} a_{\mu, m}^{\lambda_{1} l} a_{\mu_{1}, m_{1}}^{\lambda_{1}, l_{2}} a_{\mu_{2}, m_{2}}^{\lambda_{2}, l_{2}} C\left(l_{1}, l_{2}, l, m_{1}, m_{2}\right)\right| l_{1} \lambda_{1} \mu_{1}>\mid l_{2} \lambda_{2} \mu_{2}>.
$$

However, the $l$ related function $\mid l \lambda \mu>$ itself is a linear combination of $\left|\lambda_{1} \lambda_{2} \lambda \mu\right\rangle$, that is

$$
\left|l \lambda \mu>=\sum_{\lambda_{1}, \lambda_{2}} C_{l_{1}, l_{2}, l}^{\lambda_{1}, \lambda_{2}}\right| \lambda_{1} \lambda_{2} \lambda \mu>
$$

where coefficients $C_{l_{1}, l_{2}, l}^{\lambda_{1}, \lambda_{2}}$ depend only on $l_{1}, l_{2}, l, \lambda_{1}$, and $\lambda_{2}$. The order of $\lambda_{1}$, $\lambda_{2}$ is important in the $C_{l_{1}, l_{2}, l}^{\lambda_{1}, \lambda_{2}}$ coefficients. Furthermore, the coupling between two irreducible representation is given by

$$
\left|\lambda \mu>=\sum_{\mu_{1}, \mu_{2}} C\left(\lambda_{1}, \lambda_{2}, \lambda, \mu_{1}, \mu_{2}, \mu\right)\right| \lambda_{1} \mu_{1}>\left|\lambda_{2} \mu_{2}\right\rangle
$$

and

$$
\left.\left|\lambda_{1} \mu_{1}>\right| \lambda_{2} \mu_{2}\right\rangle=\sum_{\lambda} C\left(\lambda_{1}, \lambda_{2}, \lambda, \mu_{1}, \mu_{2}, \mu\right) \mid \lambda \mu>,
$$

where the coefficients $C\left(\lambda_{1}, \lambda_{2}, \lambda, \mu_{1}, \mu_{2}, \mu\right)$ are still formally Clebsch-Gordan coefficients, coupling two irreducible representations of the group $G$. The values of these coefficients depend on the particular group and are tabulated in reference[160, 156] for most symmetry groups. These coefficients have all the properties of the common Clebsch-Gordan coefficients. Inserting Eq.(5.65) into (5.64) yields

$$
\left|l \lambda \mu>=\sum_{\lambda_{1}, \lambda_{2}, \mu_{1}, \mu_{2}} C_{l_{1}, l_{2}, l}^{\lambda_{1}, \lambda_{2}} C\left(\lambda_{1}, \lambda_{2}, \lambda, \mu_{1}, \mu_{2}, \mu\right)\right| \lambda_{1} \mu_{1}>\left|\lambda_{2} m u_{2}\right\rangle .
$$

Comparing the coefficients in Eqs.(5.63) and (5.67) yields

$$
C_{l_{1}, l_{2}, l}^{\lambda_{1}, \lambda_{2}} C\left(\lambda_{1}, \lambda_{2}, \lambda, \mu_{1}, \mu_{2}, \mu\right)=\sum_{m, m_{1}, m_{2},} a_{\mu_{1}, l}^{\lambda_{1} l} a_{\mu_{1}, m_{1}}^{\lambda_{1}, l_{1}} a_{\mu_{2}, m_{2}}^{\lambda_{2}, l_{2}} C\left(l_{1}, l_{2}, l, m_{1}, m_{2}\right),
$$

with the condition

$$
\sum_{\mu_{1}, \mu_{2}}\left|C\left(\lambda_{1}, \lambda_{2}, \lambda, \mu_{1}, \mu_{2}, \mu\right)\right|^{2}=1
$$


Hence, the coefficients $a_{\mu, m}^{\lambda_{1} l}, C\left(\lambda_{1}, \lambda_{2}, \lambda, \mu_{1}, \mu_{2}, \mu\right)$, and $C_{l_{1}, l_{2}, l}^{\lambda_{1}, \lambda_{2}}$ are all related by Eq.(5.68). If we know all the coupling coefficients $C\left(\lambda_{1}, \lambda_{2}, \lambda, \mu_{1}, \mu_{2}, \mu\right)$ for a group $G$, and all the $a_{\mu, m}^{\lambda, l}$ coefficients for specific $l_{1}$ and $l_{2}$, the $C_{l_{1}, l_{2}, l}^{\lambda_{1}, \lambda_{2}}$ and $a_{\mu, m}^{\lambda, l}$ coefficients for a specific $l$ value can be determined by using the triangle condition, $\mathbf{L}_{1}+\mathbf{L}_{2}=\mathbf{L}$ of the Clebsch-Gordan coefficients. If, on the other hand, all the coefficients $a_{\mu, m}^{\lambda, l}$ for $l_{1}, l_{2}$, and $l$ are known, we may determine all the $C_{l_{1}, l_{2}, l}^{\lambda_{1}, \lambda_{2}}$ and $C\left(\lambda_{1}, \lambda_{2}, \lambda, \mu_{1}, \mu_{2}, \mu\right)$ coefficients. The coupling coefficients $C\left(\lambda_{1}, \lambda_{2}, \lambda, \mu_{1}, \mu_{2}, \mu\right)$ will, in general, depend on how the irreducible representations of the group $G$ are defined.

One representation of the coupling coefficients between two irreducible representation are the $V$ coefficients $[157,158,159]$ which are similar to Racah's $\bar{V}$. In order to let $V$ coefficients possess properties analogous to Racah's $\bar{V}$ coefficients, its definition with Clebsch-Gordan coefficients $C\left(\lambda_{1}, \lambda_{2}, \lambda, \mu_{1}, \mu_{2}, \mu\right)$ is given by Golding as

$$
V\left(\lambda_{1}, \lambda_{2}, \lambda, \mu_{1}, \mu_{2}, \mu\right)=\frac{(-1)^{2 \lambda_{2}+\lambda+\mu}}{\sqrt{n^{\lambda}}} C\left(\lambda_{1}, \lambda_{2}, \lambda, \mu_{1}, \mu_{2}, \mu\right),
$$

where $n^{\lambda}$ is the dimension of the irreducible representation $\lambda$ of the group $G$. Now we can apply the properties of Racah's coefficients to this $V\left(\lambda_{1}, \lambda_{2}, \lambda, \mu_{1}, \mu_{2}, \mu\right)$ symmetry coupling coefficients, namely:

(a) it is unchanged by an even permutation of the indices,

(b) for an odd permutation, the $V\left(\lambda_{1}, \lambda_{2}, \lambda, \mu_{1}, \mu_{2}, \mu\right)$ coefficients are changed by a factor $(-1)^{\lambda_{1}+\lambda_{2}+\lambda}$,

(c) for a change of sign of subindices $\mu_{1}, \mu_{2}$, and $\mu$, the $V$ coefficients are changed by a factor $(-1)^{\lambda_{1}+\lambda_{2}+\lambda}$.

$$
\begin{aligned}
V\left(\lambda_{1}, \lambda_{2}, \lambda_{3}, \mu_{1}, \mu_{2}, \mu_{3}\right) & =(-1)^{\lambda_{1}+\lambda_{2}+\lambda_{3}} V\left(\lambda_{2}, \lambda_{1}, \lambda, \mu_{2}, \mu_{1}, \mu\right) \\
& =(-1)^{\lambda_{1}+\lambda_{2}+\lambda} V\left(\lambda_{3}, \lambda_{2}, \lambda_{1}, \mu_{3}, \mu_{2}, \mu_{1}\right) \\
& =(-1)^{\lambda_{1}+\lambda_{2}+\lambda_{3}} V\left(\lambda_{1}, \lambda_{2}, \lambda_{3},-\mu_{1},-\mu_{2},-\mu_{3}\right) .
\end{aligned}
$$

From the procedure outlined in above, in principle, all the coupling coefficients, $a_{\mu, m}^{\lambda, l}$ may be calculated one by one with the tables of Clebsch-Gordan coefficients 
between irreducible representations of groups, which are listed in most of text books of group theory $[68,156,160]$. However, since the icosahedral group is complicated and has very few applications, the coupling coefficients have not been calculated. We consider it beyond the range of this work to do so, and leave it as a future project.

\subsection{Computational Approaches and Examples}

In the last two sections, we have described the general theories for calculating chemical exchange processes. In practice, we usually deal with a chemical exchange process with more than two sites and in many cases more than one rate parameter. It is often very difficult to produce an analytical solution for these general chemical exchange problems, and we have to soive them by numerical methods.

We have written a program called CESC (Chemical Exchange with Strong Collision limit) which solve the general chemical exchange problems in the strong collision limit based on the Eqs.(5.18) and (5.22). CESC has versions of FORTRAN 77, VAX PASCAL, and THINK PASCAL for the Macintosh. The orientation of each site is input from the parameter file, and the powder average is done by the Simplex method.

For the problems in the weak collision limit, another program named CEWCIM (Chcinical Exchange with Weak Collision by Inverting Matrix) was written and is based on Eqs.(5.9) and (5.22). Again the powder average is done by the Simplex method, and matrix inversion is performed by the LU decomposition method. The advantage of using matrix inversion method to calculate the lineshapes under the chemical exchanges is that its algorithm is simple, and the accuracy is relatively easy to be controlled by using double precision numbers throughout the numerical calculations. The simulated results are stable. The disadvantage of this method is that one matrix inversion must be done for each value of the frequency. In order to obtain a spectrum, $n p$ ( $n p>100$ usually) points has to be taken. In other 
words, each spectrum is obtained by performing $n p$ matrix inversions. For a powder sample, we need to take $N_{a} \times N_{b}$ orientations $\left(N_{a}\right.$ is number of steps for $\phi$ and $N_{b}$ for $\theta$ ) to have a powder average. Furthermore, each matrix inversion requires $N^{3}$ multiplications. Thus total number of multiplications for a powder averaged spectrum is $n p \times N_{a} \times N_{i} \times N^{3}$. The program therefore is very slow, but with the recent advances in computer technology, spectra can be simulated on work-station computer for up to 60 sites and 10,000 orientations for a powder sample.

In order to bypass the time consuming matrix inversions, Gordon and McGinnis[162] proposed a procedure in which a $\mathrm{QR}$ transformation is first applied to diagonal the non-Hermitian exchange matrix $\imath \hat{\omega}\left(\Omega_{M}\right)+\hat{\pi}$.

$$
\hat{\mathbf{S}}^{-1}\left(\Omega_{M}\right)\left(\imath \hat{\boldsymbol{\omega}}\left(\Omega_{M}\right)+\hat{\boldsymbol{\pi}}\right) \hat{\mathbf{S}}\left(\Omega_{M}\right)=\hat{\boldsymbol{\lambda}}\left(\Omega_{M}\right)
$$

where the $\hat{\mathbf{S}}\left(\Omega_{M}\right)$ is the transformation matrix and $\hat{\lambda}\left(\Omega_{M}\right)$ is the diagonal matrix of the non-Hermitian matrix $\imath \hat{\boldsymbol{\omega}}\left(\Omega_{M}\right)+\hat{\pi}$. Notice that none of the matrices in Eq.(5.72) depend on the frequency. After Fourier transformation of Eq.(5.8), the spectrum is then given by

$$
M\left(\omega, \Omega_{M}\right)=\mathbf{1} \cdot\left(\hat{\mathbf{S}}\left(\Omega_{M}\right)[\omega \hat{\mathbf{1}}-\hat{\lambda}]^{-1}\left(\Omega_{M}\right) \hat{\mathbf{S}}^{-1}\left(\Omega_{M}\right)\right) \cdot \mathbf{M}(0),
$$

which can be evaluated to yield

$$
M\left(\omega, \Omega_{M}\right)=\sum_{i} \frac{\left[\mathbf{1} \cdot \hat{\mathbf{S}}\left(\Omega_{M}\right)\right]_{i}\left[\hat{\mathbf{S}}^{-1}\left(\Omega_{M}\right) \cdot \mathbf{M}(0)\right]_{i}}{\imath \omega-\lambda_{i}\left(\Omega_{M}\right)} .
$$

Thus the whole lineshape of $N$ sites is reduced to a single summation over the $N$ sites once the diagonalization and the inversion of the transformation matrix have been performed. Now the number of the multiplications is reduced to approximately $2 N_{a} \times$ $N_{b} \times N^{3}+3 n p \times N^{2}$ for a powder averaged spectrum. Based on the above algorithm and the QR algorithm in EISPACK, we have written a program called CEWC. The main problem with this program is that the accuracy of the diagonalization of a matrix becomes very bad once the dimension of the matrix is larger than 20 even 
when the calculation is carried out using double precision numbers. I think a better program for matrix diagonalization now exists, such as the QZ algorithm.

An alternative method is the group theoretical treatment of the chemical exchange process. From what we showed previously, Eq.(5.50) can be used to approximately calculate the lineshapes by truncating the series at some suitable $l$ value. As it has be mentioned in the last section, to do that we need to know the coefficients $a_{\mu, m}^{\lambda, l}$. At the end of the last section, we have shown a general procedure for calculating these coefficients, but still we have to know the coupling coefficients $V\left(\lambda_{1}, \lambda_{2}, \lambda, \mu_{1}, \mu_{2}, \mu\right)$ which depend on the properties of the symmetry group $G$ and its irreducible representations. Examples to illustrate application of this procedure can be found in the references $[144,161,145]$ etc.

In many cases, we need to compare the lineshapes owing to rotational diffusion and due to discrete jumps in order to study the motions of a molecule. The lineshape with rotational diffusion may not be calculated exactly with the exchange matrix formalism because its dimension is infinite. However, we can use two methods to yield an approximate lineshape. One is based on the theory proposed by Freed, Bruno, and Polnaszek[154]. The other is that using a very large number of discrete sites to form an exchange matrix whose off-diagonal elements are all equal, that is same as strong collision model. This matrix then can be used to approximately replace the infinite dimension chemical exchange matrix. Thus we can use our program CESC to calculate the lineshapes for rotational diffusion model. Both methods are very good at lineshape simulations. The second method was first used by Wemmer[145].

In order to calibrate the above three programs (CESC, CEWCIM, and CEWC), let us consider chemical exchange process in a molecule with tetrahedron symmetry. Both the experimental and theoretical investigations were first done by Spiess $[148,149,150]$ in solid white phosphorus in the $\beta$-phase at various temperatures. Unfortunately the simulated lineshapes shown in his first paper [149] and 
also in Mehring's book[19] are wrong due to errors in the powder averaging. Later, Wemmer et al studied the dynamical processes in ice. Based on their results, they concluded that the water molecules bond together according to tetrahedral symmetry to form ice.

The CSA tensor in a tetrahedral molecule has a local $D_{2}$ symmetry and the characters of the $D_{2}$ group is tabulated in Table (5.2). Since the CSA tensor of

Table 5.2: Characters of the dihedral group $D_{2}$

\begin{tabular}{|c|rrrr|}
\hline \hline$D_{2}$ & $E$ & $C_{2}(z)$ & $C_{2}(x)$ & $C_{2}(y)$ \\
\hline$A$ & 1 & 1 & 1 & 1 \\
$B_{1}$ & 1 & 1 & -1 & -1 \\
$B_{2}$ & 1 & -1 & 1 & -1 \\
$B_{3}$ & 1 & -1 & -1 & 1 \\
\hline
\end{tabular}

protons in ice has an asymmetry parameter of $\eta=0$, the CSA tensor has even higher symmetry. This new symmetry group is $D_{\infty}$. In the tetrahedron group $T_{d}$, there are two subgroups: $D_{2}, C_{3}$. The characters of $T_{d}$ are listed in Table (5.3). From

Table 5.3: Characters of the tetrahedral group $T, \epsilon=e^{\frac{2 \pi}{3}}$

\begin{tabular}{|c|rrrr|}
\hline$T_{d}$ & $E$ & $4 C_{3}$ & $4 C_{3}^{2}$ & $3 C_{2}$ \\
\hline$A$ & 1 & 1 & 1 & 1 \\
$E$ & 1 & $\epsilon$ & $\epsilon^{*}$ & 1 \\
& 1 & $\epsilon^{*}$ & $\epsilon$ & 1 \\
$T$ & 3 & 0 & 0 & -1 \\
\hline
\end{tabular}

the Tables (5.2) and (5.3), the decomposition of each individual representations of tetrahedral group under its subgroup $D_{2}$ can be performed by use of Eq.(5.53), and is tabulated in Table (5.4). It can be seen that there is only one relevant 
Table 5.4: Decomposition of the representations of the tetrahedral group $T$ under the dihedral group $D_{2}$

\begin{tabular}{|c|c|}
\hline \hline$I$ & $D_{2}$ \\
\hline$A$ & $A_{1}$ \\
$E$ & $2 A_{1}$ \\
$T$ & $B_{1}+B_{2}+B_{3}$ \\
\hline
\end{tabular}

representation beyond the identity representation in the $T_{d}$ group if the subgroup is $D_{2}$. The lineshape during chemical exchange therefore is uniquely determined by a single rate parameter. This rate parameter corresponds to the jumping rates of all $C_{3}$ and $C_{2}$ rotations in the tetrahedral group. Thus, the chemical exchange process satisfies strong collision condition.

Fig.(5.4) shows variations of the lineshapes calculated by CESC, CEWCIM, and CEWC respectively with different rates. At slow exchange rates, the amplitude of the real part in exchange matrix is very small, and the imaginary part dominates the behavior of the diagonalization. In this case, the matrix is in its most unHermitian form, and the accuracy of the diagonalization procedure is the worst. This behavior explains why the lineshape simulated by CEWC is not very smooth at low exchange rates. Otherwise, all the lineshapes simulated with different programs are quite similar, indicating all the programs work well.

In the variation of the lineshapes shown in Figure (5.4) for a tetrahedral molecule, there are two singularities beyond the original two singularities of a powder pattern with a CSA interaction in which the asymmetry parameter is zero. One is always at isotropic frequency with its amplitude, neither the linewidth nor the frequency, depending upon the exchange rate. Both the frequency and the linewidth of the other singularity depend on the exchange rate. In the very slow exchange region, the position of this singularity is at $\frac{\delta}{2}$ and the peak is very sharp. As the exchange 


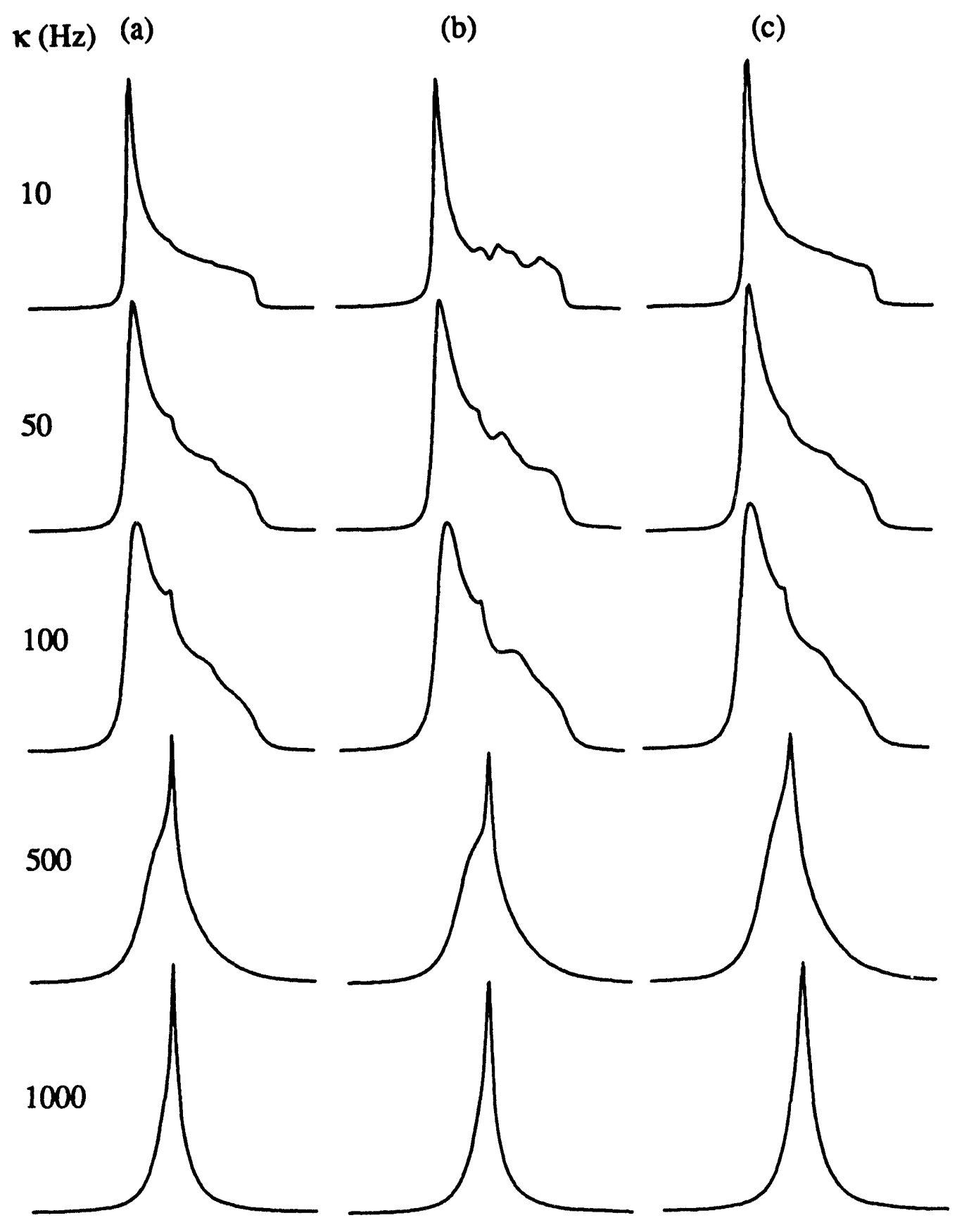

Figure 5.4: Variation of the lineshapes with different exchange rates in a tetrahedron molecule simulated: (a) by CESC, (b) by CEWC, and (c) by CEWCIM programs respectively. The exchange rate is in unit of Hertz; the anisotropy of the CSA tensor is $\omega_{0} \delta=3 \mathrm{kHz}$ and its asymmetry parameter is zero. 
rate increases, the position of the singularity reduces to the isotropic frequency and its linewidth increases. In the fast exchange region, there is only one single sharp peak in the spectrum. All these properties of the lineshape with exchange rate can be explained by use of the singularity analysis method described in chapter 3 and also of section 5.2 .

In the molecular frame, if we choose the three $C_{2}$ rotation axes as the $X_{M}, Y_{M}$, and $Z_{M}$ axes, the frequencies of four sites are

$$
\begin{aligned}
& \omega_{1}(\theta, \phi)=\sum_{m} d_{m, 0}^{(2)}(\theta) d_{0, m}^{(2)}\left(\theta_{m}\right) e^{-\imath m\left(\frac{\pi}{4}+\phi\right)} \rho_{2,0} \\
& \omega_{2}(\theta, \phi)=\sum_{m} d_{m, 0}^{(2)}(\theta) d_{0, m}^{(2)}\left(\theta_{m}\right) e^{-\imath m\left(\frac{5 \pi}{4}+\phi\right)} \rho_{2,0} \\
& \omega_{3}(\theta, \phi)=\sum_{m} d_{m, 0}^{(2)}(\theta) d_{0, m}^{(2)}\left(\pi-\theta_{m}\right) e^{-\imath m\left(\frac{3 \pi}{4}+\phi\right)} \rho_{2,0} \\
& \omega_{4}(\theta, \phi)=\sum_{m} d_{m, 0}^{(2)}(\theta) d_{0, m}^{(2)}\left(\pi-\theta_{m}\right) e^{-\imath m\left(\frac{7 \pi}{4}+\phi\right)} \rho_{2,0}
\end{aligned}
$$

where $\theta_{m}$ is the magic angle, $\theta_{m}=54.74^{\circ}$. Once the $Z_{M}$ axis becomes aligned with the direction of the external magnetic field, $\theta=0^{\circ}$, the frequencies of all four sites are zero. The matrix $\hat{\omega}\left(\Omega_{M}\right)+\hat{\pi}$ is therefore reduced to a real symmetric matrix whose diagonal elements are equal. It will be seen later that one of the four eigenvalues for this $4 \times 4$ matrix is actually zero. Thus this particular orientation corresponds to one of the singularities of the lineshapes during chemical exchange. The frequency of this singularity, which is determined by the imaginary part of the singularity, is zero in the rotating frame, and the line broadening, real part of the singularity, is also zero if we neglect relaxation effects induced by the molecule's random tumbling motions. This explains the sharp peak at the isotropic resonance frequency in the powder lineshapes shown in Figure (5.4). From the above discussion, we can generally conclude that there will be a sharp peak in the spectrum with $N$-site chemical exchange if and only if all sites have a constant frequencies at particular orientation of the molecule with respect to the laboratory frame. The frequency of the sharp peak is sarne as the frequency of all sites. To see this, we assume that all sites have a constant 
frequencies, $\omega_{c}$, and then define $\lambda^{\prime}=w_{c}-\lambda-(N-1) \kappa$. The new eigenvalues $\lambda^{\prime}$ therefore are determined only by the matrix $\hat{\pi}+\left[(N-1) \kappa-\lambda^{\prime}\right] \hat{\mathbf{1}}$ which is obviously real and symmetric, and its diagonal elements are $\lambda^{\prime}$. In order to diagonalize this real symmetric matrix, we use the secular equation.

$$
\begin{aligned}
\operatorname{det}\left[\hat{\omega}\left(\Omega_{M}\right)+\hat{\pi}-\lambda \hat{\mathbf{1}}\right] & =\left|\begin{array}{ccccc}
-\lambda^{\prime} & \kappa & \kappa & \cdots & \kappa \\
\kappa & -\lambda^{\prime} & \kappa & \cdots & \kappa \\
\vdots & \vdots & \vdots & \vdots & \vdots \\
\kappa & \cdots & \cdots & \cdots & -\lambda^{\prime}
\end{array}\right| \\
& =\left(\lambda^{\prime}-\kappa\right)^{N-1}\left[\lambda^{\prime}+(N-1) \kappa\right]=0
\end{aligned}
$$

The solution of Eq.(5.76) is obvious, that is, $\lambda_{1}^{\prime}=-(N-1) \kappa$ or $\lambda=\imath \omega_{c}$, and $\lambda_{i}^{\prime}=\kappa$ or $\lambda_{i}=w_{c}-N \kappa$ for $i=2,3, \cdots, N$. So, we can see that in the first eigenvalue $\lambda_{1}$ there is no real part and we know that the real part corresponds to the line broadening. If there are two different frequencies for $N$ sites, the situation becomes very different. Since the linewidths corresponding to the singularities depend on the exchange rate, the singularities will be smoothed out by this line broadening.

Another example which has the same properties as chemical exchange in a tetrahedral molecule is jumps on a magic angle cone. Fig.(5.5) shows variations of lineshapes with different jumping sites and different jumping rates. A sharp peak at isotropic resonance frequency can still be clearly observed even when the number of the jumping sites on the magic angle cone is thirty. On the magic angle cone, the frequency of $i$ th site is

$$
\omega_{i}(\theta, \phi)=\sum_{m} d_{m, 0}^{(2)}(\theta) d_{0, m}^{(2)}\left(\theta_{m}\right) e^{-\imath m\left(\phi_{i}+\phi\right)} \rho_{2,0} .
$$

When $\theta=0^{\circ}$, all the elements of the reduced Wigner rotation matrices are zero except $m=0$ term. Furthermore $d_{0,0}^{(2)}\left(\theta_{m}\right)=P_{2}\left(\cos \theta_{m}\right)=0$ at magic angle $\theta_{m}=$ $54.74^{\circ}$. Thus at $\theta=0^{\circ}$, the frequencies of all sites on the magic angle cone are zero. Under this condition, we have a singularity to which the chemical exchange does not contribute to the line broadening. This singularity always exists for any number 

(a) 3 sites
(b) 10 sites
(c) 30 sites

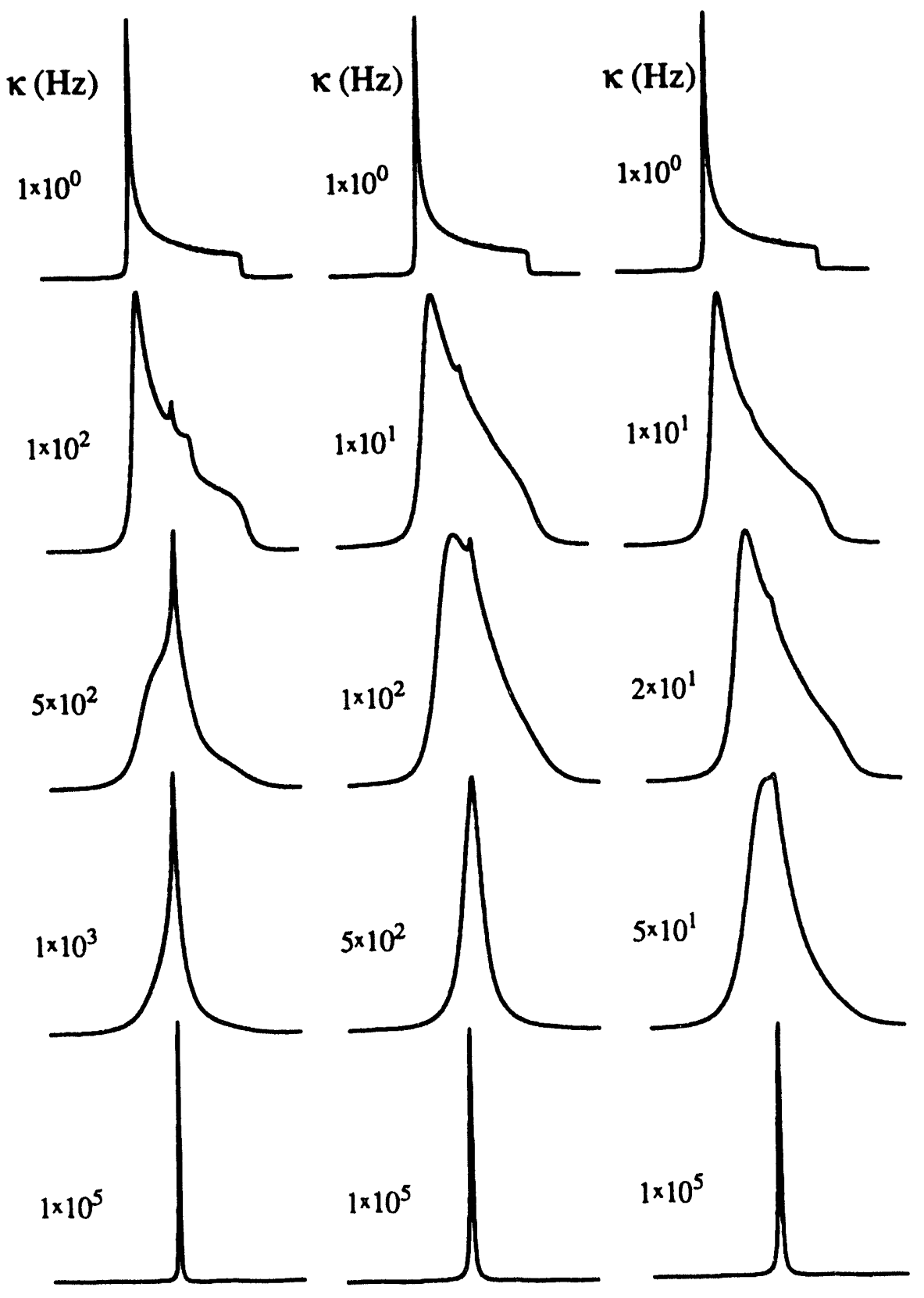

Figure 5.5: Variation of the lineshapes with different exchange rates jumping on a magic angle cone: (a) three sites, (b) ten sites, and (c) thirty sites. The exchange rate is in unit of Hertz; the anisotropy of the CSA tensor is $\omega_{0} \delta=3 k H z$ and its asymmetry parameter is zero. 
of sites on the magic angle cone, only the intensity, determined by the partition in the whole exchange matrix, decreases as increasing the number of sites. This is the reason why we can see a singularity in the spectrum even the number of sites is thirty. In practice, when we use the strong collision model to simulate a lineshape for rotational diffusion case, the number of sites therefore has to be large enough.

\subsection{Jumps with An Icosahedral Symmetry}

As we showed in section 5.3 , the first step in calculating the lineshape with chemical exchange through to use group theory is to determine the number of the relevant representations involved in the molecular symmetry group, $G$. To find the relevant representations of molecular symmetry group $G$, we require the local symmetry group, $S$, of the CSA tensor. This local symmetry group, $S$, is the dihedral group $D_{2}$ in the case of $\eta \neq 0$, and becomes the $D_{\infty}$ once the asymmetry parameter of the CSA tensor is equal zero. If the molecular symmetry group is the icosahedral group, there are five classes: $E$ (identity), $12 C_{5}, 12 C_{5}^{2}, 20 C_{3}$, and $15 C_{2}$ respectively. The rotation axes of the $C_{5}^{2}$ and $C_{5}$ elements are the vertices, those of the $C_{3}$ elements are the centers of the triangles, and those of the $C_{2}$ elements are the centers of the edges of the icosahedron (see Figure.(5.6b)). The icosahedral group contains eight different subgroups: $T, D_{5}, D_{3}, D_{2}, C_{3}, C_{2}$, and $C_{1}$, and it has five irreducible representations: $A_{1}, T_{1}, T_{2}, G$, and $H$. The characters of groups $D_{2}, D_{3}, D_{5}$, and $I$ are tabulated in Tables (5.2), (5.5), (5.6), and (5.7). From these character tables and Eq.(5.53), the decomposition of the representations of the icosahedron group relative to its subgroups $D_{5}, D_{3}, D_{2}$ is given in Table (5.9). In the decomposition of the representation of the icosahedral group under the dihedral group $D_{5}$, there is only one relevant representation in addition to the identity representation while there are two relevant representations under the $D_{3}$ or $D_{2}$. Thus, in the case where the asymmetry parameter, $\eta$, of the CSA tensor is not equal to zero, and the local 


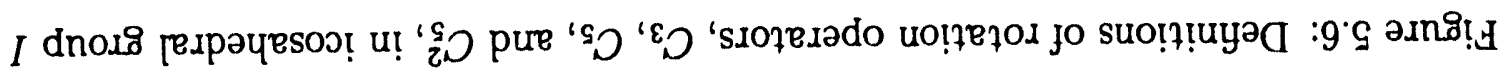

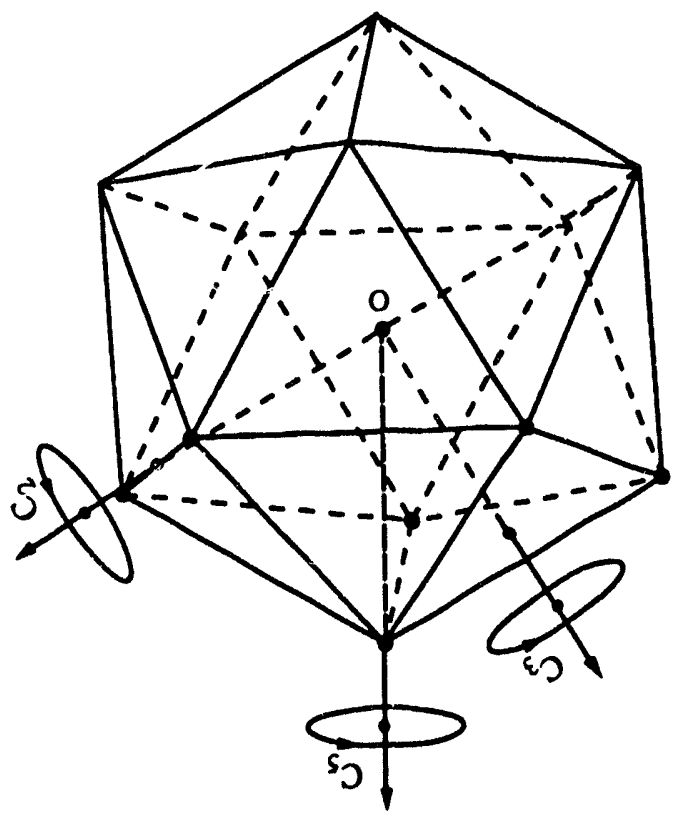

(q)

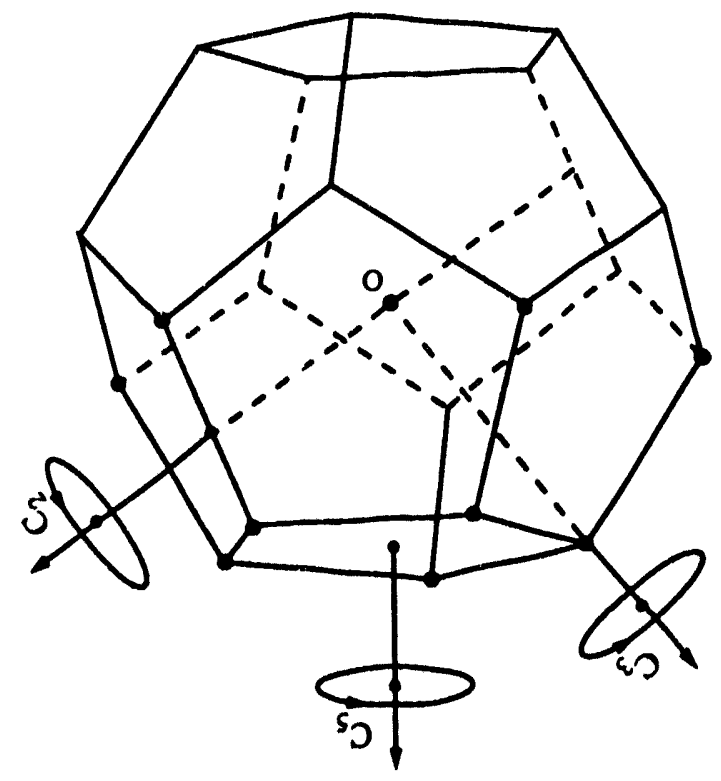

(e 
Table 5.5: Characters of the dihedral group $D_{3}$

\begin{tabular}{|c|ccc|}
\hline \hline$D_{3}$ & $E$ & $2 C_{3}$ & $3 C_{2}$ \\
\hline$A_{1}$ & 1 & 1 & 1 \\
$A_{2}$ & 1 & 1 & -1 \\
$E$ & 2 & -1 & 0 \\
\hline
\end{tabular}

Table 5.6: Characters of the dihedral group $D_{5}$

\begin{tabular}{|c|cccc|}
\hline \hline$D_{5}$ & $E$ & $2 C_{5}$ & $2 C_{5}^{2}$ & $5 C_{2}$ \\
\hline$A_{1}$ & 1 & 1 & 1 & 1 \\
$A_{2}$ & 1 & 1 & 1 & -1 \\
$E_{1}$ & 2 & $2 \cos 72^{\circ}$ & $2 \cos 144^{\circ}$ & 0 \\
$E_{2}$ & 2 & $2 \cos 144^{\circ}$ & $2 \cos 72^{\circ}$ & 0 \\
\hline
\end{tabular}

symmetry is determined only by the dihedral group $D_{2}$, there are two relevant representations for a molecule with icosahedral symmetry, and the chemical exchange processes in such a molecule is determined by only two rate parameters. These two rate parameters correspond to the $C_{5}$ and $C_{3}$ rotations of the icosahedral group.

In order to calculate the effect of the chemical exchange on the icosahedral molecules using Eq.(5.50), we need to calculate the coupling coefficients, $a_{m, \mu}^{l, \lambda}$, of Eq.(5.51), and we need to decompose the Wigner rotation matrix elements, $\mathcal{D}^{(l)}$, which forms the irreducible representations of $S O(3)$ group, into the irreducible representations of the icosahedron group I. In order to use Eq.(5.53), we will need the character of the $S O(3)$ group which is given by

$$
\chi^{(l)}(\phi)=\frac{\sin \left(l+\frac{1}{2} \phi\right)}{\sin (\phi)}
$$

Since the icosahedral group contains only rotations through angles of $\frac{2 \pi}{n}$, where $n$ is 
Table 5.7: Characters of I Group

\begin{tabular}{|c|ccccc|}
\hline \hline$I$ & $E$ & $12 C_{5}$ & $12 C_{5}^{2}$ & $20 C_{3}$ & $15 C_{2}$ \\
\hline$A$ & 1 & 1 & 1 & 1 & 1 \\
$T_{1}$ & 1 & $\frac{1+\sqrt{5}}{2}$ & $\frac{1-\sqrt{5}}{2}$ & 0 & -1 \\
$T_{2}$ & 1 & $\frac{1-\sqrt{5}}{2}$ & $\frac{1+\sqrt{5}}{2}$ & 0 & -1 \\
$G$ & 4 & -1 & -1 & 1 & 0 \\
$H$ & 5 & 0 & 0 & -1 & 1 \\
\hline
\end{tabular}

an integer, there is a periodic property of the characters $\chi^{(l)}\left(\frac{2 \pi}{n}\right)$.

$$
\chi^{(l)}\left(\frac{2 \pi}{n}\right)=-\chi^{(n-l-1)}\left(\frac{2 \pi}{n}\right)=-\chi^{(m n-l-1)}\left(\frac{2 \pi}{n}\right),
$$

where $m$ is also an integer. Let us assume that the common multiple is $\mathrm{N}$ for all the rotations in the icosahedral group $I$. When $l=N$, one character will be the sum of the identity representation, $\chi(E)=g=60$, the order of the icosahedral group $G$, and all other characters will be zero. This corresponds to the regular representation. For $l=N m$, we obtain the regular representation $m$ times plus the identity representation. For $1<k<N$, we have

$$
\Gamma^{(N m+k)}=m \Gamma_{\text {reg }}+\Gamma^{(k)}
$$

Furthermore, from Eq.(5.79), it can be easily proved that

$$
\Gamma^{(\dot{k})}+\Gamma^{(N-k)}=\Gamma_{r e g}
$$

where $\Gamma^{(k)}$ is the $k$-th representation of the $S O(3)$, given by the linear combination of the irreducible representations of the icosahedral group. For this reason we only need to tabulate the reduction of the representations of $S O(3)$ group, $\mathcal{D}^{(l)}$, into the irreducible representations of $I$ through $l=14$. In this situation, $N=30$ and the regular representation is given by $\Gamma_{\text {reg }}=A+3 T_{1}+3 T_{2}+4 G+5 H$. 
Table 5.8: Reduction of $\mathcal{D}^{(l)}$ to irreducible representations of $I$

\begin{tabular}{|c|c|}
\hline \hline$l$ & $I$ \\
\hline 0 & 4 \\
1 & $T_{1}$ \\
2 & $H$ \\
4 & $T_{2}+G$ \\
5 & $G+H$ \\
6 & $T_{1}+T_{2}+H$ \\
7 & $A+T_{1}+G+H$ \\
8 & $T_{1}+T_{2}+G+H$ \\
9 & $T_{2}+G+2 H$ \\
10 & $T_{1}+T_{2}+2 G+H$ \\
11 & $A+T_{1}+T_{2}+G+2 H$ \\
12 & $2 T_{1}+T_{2}+G+2 H$ \\
13 & $A+T_{1}+T_{2}+2 G+2 H$ \\
14 & $T_{1}+2 T_{2}+2 G+2 H$ \\
30 & $T_{1}+T_{2}+2 G+3 H$ \\
\hline
\end{tabular}

If we only consider the case in which the asymmetry parameter of the CSA tensor is zero and the local symmetry is therefore determined by the dihedral group $D_{\infty}$, the NMR lineshape of a molecule with icosahedral symmetry under a chemical exchange process is determined only by one rate parameter. We assume that this rate parameter corresponds to the jumps generated by the $C_{5}$ rotations in icosahedral group since the $C_{5}$ rotations require the minimum energy, and therefore are the most favorable jumps in the chemical exchange process. The chemical exchange therefore satisfies strong collision condition and its lineshape can be calculated by 
use of Eq.(5.18).

Table 5.9: Decomposition of the representations of the group $I$ under its subgroup $D_{5}, D_{3}$, and $D_{2}$

\begin{tabular}{|c|ccc|}
\hline \hline$I$ & $D_{5}$ & $D_{3}$ & $D_{2}$ \\
\hline$A$ & $A_{1}$ & $A_{1}$ & $A_{1}$ \\
$T_{1}$ & $A_{2}+E_{1}$ & $A_{2}+E$ & $B_{1}+B_{2}+B_{3}$ \\
$T_{2}$ & $A_{2}+E_{2}$ & $A_{2}+E$ & $B_{1}+B_{2}+B_{3}$ \\
$G$ & $E_{1}+E_{2}$ & $A_{1}+A_{2}+E$ & $A_{1}+B_{1}+B_{2}+B_{3}$ \\
$H$ & $A_{1}+E_{1}+E_{2}$ & $A_{1}+2 E$ & $2 A_{1}+B_{1}+B_{2}+B_{3}$ \\
\hline
\end{tabular}

The first simulation shows the lineshape of an icosahedral molecule in the strong collision limit with an axially symmetric CSA tensor. This situation corresponds to that the local symmetry of the CSA tensor is $D_{\infty}$. Since the local symmetry group has to a subgroup of the icosahedral group in order to find the relevant representations, we choose the largest subgroup of the icosahedral group, $D_{5}$, as the local symmetry group. From Table (5.9), we can know that there is only one relevant representation. Thus, the whole dynamics is determined by a single rate parameter $\kappa$. Figure (5.7) shows a variation of the lineshapes with different chemical exchange rates. At a slow exchange rate, there are three singularities in the lineshape excluding the original two singularities which form the two edges of the static lineshape, two of them are at the left side of the isotropic resonance frequency, and the other one is at right side. The positions of all singularities depend on the exchange rate. Under fast exchange, all sites are degenerate and the spectrum shows a single sharp line at the isotropic resonance frequency. In order to understand these singularities, we choose one of the $12 C_{5}$ rotation axes as the $Z_{M}$ axis and another two of the $15 C_{2}$ rotation axes as the $X_{M}$ and $Y_{M}$ axes. With these definitions, one of the two singularities on the left side of the powder pattern corresponds to the orientation of the icosahedron where 


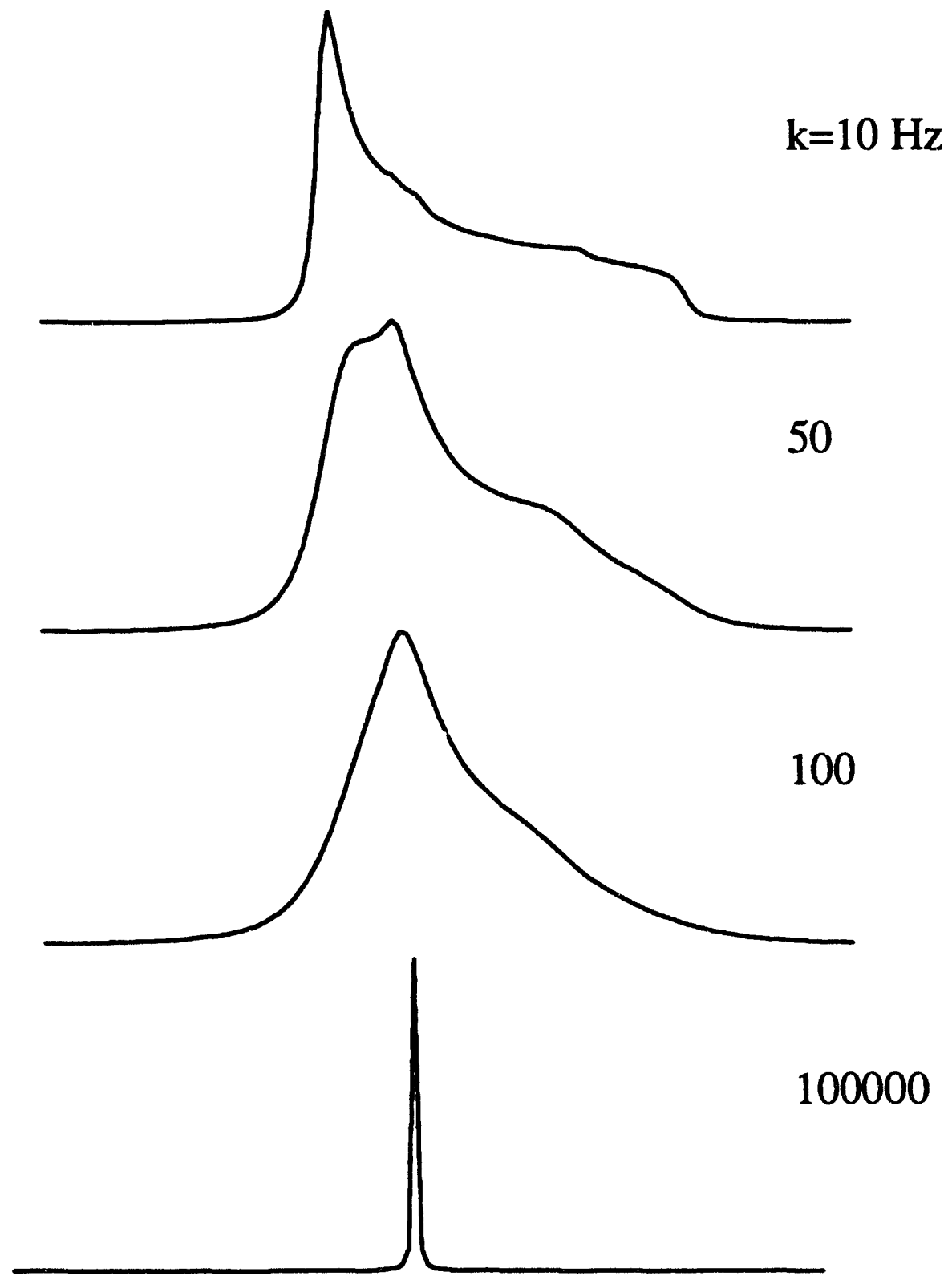

Figure 5.7: Variation of lineshapes with different exchange rates in icosahedron jumps with strong collision limit. The exchange rate is in unit of Hertz; the anisotropy of the CSA tensor is $\omega_{0} \delta=3 k H z$ and its asymmetry parameter is zero. 
the $Z_{M}$ axis is along the external magnetic field. The other one on the left side is generated by the configuration in which the $Z_{M}$ axis is at an angle of $37.38^{\circ}$ with respect to the external field, that is, the external field coincides with one of the $C_{3}$ rotation axes. The third configuration which generates singularities is at the position at which the external field coincides with one of the $C_{2}$ rotation axes. In the first two configurations, all twelve sites degenerate into two different frequencies. Of course, the values and site numbers corresponding to these two set frequencies are different. In the third configuration there three different frequencies. All the frequencies and number of sites with a particular frequency are tabulated in Table (5.10).

Table 5.10: Orientations at which there are some degree of degeneracy of the resonance frequencies of sites in an icosahedral molecule.

\begin{tabular}{|c|c|c|c|c|c|c|c|c|c|}
\hline \hline & $\begin{array}{c}\theta_{1} \\
(\text { degree })\end{array}$ & $\begin{array}{c}\theta_{2} \\
(\text { degree })\end{array}$ & $\begin{array}{c}\theta_{3} \\
(\text { degree })\end{array}$ & $\begin{array}{c}\omega_{1} \\
(\delta)\end{array}$ & $\begin{array}{c}\omega_{2} \\
(\delta)\end{array}$ & $\begin{array}{c}\omega_{3} \\
(\delta)\end{array}$ & $N_{1}$ & $N_{2}$ & $N_{3}$ \\
\hline 1 & 0 & 63.43 & - & 1 & $-\frac{1}{5}$ & - & 2 & 10 & - \\
\hline 2 & $37.38 c$ & 79.19 & - & $\frac{1}{\sqrt{5}}$ & $-\frac{1}{\sqrt{5}}$ & - & 6 & 6 & - \\
\hline 3 & 31.72 & 58.28 & 90 & $\frac{\sqrt{5}+3}{4 \sqrt{5}}$ & $\frac{\sqrt{5}-3}{4 \sqrt{5}}$ & $-\frac{1}{2}$ & 4 & 4 & 4 \\
\hline
\end{tabular}

Now we consider the chemical exchange in the first two configurations in an icosahedral molecule. In general, we can assume that there are $N$ different sites in the molecule but only two distinguishable frequencies in the spectrum: the first $N_{1}$ sites have frequency $\omega_{c_{1}}$ and the other $N_{2}$ sites have another frequency $\omega_{c_{2}}$. We will calculate the eigenvalues of the matrix $\imath \hat{\omega}\left(\Omega_{M}\right)+\hat{\pi}$ instead of exactly solving the dynamical equation for such a system in order to obtain the analytical solution for the singularity. As was done with tetrahedral jump case in the last section, we first define

$$
\begin{aligned}
& \lambda_{1}=\lambda-\imath \omega_{c_{1}}+(N-1) \kappa \\
& \lambda_{2}=\lambda-\imath \omega_{c_{2}}+(N-1) \kappa .
\end{aligned}
$$


The secular equation for eigenvalues $\lambda$ of the matrix $\imath \hat{\omega}\left(\Omega_{M}\right)+\hat{\pi}$ becomes

$$
\begin{aligned}
& \left|\begin{array}{ccccccc}
\lambda_{1} & \kappa & \cdots & \kappa & \kappa & \cdots & \kappa \\
\kappa & \lambda_{1} & \cdots & \kappa & \kappa & \cdots & \kappa \\
\vdots & \vdots & \vdots & \vdots & \vdots & \vdots & \vdots \\
\kappa & \kappa & \cdots & \lambda_{1} & \kappa & \cdots & \kappa \\
\kappa & \kappa & \cdots & \kappa & \lambda_{2} & \cdots & \kappa \\
\vdots & \vdots & \vdots & \vdots & \vdots & \vdots & \vdots \\
\kappa & \kappa & \cdots & \kappa & \kappa & \cdots & \lambda_{2}
\end{array}\right| \\
= & \left(\lambda_{1}-\kappa\right)^{N_{1}-1}\left(\lambda_{2}-\kappa\right)^{N_{2}-1}\left[\lambda_{1} \lambda_{2}+\left(N_{1}-1\right) \kappa \lambda_{1}+\left(N_{2}-1\right) \kappa \lambda_{2}-\left(N_{1}+N_{2}-1\right) \kappa^{2}\right] \\
= & 0 .
\end{aligned}
$$

The solutions of Eq.(5.83) into which Eq.(5.82) is inserted is also obvious: the first two eigenvalues are

$$
\lambda_{1,2}=\frac{\imath\left(\omega_{c_{1}}+\omega_{c_{2}}\right)+N \kappa \pm \sqrt{\left[N \kappa-\imath\left(\omega_{c_{1}}+\omega_{c_{2}}\right)\right]^{2}+4\left[\omega_{c_{1}} \omega_{c_{2}}+\imath\left(\omega_{c_{1}} N_{1}+\omega_{c_{2}} N_{2}\right) \kappa\right]}}{2}
$$

Next $N_{1}-1$ eigenvalues are degenerate and they have a value $\lambda_{i}=\imath \omega_{c_{1}}-N \kappa$ for $i=3, \cdots, N_{1}+1$. The final $N_{2}-1$ eigenvalues are also degenerate with the value $\lambda_{i}=w_{c_{2}}-N \kappa$ for $i=N_{1}+2, \cdots, N$. Obviously for these $N-2$ eigenvalues, the frequencies corresponding to the imaginary parts of the eigenvalues do not change with the variation of the exchange rate $\kappa$, but the linewidths of the peaks increase as the exponential of $-N \kappa$. On the other hand, for the first two eigenvalues, the behavior is similar to the two site jump. The only difference is that once total number of sites are larger than two there is a imaginary part in the square root. This imaginary part introduces an additional phase factor which mixes the resonance frequencies with the exchange rate $\kappa$,

$$
\begin{aligned}
\omega_{1,2} & =\frac{1}{2}\left(\omega_{c_{1}}+\omega_{c_{2}} \mp \sqrt{C} \sin \frac{\theta}{2}\right) \\
\kappa_{1,2} & =\frac{1}{2}\left(-N \kappa \pm \sqrt{C} \cos \frac{\theta}{2}\right),
\end{aligned}
$$


where

$$
\begin{aligned}
C & =\sqrt{A^{2}+B^{2}} \\
\theta & =\tan ^{-1} \frac{B}{A} \\
A & =\left[(N \kappa)^{2}-\left(\omega_{c_{1}}-\omega_{c_{2}}\right)^{2}\right] \\
B & =2 \kappa\left[N\left(\omega_{c_{1}}+\omega_{c_{2}}\right)-2\left(N_{1} \omega_{c_{1}}+N_{2} \omega_{c_{2}}\right)\right]
\end{aligned}
$$

Eq.(5.83) can be generalized to the situation in whicl there are only $M$ different frequencies in a molecule with $N(\geq M)$ different sites. Now we define

$$
\lambda_{i}=\imath \omega_{i}-(N-1) \kappa-\lambda, \quad \text { for } i=1,2, \cdots, M
$$

where $\omega_{i}$ is the $i$ th frequency. There are $N_{i}$ such sites in the molecule. The solution of the secular equation for the matrix $\imath \hat{\omega}\left(\Omega_{M}\right)+\hat{\pi}$ is

$$
\operatorname{det}\left[\imath \hat{\omega}\left(\Omega_{M}\right)+\hat{\pi}\right]=\prod_{i=1}^{M}\left(\lambda_{i}-\kappa\right)^{N_{i}-1}\left(\lambda_{i}^{i-1}-\left(N_{i}-1\right) \kappa^{i-1}\right),
$$

where

$$
\begin{aligned}
\lambda_{i}^{i} & =\lambda_{i}^{i-1}-\frac{N_{i-1}\left(\kappa^{i-1}\right)^{2}}{\lambda_{i-1}^{i-1}+\left(N_{i-1}-1\right) \kappa^{i-1}} \\
\kappa^{i} & =\frac{\left(\lambda_{i-1}^{i-1}-\kappa^{i-1}\right) \kappa^{i-1}}{\lambda_{i-1}^{i-1}+\left(N_{i-1}-1\right) \kappa^{i-1}}
\end{aligned}
$$

with

$$
\begin{aligned}
\lambda_{i}^{0} & =\lambda_{i} \\
\kappa^{0} & =\kappa .
\end{aligned}
$$

The first $M$ eigenvalues can only be obtained by solving an $M$ order polynomial equation. The other $N-M$ eigenvalues are

$$
\lambda=\omega_{i}-N \kappa, \quad \text { for } i=M+1, \cdots, N \text {. }
$$

For example, when $M=3$, we assume that the three different frequencies are $\omega_{1}, \omega_{2}$, $\omega_{3}$ corresponding to $N_{1}, N_{2}$, and $N_{3}$ sites respectively. The first three eigenvalues 
therefore are determined by a cubic equation.

$$
\lambda^{3}-c_{1} \lambda^{2}-c_{2} \lambda-c_{3}=0
$$

where

$$
\begin{aligned}
c_{1}= & \omega_{1}+\omega_{2}+\omega_{3}-2 N \kappa \\
c_{2}= & N \kappa\left(\omega_{1}+\omega_{2}+\omega_{3}\right)-(N \kappa)^{2}+\kappa\left(N_{1} \omega_{1}+N_{2} \omega_{2}+N_{3} \omega_{3}\right) \\
& -\left(\omega_{1} \omega_{2}+\omega_{2} \omega_{3}+\omega_{1} \omega_{3}\right) \\
c_{3}= & N \kappa^{2}\left(N_{1} \omega_{1}+N_{2} \omega_{2}+N_{3} \omega_{3}\right)+\omega_{1} \omega_{2} \omega_{3} \\
& -\kappa\left[\left(N_{1}+N_{2}\right) \omega_{1} \omega_{2}+\left(N_{2}+N_{3}\right) \omega_{2} \omega_{3}+\left(N_{1}+N_{3}\right) \omega_{1} \omega_{3}\right] .
\end{aligned}
$$

For the first configuration of an icosahedral molecule shown in Table (5.10), the total number of jumping sites is twelve, $N_{1}=10$ and $N_{2}=2$. After these numbers are inserted into Eq.(5.86), the variation of the frequencies and the linewidths with the chemical exchange rate $\kappa$ was calculated and the results are shown in Figure (5.8). For the first eigenvalue, the resonance frequency decreases from $\delta$ to $0.8 \delta$ while the exponent of the linewidth $\kappa_{1}$ increases according to $-12 \kappa$, obtained from Eq. (5.91) with $N=12$. In other words, one of the edges of the static lineshape will be smooths immediately as the exchange rate increases from zero. For the second eigenvalue, the behavior is quite different. At $\kappa=0$, the resonance frequency is $-\frac{1}{5} \delta$. When the exchange rate increases to the value of $0.4 \delta$, the resonance frequency shifts almost to zero. The exponent of the linewidth changes even more dramatically. At very small exchange rate, it increases with $\kappa$ and reaches the maximum value at around $\kappa=\frac{\delta}{10}$, then decreases with $\kappa$ to zero. Obviously this point forms a singularity in the powder lineshape.

For the second configuration of the icosahedral molecule, inserting the numbers in Table (5.10) into Eq. (5.86) yields the variation of the frequencies, $\omega_{i}$, and the exponential factors, $\kappa_{i}$, responsible for the linewidths with the chemical exchange 

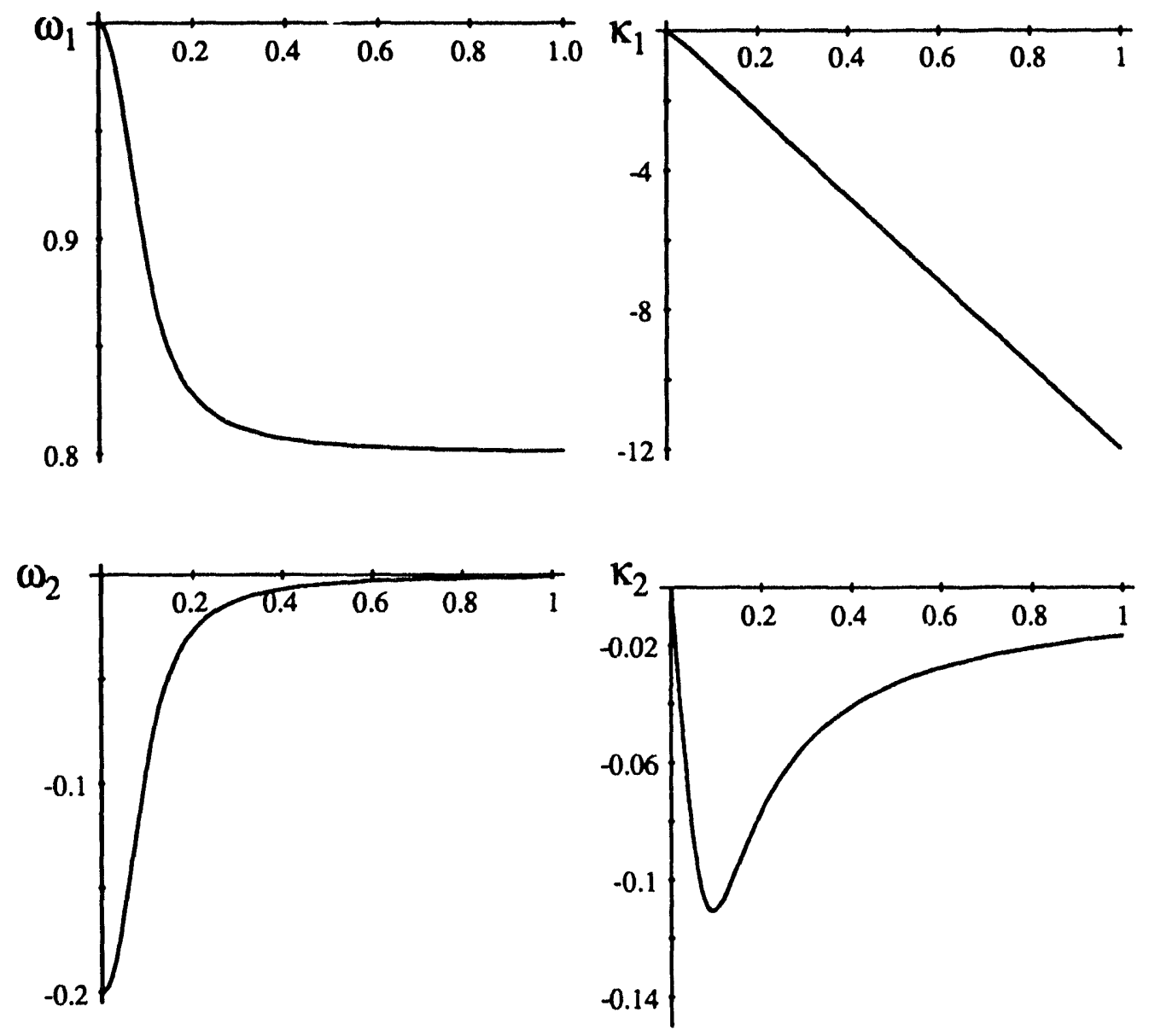

Figure 5.8: Variations of the first two eigenvalues of the matrix $\imath \hat{\omega}+\hat{\pi}$ with the chemical exchange rate $\kappa$ in an icosahedral molecule whose $Z_{M}$ axis (one of the $C_{5}$ rotation axes) is along the external magnetic field and therefore $\theta_{1}=0^{\circ}, \theta_{2}=63.43^{\circ}$, $\omega_{1}=\delta, \omega_{2}=-\frac{1}{5} \delta, N_{1}=2$ and $N_{2}=10$ with $\delta=1$. Left: frequencies, and right: line broadening factors. 
rate $\kappa$. This variation is graphically shown in Figure (5.9). The behavior of the eigenvalues in this figure are similar to the first case. However, there is one obviously discontinuous point at $\kappa=\frac{3}{4} \delta$. At this point, the frequencies of the two eigenvalues are zero exactly and one of the exponential factors, $\kappa_{2}$, responsible for the linewidths changes its variation from increasing to decreasing with the exchange rate, $\kappa$, at this point too. Before this point, the exponential factor $\kappa_{2}$ increases according to $-6 \kappa$ with the exchange rate $\kappa$. The other exponential factor of the linewidth is still roughly increases according to $12 \kappa$ with the exchange rate $\kappa$ except there are a slight changes in very small exchange rate region.

In the third configuration, the variation of the eigenvalues with the chemical exchange rate $\kappa$ is shown in Figure (5.10), resulting from Eqs.(5.92) and (5.93) into which the numbers listed in the third row of Table (5.10) are inserted. The first and third eigenvalues vary with the exchange rate $\kappa$ in manner similar to the above two cases. The resonance frequency of the first eigenvalue decreases from $\frac{\sqrt{5}+3}{4 \sqrt{5}} \delta$ to about $0.3 \delta$, while the frequency of the third eigenvalue increases from $-\frac{1}{2} \delta$ to about $-0.3 \delta$ as the exchange rate $\kappa$ varies from zero to infinity. The linewidth of the both peaks however always increase with an exponent of $-12 \kappa$. The resonance frequency of the second eigenvalue first decreases from $\frac{\sqrt{5}-3}{4 \sqrt{5}} \delta$ to about $-0.2 \delta$ and then increases to zero after the turning point, $\kappa=\frac{1}{2} \delta$, as the exchange rate changes from the zero to infinity. Its line broadening factor also first increases according to $6 \kappa$ and then decreases to zero. These features can also be seen in Figure (5.7), the singularity at about $\omega=\frac{1}{2} \delta$ on the right side of the spectra, which corresponds to the first eigenvalue here, is much more broadened than the singularities at left side in the spectra.

At the beginning of this section, we used group theory to prove that chemical exchange in an icosahedral molecule satisfies the strong collision condition. Now we can further examine this conclusion by numerical simulations of the chemical 

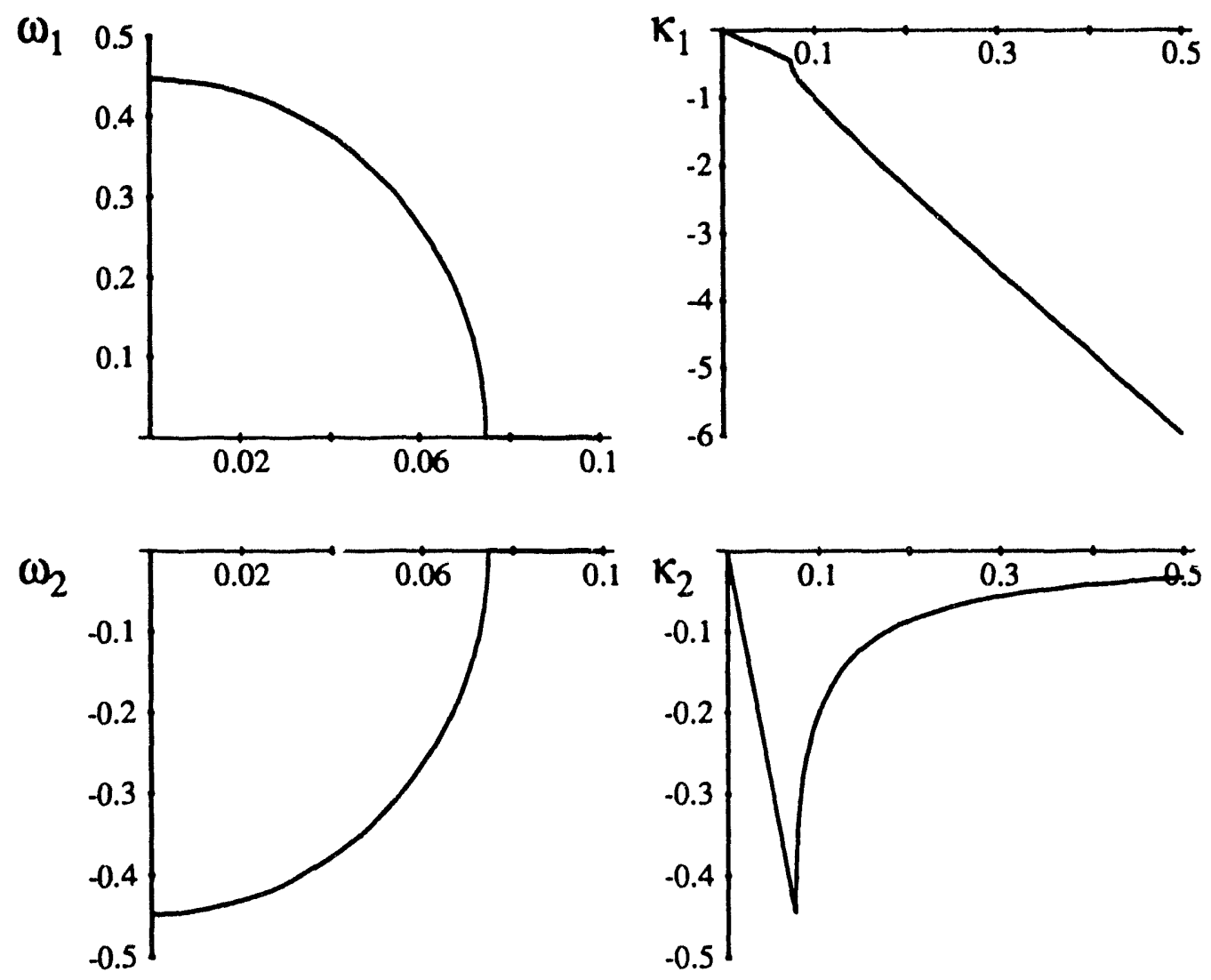

Figure 5.9: Variations of the first two eigenvalues of the matrix $\boldsymbol{\imath}+\hat{\boldsymbol{\pi}}$ with the exchange rate $\kappa$ in an icosahedral molecule. The $Z_{M}$ axis, one of the $C_{3}$ axes, is along the external field and $\theta_{i}, \omega_{i}$, and $N_{i}$ are given in Table (5.10). Left: frequencies, and right: line broadening factors with $\delta=1$. 

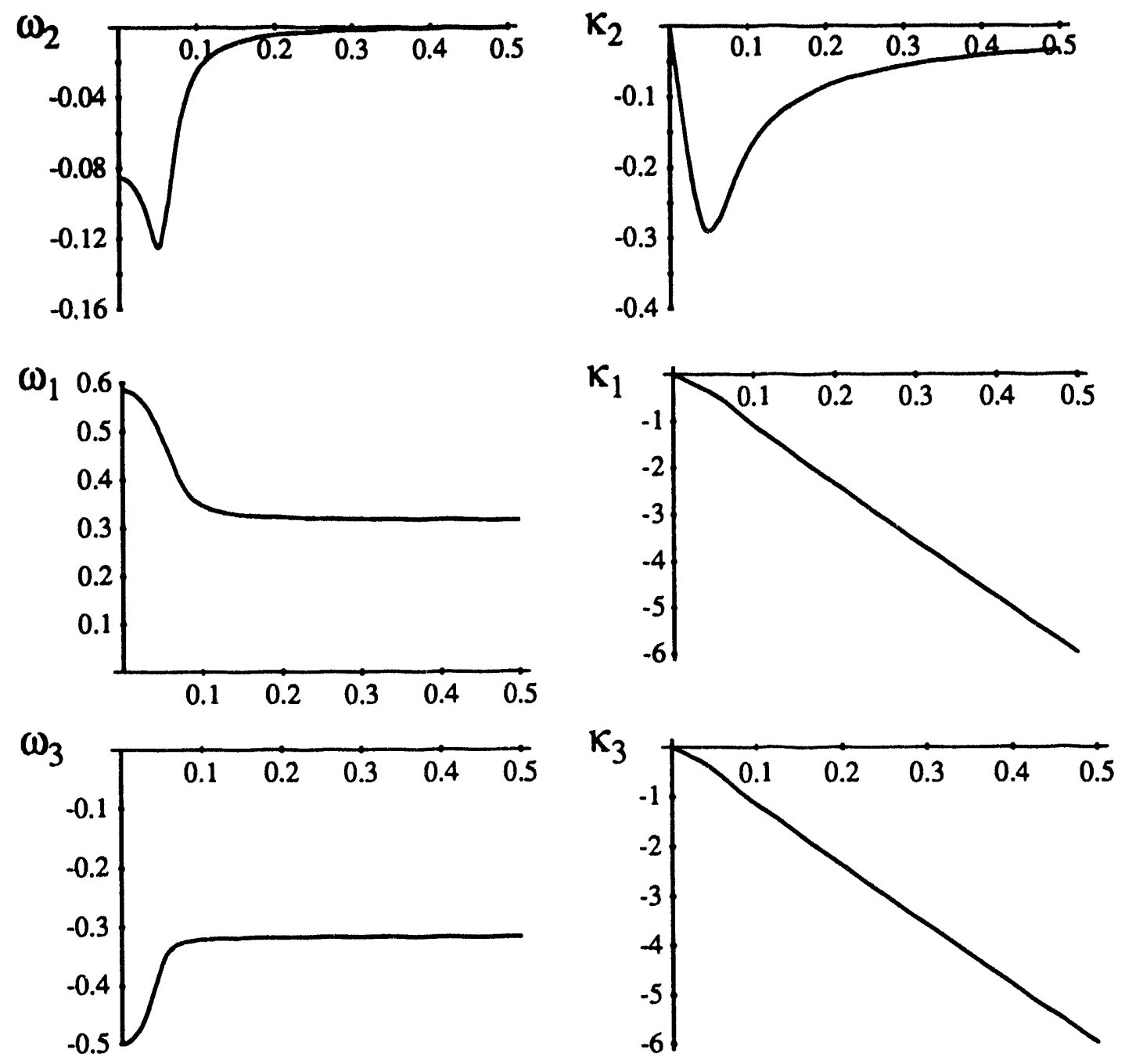

Figure 5.10: Variations of the first two eigenvalues of the matrix $\imath \hat{\boldsymbol{\omega}}+\hat{\pi}$ with the exchange rate $\kappa$ in an icosahedral molecule. The $Z_{M}$ axis, one of the $C_{2}$ axes, is along the external field and $\theta_{i}, \omega_{i}$, and $N_{i}$ are given in Table (5.10). Left: frequencies, and right: line broadening factors with $\delta=1$. 

$\kappa(\mathbf{H z})$
(a) $\mathrm{C}_{3}$
(b) $\mathrm{C}_{5}$
(c) $\mathrm{C}_{5}^{2}$

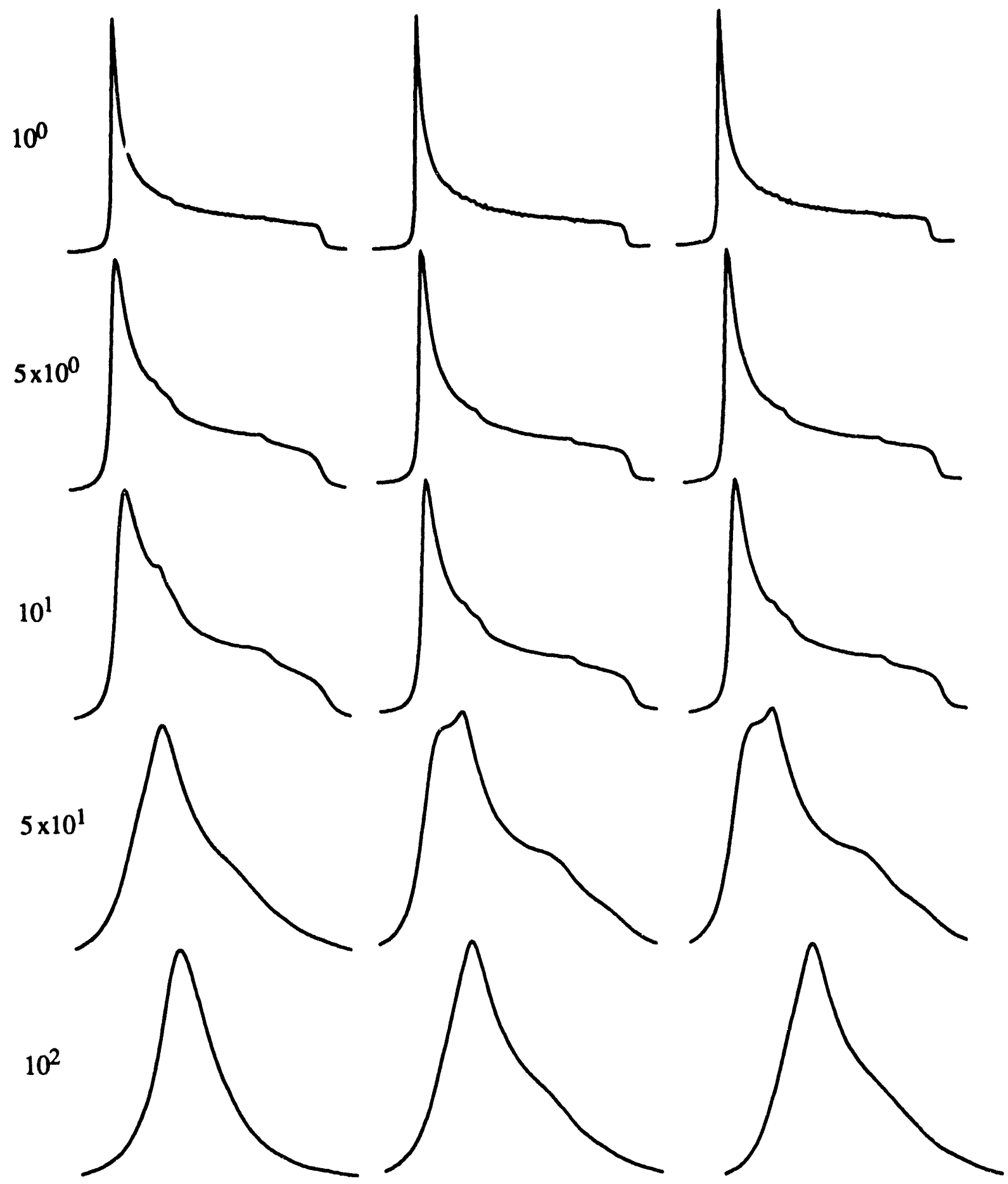

Figure 5.11: Variation of lineshapes with different exchange rates in icosahedron jumps: (a) $C_{3}$ jump mode, (b) $C_{5}$ jump mode, and (c) $C_{5}^{2}$ jump mode. The exchange rate is in unit of Hertz; the anisotropy of the CSA terisor is $\omega_{0} \delta=3 k H z$, and its asymmetry parameter is zero; spectrum width is $S W=6 \mathrm{kHz}$. 
exchange process in the icosahedral molecule in the weak collision limit. If we assume the atoms in the icosahedral molecule are set on the vertices of an icosahedron, the $Z$ axis in the principle axis system of the CSA tensor will coincide with the $C_{b}$ rotation axis. If the asymmetric factor $\eta$ of the CSA tensor is zero, the local symmetry of the CSA tensor, as we know, is determined by $D_{\infty}$. However, there are four physically different classes of rotations. Each class of rotations corresponds to one jumping mode in the icosahedral molecules with a rate parameter $\kappa_{r}$. We assume that these rate parameters independently form the lineshape under the chemical exchange. We can then examine the effects of each individual jumping mode on the lineshape. Figure (5.11) shows variations of the lineshapes with different exchange rates under the weak collision condition and different jumping modes: (a) $C_{3}$ jump mode, (b) $C_{5}$ jump mode, and (c) $C_{5}^{2}$ jump mode. $C_{2}$ jumps are not considered because they require large amounts of energy, and thus do not happen often. All simulated lineshapes are very much same as those obtained in the strong collision limit. The only observable difference between the lineshapes in the strong and weak collision limits is that the linewidths of the peaks in the strong collision limit narrow at different speeds with different jumping modes. Such a difference will disappear if we redefine the exchange rate because each jump mode can be implemented by the linear combination of another jump modes. If we properly normalize these difference can be eliminated, and all lineshapes are exactly same as those in the strong collision limit.

Another example is chemical exchange in a dodecahedral molecule. In the dodecahedral molecule, there are twenty sites, each on the vertices of a dodecahedron. The molecule symmetry also has the icosahedral symmetry. The local symmetry of the CSA tensor for each site is determined by the dihedral subgroup, $D_{5}$, of $I$ for the case of $\eta=0$, and, therefore, chemical exchange in a dodecahedral molecule also satisfies the strong collision condition. Figure (5.12) shows the variations of the 


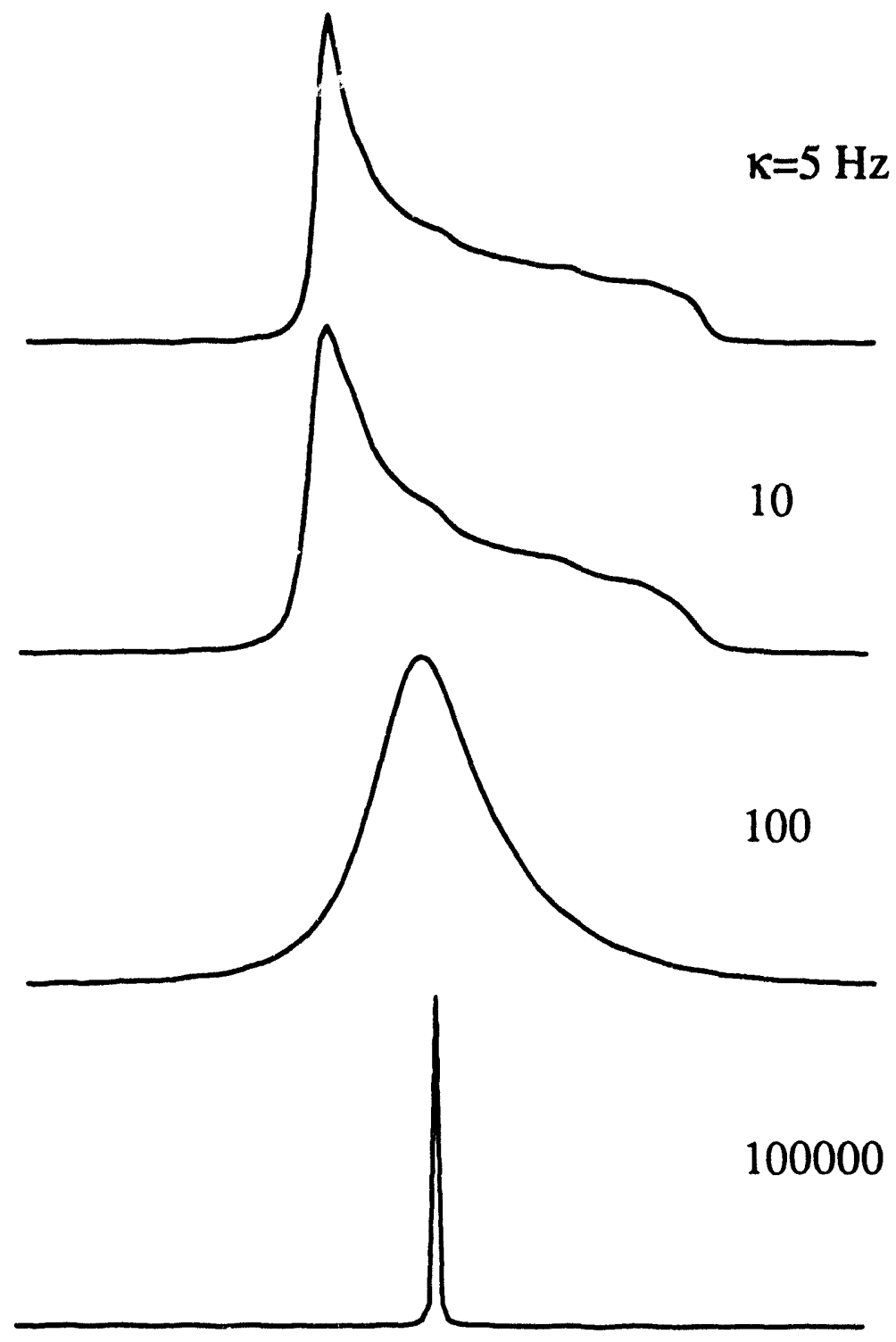

Figure 5.12: Variation of lineshapes with different exchange rates in dodecahedron jumps with strong collision limit. The exchange rate is in unit of Hertz; the anisotropy of the CSA tensor is $\omega_{0} \delta=3 k H z$ and its asymmetry parameter is zero; the spectrum width is $s w=10 \mathrm{kHz}$. 
lineshapes in a dodecahedral molecule with different exchange rates. At slow rates, three singularities are clearly seen in the figure. The positions of the singularities are more or less same as those in the lineshape of an icosahedral molecule. One difference is that the overall linewidth decreases much faster than in the icosahedral case. These singularities correspond to three different configurations of the dodecahedral molecule relative to the external magnetic field. In the first configuration, one of the $C_{5}$ rotation axis coincides with the external field, and all twenty sites form two different groups. Each group has ten equivalent sites and a frequency $\omega_{i}$ for $i=1,2$. In the second configuration, one of the $C_{3}$ rotation is along the external field, and therefore the twenty sites are divided into three different groups. The first group with two equivalent sites on the $Z_{M}$ axis has the frequency $\delta$. The second group has six sites in which three of them are equally distributed on a cone with half apex angle of $41.81^{\circ}$ with respect to the external field and the other three sites are on a cone which is the mirror imager of the first cone about the $X_{M} Y_{M}$ plane. The twelve sites left, which form the third group, are distributed on a cone with half apex angle of $70.55^{\circ}$ in a way same as the second group. In the third configuration, in which one of the $C_{2}$ rotation axes aligns on the external field, there are four different groups. The first group forms a cone with the half apex angel $20.09^{\circ}$, and the second group with $54.74^{\circ}$, the third group with $58.28^{\circ}$ and the fourth group are distributed on the equator. All geometrical and magnetic properties of the three configurations of the dodecahedral molecule are summarized in the Table (5.11).

Figure (5.13) shows variations of the lineshapes in a dodecahedron molecule with different exchange rates under the weak collision condition and different jumping modes. (a) corresponds to $C_{3}$ jump mode, (b) $C_{5}$ jump mode, and (c) $C_{5}^{2}$ jump mode. The lineshape variations with different jump modes are similar to those in icosahedral molecules. The difference of the linewidths arising from different jump modes will be disappears if we redefine the jumping rate for each jump mode. 


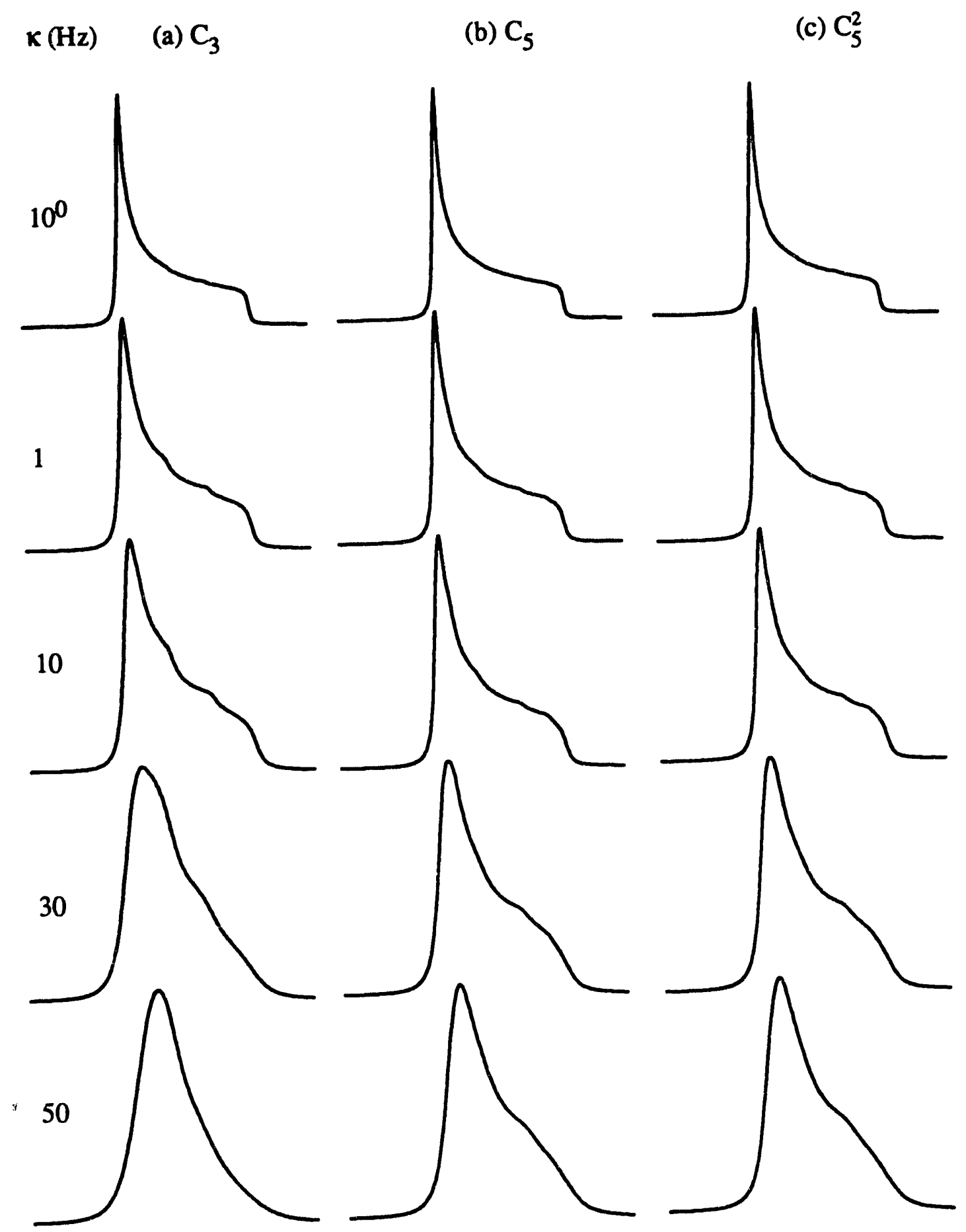

Figure 5.13: Variation of lineshapes with different exchange rates in dodecahedron jumps with weak collision limit: (a) $C_{3}$ jump mode, (b) $C_{5}$ jump mode, and (c) $C_{5}^{2}$ jump mode. The exchange rate is in unit of Hertz; the anisotropy of the CSA tensor is $\omega_{0} \delta=3 k H z$ and its asymmetry parameter is zero. 
Table 5.11: Orientations at which there are some degree of degeneracy of the resonance frequencies of sites in a dodecahedral molecule

\begin{tabular}{|c|c|c|c|c|c|c|c|c|c|c|c|c|}
\hline \hline & $\theta_{1}$ & $\theta_{2}$ & $\theta_{3}$ & $\theta_{4}$ & $\omega_{1}$ & $\omega_{2}$ & $\omega_{3}$ & $\omega_{4}$ & $N_{1}$ & $N_{2}$ & $N_{3}$ & $N_{4}$ \\
\hline \multicolumn{8}{|c|}{ degree } & \multicolumn{8}{|c|}{$(\delta)$} \\
\hline 1 & 37.38 & 79.19 & - & - & $\frac{1}{\sqrt{5}}$ & $\frac{-1}{\sqrt{5}}$ & - & - & 6 & 6 & - & - \\
\hline 2 & 0 & 41.81 & 70.53 & - & 1 & $\frac{1}{3}$ & $\frac{-1}{3}$ & - & 2 & 6 & 12 & - \\
\hline 3 & 20.91 & 54.74 & 58.28 & 90 & $\frac{1+\sqrt{5}}{4}$ & 0 & $\frac{\sqrt{5}-3}{4 \sqrt{5}}$ & $\frac{-1}{2}$ & 4 & 8 & 4 & 4 \\
\hline
\end{tabular}

\subsection{Motions in Solid $C_{60}$}

Study of carbon clusters was ignited in the 1960s by the observation vith microwave spectroscopy of polyyne, $\left(\mathrm{HC}_{5} \mathrm{~N}\right)$, in space by Townes and his co-workers[120]. Since then, many different sizes, up to 190 carbons have been observed [121, 122, 123, 126]. Among these results, the most interesting was shown by Rohlfing and coworkers[126]. They used the carbon cluster-beam method and observed carbon clusters larger than 33-atoms. This experiment resulted in the first experimental observation of the $C_{60}$ molecule. From their spectra, the $C_{60}$ molecule was always produced in greater intensities than the other clusters. Later, Kroto and co-workers[124] repeated Rohlfing's experiment. After applying purifying techniques to the experiment, they observed an even stronger signal of $C_{60}$ clusters in the mass spectra, with the $C_{60}$ cluster eventually dominating the whole spectrum. In order to understand the dominance of the $C_{60}$ in the carbon cluster-beam experiment, Kroto and co-workers thought that the behavior could result from the stabilization by the closure of the graphitic net into a hollow chicken-wire cage similar to the geodesic domes of Buckminster Fuller. This molecule would have a truncated icosahedral structure or a soccer ball type structure (see Figure 5.14)[124].

More recently, success in synthesizing $C_{60}$ molecules in macroscopic amounts[127] has stimulated intense interest and activity. The study of the physical properties 


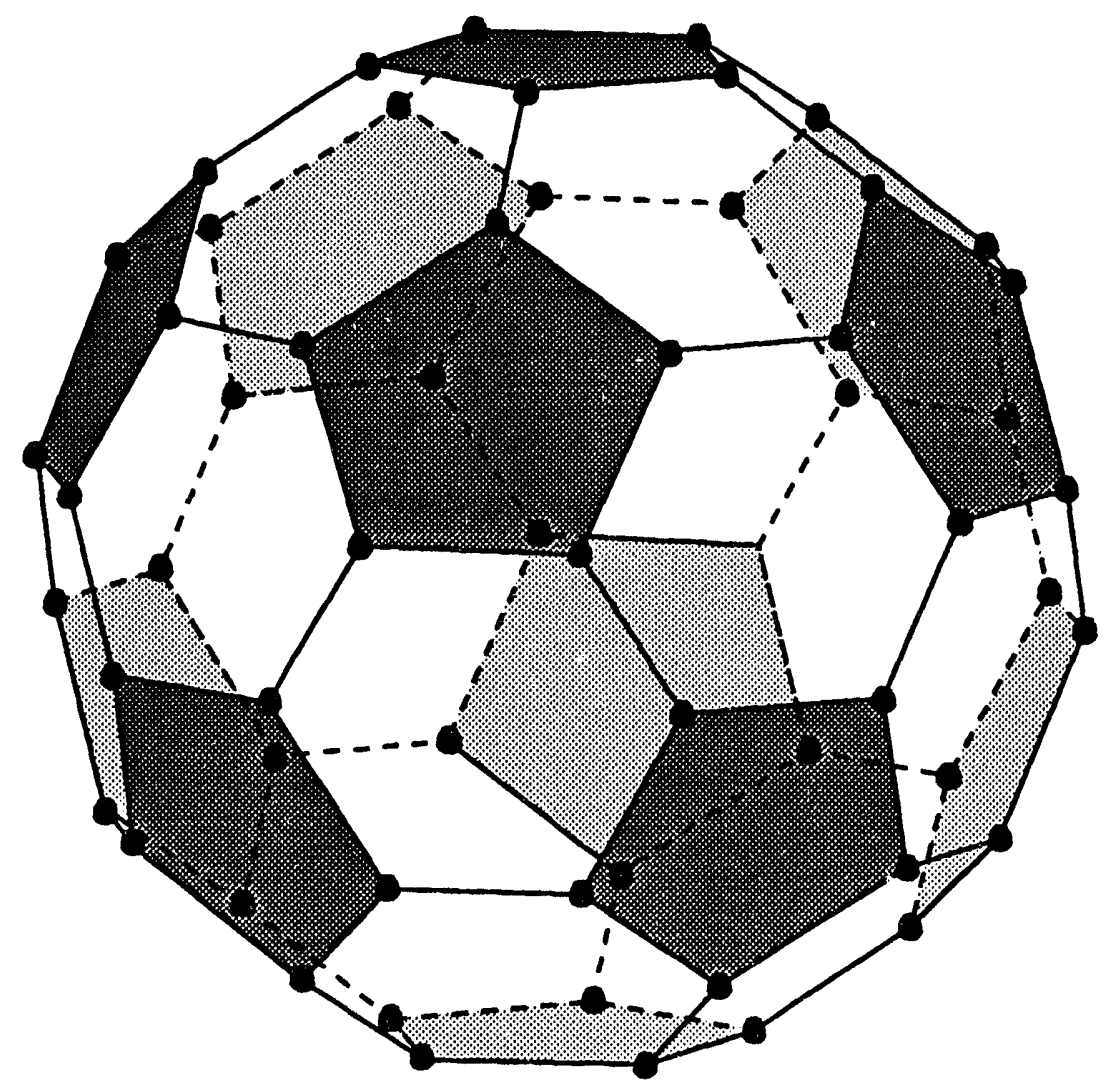

Figure 5.14: A truncated icosahedral structure, or a soccer ball type Buckminster Fuller, of a $C_{60}$ molecule. 
of $C_{60}$ molecules in the solid state has clearly demonstrated its conductivity and superconductivity upon doping with alkali metal atoms (potassium or rubidium) [132, 133]. The crystal structure adopted by $C_{60}$ molecules shows that the mass center of each $C_{60}$ molecule is positioned in a fcc lattice, and the whole molecule behaves like a single large atom. Meanwhile, a number of spectroscopic studies, especially the infrared, vibrational Raman spectra, and liquid NMR spectra, have confirmed that the molecular symmetry is indeed icosahedral $[134,135,136]$.

The pseudospherical symmetry of the $C_{60}$ molecules implies that the motion of the molecules is probably isotropic in the solid state. The solid-state NMR spectroscopy therefore provides a unique probe of this kind of molecular motion. The ${ }^{13} C$ NMR spectra of solid $C_{60}$ have shown unequivocally that each individual $C_{60}$ molecule reorients rapidly and isotropically in the solid state at room temperature $[137,139$, $140,138]$ because there is only one sharp, liquid-like isotropic peak at 143ppm in spectra. As the temperature decreases, the random reorientation process of the molecule will, as expected, slow down and a characteristic CSA powder pattern with principal values of $\sigma_{11}=220 p p m, \sigma_{22}=186 p p m, \sigma_{33}=33 p p m$ at temperature $T=77 \mathrm{~K}$ was obtained. The lineshape of the powder spectrum of $C_{60}$ also changes and the intensity of the sharp peak decreases as the temperature is lowered. Such a result may be explained by the growth of a phase in which cluster rotation is inhibited or by a distribution of rotational correlation times and a concomitant distribution of motional barries.

We have performed numerical simulations by assuming that the $C_{60}$ molecules randomly and discretely jumps on an icosahedron at low temperature. We first assume that the asymmetry parameter of the local CSA tensor is zero. According to the discussion in last section, the local symmetry of the CSA tensor is determined by the $D_{\infty}$ group in which $D_{2}, D_{3}$, and $D_{5}$ are also the subgroups of the icosahedral group. If we choose the highest dihedral group, $D_{5}$, as the local symmetry group, the 


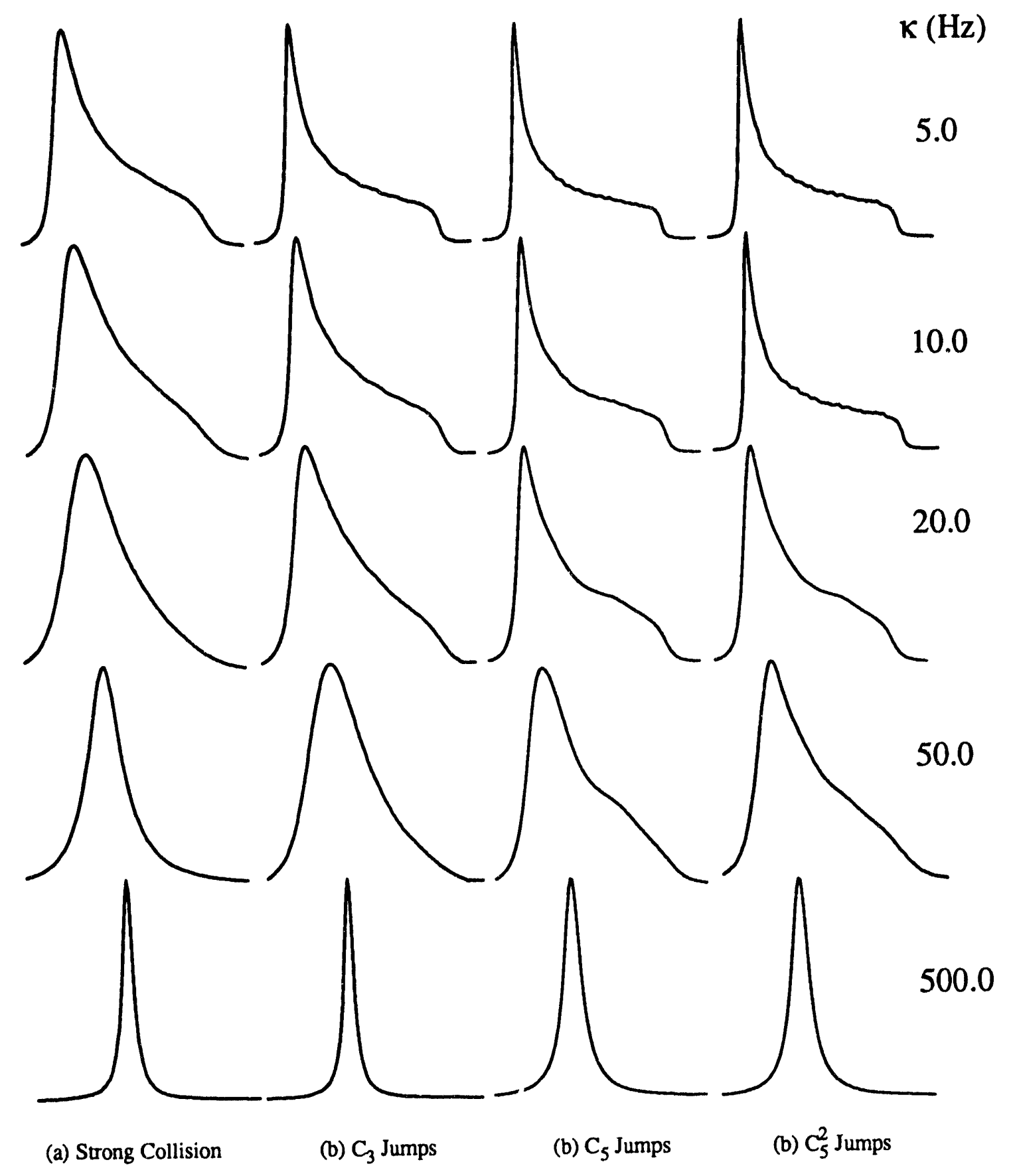

Figure 5.15: Variation of simulated powder lineshapes of $C_{60}$ molecules in the solid state with different jumping rates, $\kappa$, and with different jumping modes in the case that the asymmetry parameter of the CSA tensor is zero, the anisotropy is $\omega_{0} \delta=3 k H z$, and the spectrum width is $6 \mathrm{kHz}$. (a) In the strong collision limit, (b) $C_{3}$ jumps, (c) $C_{5}$ jumps, and (d) $C_{5}^{2}$ jumps in the $C_{60}$ molecules. 


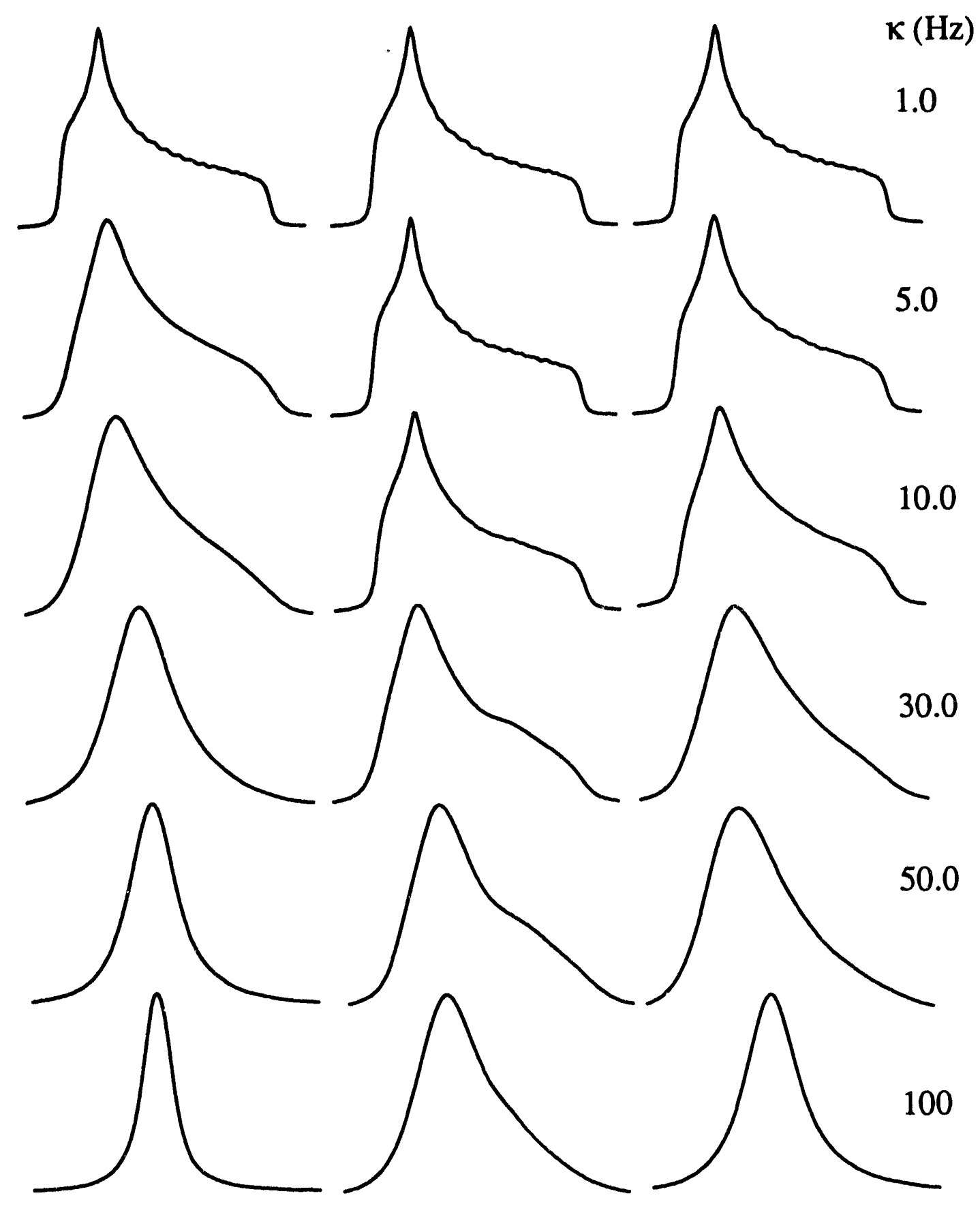

(a) Strong Collision

(b) $\mathrm{C}_{5}$ Jumps

(b) $\mathrm{C}_{3}$ Jumps

Figure 5.16: Variation of simulated powder lineshapes of $C_{60}$ molecules in the solid state with different jumping rates, $\kappa$, and with different jumping modes in the case that the asymmetry parameter of the CSA tensor is 0.312 , obtained from the experimental results given in reference [140], the anisotropy is $\omega_{0} \delta=3 \mathrm{kHz}$, and the spectrum width is $6.8 \mathrm{kHz}$. (a) In the strong collision limit, (b) $C_{5}$ jumps, and (c) $C_{3}$ jumps in the $C_{60}$ molecules. 
number of relevant representations is one from Table (5.9) except the identity representation. The molecular reorientation process therefore satisfies the strong collision condition. Figure (5.15) shows the variations of the simulated powder lineshapes of $C_{60}$ molecules with different exchange rates $\kappa$, using different jumping modes. In Figure (5.15a), the spectra are calculated in the strong collision model while in Figure $(5.15 \mathrm{~b}),(5.15 \mathrm{c})$, and $(5.15 \mathrm{~d})$, by assuming that the $C_{60}$ molecules undergo random jumps around the $C_{3}, C_{5}$, and $C_{5}^{2}$ rotation axes in the weak collision limit respectively. The lineshapes in the strong collision limit narrow the fastest, and those in the $C_{5}$ and $C_{5}^{2}$ jumping modes narrow the slowest. This difference will disappear if we redefine the jumping rate. The lineshapes in the $C_{5}$ jumping mode are exact same as those in the $C_{5}^{2}$ jumping mode.

Figure (5.16) shows the variations of the lineshapes of the $C_{60}$ molecules in the solid state with different jumping rates, using different jumping modes for the case that the asymmetry ? arameter of the CSA tensor is $\eta=0.312$, obtained from the experimental results given in reference [140]. As can be seen, the variations of the lineshapes are similar to those shown in Figure (5.15). The linewidth of the lineshape arising from the strong collision limit narrows the fastest, and then the one with $C_{3}$ jump mode while the linewidth narrows the slowest with $C_{5}$ jump mode. The lineshapes vary largely same. This result seems in contrast to the conclusion from the group theoretical discussion which says that two exchange rate parameters are necessary to determine the lineshape during chemical exchange if the local symmetry of the CSA tensor is $D_{2}$. One reason could be that the asymmetry parameter is still too small to see the difference of the lineshape between different jump modes. From these simulated powder lineshapes with chemical exchange, two things may be concluded. First, the rate change is very small from the rigid static structure to the state in which the molecules tumble isotropically and locally. This means that the molecules require a very small amount of kinetic energy to be in the isotropically tumbling state. In this state, the crystal structure may still be well defined because the molecules only tumble locally. This may explains the difference between the NMR and $x$-ray diffraction results. The NMR results showed that the spectra at 
the temperature above $200 \mathrm{~K}$ contain only an isotropic narrow peak while the $\mathrm{x}$ ray results indicated a phase transition at about $250 \mathrm{~K}$ in solid $C_{60}[141]$. Secondly, because the powder lineshape changes smoothly and there is no singularity in the spectra as the exchange rate changes, the conclusion given by Tycko and Yannoni and co-workers are consistent. During the process of lowering temperature, there is either a phase in which the carbon cluster rotation is inhibited or a distribution of the rotational correlation times. These conclusions are further supported by the NMR relaxation measurement of $C_{60}$ in the solid state by Tycko and co-workers[138]. 


\section{Bibliography}

[1] N. Bloembergen and J.A. Rowland, Acta. Met. 1, 173 (1963).

[2] F. Reif and E.M. Purcell, Phys. Rev., 91, 631 (1953).

[3] T.P. Das and E.L. Hahn, Sol. State, Phys. suppl. 1 (1958).

[4] A. Abragam and W.G. Proctor, Phys. Rev., 109, 1441 (1958).

[5] A. Abragam, Principles of Nuclear Magnetism, (Clarendon, Oxford, 1982).

[6] M. Goldman, Spen Temperature and Nuclear Magnetic Resonance in Solids, (Clarendon, Oxford, 1970).

[7] R.V. Pound, Phys. Rev., 81, 278 (1951).

[8] D.P. Weitekamp, A. Bielecki, D. Zax, K. Zilm and A. Pines, Phys. Rev. Lett., 50, 1807 (1983).

[9] D. Zax, A. Bielecki, K.W. Zilm, A. Pines, and D.P. Weitekamp, J. Chem. Phys., 83, 4877 (1985).

[10] R. Kreis, D. Suter, and R.R. Ernst, Chem. Phys. Lett., 118, 120 (1985).

[11] A.M. Thayer and A. Pines, Acc. Chem. Res., 20, 47 (1987).

[12] J.M. Millar, A.M. Thayer, A. Bielcki, D.B. Zax, and A. Pines, J. Chem. Phys., 83, 934 (1985).

[13] A.M. Thayer and A. Pines, J. Magn. Reson., 70, 518 (1986).

[14] , M. Luzar, A.M. Thayer, and A. Pines, Mol. Phys., ? (1987). 
[15] T.P. Jarvie, A.M. Thayer, J.M. Millar, and A. Pines, J. Phys. Chem., 91, 2240 (1987).

[16] A.M. Thayer, J.M. Millar, and A. Pines, Chem. Phys. Lett., 129, 55 (1986).

[17] D. Suter, T.P. Jarvie, B.-Q. Sun and A. Pines, Phys. Rev. Lett., 59, 106 (1988).

[18] B.-Q. Sun, S. Matsui, D. Suter, and A. Pines, in preparation.

[19] M. Mehring, Principles of High Resolution NMR in Solids, 2nd ed. (SpringerVerlag, Berlin, 1983).

[20] M. E. Rose, Elementary Theory of Angular Momentum, (John Wiley \& Sons, New York, 1957).

[21] G.J. Bowden and W.D. Hutchison, J. Magn. Reson., 67, 403 (1986).

[22] G.J. Bowden and W.D. Hutchison, J. Magn. Reson., 67, 415 (1986).

[23] G.J. Bowden and W.D. Hutchison, J. Magn. Reson., 70, 361 (1986).

[24] G.J. Bowden and W.D. Hutchison, J. Magn. Reson., 72, 61 (1986).

[25] D.P. Weitekamp, Adv. Magn. Reson., 11, 111 (1983).

[26] G. Bodenhausen, Prog. in NMR Spectrosc., 14, 137 (1981).

[27] G. Drobny, D.P. Weitekamp, and A. Pines, Chem. Phys., 108, 179 (1986).

[28] W.S. Warren, D.P. Weitekamp, and A. Pines, J. Chem. Phys., 73, 2084 (1980).

[29] M. Munowitz, Coherence and NMR, (John Wilcy \& Sons Press, New York, 1988).

[30] R.R. Ernst and G. Bodenhausen, Principles of Magnetic Resonance in One and Two Dimensions, (Oxford University Press, Oxford, 1990).

[31] N.Q. Fan, B. Heaney, J. Clarke, D. Newitt, L.L. Wald, E.L. Hahn, A. Bielecki, and A. Pines, Proc. of IEEE Trans. Mag., 25 1989) 1193.

[32] C. Connor, J. Chang, A. Pines, J. Magn. Reson., 82, 387 (1989). 
[33] C. Connor, J. Chang, A. Pines, Rev. Sci. Instrum., in press.

[34] D. Suter and R.R. Ernst, Phys. Rev.B 32, 5608 (1985).

[35] P. Caravatti, J.A. Deli, G. Bodenhausen, and R.R. Ernst, J. Am. Chem. Soc., 104, 5506 (1982).

[36] N.M. Szevernyi, M.J. Sullivan, and G.E. Maciel, J. Magn. Reson., 47, 462 (1982).

[37] P.M. Henrichs and M. Linder, J. Magn. Reson., 58, 458 (1984).

[38] P. Caravatti, P. Neuenschwander, and R.R. Ernst, Macromolecules, 18, 119 (1985).

[39] D. Suter and R.R. Ernst, Phys. Rev., B 25, 6028 (1982).

[40] C.E. Bronniman, N.M. Szevernyi, and G.E. Maciel, J. Chem. Phys., 79, 3694 (1983).

[41] J. Jeener, B.H. Meier, P. Bachmann, and R.R. Ernst, J. Chem. Phys., 71, 4546 (1979).

[42] W.P. Aue, E. Bartholdi, and R.R. Ernst, J. Chem. Phys., 64, 2229 (1976).

[43] N.F. Ramsey and R.V. Pound, Phys. Rev., 81, 278 (1951).

[44] A.G. Anderson, Phys. Rev., 115, 863 (1959); 125, 1517 (1962).

[45] A.G. Redfield, Phys. Rev., 130, 589 (1963).

[46] R.L. Strombotne and E.L. Hahn, Phys. Rev.A 133, 1616 (1964).

[47] S. Vega, Adv. Magn. Reson., 6, 259 (1973).

[48] B.N. Provotorov, Zh. Eksp. Teor. Fiz., 41, 1582 (1961) [Sov. Phys.-JETP 14, 1126 (1962)].

[49] R. Tycko, J. Mag. Reson., 75, 193 (1987).

[50] R. Tycko, Phys. Rev. Lett., 60, 2734 (1988). 
[51] R. Tycko, J. Chem. Phys.92, 5778 (1990).

[52] E.L. Hahn, Phys. Rev. 80, 580 (1950).

[53] H.Y. Carr and E.M. Purcell, Phys. Rev., 94, 630 (1954).

[54] W.A. Anderson and J.T. Arnold, Phys. Rev., 94, 497 (1954).

[55] J.S. Waugh, L.M. Huber, U. Haeberlen Phys. Rev. Lett., 20, 180 (1968).

[56] E.R. Andrew, A. Bradbury, R.G. Eades, Nature, 182, 1659 (1958).

[57] E. M. Andrew, Arch. Sci., (Geneva) 12, 103(1959)

[58] T.J. Lowe, Phys. Rev. Lett., 2, 285 (1959).

[59] C.P. Slichter, Principles of Magnetic Resonance, (Springer-Verlag, Berlin, 1978).

[60] D.L. VanderHart, J. Chem. Phys.84, 1196 (1985).

[61] A. Messiah, Quantum Mechanics (North-Holland, Amsterdam, 1961).

[62] U. Haeberlen and J.S. Waugh, Phys. Rev.175, 453 (1968).

[63] L.L. Buishvili and M.G. Menabde, Sov. Phys.-JETP 50, 1176 (1979).

[64] N.N. Bogolubov and Y.A. Mitropolsky, Asymptotic methods in the theory of non-linear oscillations (Gordon and Breach, New York, 1961).

[65] Z. Peng, B.Q. Sun, and A. Pines, in preparation.

[66] S. Ganapathy, S. Schramm, and E. Oldfield, J. Chem. Phys., 77, 4360 (1982).

[67] P. Man, J. Magn. Reson., 67, 78 (1986); ibid, 77, 148 (1988).

[68] M. Hamermesh, Group Theory and Its Application To Physical Problems, Dover, New York.

[69] A.D. McLaren, Math. Comput., 17, 361 (1963).

[70] S.I. Konyaev, Math. Note, 25, 629 (1979). 
[71] A.E. Mefed, Soviet J. Exp. Theor. Phys., 86, 302 (1984).

[72] A. Samoson, E. Lippmaa and A. Pines, Mol. Phys., 65,1013 (1988).

[73] A. Llor and J. Virlet, Chem. Phys. Lett., 152, 248 (1988).

[74] A. Pines, at Ninth Experimental NMR Conference Bad Aussee, Austria (May 1988).

[75] G.C Chingas, C.J. Lee, E. Lippmaa, K.T. Mueller, A. Pines, A. Samoson, B.Q. Sun, D. Suter, and T. Terao, in Proc. XXIV Congress Ampère, Poznaǹ, Poland 1988 J. Stankowski, N. Pis̀lewski, and S. Idziak, Eds., D62.

[76] K.T. Mueller, B.-Q. Sun, G.C Chingas, J.W. Zwanziger, T. Terao, and A. Pines, J. Magn. Reson., 86, 470 (1990).

[77] C.J. Lee, NMR with Generalized Dynamics of Spin Spatial Coordinates PhD. Thesis (University of California, Berkeley, 1988).

[78] B.F. Chmelka, K.T. Mueller, A. Pines, J. Stebbins, Y. Wu and J.W. Zwanziger, Nature (Lodon), 339, 42 (1989).

[79] A. Samoson AND A. Pines, Rev. Sci. Instrum. 60, 3239 (1989).

[80] K.T. Mueller, Ph.D. Dynamic angle spinning and double rotation, $\mathrm{PhD}$. Thesis (University of California, Berkeley, 1991).

[81] K.T. Mueller, G. Chingus, and, A. Pines, Sci. Rev. Instrum., 62, 1445 (1991).

[82] K.T. Mueller, E.W. Wooten, and A. Pines, J. Magn. Reson., 92620 (1991).

[83] C. Ye, B.Q. Sun, and G.E. Maciel, J. Magn. Reson., 70, 241 (1986).

[84] S. Vega, J. Chem. Phys.68, 5518 (1978).

[85] S. Vega and A. Pines, J. Chem. Phys.66, 5624 (1977).

[86] A. Wokaun and R.R. Ernst, J. Chem. Phys.67, 1752 (1977).

[87] A. Bax, N.M. Szeverenyi, and G. Maciel, J. Magn. Reson., 52, 147 (1983). 
[88] T. Terao, H. Miura, and A. Saika, J. Chem. Phys.85, 3816 (1986).

[89] L. Frydman, Y. Lee, and A. Pines, in preparing.

[90] K.T. Mueller, Y. Wu, B.F. Chmelka, J. Stebbins, and A. Pines, J. Am. Chem. Soc.113, 32 (1991).

[91] F.D. Doty AND P.D. Ellis, Rev. Sci. Instrum. 52, 1868 (1981).

[92] R.J. Stephenson, "Mechanics and Properties of Matter," John Wiley and Sons, New York, 1969.

[93] J. Schaefer and E. O. Stejskal, J. Am. Chem. Soc. 98, 1031 (1976).

[94] F. Bloch, Phys. Rev., 94, 496 (1954).

[95] M.M. Maricq and J. S. Waugh, J. Chem. Phys., 70, 3300 (1979).

[96] J. Herzfeld and A. E. Berger, J. Chem. Phys., 73, 6012 (1980).

[97] N.J. Clayden, C.M. Dobson, L.Y. Lian, and D.J. Smith, J. Magn. Reson., 69, 476 (1986).

[98] W.H. Press, B.P. Flannery, S.A. Teukolsky, and W.T. Vetterling, Numerical Recipes. (Cambridge University Press, 1988).

[99] W.T. Dixon, J. Chem. Phys., 77, 1800 (1982).

[100] D.P. Raleigh, E.T. Olejniczak, S. Vega, and R.G. Griffin, J. Magn. Reson., 72, 238 (1986).

[101] E. Kundla, A. Samoson, and E. Lippmaa, Chern. Phys. Lett., 33. 229 (1981).

[102] A. Samoson, E. Kundla, and E. Lippmaa, J. Magn. Reson., 49, 350 (9182).

[103] S. Ganapathy, S. Schramm, and E. Oldfield, J. Chem. Phys., 77, 4360 (1982).

[104] Y, Wu, B.Q. Sun, A. Pines, A. Samoson, and E. Lippmaa, J. Magn. Reson.89, 296 (1990).

[105] B.-Q. Sun, Y. Wu, and A. Pines, to be published. 
[106] B.Q. Sun and A. Pines, in preparation.

[107] J.H Van Vleck, Phys. Rev.74, 1168 (1948).

[108] Z. Peng, B.Q. Sun, Y. Wu, and A. Pines, to be published.

[109] U. Haeberlen and J.S. Waugh, Phys. Rev., 175, 453 (1968).

[110] J.P. Amoureux and C. Fernandez, Magn. Reson. in Chem., 28, 5 (1990).

[111] D.W. Alderman, S.M. Solum, and D.M. Grant, J. Chem. Phys., 84, 3717 (1986).

[112] A. Samoson and E. Lippmaa, J. Magn. Reson. 84, 410 (1989).

[113] A. Samoson, B.Q. Sun, and A. Pines, to be published in Pulsed Magnetic Resonance: NMR, ESR, and Optics (a recognition of E. L. Hahn), D.M.S. Bagguley, Ed. (Clarendon, Oxford, 1992).

[114] J. S. Waugh, L. M. Huber, and U. Haeberlen, Phıs. Rev. Lett., 20, 180 (1968).

[115] U. Haeberlen, High Resolution NMR in Solid ve Averaging Adv. Magn. Reson., Suppl. 1 (Academic, New York, 1976).

[116] E.P. Wigner, Group Theory and Its Application to the Quantum Mechics of Atomic Spectra, (Academic, New York, 1959).

[117] A.R. Edmonds, Angular Momentum in Quantum Mechanics, (Princeton University, Princeton, 1974).

[118] B.L. Silver, Irreducible tensor Methods, (Academic, New York, 1962).

[119] M. Lee and W.I. Goldberg, Phys. Rev., A 140, 1261 (1965).

[120] A.C. Cheung, D.M. Rank, C.H. Townes, D.C. Thomton, W.J. Welch, Phys. Rev. Lett., 21, 1701 (1968).

[121] A.J. Alexander, H.W. Kroto, D.R. Walton, J. Mol. Spectrosc., 62, 175 (1976)

[122] L.W. Avery, N.W. Broten, J.M. Macleod, T. Oka, H.W. Kroto, Astrophys. J., 205, L173 (1976). 
[123] V.H. Hintenberger, J. Franzen, K,D. Schuy, Z. Naturforsch. Teil A, 18, 1236 (1963).

[124] H.W. Kroto, J.R. Heath, S.C. O'Brien, R.F. Curl, and R.E. Smalley, Nature 318, 162 (1985).

[125] H.W. Kroto, Science 242, 1139 (1988).

[126] E.A. Rohlfing, D.M. Cox, and A. Kaldor, J. Chem. Phys., 81, 3322 (1984).

[127] W. Kraetschmer, L.D. Lamb, K. Fostlropoulos, and D.R. Huffman, Nature, 347, 354 (1990).

[128] W.E. Pichett, Nature, 351, 602 (1991).

[129] P.W. Stephens, L. Mihaly, P.L. Lee, R.L. Whetten, S.-M Huang, R. Kaner, F. Deiderich, and K. Holczer, Nature, 351, 632 (1991).

[130] B.W.H. Van Beest, G.L. Kramer, and R.A. Van Santen, Phys. Rev. Lett., 64, 1955 (1990).

[131] G.J. Kramer, N.P. Farragher, B.W.H. Beest, and R.A. Santen, Phys. Rev., B 43, 5068 (1991).

[132] R.C. Haddon, A.F. Hebard, M.J. Rosseinsky, D.W. Murphy, S.J. Duclos, K.B. Lyons, B. Miller, J.M. Rosamilla, R.M. Fleming, A.R. Kortan, S.H. Glarum, A.V. Makhija, A.J. Muller, R.H. Eick, S.M. Zahurak, R. Tycko, G. Dabbagh, and F.A. Thiel, Nature, 350, 320 (1991).

[133] A.F. Hebard, M.J. Rosseinsky, R.C. Haddon, D.W. Murphy, S.H. Glarum, T.M. Pastra, A.P. Ramirez, and A.R. Kortan, Nature, 350, 600 (1991).

[134] R.D. Johnson, D.S. Bethune, J. Am. Chem. Soc., 112, 8983 (1990).

[135] R. Taylor, J.P. Hare, A.K. Abdul-Sada, and H.W. Kroto, J. Chem. Soc. Chem. Comm., 1423 (1990).

[136] D.S. Bethune, G. Meljer, W.C. Tong, and H.J. Rosen, Chem. Phys. Lett., 174, 219 (1990). 
[137] R. Tycko, R.C. Dabbagh, G. Dabbagh, S.H. Glarum, D.C. Douglass, and A.M. Mujsce, J. Phys. Chem., 95, 518 (1991).

[138] R. Tycko, G. Dabbagh, R.M. Fleming, R.C. Haddon, A.V. Makhija, and S.M. Zahurak, Phys. Rev. Lett., in press.

[139] C.S. Yannoni, R.D. Johnson, G. Meijer, D.S. Bethune, and J.R. Salem, J. Phys. Chem., 95, 9 (1991).

[140] C.S. Yannoni, D.S. Bethune, G. Meijer, and J.R. Salem, J. Am. Chem. Soc., 95, 9 (1991).

[141] P.A. Heiney, J.E. Fischer, A.R. McGhie, W.J. Romanow, A.M. Denenstein, J.P. McCauley, and A.B. Smith, Phys. Rev. Lett., in press.

[142] J.A. Pople, Mol. Phys., 1, 168 (1958).

[143] S. Alexander, A. Baram, and Z. Luz, Mol. Phys., 27, 441 (1974).

[144] S. Alexander, Z. Luz, Y. Naor, and R. Poupko, Mol. Phys., 33, 1119 (1977).

[145] D.E. Wemmer, Some Double Resonance and Multiple Quantum NMR Studies in Solids, PhD. Thesis (University of California, Berkeley, 1978).

[146] D.E. Wemmer, D.J. Ruben, and A. Pines, J. Am. Chem. Soc., 103, 28 (1981).

[147] R.J. Wittebort, M.G. Usha, D.J. Ruben, D.E. Wemmer, and A. Pines, J. Am. Chem. Soc., 110, 56 (1988).

[148] H.W. Spiess, Rotation of Molecules and Nuclear Spin Relaxation in NMR: Basic Principles and Progress., Vol. 15 (Berlin-HeidelBerg-New York: Springer 1978).

[149] H.W. Spiess, Chem. Phys., 6, 217 (1974).

[150] H.W. Speiss, Chem. Phys., 6, 226 (1974).

[151] H. Sillescu, Ber. Bunsenges. Phys. Chem., 75,283 (1971). 
[152] K. Hensen, W.O. Riede, H. Sillescu, A.V. Wittgenstein, J. Chem. Phys., 61, 4365 (1974).

[153] D.P. Burum and W.K. Rihm, J. Chem. Phys., 70, 3553 (1979).

[154] J.H. Freed, G.V. Bruno, and C.F. Polnaszek, J. Phys. Chem., 75, 3386 (1971).

[155] P. Rigny, Physica, 59, 707 (1972).

[156] J.S. Griffith, The Theory of Transition-Metal Ions, (Cambridge Univ. Press, 1961.)

[157] J.S. Griffith, Mol. Phys., 3, 79 (1960).

[158] J.S. Griffith, The irreducible Tensor Method for Molecular Symmetry Group (Prentice-Hill, 1962).

[159] J.S. Griffith, Trans. Faraday Soc., 56,193 (1960).

[160] G.F. Koster, J.O. Dimmock, R.G. Wheeler, and H. Statz, Properties of the thirty-Two point Group, (MIT press, 1963).

[161] R.M. Golding, Mol. Phys., 21, 157 (1971).

[162] R.G. Gordan and R.P. MaGinnis, J. Chem. Phys., 49, 2455 (1968). 

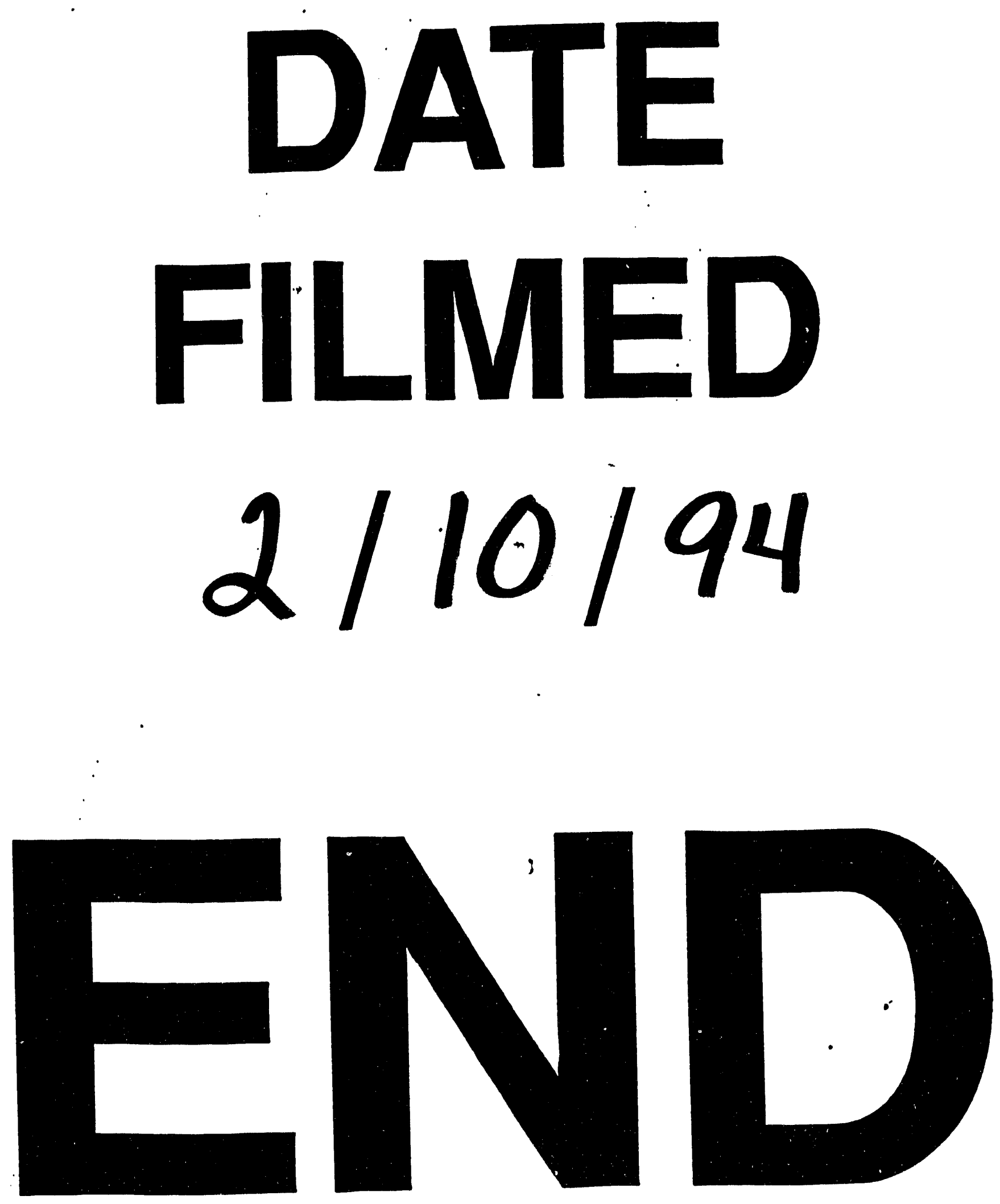
